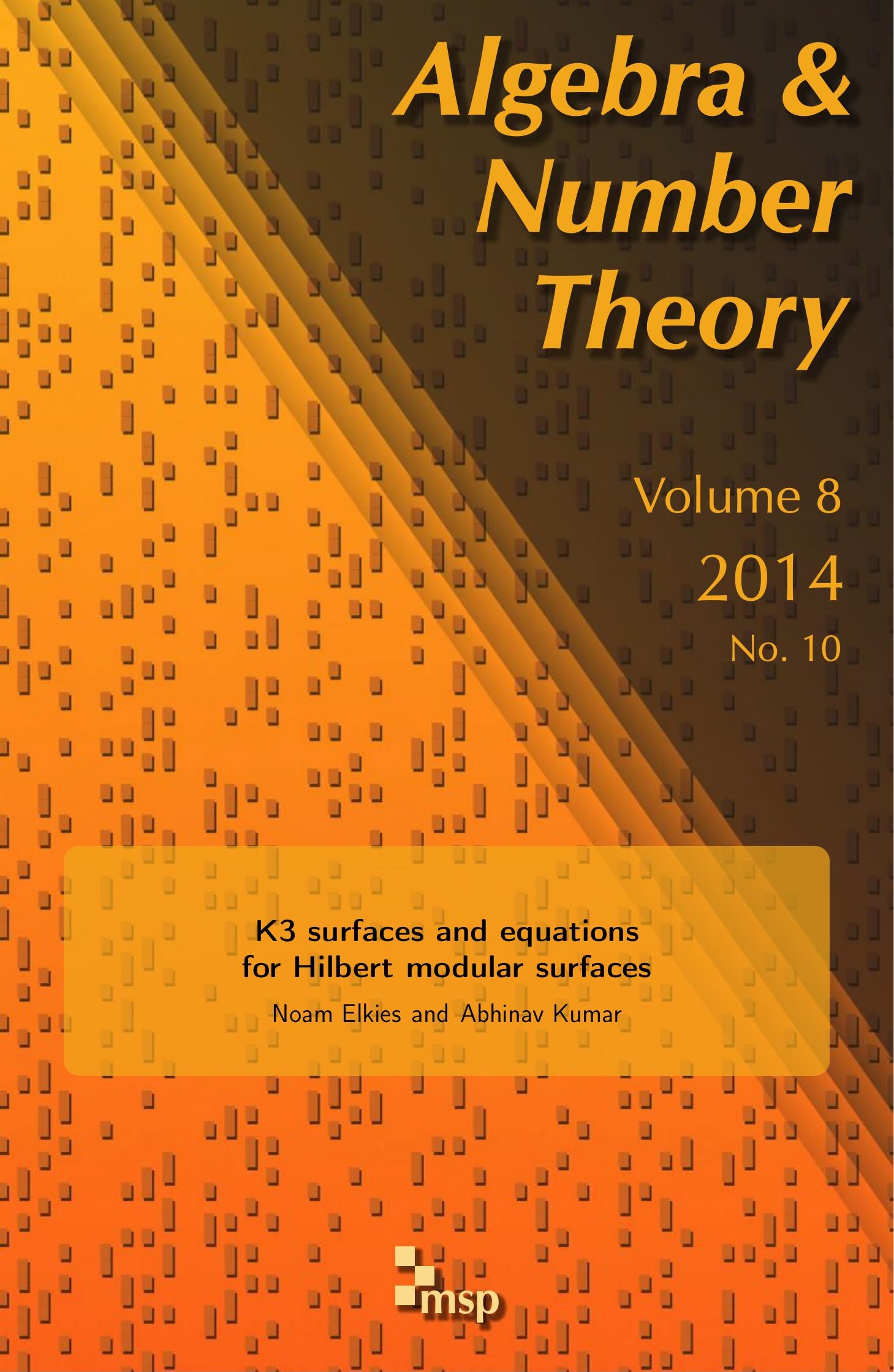




\title{
K3 surfaces and equations for Hilbert modular surfaces
}

\author{
Noam Elkies and Abhinav Kumar
}

\begin{abstract}
We outline a method to compute rational models for the Hilbert modular surfaces $Y_{-}(D)$, which are coarse moduli spaces for principally polarized abelian surfaces with real multiplication by the ring of integers in $\mathbb{Q}(\sqrt{D})$, via moduli spaces of elliptic K3 surfaces with a Shioda-Inose structure. In particular, we compute equations for all thirty fundamental discriminants $D$ with $1<D<100$, and analyze rational points and curves on these Hilbert modular surfaces, producing examples of genus- 2 curves over $\mathbb{Q}$ whose Jacobians have real multiplication over $\mathbb{Q}$.
\end{abstract}

\section{Introduction}

Hilbert modular surfaces have been objects of extensive investigation in complex and algebraic geometry, and more recently in number theory. Since Hilbert modular varieties are moduli spaces for abelian varieties with real multiplication by an order in a totally real field, they have intrinsic arithmetic content. Their geometry is enriched by the presence of modular subvarieties.

In [Hirzebruch 1973; Hirzebruch and van de Ven 1974; Hirzebruch and Zagier 1977] the geometric invariants of many of these surfaces were computed, and they were placed within the Enriques-Kodaira classification. A chief aim of the present work is to compute equations for birational models of some of these surfaces over the field of rational numbers.

More precisely, let $D$ be a positive fundamental discriminant, i.e., the discriminant of the ring of integers $\mathcal{O}_{D}$ of the real quadratic field $\mathbb{Q}(\sqrt{D})$. The quotient $\operatorname{PSL}_{2}\left(O_{D}\right) \backslash\left(\mathcal{H}^{+} \times \mathcal{H}^{-}\right)$(where $\mathcal{H}^{+}$and $\mathcal{H}^{-}$are the complex upper and lower half-planes) parametrizes abelian surfaces with an action of $\mathcal{O}_{D}$. It has a natural

Elkies was supported in part by NSF grants DMS-0501029 and DMS-1100511. Kumar was supported in part by NSF grants DMS-0757765 and DMS-0952486, and by a grant from the Solomon Buchsbaum Research Fund. The research was started when Kumar was a postdoctoral researcher at Microsoft Research. He also thanks Princeton University for its hospitality during Fall 2009.

MSC2010: primary 11F41; secondary 14G35, 14J28, $14 \mathrm{~J} 27$.

Keywords: elliptic K3 surfaces, moduli spaces, Hilbert modular surfaces, abelian surfaces, real multiplication, genus- 2 curves. 
compactification $Y_{-}(D)$, obtained by adding finitely many points and desingularizing these cusps.

These surfaces $Y_{-}(D)$ have models defined over $\mathbb{Q}$, and the main goal of this paper is to describe a method to compute explicit equations for these models, as well as to carry out this method for all fundamental discriminants $D$ with $1<D<100$. This felt like a good place to stop for now, though these calculations may be extended to some higher $D$, as well as to non-fundamental discriminants.

We briefly summarize the method, which we describe in more detail in later sections. The method relies on being able to explicitly parametrize K3 surfaces that are related by Shioda-Inose structure to abelian surfaces with real multiplication by some $\mathcal{O}_{D}$. The K3 surface corresponding to such an abelian surface has NéronSeveri lattice containing $L_{D}$, a specific indefinite lattice of signature $(1,17)$ and discriminant $-D$. In all our examples, we obtain the moduli space $\mathcal{M}_{D}$ of $L_{D^{-}}$ polarized K3 surfaces as a family of elliptic surfaces with a specific configuration of reducible fibers and sections.

We then use the 2- and 3-neighbor method to transform to another elliptic fibration, with two reducible fibers of types $\mathrm{II}^{*}$ and $\mathrm{III}^{*}$ respectively. This lets us read off the map (generically one-to-one) of moduli spaces from $\mathcal{M}_{D}$ into the 3-dimensional moduli space $\mathcal{A}_{2}$ of principally polarized abelian surfaces, using the formulae from [Kumar 2008]. The image of $\mathcal{M}_{D}$ is the Humbert surface corresponding to discriminant $D$. The Hilbert modular surface $Y_{-}(D)$ itself is a double cover of the Humbert surface, branched along a union of modular curves. We use simple lattice arguments to obtain the branch locus, and pin down the exact twist for the double cover by counting points on reductions of the related abelian surfaces modulo several primes. In all our examples, the Humbert surface happens to be a rational surface (i.e., birational to $\mathbb{P}^{2}$ over $\mathbb{Q}$ ), and we display the equation of $Y_{-}(D)$ as a double cover of $\mathbb{P}^{2}$ branched over a curve of small degree. We analyze these equations in some detail, attempting to produce rational or elliptic curves on them, with the intent of producing several (possibly infinitely many) examples of genus- 2 curves whose Jacobians have real multiplication. When $Y_{-}(D)$ is a K3 surface, it often has very high Picard number (19 or 20), and we attempt to compute generators for the Picard group. When $Y_{-}(D)$ is an honestly elliptic surface, we analyze the singular fibers and the Mordell-Weil group, and attempt to compute a basis for the sections.

To our knowledge, this is the first algebraic description of most of these surfaces by explicit equations. We outline some related work in the literature. Wilson [1998; 2000] obtained equations for the Hilbert modular surface $Y_{-}(5)$ corresponding to the smallest fundamental discriminant $D>1$. Van der Geer [1988] gives a few examples of algebraic equations for Hilbert modular surfaces corresponding to a congruence subgroup of the full modular group (in other words, abelian surfaces 
with some level structure). Humbert surfaces have also been well-studied in the literature, and Runge [1999] and Gruenewald [2008] have obtained equations for some of these. However, these equations are quite complicated, and do not shed as much light on the geometry of Hilbert modular surfaces. While the methods are simpler, involving theta functions and $q$-expansions, the result is analogous to exhibiting the modular polynomial whose zero locus in $\mathbb{A}^{1} \times \mathbb{A}^{1}$ is a singular model of the complement of the cusps in the modular curve $X_{0}(N)$. The coefficients of these polynomials can quickly become enormous. We believe that our approach, giving simpler equations for these surfaces together with their maps to $\mathcal{A}_{2}$, is more conducive to an investigation of arithmetic properties.

It is our hope that these equations will be of much help in subsequent arithmetic investigation of these surfaces. For instance, they should provide a testing ground for many conjectures in Diophantine geometry, because of the abundance of rational curves and points. Another direction of future investigation is to use these equations to investigate modularity of the corresponding abelian surfaces. Modularity of abelian varieties with real multiplication over $\mathbb{Q}$ is now proven, by combining results of Ribet [2004] with the recent proof of Serre's conjecture by Khare and Wintenberger [2009a; 2009b]. However, unlike the case of dimension 1, where one has modular parametrizations and very good control of the moduli spaces, the situation in dimensions 2 and above is much less clear. For instance, it is not at all clear how to find a modular form corresponding to a given abelian surface with real multiplication. ${ }^{1}$ We hope that the abundance of examples provided by these equations will help pave the path for a better understanding of the 2-dimensional case. For example, in [Dembélé and Kumar 2013], our formulas are combined with efficient computation of Hilbert modular forms to find examples of simple abelian surfaces over real quadratic fields, with everywhere good reduction. An example of such an abelian surface is the Jacobian of the genus- 2 curve

$$
\begin{aligned}
2 y^{2} & =x^{6}-\tau x^{5}+74 x^{4}-14 \tau x^{3}+267 x^{2}-13 \tau x+46 \\
& =\left(x^{3}-\frac{1+\tau}{2} x^{2}+13 x+\frac{3-\tau}{2}\right)\left(x^{3}+\frac{1-\tau}{2} x^{2}+13 x-\frac{3+\tau}{2}\right),
\end{aligned}
$$

where $\tau=\sqrt{193}$ (the curve and its Jacobian can in fact be defined over $\mathbb{Q}$, but the Jacobian attains everywhere good reduction and real multiplication by $\mathcal{O}_{17}$ only over $\mathbb{Q}(\tau)$ ).

${ }^{1}$ Suppose $A / \mathbb{Q}$ is an abelian surface with $\mathbb{Q}$-endomorphisms by $\mathcal{O}_{D}$, and let $\varphi=\sum_{n} a_{n} q^{n}$ be an eigenform with every $a_{n} \in O_{D}$. If $\varphi$ corresponds to $A$ then counting points over $\mathbb{F}_{p}$ and $\mathbb{F}_{p}^{2}$ determines each $a_{p}$ up to Galois conjugation. But conceivably there might be some eigenform $\varphi^{\prime}=\sum_{n} a_{n}^{\prime} q^{n}$, different from both $\varphi$ and its Galois conjugate, such that each $a_{p}^{\prime}$ equals either $a_{p}$ or the Galois conjugate of $a_{p}$; if that happens, we do not know how to decide which eigenform corresponds to $A$. Likewise for abelian varieties of dimension 3 and higher. 
The outline for the rest of the paper is as follows. In Section 2, we describe the relevant background on K3 surfaces and their moduli spaces, and their connection to moduli spaces of abelian surfaces via Shioda-Inose structures. In Section 3, we describe the Hilbert modular surfaces and the corresponding moduli spaces of K3 surfaces. In Section 4, we precisely describe our methods to compute their equations. Section 5 describes the 2- and 3-neighbor method in more detail. The rest of the paper consists of detailed examples of Hilbert modular surfaces for the discriminants less than 100 , as well as an arithmetic investigation of these surfaces.

An accompanying online supplement contain formulas for the Igusa-Clebsch invariants, as well as a description of the parametrizations exhibited for the moduli spaces of K3 surfaces in the paper, and the details of the neighbor steps to transform to a fibration with II* and III* fibers. The online supplement can be obtained from this article's publication page. The files are also available as part of the source package for the arXiv version of this article (arXiv 1209.3527). See the file README.txt for an overview.

\section{Moduli spaces of abelian surfaces and lattice polarized K3 surfaces}

Throughout this section, we work with K3 surfaces over a field $k$ of characteristic 0 . When convenient, we will suppose $k \subseteq \mathbb{C}$, and use transcendental methods.

2.1. K3 surfaces. A $K 3$ surface $X$ over $k$ is a projective algebraic nonsingular surface with $h^{1}\left(X, \mathcal{O}_{X}\right)=0$ and $K_{X} \cong \mathcal{O}_{X}$. For such a surface, $H^{2}(X, \mathbb{Z})$ is torsionfree, and when endowed with the cup-product form becomes a 22-dimensional lattice, abstractly isomorphic with $\Lambda:=E_{8}(-1)^{2} \oplus U^{3}$. Here $E_{8}$ is the even unimodular lattice in eight dimensions, $U$ is the hyperbolic plane with Gram matrix $\left(\begin{array}{ll}0 & 1 \\ 1 & 0\end{array}\right)$, and for any lattice $\Lambda$ and real number $\alpha$, the lattice $\Lambda(\alpha)$ consists of the same underlying abelian group with the form multiplied by $\alpha$. The Néron-Severi group NS $(X)$ of algebraic divisors defined over $\bar{k}$ modulo algebraic equivalence, which for a K3 surface is the same as linear or numerical equivalence, is a primitive sublattice of $\Lambda$ of signature $(1, \rho-1)$, where $\rho \in\{1, \ldots, 20\}$ is the Picard number of $X$. The orthogonal complement of $\operatorname{NS}(X)$ is the transcendental lattice $T_{X}$. There is a Torelli theorem for K3 surfaces, due to Piatetski-Shapiro and Shafarevich [1971] and Friedman [1984], which describes the moduli space of K3 surfaces with a fixed polarization. More generally, let $L$ be an even nondegenerate lattice of signature $(1, r-1)$, with $r \in\{1, \ldots, 20\}$. Assume that $L$ has a unique primitive embedding in $\Lambda$, up to isometries of $\Lambda$. Then there is a coarse moduli space $\mathcal{F}_{L}$ of $L$-polarized $\mathrm{K} 3$ surfaces $(X, j)$, where $j: L \rightarrow \mathrm{NS}(X)$ is a primitive lattice embedding such that $j(L)$ contains a pseudo-ample class on $X$. The space $\mathcal{F}_{L}$ is isomorphic to the quotient of an appropriate fundamental 
domain

$$
\Omega_{L}=\mathbb{P}\left(\left\{\omega \in L^{\perp} \otimes \mathbb{C} \mid\langle\omega, \omega\rangle=0,\langle\omega, \bar{\omega}\rangle>0\right\}\right)
$$

by an arithmetic subgroup $\Gamma_{L}$, which is the image of

$$
\Gamma(L)=\{\sigma \in O(\Lambda) \mid \forall x \in L, \sigma(x)=x\}
$$

in $O\left(\Lambda^{\perp}\right)$. (Here, we have fixed an embedding $i: L \rightarrow \Lambda$, so we may use its orthogonal complement $L^{\perp}$.) Therefore $\mathcal{F}_{L}$ is a quasiprojective variety.

In fact, there is a fine moduli space $\mathcal{K}_{L}$ of marked pseudo-ample $L$-polarized $\mathrm{K} 3$ surfaces, i.e., $(X, \phi)$, where $\phi: H^{2}(X, \mathbb{Z}) \rightarrow \Lambda$ is an isomorphism (a marking) such that $\phi^{-1}(L) \subseteq \mathrm{NS}(X)$. There is a period map which associates to such a marked K3 surface the class of the global algebraic 2-form up to scaling, giving a point $[\omega] \in \mathbb{P}\left(L^{\perp} \otimes \mathbb{C}\right)$. Furthermore, $\omega \cup \omega=0$ and $\omega \cup \bar{\omega}>0$. This domain $\Omega_{L}$ consists of two copies of a bounded Hermitian domain of type $\mathrm{IV}_{20-r}$. The period map $(X, \phi) \rightarrow\left[\omega_{X}\right]$ sets up an isomorphism between the moduli space $\mathcal{K}_{L}$ and the period domain $\Omega_{L}$, using the Torelli theorem and the surjectivity of the period map [Kulikov 1977; Persson and Pinkham 1981]. The quotient $\Gamma_{L} \backslash \mathcal{K}_{L} \cong \Gamma_{L} \backslash \Omega_{L}$ forgets the marking, and describes a coarse moduli space of $L$-polarized K3 surfaces. For details, the reader may consult [Nikulin 1979a; Dolgachev 1996].

2.2. Elliptic K3 surfaces. We shall be especially interested in moduli spaces of elliptic K3 surfaces. In this paper, an elliptic K3 surface will be a K3 surface $X$ with a relatively minimal genus-1 fibration $\pi: X \rightarrow \mathbb{P}^{1}$, together with a section. In other words, we may write a Weierstrass equation of $X$ over $\mathbb{P}_{t}^{1}$ as

$$
y^{2}+a_{1}(t) x y+a_{3}(t) y=x^{3}+a_{2}(t) x^{2}+a_{4}(t) x+a_{6}(t),
$$

with $a_{i}(t)$ a polynomial in $t$ of degree at most $2 i$. (More canonically, each $a_{i}$ is a homogeneous polynomial of degree $2 i$ in the two homogeneous coordinates of $\mathbb{P}_{t}^{1}$.) Of course, this Weierstrass equation describes the generic fiber of $X$; to understand the reducible special fibers, one can use Tate's algorithm [1975] to blow up the singular points and describe the minimal proper model. (This will also detect when a Weierstrass equation (1) is equivalent to one with each $a_{i}$ vanishing to order at least $i$ at some $t_{0}$, that is, when the equation gives not a K3 elliptic surface but a rational or constant one.) The singular fibers are classified by Kodaira and Néron, and the non-identity components of any reducible fiber $\pi^{-1}(v)$ contribute an irreducible root lattice (scaled by -1 ), say $L_{v}$, to the Néron-Severi lattice. The trivial lattice is defined to be

$$
T=\mathbb{Z} O \oplus \mathbb{Z} F \oplus\left(\bigoplus_{v} L_{v}\right)
$$

(note that $O$ and $F$ span a copy of the hyperbolic plane $U$ ). 
A theorem of Shioda and Tate [Shioda 1972; 1990; Tate 1966b] shows that as long as the elliptic fibration is nontrivial (equivalently, it has at least one singular fiber), the Mordell-Weil group of $X$ over $\mathbb{P}^{1}$ is isomorphic to $\mathrm{NS}(X) / T$. In particular, we have the Shioda-Tate formula

$$
\rho(X)=2+\operatorname{rank} \operatorname{MW}\left(X / \mathbb{P}^{1}\right)+\sum_{v} \operatorname{rank} L_{v} .
$$

One may also compute the discriminant of the Néron-Severi lattice:

$$
|\operatorname{disc}(\mathrm{NS}(X))|=\frac{\operatorname{det}\left(H\left(X / \mathbb{P}^{1}\right)\right) \cdot \prod_{v} \operatorname{disc}\left(L_{v}\right)}{\left|\mathrm{MW}\left(X / \mathbb{P}^{1}\right)_{\mathrm{tors}}\right|^{2}},
$$

where $H\left(X / \mathbb{P}^{1}\right)$ is the height pairing matrix for a basis of the torsion-free part of the Mordell-Weil group of $X$ over $\mathbb{P}^{1}$.

If $F$ is the class of the fiber for an elliptic K3 surface $X$, then $F$ is primitive and nef, with $F^{2}=0$. Conversely, suppose that $F \in \mathrm{NS}(X)$ is a nonzero divisor class which is primitive and nef with $F^{2}=0$. Then a simple application of the Riemann-Roch theorem shows that $F$ or $-F$ must be effective, and since $F$ is nef, it must be represented by an effective divisor. Then a theorem of Piatetski-Shapiro and Shafarevich [1971, p. 559] shows that $F$ defines a genus-1 fibration.

Lemma 1. Let $F=\sum a_{i} E_{i}$ be a positive linear combination of smooth rational curves on a $\mathrm{K} 3$ surface $X$ such that $F \cdot E_{i}=0$ for all $i$, and such that $F$ is a primitive class in $\mathrm{NS}(X)$. Then $F$ defines a genus-1 fibration.

Proof. We have $F^{2}=\sum a_{i}\left(F \cdot E_{i}\right)=0$. By the above discussion, it is enough to show that $F$ is nef. Let $E^{\prime}$ be an irreducible curve on $X$. If $E^{\prime}$ is distinct from the $E_{i}$, then $E_{i} \cdot E^{\prime} \geq 0$ for every $i$, and so $F \cdot E^{\prime} \geq 0$. On the other hand, if $E^{\prime}=E_{i}$, say, then $F \cdot E^{\prime}=F \cdot E_{i}=0$. Therefore $F$ is nef.

Let us define an elliptic divisor to be a divisor satisfying the conditions of Lemma 1. We will frequently use this lemma, displaying an elliptic divisor by finding a subdiagram of the set of roots of $\mathrm{NS}(X)$, represented by smooth rational curves, which is an extended Dynkin diagram for a root lattice. Then the class of the appropriate linear combination of roots $F$ will define a genus-1 fibration. We need to know when such a fibration has a section.

Lemma 2. Suppose $F$ is an elliptic divisor defining a genus-1 fibration $\pi: X \rightarrow \mathbb{P}^{1}$. Suppose $D \in \mathrm{NS}(X)$ satisfies $D \cdot F=1$. Then $\pi$ has a section.

Proof. Consider the divisor $D^{\prime}=D+m F$, for some large integer $m$. Then $\left(D^{\prime}\right)^{2}=D^{2}+2 m$, while $K \equiv 0$, so the Riemann-Roch theorem implies

$$
h^{0}\left(D^{\prime}\right)-h^{1}\left(D^{\prime}\right)+h^{2}\left(D^{\prime}\right)=\frac{\left(D^{\prime}\right)^{2}}{2}+\chi\left(\mathcal{O}_{X}\right)=D^{2}+m+2 .
$$


Also, $h^{2}\left(D^{\prime}\right)=h^{0}\left(K-D^{\prime}\right)=h^{0}\left(-D^{\prime}\right)=0$ by Serre duality, and since $\left(-D^{\prime}\right) \cdot H=$ $-D \cdot H-m F \cdot H<0$ for any ample divisor $H$, as long as $m$ is large enough. Therefore we see that for large $m$, the divisor class $D^{\prime}$ can be represented by an effective divisor, which we may call $D^{\prime}$, by abuse of notation. Note that we still have $D^{\prime} \cdot F=1$. Decompose $D^{\prime}$ as $D_{\text {vert }}^{\prime}+D_{\text {hor }}^{\prime}$, where the first term contains all the components which lie along fibers of the genus- 1 fibration defined by $F$, and the second contains the other components. Then $D_{\text {hor }}^{\prime} \cdot F=1$. Therefore, $D_{\text {hor }}^{\prime}$ must be reduced and irreducible, and thus defines a section of the genus-1 fibration.

Corollary 3. Let $F$ be an elliptic divisor, and let $D_{1}$ and $D_{2}$ be two divisor classes such that $D_{1} \cdot F$ and $D_{2} \cdot F$ are coprime. Then the fibration has a section.

Proof. There exist integers $a_{1}, a_{2}$ such that $\left(a_{1} D_{1}+a_{2} D_{2}\right) \cdot F=1$. Now take $D=a_{1} D_{1}+a_{2} D_{2}$ in Lemma 1 .

Finally, we note a lattice-theoretic result which allows us to deduce that in all of the cases studied in this paper, the genus-1 fibration defined by an elliptic divisor $F$ has a section.

Proposition 4. Let $D$ be a fundamental discriminant, and let $L=U \oplus N(-1)$, where $U$ is the hyperbolic plane and $N$ a positive definite lattice of rank 16 and discriminant $D$. Suppose in addition that $N$ contains a sublattice isomorphic to $E_{8} \oplus E_{7}$. If $v \in L$ is a primitive vector with $v \cdot v=0$, then there exists $w \in L$ such that $v \cdot w=1$.

Proof. Suppose not. Then $\{v \cdot w: w \in L\}=c \mathbb{Z}$ for some integer $c>1$. Since $v$ is primitive, we can take a basis $v_{1}=v, \ldots, v_{16}$ of $L$. Then

$$
L^{\prime}=\mathbb{Z}(v / c)+\mathbb{Z} v_{2}+\cdots+\mathbb{Z} v_{16}
$$

is an integral lattice containing $L$ with index $c>1$. Since $L^{\prime} \supset L \supset U$, we have $L^{\prime}=U \oplus N^{\prime}(-1)$ (since $U$ is unimodular), with $N^{\prime}$ a positive definite lattice containing $N$ with index $c$. Then $N^{\prime}$ must be generated by $E_{8} \oplus E_{7}$ and a vector $x$ whose projection $x^{\perp}$ to the orthogonal complement of $E_{8} \oplus E_{7}$ has norm $D /\left(2 c^{2}\right)$. The dual lattice of $E_{8} \oplus E_{7}$ has norms congruent to 0 or $\frac{3}{2}$ modulo 2, so $x^{\perp}$ has norm 0 or $\frac{1}{2} \bmod 2$. Therefore $D / c^{2}$ must be an integer congruent to 0 or 1 modulo 4 . Since $D$ is a fundamental discriminant, this is impossible.

For the examples in this paper, we will often draw a Dynkin-type diagram indicating some of the roots of $\mathrm{NS}(X)$ for an elliptic $\mathrm{K} 3$ surface $X$, which will always be nodal classes (i.e., classes of smooth rational curves on $X$ ). We will outline an elliptic divisor $F$ by drawing a subdiagram in bold which cuts out an extended Dynkin diagram of an irreducible root lattice (the multiplicities will be omitted). Where convenient, we will also indicate the class of a divisor $D$ such that $D \cdot F=1$ (in some cases, there is an obvious node in the Dynkin diagram satisfying 
this property), or the classes of two divisors $D_{1}$ and $D_{2}$ such that $D_{1} \cdot F$ and $D_{2} \cdot F$ are coprime. This is not strictly necessary, because Proposition 4 guarantees the existence of such a divisor $D$, but having an explicit divisor might be useful for further calculations. Then $F$ defines another elliptic fibration with section on $X$, and we may proceed as in Section 5 to write down its Weierstrass equation.

\subsection{Kummer surfaces, Nikulin involutions and Shioda-Inose structures. Let $A$} be an abelian surface, and consider the involution $\iota$ on $A$ defined by multiplication by -1 in the group law. The quotient of $A$ by the group $\{1, \iota\}$ is a surface $Y^{\prime}$ with sixteen singularities, the images of the 2-torsion points of $A$. In fact, $Y^{\prime}$ may be realized as a quartic surface in $\mathbb{P}^{3}$ with sixteen ordinary double points (which is the maximum number of singularities possible for a quartic surface in $\mathbb{P}^{3}$ [Hudson 1990, p. 15]), by considering the linear system on $A$ corresponding to twice the theta divisor. The corresponding map is two-to-one from $A$ to $Y^{\prime}$.

Taking the minimal desingularization of $Y^{\prime}$ gives a $\mathrm{K} 3$ surface $Y$, the Kummer surface of $A$, which contains sixteen disjoint nodal classes coming from the blowups of the singular points. Note that if $A$ is defined over some number field $k$, then so is $Y=\operatorname{Km}(A)$. The Néron-Severi lattice of the surface $Y$ contains the saturation of the lattice spanned by the sixteen special nodal classes; this is a lattice $\Lambda_{\mathrm{Km}}$ of signature $(0,16)$ and discriminant $2^{6}$. Conversely, Nikulin showed that a K3 surface whose Néron-Severi lattice contains $\Lambda_{\mathrm{Km}}$ must be the Kummer surface of some complex torus. Of course, since $A$ is an abelian variety, $Y$ is a projective surface, so NS $(Y)$ contains an ample divisor as well.

We will be especially concerned with the case when $A=J(C)$ is the Jacobian of a curve of genus 2. Let $x_{0}, \ldots, x_{5}$ be the Weierstrass points of $C$. The embedding $\eta_{0}: C \rightarrow A$ given by $x \mapsto[x]-\left[x_{0}\right]$ gives a particular theta divisor on $A$, and the translates $\eta_{0}(C)+\left[x_{i}\right]-\left[x_{j}\right]$ with $0 \leq i<j \leq 5$ give fifteen more special divisors. The images of these sixteen divisors (tropes) on the Kummer surface of $A$ are disjoint rational curves, and each intersects six rational curves coming from the blowups of the singular points (i.e., the nodes). This classical configuration of tropes and nodes on the Kummer surface is called the $(16,6)$ configuration, and the intersection pairing describes a vertex- and edge-transitive bipartite graph of degree 6 on 32 vertices, isomorphic with the quotient of the 6-cube by central reflection.

Next, consider a K3 surface $X$ with a symplectic involution $\iota$, i.e., an involution $\iota$ that multiplies the algebraic 2-forms on $X$ by +1 (such an involution of $X$ is also known as a Nikulin involution). Then $\iota$ has eight fixed points on $X$, and the minimal desingularization $Y$ of the quotient $X /\{1, \iota\}$ is again a $\mathrm{K} 3$ surface. If in addition $Y$ is a Kummer surface $\mathrm{Km}(A)$ and the quotient map $\pi: X \rightarrow Y$ induces a Hodge isometry $\pi_{*}: T_{X}(2) \cong T_{Y}$, we say that $X$ and $A$ are related by a Shioda-Inose 
structure. We have a diagram

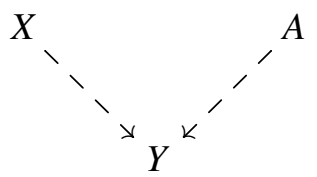

of rational maps of degree 2 , and Hodge isometries $T_{X}(2) \cong T_{Y} \cong T_{A}(2)$, thus inducing a Hodge isometry $T_{X} \cong T_{A}$. (Note: A Hodge isometry is an isometry of cohomology lattices compatible with the Hodge decomposition.)

Conversely, a theorem of Morrison [1984] shows that any Hodge isometry between $T_{X}$ and $T_{A}$ for a $\mathrm{K} 3$ surface $X$ and an abelian surface $A$ is induced by a Shioda-Inose structure.

\subsection{Elliptic K3 surfaces with $\mathrm{II}^{*}$ and $\mathrm{III}^{*}$ fibers, and curves of genus 2 . We shall} exploit such a Shioda-Inose correspondence between Jacobians of genus-2 curves and elliptic K3 surfaces with singular fibers of type II* and III*; equivalently, whose root lattices $L_{v}$ are $E_{8}$ and $E_{7}$ respectively. Let $C$ be a curve of genus 2 over $k$, and let

$$
y^{2}=f(x)=f_{6} x^{6}+\cdots+f_{0}=f_{6} \prod\left(x-\alpha_{i}\right)
$$

be a Weierstrass equation for $C$, with $f_{i} \in k$ and $\alpha_{i} \in \bar{k}$ (though in general $\alpha_{i} \notin k$ ). There exist polynomial functions $I_{2}(f), I_{4}(f), I_{6}(f)$ and $I_{10}(f)=\operatorname{disc}(f)$ of degrees $2,4,6,10$ in the coefficients of $f$ (the Igusa-Clebsch invariants of $f$ ), giving a well-defined point $\left(I_{2}: I_{4}: I_{6}: I_{10}\right)$ in weighted projective space $\mathbb{P}_{1,2,3,5}^{3}$ which does not depend on the choice of Weierstrass equation. The complement of the hyperplane $z_{4}=0$ (where $z_{4}$ is the last coordinate on $\mathbb{P}_{1,2,3,5}^{3}$ ) yields a coarse moduli space $\mathcal{M}_{2}$ of curves of genus 2 [Igusa 1960]. Note that $I_{10}$ is the discriminant of the sextic polynomial $f$, and therefore it cannot vanish. Also, the space $\mathcal{M}_{2}$ has a singular point at $(0: 0: 0: 1)$, corresponding to the curve $y^{2}=x^{5}+1$. Given $\alpha_{1}, \alpha_{2}, \alpha_{3}, \alpha_{5} \in k$, with $\alpha_{5} \neq 0$, it is not necessarily the case that one can construct a genus- 2 curve over $k$ with invariants $I_{d}=\alpha_{2 d}$. There is an obstruction in $\mathrm{Br}_{2}(k)$ : when it vanishes, the construction of $C$ is made explicit by Mestre [1991]. In any case, $C$ may always be defined over a quadratic extension of $k$. When $k$ is a finite field, the Brauer obstruction vanishes, and we may define $C$ over $k$. Also note that such a curve $C$ is unique only up to $\bar{k}$-isomorphism, since $\mathcal{M}_{2}$ is only a coarse moduli space.

The main result of [Kumar 2008] is the following.

Theorem 5. The elliptic $\mathrm{K} 3$ surface given by the Weierstrass equation

$$
y^{2}=x^{3}-t^{3}\left(\frac{I_{4}}{12} t+1\right) x+t^{5}\left(\frac{I_{10}}{4} t^{2}+\frac{I_{2} I_{4}-3 I_{6}}{108} t+\frac{I_{2}}{24}\right),
$$


which has elliptic fibers of type $E_{8}$ and $E_{7}$ respectively at $t=\infty$ and $t=0$, is related by a Shioda-Inose structure to the Jacobian of the genus-2 curve $C$ whose Igusa-Clebsch invariants are $\left(I_{2}: I_{4}: I_{6}: I_{10}\right)$.

Let $L$ be $U \oplus E_{8}(-1) \oplus E_{7}(-1)$. Then the above theorem gives an isomorphism

$$
\psi: \mathcal{M}_{2} \rightarrow \mathcal{E}_{E_{8}, E_{7}}
$$

between the coarse moduli space $\mathcal{M}_{2}$ of genus- 2 curves and the moduli space of elliptic K3 surfaces with an $E_{8}$ fiber at $\infty$ and an $E_{7}$ fiber at 0 . Furthermore, this correspondence is Galois-invariant: the Igusa-Clebsch invariants of $C$ and the Weierstrass coefficients of the K3 surface are defined over the same field. This is the key fact which leads to number-theoretic applications, such as computation of models of Shimura curves over $\mathbb{Q}$ in [Elkies 2008] or of Hilbert modular surfaces in this paper. However, note that $C$ may not be itself defined over the ground field, even though its Igusa-Clebsch invariants are.

Now, let $\mathcal{A}_{2}$ be the moduli space of principally polarized abelian surfaces. Note that the space $\mathcal{M}_{2}$ is the complement of the divisor in $\mathcal{A}_{2}$ consisting of points corresponding to the product of two elliptic curves. On the other hand, the moduli space $\mathcal{E}_{E_{8}, E_{7}}$ is an open subset of the moduli space $\mathcal{F}_{L}$ of K3 surfaces polarized by $L=U \oplus E_{8}(-1) \oplus E_{7}(-1)$. We may write such a $\mathrm{K} 3$ surface in Weierstrass form

$$
y^{2}=x^{3}+t^{3}\left(a t+a^{\prime}\right) x+t^{5}\left(b^{\prime \prime} t^{2}+b t+b^{\prime}\right) .
$$

It has an $E_{8}$ fiber at $t=\infty$ and at least an $E_{7}$ fiber at $t=0$. The discriminant of the cubic polynomial is

$$
\begin{aligned}
d=-t^{9}\left(27 b^{\prime \prime 2} t^{5}+54 b b^{\prime \prime} t^{4}+\right. & \left(4 a^{3}+27 b^{2}+54 b^{\prime} b^{\prime \prime}\right) t^{3} \\
& \left.+\left(12 a^{2} a^{\prime}+54 b b^{\prime}\right) t^{2}+\left(12 a a^{\prime 2}+27 b^{\prime 2}\right) t+4 a^{\prime 3}\right) .
\end{aligned}
$$

By Tate's algorithm, $b^{\prime \prime} \neq 0$ (otherwise we would have a rational elliptic surface), and the fiber at $t=\infty$ must be of type $E_{8}$, while the fiber at $t=0$ is of type $E_{7}$ if and only if $a^{\prime} \neq 0$, in which case one may set $a^{\prime}=-1$ by scaling $(x, y, t)$ appropriately. If on the other hand $a^{\prime}=0$, then the $E_{7}$ fiber gets promoted to an $E_{8}$ fiber (and no further, since $b^{\prime}$ cannot vanish, else we would have a rational elliptic surface). Therefore, the moduli space $\mathcal{E}_{E_{8}, E_{7}}$ is the complement in $\mathcal{F}_{L}$ of the hypersurface $a^{\prime}=0$ that corresponds to polarization by $U \oplus E_{8}(-1) \oplus E_{8}(-1)$.

When we do have $a^{\prime}=0$, it was shown by Inose that the K3 surface

$$
y^{2}=x^{3}+a t^{4} x+t^{5}\left(b^{\prime \prime} t^{2}+b t+b^{\prime}\right)
$$

has a Shioda-Inose structure, making it 2-isogenous with a product of two elliptic curves [Inose 1978; Shioda 2006; Kuwata and Shioda 2008; Clingher and Doran 2007; Elkies $\geq 2015]$. Recall that $b^{\prime} b^{\prime \prime} \neq 0$, and it follows from the formulas in 
[Inose 1978; Shioda 2006; Kumar 2008] that the $j$-invariants $j_{1}, j_{2}$ of the two elliptic curves are determined by

$$
\frac{j_{1}}{1728} \frac{j_{2}}{1728}=\frac{-a^{3}}{27 b^{\prime} b^{\prime \prime}}, \quad\left(1-\frac{j_{1}}{1728}\right)\left(1-\frac{j_{2}}{1728}\right)=\frac{b^{2}}{4 b^{\prime} b^{\prime \prime}} .
$$

Note that again the map is Galois invariant and invertible, since $a$ is only defined up to a cube root of unity (one may scale $x$ by a cube root of unity), and $b$ is only defined up to sign (one may scale $t$ by -1 ).

Putting everything together, we have the following proposition.

Proposition 6. There is a Galois invariant isomorphism $\phi: \mathcal{F}_{L} \rightarrow \mathcal{A}_{2}$, which on the open subset $\mathcal{E}_{E_{8}, E_{7}}$ restricts to the inverse of the explicit isomorphism $\psi$ : $\mathcal{M}_{2} \rightarrow \mathcal{E}_{E_{8}, E_{7}}$ given by Theorem 5 .

For a Hodge-theoretic approach to this isomorphism of moduli spaces, see [Gritsenko and Nikulin 1997, pp. 186-188].

\section{Humbert surfaces and Hilbert modular surfaces}

We next discuss moduli spaces of abelian surfaces with real multiplication. As above, let $D>0$ be a fundamental discriminant, i.e., $D=d$ for $d \equiv 1(\bmod 4)$ or $D=4 d$ for $d \equiv 2,3(\bmod 4)$, where $d>1$ is squarefree in both cases. Then $D$ is the discriminant of the ring of integers $\mathcal{O}_{D}=\mathbb{Z}+\mathbb{Z}(D+\sqrt{D}) / 2$ of the real quadratic field $K=\mathbb{Q}(\sqrt{D})$. Let $\sigma_{1}, \sigma_{2}$ be the two embeddings of $K$ into $\mathbb{C}$. Then $\mathrm{SL}_{2}\left(\mathcal{O}_{D}\right) /\{ \pm 1\}$ acts on $\mathcal{H}^{+} \times \mathcal{H}^{-}$by

$$
\left(\begin{array}{ll}
a & b \\
c & d
\end{array}\right):\left(z_{1}, z_{2}\right) \mapsto\left(\frac{\sigma_{1}(a) z+\sigma_{1}(b)}{\sigma_{1}(c) z+\sigma_{1}(d)}, \frac{\sigma_{2}(a) z+\sigma_{2}(b)}{\sigma_{2}(c) z+\sigma_{2}(d)}\right),
$$

where $\mathcal{H}^{+}=\{z \in \mathbb{C} \mid \operatorname{Im} z>0\}$ is the complex upper half-plane and $\mathcal{H}^{-}=-\mathcal{H}^{+}$is the lower half-plane.

Let

$$
\mathrm{SL}_{2}\left(\mathcal{O}_{D}, \mathcal{O}_{D}^{*}\right)=\left\{\left(\begin{array}{ll}
a & b \\
c & d
\end{array}\right) \in \mathrm{SL}_{2}(K) \mid a, d \in \mathcal{O}_{D}, c \in \mathcal{O}_{D}^{*}, b \in\left(\mathcal{O}_{D}^{*}\right)^{-1}\right\} .
$$

We claim that this action is equivalent to the action of $\operatorname{SL}_{2}\left(\mathcal{O}_{D}, \mathcal{O}_{D}^{*}\right)$ on $\mathcal{H}^{+} \times \mathcal{H}^{+}$. Here $\mathcal{O}_{D}^{*}$ is the dual of $\mathcal{O}_{D}$ with respect to the trace form on $K$ (that is, $\mathcal{O}_{D}^{*}$ is the inverse different of $K$ ). It is an invertible $\mathcal{O}_{D}$-module of rank 1 ; in fact, it is easily checked to be $(1 / \sqrt{D}) \mathcal{O}_{D}$. Assume, without loss of generality, that $\sigma_{1}(\sqrt{D})>0$ and $\sigma_{2}(\sqrt{D})<0$. Then if we let

$$
\psi: \mathcal{H}^{+} \times \mathcal{H}^{-} \rightarrow \mathcal{H}^{+} \times \mathcal{H}^{+}
$$

be the biholomorphic map $\left(z_{1}, z_{2}\right) \mapsto\left(z_{1} \sigma_{1}(\sqrt{D}), z_{2} \sigma_{2}(\sqrt{D})\right)$, and 


$$
\phi: \mathrm{SL}_{2}\left(\mathcal{O}_{D}\right) \rightarrow \mathrm{SL}_{2}\left(\mathcal{O}_{D}, \mathcal{O}_{D}^{*}\right)
$$

be the group isomorphism given by

$$
\left(\begin{array}{ll}
a & b \\
c & d
\end{array}\right) \mapsto\left(\begin{array}{cc}
a & b \sqrt{D} \\
c / \sqrt{D} & d
\end{array}\right)
$$

an easy check shows that the following diagram commutes.

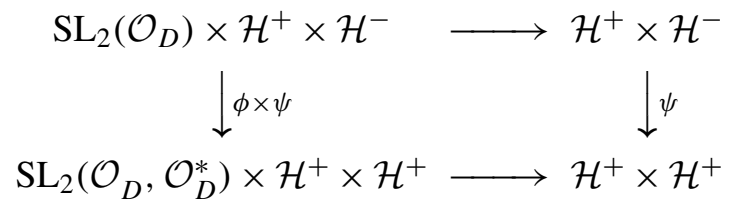

Therefore, $\psi$ induces an isomorphism on the quotients, as desired.

Next, we outline the proof that $\operatorname{SL}_{2}\left(\mathcal{O}_{D}, \mathcal{O}_{D}^{*}\right)$ is the coarse moduli space of principally polarized abelian surfaces with real multiplication by $\mathcal{O}_{D}$, closely following [Hirzebruch and van der Geer 1981]. Let $M=\mathcal{O}_{D} \oplus \mathcal{O}_{D}^{*}$, and define an alternating $\mathbb{Z}$-valued form on $M$ by

$$
E_{M}\left(\left(\alpha_{1}, \beta_{1}\right),\left(\alpha_{2}, \beta_{2}\right)\right)=\operatorname{Tr}_{K / \mathbb{Q}}\left(\alpha_{1} \beta_{2}-\alpha_{2} \beta_{1}\right) .
$$

Now, for $z=\left(z_{1}, z_{2}\right) \in \mathcal{H}^{2}$, consider the embedding

$$
\begin{aligned}
L_{z}: K \oplus K & \rightarrow V=\mathbb{C}^{2}, \\
(\alpha, \beta) & \mapsto \alpha z+\beta=\left(\sigma_{1}(\alpha) z+\sigma_{1}(\beta), \sigma_{2}(\alpha) z+\sigma_{2}(\beta)\right),
\end{aligned}
$$

which gives us a lattice $L_{z}(M)$ in $V$. We use this to transfer $E_{M}$ to $L_{z}(M)$ and extend the form $\mathbb{R}$-linearly. This gives an alternating form

$$
E_{M, z}: V \times V \rightarrow \mathbb{R}
$$

which can be described in coordinates on $\mathbb{C}^{2}$ as

$$
E_{M, z}\left(\left(\zeta_{1}, \zeta_{2}\right),\left(\eta_{1}, \eta_{2}\right)\right)=\frac{\operatorname{Im} \zeta_{1} \overline{\eta_{1}}}{\operatorname{Im} z_{1}}+\frac{\operatorname{Im} \zeta_{2} \overline{\eta_{2}}}{\operatorname{Im} z_{2}}
$$

This gives a Riemann form on the resulting complex torus $V / L_{z}(M)$, and since the form $E_{M}$ on the lattice $L_{z}(M)$ is unimodular, we obtain an abelian variety with principal polarization. The action of $\mathcal{O}_{D}$ is as follows:

$$
\iota(\alpha)\left(\zeta_{1}, \zeta_{2}\right) \mapsto\left(\sigma_{1}(\alpha) \zeta_{1}, \sigma_{2}(\alpha) \zeta_{2}\right) .
$$

Conversely, it is not hard to show that any principally polarized abelian surface with real multiplication by $\mathcal{O}_{D}$ can be identified with some $V / L_{z}(M)$. Finally, we note that the abelian surfaces corresponding to two different $z \in \mathcal{H}^{+} \times \mathcal{H}^{+}$are isomorphic (with an $\mathcal{O}_{D}$-equivariant isomorphism) exactly when these two points differ by an element of $\mathrm{SL}_{2}\left(\mathcal{O}_{D}, \mathcal{O}_{D}^{*}\right)$. Therefore, it follows that the moduli space 
of abelian surfaces with real multiplication is $\operatorname{SL}_{2}\left(\mathcal{O}_{D}, \mathcal{O}_{D}^{*}\right) \backslash\left(\mathcal{H}^{+} \times \mathcal{H}^{+}\right)$, which as we have shown above, is biholomorphic with $\mathrm{SL}_{2}\left(\mathcal{O}_{D}\right) \backslash\left(\mathcal{H}^{+} \times \mathcal{H}^{-}\right)$.

The construction above yields a map from $\mathrm{SL}_{2}\left(\mathcal{O}_{D}\right) \backslash\left(\mathcal{H}^{+} \times \mathcal{H}^{-}\right)$to the quotient of $\mathcal{S}_{2}$, the Siegel upper half-space of degree 2, by the arithmetic group $\mathrm{Sp}_{4}(\mathbb{Z})$. In other words, we get a holomorphic map to $\mathcal{A}_{2}$, the moduli space of principally polarized abelian surfaces. Its image (or its closure in $\mathcal{A}_{2}$ ) is the Humbert surface $\mathcal{H}_{D}$ for discriminant $D$. We next show that the map from the Hilbert modular surface $\mathrm{SL}_{2}\left(\mathcal{O}_{D}\right) \backslash\left(\mathcal{H}^{+} \times \mathcal{H}^{-}\right)$to the Humbert surface is generically two-to-one. We may compute this degree above a very general point on the Humbert surface. Such a point corresponds to a principally polarized abelian surface $A$, where one has forgotten the action of $\mathcal{O}_{D}$ by endomorphisms. Generically, there are exactly two ways to extend the obvious map $\mathbb{Z} \rightarrow \operatorname{End}(A) \cong \mathcal{O}_{D}$ to $\mathcal{O}_{D}$, corresponding to the choice of image of $(D+\sqrt{D}) / 2$.

Our approach to computing equations of Hilbert modular surfaces begins as follows. Fix a discriminant $D$, which we assume to be a fundamental discriminant. First, we need to compute a model of the Humbert surface, i.e., the subvariety of $\mathcal{A}_{2}$ corresponding to abelian surfaces with real multiplication by $\mathcal{O}_{D}$. Via the inverse of the isomorphism $\phi: \mathcal{F}_{L} \rightarrow \mathcal{A}_{2}$ of Section 2.4 above, the Humbert surface corresponds to a surface inside the 3 -dimensional moduli space of $L$-polarized K3 surfaces.

Define a pairing on $\mathcal{O}_{D}$ by $(\alpha, \beta) \mapsto \operatorname{tr}\left(\alpha \beta^{*}\right)$, where $\beta^{*}$ is the Galois conjugate of $\beta$. This gives $\mathcal{O}_{D}$ the structure of an indefinite lattice, which we next identify with the Néron-Severi lattice of $A$.

Proposition 7. Let $A$ be a principally polarized abelian surface with $\operatorname{End}(A) \cong \mathcal{O}_{D}$. Then $\mathrm{NS}(A) \cong \operatorname{End}(A)$. The lattice $\mathrm{NS}(A)$ has a basis with Gram matrix

$$
\left(\begin{array}{cc}
2 & D \\
D & \left(D^{2}-D\right) / 2
\end{array}\right)
$$

of signature $(1,1)$ and discriminant $-D$.

Proof. For a principally polarized abelian surface $A$, there is an isomorphism

$$
\mathrm{NS}(A) \rightarrow(\operatorname{End}(A))^{\dagger}
$$

induced naturally by the polarization, where $\dagger$ is the Rosati involution (also arising from the polarization). Note that for a general polarization one only gets a weaker isomorphism, between the $\mathbb{Q}$-spans of both sides. For the proof of both assertions, see Proposition 5.2.1 in [Birkenhake and Lange 2004]. Now, the Rosati involution is a positive involution of the real quadratic field $\operatorname{End}(A) \otimes \mathbb{Q}$, and hence cannot be the nontrivial element of the Galois group. It must therefore be the identity, whence the subring of $\operatorname{End}(A)$ fixed by $\dagger$ is $\operatorname{End}(A)$ itself, proving the first statement. The 
isomorphism of groups $\mathrm{NS}(A) \rightarrow \operatorname{End}(A)$ is an isometry, taking the intersection form on $\operatorname{NS}(A)$ to the form $(\phi, \psi) \mapsto \operatorname{tr}\left(\phi \psi^{\vee}\right)$ on $\operatorname{End}(A)$, which becomes $(\alpha, \beta) \mapsto$ $\operatorname{tr}\left(\alpha \beta^{*}\right)$ on $\mathcal{O}_{D}$. Computing the matrix of this form on the basis $(1,(D+\sqrt{D}) / 2)$ of $\mathcal{O}_{D}$, we obtain the claimed Gram matrix (2).

We henceforth use $\mathcal{O}_{D}$ also to denote the lattice with underlying group $\mathcal{O}_{D} \cong \mathbb{Z}^{2}$ and form $(\alpha, \beta) \mapsto \operatorname{tr}\left(\alpha \beta^{*}\right)$, with Gram matrix (2).

Proposition 8. There is a primitive embedding, unique up to isomorphism, of the lattice $\mathcal{O}_{D}$ into $U^{3}$. Let $T_{D}$ be the orthogonal complement of $\mathcal{O}_{D}$ in $U^{3}$. Then there is a primitive embedding, unique up to isomorphism, of $T_{D}$ into the $\mathrm{K} 3$ lattice $\Lambda$.

Remark. In the present paper we analyze only fundamental discriminants $D$, for which every embedding into an even lattice of $\mathcal{O}_{D}$ and $T_{D}$ (and of the lattice $L_{D}$, to be introduced before the next theorem) is automatically primitive. But the condition of primitivity is necessary to extend our theoretical analysis also to non-fundamental $D$.

Proof. Since $\mathcal{O}_{D}$ is 2-dimensional, the number of generators $\ell\left(\mathcal{O}_{D}^{*} / \mathcal{O}_{D}\right)$ of the discriminant group is at most 2. In fact, the discriminant group is cyclic of order $D$ if $D$ is odd, and isomorphic to $(\mathbb{Z} / 2 \mathbb{Z}) \oplus\left(\mathbb{Z} /\left(\frac{D}{2} \mathbb{Z}\right)\right)$ if $D$ is even. Therefore, by [Nikulin $1979 \mathrm{~b}$, Theorem 1.14.4], $\mathcal{O}_{D}$ has a unique embedding into the even unimodular lattice $U^{3}$. Let the orthogonal complement be $T_{D}$; it has signature $(2,2)$ and the same discriminant group. By another application of [Nikulin 1979b, Theorem 1.14.4], we see that $T_{D}$ has a unique embedding into $\Lambda_{\mathrm{K} 3}$.

Let $q_{D}$ be the discriminant form of $\mathcal{O}_{D}$, and let $L_{D}$ be the orthogonal complement of $T_{D}$ in $\Lambda$. Then $L_{D}$ has signature $(1,17)$ and discriminant form $q_{D}$. By [Nikulin 1979b, Theorem 1.13.2], $L_{D}$ is characterized uniquely by its signature and discriminant form. Since $\Lambda \cong U^{3} \oplus E_{8}(-1)^{2}$, it is clear that $L_{D} \cong E_{8}(-1)^{2} \oplus \mathcal{O}_{D}$. Finally, $L_{D}$ has a primitive embedding in $\Lambda$, which (again by [Nikulin 1979b, Theorem 1.14.4]) is unique up to isomorphism.

Theorem 9. Let $\mathcal{F}_{L_{D}}$ be the moduli space of $\mathrm{K} 3$ surfaces that are lattice polarized by $L_{D}$. Then the isomorphism $\phi: \mathcal{F}_{L} \rightarrow \mathcal{A}_{2}$ of Section 2.4 induces a birational surjective morphism $\mathcal{F}_{L_{D}} \rightarrow \mathcal{H}_{D}$.

Proof. First, we note that $\mathbb{Z} \subset \mathcal{O}_{D}$ induces an embedding of lattices $\langle 2\rangle \subset \mathcal{O}_{D}$, and therefore an embedding $T_{D} \subset U^{2} \oplus\langle-2\rangle$ of orthogonal complements. Taking orthogonal complements once more in $\Lambda_{\mathrm{K} 3}$, we deduce $L \subset L_{D}$.

Fix embeddings $L \subset L_{D} \subset \Lambda_{\mathrm{K} 3}$. Then we have $L_{D}^{\perp} \hookrightarrow L^{\perp}$, which induces a map $\Omega_{L_{D}} \hookrightarrow \Omega_{L}$ of period domains. We also have a map $\Gamma_{L_{D}} \subset \Gamma_{L}$. These induce a map $\mathcal{F}_{L_{D}}=\Gamma_{L_{D}} \backslash \Omega_{L_{D}} \stackrel{\beta_{D}}{\rightarrow} \Gamma_{L} \backslash \Omega_{L}=\mathcal{F}_{L}$. We will use the fact that this morphism has degree 1 onto the image (i.e., it is an embedding on the generic point of $\mathcal{F}_{L_{D}}$ ). We postpone the proof of this fact until the conclusion of the present argument. 
Let $\phi_{D}: \mathcal{F}_{L_{D}} \stackrel{\beta_{D}}{\rightarrow} \mathcal{F}_{L} \stackrel{\phi}{\rightarrow} \mathcal{A}_{2}$ be the composition of the maps above. Now suppose $X$ is a K3 surface corresponding to a point $p$ in the image of $\beta_{D}$. Then we have $\mathrm{NS}(X) \supset L_{D} \cong E_{8}(-1)^{2} \oplus \mathcal{O}_{D}$, and therefore $T_{X} \subset T_{D}$. If $A$ (corresponding to $\phi(p))$ is the abelian surface connected to $X$ through a Shioda-Inose structure, we have $T_{A} \cong T_{X} \subset T_{D}$. Therefore, $\mathrm{NS}(A) \supset \mathcal{O}_{D}$, the orthogonal complement of $T_{D}$ in $U^{3}$. Therefore $\operatorname{End}(A)^{\dagger} \supset \mathcal{O}_{D}$, and $\phi(p)$ must lie on the Humbert surface $\mathcal{H}_{D}$. This proves that the image of $\mathcal{F}_{L_{D}}$ lands in $\mathcal{H}_{D}$.

Conversely, suppose $q$ is a point on $\mathcal{H}_{D}$ corresponding to an abelian surface $A$ with real multiplication by $\mathcal{O}_{D}$. Then $\operatorname{End}(A) \supset \mathcal{O}_{D}$, and since the Rosati involution (being positive) can only act as the trivial element of $\mathbb{Q}(\sqrt{D})$, we must have $\operatorname{End}(A)^{\dagger} \supset \mathcal{O}_{D}$. Retracing the argument in the previous paragraph, we see $p=\phi^{-1}(q)$ must have $\operatorname{NS}(X) \supset L_{D}$, and therefore is in the image of $\beta_{D}$. This proves that $\phi \circ \beta_{D}$ is surjective onto $\mathcal{H}_{D}$.

Since $\phi$ is an isomorphism and $\beta_{D}$ has degree 1 onto its image, so does $\phi \circ \beta_{D}$.

We now prove that fact used above: $\beta_{D}$ is generically an embedding.

\section{Proposition 10. The map $\mathcal{F}_{L_{D}} \rightarrow \mathcal{F}_{L}$ is generically an embedding.}

Proof. To see this, we claim that it is enough to show that there is an element $\gamma_{0} \in O(\Lambda)$ such that $\gamma_{0}$ fixes $L$ pointwise, preserves the sublattice $L_{D}$, and acts by -1 on $L^{\perp}$. For suppose we have $x=\gamma y$ with $x, y \in \Omega_{L_{D}}$ and $\gamma \in \Gamma_{L}$, with $x$ and $y$ very general. Then we would like to show that in fact we already have $x=\gamma^{\prime} y$ with $\gamma^{\prime} \in \Gamma_{L_{D}}$. Then $\Lambda_{x}^{1,1}:=\{z \in x \mid\langle z, x\rangle=0\}$ and $\Lambda_{y}^{1,1}$ both equal $L_{D}$, since $x$ and $y$ are very general, and therefore $\gamma$ preserves $L_{D}$ (though it might not fix this lattice pointwise). If $\gamma$ acts trivially on $L_{D}$, we may take $\gamma^{\prime}=\gamma$, and are done. If not, then $\gamma$ must act by -1 on $L_{D} / L \cong \mathbb{Z}$. Then $\gamma^{\prime}=\gamma_{0} \gamma$ will suffice.

To prove the claim, we may consider specific embeddings $L \subset L_{D} \subset \Lambda$. If $D \equiv 0(\bmod 4)$, then $L_{D} \cong E_{7}(-1) \oplus\langle-D / 2\rangle \oplus U \oplus E_{8}(-1)$. We may embed it inside $\left(E_{8}(-1) \oplus U \oplus U\right) \oplus\left(U \oplus E_{8}(-1)\right)$ by embedding $E_{7}(-1)$ inside $E_{8}(-1)$ as the orthogonal complement of a root $\delta$, and $\langle-D / 2\rangle$ primitively inside $U \oplus U$. Let $\gamma_{0}$ be the automorphism of $\Lambda$ which acts on the first copy of $E_{8}(-1)$ as the reflection $s_{\delta}$ in the hyperplane orthogonal to $\delta$, by -1 on $U \oplus U$, and as the identity on the part $U \oplus E_{8}(-1)$.

If $D \equiv 1(\bmod 4)$, consider a system of simple roots in $E_{8}(-1)$ whose intersections give the standard Dynkin matrix. Let $\alpha$ be the simple root such that if we remove the corresponding vertex from the Dynkin diagram, we obtain the Dynkin diagram for $E_{7}(-1)$. The orthogonal complement of this copy of $E_{7}(-1)$ is some root $\delta$. Let $\beta \in U$ be any element of norm $(1-D) / 2$. It is easy to check that the copy of $E_{7}(-1)$ and $\alpha+\beta$ generate a negative definite lattice $M$ of discriminant $D$, and that $U \oplus M \oplus E_{8}(-1)$ is isometric to $L_{D}$ (since it is has the right signature and discriminant). In other words, the diagram of embeddings 


$$
E_{7}(-1) \hookrightarrow M \subset E_{8}(-1) \oplus U \hookrightarrow E_{8}(-1) \oplus U^{2}
$$

extends on adding a factor of $U \oplus E_{8}(-1)$ to

$$
\begin{aligned}
L=E_{7}(-1) \oplus U \oplus E_{8}(-1) \hookrightarrow M \oplus U \oplus E_{8}(-1) & =L_{D} \subset E_{8}(-1) \oplus U^{2} \oplus E_{8}(-1) \\
& \rightarrow E_{8}(-1) \oplus U^{3} \oplus E_{8}(-1)=\Lambda .
\end{aligned}
$$

Now, consider the automorphism $\gamma_{0}$ of $\Lambda \cong E_{8}(-1) \oplus U^{3} \oplus E_{8}(-1)$ given by $\left(s_{\delta},-\left.1\right|_{U},-\left.1\right|_{U}, 1_{U}, 1_{E_{8}(-1)}\right)$. By construction, $\gamma_{0}$ fixes $L$ pointwise, and acts by -1 on $L^{\perp}$. We need to show that $\gamma_{0}$ preserves $L_{D}$, or equivalently, that it preserves $M$. We know that $M$ is spanned by $E_{7}(-1)$ (which is fixed by $\gamma_{0}$ ) and $\alpha+\beta$. Now, $\gamma_{0}$ takes $\beta$ to $-\beta$, by construction. Also, $\alpha+\gamma_{0}(\alpha)=\alpha+s_{\delta}(\alpha) \in E_{8}(-1)$ is invariant by $s_{\delta}$, and is therefore in the orthogonal complement $E_{7}(-1)$ of $\delta$. Therefore $\gamma_{0}(\alpha) \in-\alpha+E_{7}(-1)$ and $\gamma_{0}(\alpha+\beta) \equiv-(\alpha+\beta) \bmod E_{7}(-1)$. So $\gamma_{0}$ preserves the lattice $M$.

Next, we want to understand the Hilbert modular surface $Y_{-}(D)$, which is a double cover of $\mathcal{H}_{D}$. First, we must identify the branch locus. Since the map $Y_{-}(D) \rightarrow \mathcal{H}_{D}$ is obtained by simply forgetting the action of $e_{D}=(D+\sqrt{D}) / 2$ on the abelian surface, the branch locus is the subvariety $W$ of $\mathcal{H}_{D}$ corresponding to abelian surfaces $A$ such that $e_{D}=(D+\sqrt{D}) / 2$ and $e_{D}^{\prime}=(D-\sqrt{D}) / 2$ are conjugate in the endomorphism ring, say by $\iota \in \operatorname{End}(A)$. It follows that $\iota^{2}$ fixes $\mathcal{O}_{D}$ pointwise. Generically this implies $\iota^{2}= \pm 1$, and $\iota e_{D}=e_{D}^{\prime} \iota$. This shows that $\operatorname{End}(A)$ for such $A$ is generically an order in a quaternion algebra $B$.

In fact, the branch locus corresponds to the case when we have a split quaternion algebra, i.e., $\iota^{2}=1$. Then $A$ is isogenous over $\overline{\mathbb{Q}}$ to the square of an elliptic curve. To see this fact, observe that the map $\Gamma \backslash \mathcal{H}^{2} \rightarrow \mathrm{Sp}_{4}(\mathbb{Z}) \backslash \mathcal{S}_{2}\left(\right.$ where $\Gamma=\operatorname{SL}_{2}\left(\mathcal{O}_{D}, \mathcal{O}_{D}^{*}\right)$ ) factors through the quotient $(\Gamma \cup \Gamma \sigma) \backslash \mathcal{H}^{2}$, where $\sigma$ is the involution $\left(z_{1}, z_{2}\right) \mapsto$ $\left(z_{2}, z_{1}\right)$ exchanging the two factors of $\mathcal{H}^{2}$, and the induced map on this quotient is generically one-to-one [Hirzebruch and van der Geer 1981, p. 158]. Therefore, to understand the branch locus, we need to understand the 1-dimensional part of the fixed point set of $\sigma$ on $\Gamma \backslash \mathcal{H}^{2}$. This (and more) was done by Hausmann [1982, p. 35]. The result is that the fixed point set consists of a small number of explicit modular curves $F_{w}$ for $w \in\{1,4, D, D / 4\}$ (some of these may be empty, and when they are nonempty, these curves are irreducible). A simple explicit analysis of the condition relating $z_{1}$ and $z_{2}$ on these curves reveals that the generic point on each of these corresponds to an abelian surface whose ring of endomorphisms contains zero divisors, and is therefore a split quaternion algebra. For instance, the image of the diagonal of $\mathcal{H}^{2}$ (given by $z_{1}=z_{2}$ ) in $\Gamma \backslash \mathcal{H}^{2}$ is an obvious component of the branch locus. For the corresponding abelian surface, as constructed earlier in this section, the map $\left(\zeta_{1}, \zeta_{2}\right) \mapsto\left(\zeta_{2}, \zeta_{1}\right)$ is a holomorphic involution, showing that the endomorphism algebra is split. For completeness, we prove this next. 
Proposition 11. Let $\Gamma=\mathrm{SL}_{2}\left(\mathcal{O}_{D}, \mathcal{O}_{D}^{*}\right)$ and let $C$ be one of the curves $F_{w}$ for $w \in\{1, D\}$ if $D$ is odd, $w \in\{1,4, D / 4, D\}$ if $D$ is even. The generic point on $C$ corresponds to an abelian surface whose algebra of endomorphisms is a split quaternion algebra.

Proof. We use the notation of [van der Geer 1988, Chapter V]. The ideal $\mathfrak{a}=\mathcal{O}_{D}^{*}$ has norm $A=1 / D$. In the reference, it is assumed that $\mathfrak{a}$ is an integral ideal, but we may for instance replace $\mathcal{O}_{D}^{*}$ by the integral $D \mathcal{O}_{D}^{*}=\sqrt{D} \mathcal{O}_{D}$ without loss of generality in Hausmann's proof. This would replace $A$ by $D^{2} A=D$, and would not affect any of the arguments below, which depend only on the square class of $A$. Proposition V.1.5 of [van der Geer 1988] states that a point on $F_{N}$ corresponds to an abelian surface whose endomorphism algebra is isomorphic to the indefinite quaternion algebra

$$
Q_{N}=\left(\frac{D,-N /(A D)}{\mathbb{Q}}\right),
$$

while Lemma V.1.4 in the book says that $F_{N}$ is nonempty if and only if for each prime $q$ dividing $D$ and not dividing $N$, we have $\chi_{D(q)}(N)=(A, D)_{q}$. For an explanation of the notation, see [van der Geer 1988, pp. 2-3]. In our situation, we have $A D=1$. If $N=D$, we get the algebra $\left(\frac{D,-D}{\mathbb{Q}}\right)$, which is obviously split. This argument also takes care of $N=D / 4$ when $D$ is even. Next, suppose $N=1$. Then $F_{1}$ is nonempty if and only if for every prime dividing $D$, we have $(D, D)_{q}=1$. So

$$
1=(D, D)_{q}=(D,-D)_{q} \cdot(D,-1)_{q}=(D,-1)_{q} .
$$

It follows that the quaternion algebra $Q_{1}=\left(\frac{D,-1}{\mathbb{Q}}\right)$ is split. The proof for $N=4$ is similar.

Remark 12. A further analysis of the proof above reveals the number of components of the branch locus, which is corroborated by the calculations in this paper.

Coming back to our analysis of the endomorphisms of the abelian surfaces corresponding to points on the branch locus, we note that the Rosati involution must fix $\mathcal{O}_{D}$ and $\iota$, by positivity. Consider the form $(x, y) \mapsto \operatorname{Tr}(x \bar{y})$, where ${ }^{-}: B \rightarrow B$ is the natural involution taking $\sqrt{D}$ to its negative and $\iota$ to its negative, and $\operatorname{Tr}$ is the reduced trace. The matrix of this form acting on $\mathcal{O}_{D}+\mathbb{Z} \iota$ is

$$
\left(\begin{array}{ccr}
2 & D & 0 \\
D & \left(D^{2}-D\right) / 2 & 0 \\
0 & 0 & -2
\end{array}\right),
$$

which therefore gives an even lattice of signature $(1,2)$ and discriminant $2 D$. We claim that when $D$ is fundamental, the index, call it $c$, of $\mathcal{O}_{D} \oplus \mathbb{Z} \iota$ as a sublattice of $\operatorname{End}(A)$ is 1 or 2, with $c=2$ possible only if $4 \mid D$. Indeed $\operatorname{End}(A)$ is an even 
lattice of rank 3 and discriminant $2 D / c^{2}$. But the discriminant is an even integer because the rank is odd, and $D$ has no repeated prime factors except possibly $2^{2}$ or $2^{3}$. Thus as claimed the index $c$ must be either 1 or 2 , with $c=2 \Rightarrow 4 \mid D$. Therefore, the only possibilities for the discriminant of the resulting Néron-Severi group for a point corresponding to the branch locus are $2 D$ and $D / 2$. This is also the discriminant of the transcendental lattice of the abelian surface, and by the Shioda-Inose structure, the corresponding K3 surface also has a Néron-Severi lattice of rank 19 and discriminant $2 D$ or $D / 2$, and contains $L_{D} \cong E_{8}(-1)^{2} \oplus \mathcal{O}_{D}$.

Finally, we mention that a group of involutions acts naturally on the Hilbert modular surface $Y_{-}(D)$. This group is isomorphic to $(\mathbb{Z} / 2 \mathbb{Z})^{t-1}$, where $t$ is the number of distinct primes dividing $D$. These extra involutions arise from the Hurwitz-Maass extension of $\mathrm{PSL}_{2}\left(\mathcal{O}_{D}, \mathcal{O}_{D}^{*}\right)$. For more details see [van der Geer 1988, Section I.4].

\section{Method of computation}

We now outline our method to obtain equations for the Hilbert modular surfaces $Y_{-}(D)$ for fundamental discriminants $D$.

Step 1. We compute the unique lattice $L_{D}$, writing it in the form $U \oplus N$ (we know $L_{D}$ contains a copy of $U$, since $L=U \oplus E_{8}(-1) \oplus E_{7}(-1)$ does, and $L \supset L_{D}$ ). Note that $N$ is not uniquely determined, and it turns out to be useful to choose $N$ such that its root lattice $R$ has small codimension in $N$, and such that $N / R$ has a basis consisting of vectors of small (fractional) norm. One may then attempt to realize $L_{D}$ as the Néron-Severi lattice of an elliptic K3 surface with reducible fibers corresponding to the irreducible root lattices in $R$. The remaining generators of the Néron-Severi lattice should then arise as sections (of small height) in the Mordell-Weil group of the elliptic fibration. Of course, we need to make sure that $N$ contains $E_{8} \oplus E_{7}$, but this is easily achieved by showing that the dual lattice $N^{*}$ contains a vector of norm $2 / D$.

For most of the examples in this paper, we were able to describe a family of elliptic K3 surfaces with Néron-Severi lattice $L_{D}$ and with Mordell-Weil rank 0 or 1 . This construction gives us a Zariski-open subset of $\mathcal{F}_{L_{D}}$, with some possible missing curves corresponding to jumps in the Mordell-Weil ranks or the ranks of the reducible fibers, or to denominators introduced in the parametrization process.

For all the examples we considered, the moduli space $\mathcal{F}_{L_{D}}$ turns out to be rational (i.e., birational with $\mathbb{P}^{2}$ over $\mathbb{Q}$ ). This property will fail to hold once $D$ is large enough, but there is still some hope of writing down usable equations for $\mathcal{F}_{L_{D}}$ if it is not too complicated.

Suppose that we have exhibited a Zariski open subset of $\mathcal{F}_{L_{D}}$ as a Zariski open subset $U=\mathbb{P}_{r, s}^{2} \backslash V$ of the projective plane. 
Step 2. We find a different elliptic fibration on the generic member of the family of $\mathrm{K} 3$ surfaces in Step 1, with reducible fibers of types $E_{8}$ at $t=\infty$ and $E_{7}$ at $t=0$. This is accomplished by 2- and 3-neighbor steps, which we shall describe in the next section.

Once we obtain the alternate elliptic fibration, we may compute the map from $U$ to $\mathcal{A}_{2}$ by the explicit formulas of Section 2.4. This gives us the Igusa-Clebsch invariants of the associated genus-2 curve, in terms of the two parameters $r$ and $s$.

Step 3. Consider the base change diagram

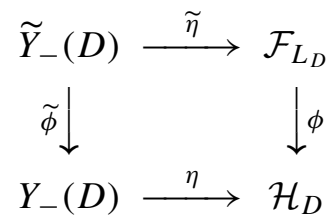

Since $\phi$ is a birational map, so is $\widetilde{\phi}$. We want to describe the degree-2 map $\widetilde{\eta}$. In the current situation, we must have a model for $\widetilde{Y}_{-}(D)$ (which is birational to $Y_{-}(D)$ ) of the form

$$
z^{2}=f(r, s),
$$

where $f(r, s)=0$ describes the branch locus of $\tilde{\eta}$. As we have seen, this locus $f(r, s)=0$ must be the union of some irreducible curves, which are either components of the excluded locus $V$ above, or belong to the sublocus of $U$ where the Picard number jumps by 1 , the discriminant changing to $2 D$ or $D / 2$. There are finitely many possibilities for how this may happen. For instance, the elliptic fibration may have an extra $A_{1}$ fiber, or a $D_{6}$ fiber may get promoted to an $E_{7}$ fiber, or the surface may have a new section that raises the Mordell-Weil rank. Similarly, in any instance, we may easily list the allowed changes in the reducible fibers, or the allowed heights and intersections with components of reducible fibers of a new section of the fibration. Hence we get a list of polynomials $f_{1}(r, s), f_{2}(r, s), \ldots, f_{k}(r, s) \in \mathbb{Z}[r, s]$ whose zero loci are the possible components of the branch locus. We may assume that each $f_{i}(r, s)$ has content 1 .

We therefore have the following model for $\widetilde{Y}_{-}(D)$ as a double cover of $\mathbb{P}_{r, s}^{2}$ :

$$
z^{2}=f(r, s)=C f_{i_{1}}(r, s) f_{i_{2}}(r, s) \ldots f_{i_{m}}(r, s)
$$

for some subset $\left\{i_{1}, \ldots, i_{m}\right\} \subseteq\{1, \ldots, k\}$ and some squarefree integer $C$.

Step 4. In the final step, we show how to compute the subset $I=\left\{i_{1}, \ldots, i_{m}\right\}$ and the twist $C$. First, we note that the Hilbert modular surface $Y_{-}(D)$ has good reduction outside primes dividing $D$, by [Rapoport 1978; Deligne and Pappas 1994]. Therefore, there are only finitely many choices for $C$ as well. Now we check each of these choices by computer, using the method described in the next paragraph, and rule out all but one. 
So suppose we have a putative choice of $C$ and $I$. We proceed to check whether this choice of twist is correct by reduction modulo several odd primes $p$ that do not divide the discriminant $D$. We specialize $r, s$ to elements of $\mathbb{F}_{p}$, and by the formulas of Step 2, obtain Igusa-Clebsch invariants for in weighted projective space over $\mathbb{F}_{p}$. Since the Brauer obstruction vanishes for finite fields, we can construct a curve $C_{r, s}$ over $\mathbb{F}_{p}$.

We may use the following lemma to detect whether the Jacobian of $C_{r, s}$ has real multiplication defined over $\mathbb{F}_{p}$ or not.

Lemma 13. Let $A$ be an abelian surface over $\mathbb{F}_{p}$, and let $\phi$ be the Frobenius endomorphism of A relative to $\mathbb{F}_{p}$. Suppose that the characteristic polynomial $P(T)$ of $\phi$ is irreducible over $\mathbb{Q}$. Let $Q$ be the symmetric characteristic polynomial of $\phi+p \phi^{-1}$, defined by $P(T)=T^{2} Q\left(T+p T^{-1}\right)$.

(1) If A has real multiplication by an order in $\mathcal{O}_{D}$ defined over $\mathbb{F}_{p}$, then $Q$ is a quadratic polynomial of discriminant $c^{2} D$ for some integer $c$.

(2) If A has real multiplication by an order in $\mathcal{O}_{D}$, defined over $\mathbb{F}_{p^{2}}$, but not over $\mathbb{F}_{p}$, then we have $Q(X)=X^{2}-n$ for some $n \in \mathbb{Z}$ not of the form $c^{2} D$ for any integer $c$.

(3) If $Q(X)$ is a quadratic polynomial of discriminant $D$ (resp. $c^{2} D$ for some positive integer $c$ ), then $A$ has real multiplication by $\mathcal{O}_{D}$ (resp. an order in $\mathcal{O}_{D}$ of conductor dividing $c$ ), defined over $\mathbb{F}_{p}$.

Note that $A$ might simultaneously have real multiplication $\iota: \mathcal{O} \hookrightarrow \operatorname{End}(A)$ and $\iota^{\prime}: \mathcal{O}^{\prime} \hookrightarrow \operatorname{End}(A)$ by two orders of $\mathcal{O}_{D}$, if its ring of endomorphisms is a quaternion algebra. Furthermore, if one of these is defined over $\mathbb{F}_{p}$ and the other only over $\mathbb{F}_{p}^{2}$, then by parts (1) and (2) of the lemma, $Q(X)$ must be of the form $X^{2}-c^{2} D$ for some integer $c$.

We will give the proof of the lemma below, but first we indicate how to use it to find the correct twist. We choose a suitable prime $p \nmid D$, and for some $r, s$ in $\mathbb{F}_{p}$, we calculate the number of points $\bmod p$ and $p^{2}$ of the resulting curve $C_{r, s}$. This is enough to describe the polynomial $Q(X)$ for the abelian surface $A=J(C)$, by the Lefschetz-Grothendieck trace formula. Suppose $P(T)=T^{2} Q\left(T+p T^{-1}\right)$ is irreducible. Then the first hypothesis of the lemma is satisfied. If in addition $Q(X)=X^{2}+a X+b$ has discriminant $D$ and nonzero linear term $a$, then $A$ must have real multiplication defined over $\mathbb{F}_{p}$, by part (3) of the lemma (and the comments following it). In fact, most of the time we can even make do with the weaker assumption that $Q(X)$ has discriminant in the square class of $D$, since (3) guarantees that $A$ has real multiplication by an order in $\mathbb{Q}(\sqrt{D})$. We can compute the discriminant of $\mathrm{NS}(A)$ by computing that of $\mathrm{NS}(Y)$, where $Y$ is the elliptic K3 surface related to $A$ by a Shioda-Inose structure. If this discriminant is $D$, it follows that $A$ must have real multiplication by the full ring of integers $\mathcal{O}_{D}$. 
Now, by the property of a coarse moduli space, $A$ gives rise to an $\mathbb{F}_{p}$-point $(r, s)$ of the Hilbert modular surface $Y_{-}(D)$, i.e., the corresponding point on the Humbert surface must actually lift to the double cover. Therefore, if $C f_{i_{1}}(r, s) \ldots f_{i_{m}}(r, s)$ is not a square, we must have the wrong quadratic twist. We can run this test for many such $(r, s) \in \mathbb{F}_{p} \times \mathbb{F}_{p}$. Note that a single large prime is usually enough to pin down the correct choice of $\left\{i_{1}, \ldots, i_{m}\right\}$, by eliminating all but one possibility. However, to pin down the correct choice of $C$, we may need to use several primes, until we find one for which $C / C^{\prime}$ is not a quadratic residue, where $C^{\prime}$ is the correct twist. However, in any case, this procedure is guaranteed to terminate, and in practice, it terminates fairly quickly.

At the end of Step 4 of the algorithm, we have determined a birational model of $Y_{-}(D)$ over $\mathbb{Q}$. We now give the proof of the lemma above.

Proof of Lemma 13. We will use [Tate 1966a, Theorem 2]. Since $P(T)$ is irreducible, it follows that $F=\mathbb{Q}[\phi]$ is simple, and also that $F=E:=\operatorname{End}_{\mathbb{F}_{p}}(A) \otimes \mathbb{Q}$ is a quartic number field. First, assume that real multiplication by an order $\mathcal{O} \subset \mathcal{O}_{D}$ is defined over $\mathbb{F}_{p^{2}}$ but not over $\mathbb{F}_{p}$. Say $\mathcal{O}$ contains $f \sqrt{D}$ for some positive integer $f$. Then consider the base change of $A$ to $\mathbb{F}_{p^{2}}$. The Frobenius over the new field is $\phi^{2}$, and therefore, $\phi^{2}$ commutes with $f \sqrt{D}$, but $\phi$ must anticommute with $f \sqrt{D}$. Therefore $\mathbb{Q}\left(\phi^{2}\right)$ is a strict subfield of $\mathbb{Q}(\phi)$, and must be quadratic. Hence $\phi^{2}$ satisfies a quadratic equation $\phi^{4}+a \phi^{2}+b=0$, and since this must be the characteristic polynomial of $\phi$, we must have $b=p^{2}$. Therefore

$$
\phi^{2}+p^{2} \phi^{-2}+a=\left(\phi+p \phi^{-1}\right)^{2}+a-2 p=0,
$$

proving the assertion (1).

Now, assume that $A$ has real multiplication by $\mathcal{O} \subset \mathcal{O}_{D}$, defined over $\mathbb{F}_{p}$, and let $f \sqrt{D} \subset \mathcal{O}$, as before. Then $\mathbb{Q}\left(\phi+p \phi^{-1}\right)$ is a quadratic subfield of $\mathbb{Q}(\phi)$. Also, $f \sqrt{D} \in \mathbb{Q}(\phi)$, so we have $f \sqrt{D}=g(\phi)$ for some polynomial $g \in \mathbb{Q}[T]$. Then $f \sqrt{D}$ is its own dual isogeny, and so $f \sqrt{D}=g\left(p \phi^{-1}\right)$ as well. Therefore, $f \sqrt{D}=\frac{1}{2}\left(g(\phi)+g\left(p \phi^{-1}\right)\right)$ can be expressed as $h\left(\phi+p \phi^{-1}\right)$ for some $h \in \mathbb{Q}(T)$. It follows that $\mathbb{Q}(\sqrt{D})=\mathbb{Q}\left(\phi+p \phi^{-1}\right)$ and therefore the minimal polynomial $Q(T)$ of $\phi+p \phi^{-1}$ has discriminant equal to $D$ times a square. This proves (2).

Finally, if $Q$ is a quadratic polynomial of discriminant $c^{2} D$, then $\eta=\phi+p \phi^{-1}$ is an endomorphism of $A$ satisfying $Q(\eta)=0$. Therefore $\mathbb{Z}[\eta] \cong \mathcal{O}:=\mathbb{Z}+c \mathcal{O}_{D}$, and we conclude that $A$ has real multiplication by $\mathcal{O}$ defined over $\mathbb{F}_{p}$, proving (3).

We conclude this section with a few comments on the models of Hilbert modular surfaces computed in this paper. We first give formulas for the family of elliptic K3 surfaces over $\mathcal{F}_{L_{D}}$, and then describe the 2- and 3-neighbor steps necessary to reach the alternate fibration of Step 2. The details of the parametrization will not be included in the paper, but they are available in the online supplement. Steps 
3 and 4 are relatively easy to automate. We simply write down the result, which is the equation of $Y_{-}(D)$ as a double cover of the Humbert surface. We give a table of points of small height for which the Brauer obstruction vanishes, and the associated curves of genus 2. We also analyze the Hilbert modular surface further in the cases that we can describe it as a K3 or honestly elliptic surface. In particular, we determine the (geometric) Picard number and generators for the Mordell-Weil group of sections in most of the cases. We also analyze the branch locus of Step 3, identifying it with a union of quotients of classical modular curves in several cases, with the help of explicit formulas given in [Elkies 1998] or obtained by the methods of that paper. Finally, for many discriminants, we are able to exhibit curves of low genus on the surface, possessing infinitely many rational points.

We found the method of [van Luijk 2007] quite useful in determining the ranks of the Néron-Severi lattices of these surfaces. Briefly, the method is as follows. Let $X$ be a smooth projective surface over $\mathbb{Q}$. For a prime $p$ of good reduction, let $X_{p}$ be the reduction of a good model of $X$ at $p$. By counting points on $X_{p}\left(\mathbb{F}_{q}\right)$ for a small number of prime powers $q=p^{e}$, we obtain the characteristic polynomial of the Frobenius $\phi_{p}$ on some $\ell$-adic étale cohomology group $H^{2}\left(X \times \overline{\mathbb{F}}_{p}, \mathbb{Q}_{l}\right)$. The number of roots $\rho_{0}(p)$ of this polynomial which are $p$ times a root of unity is an upper bound on the geometric Picard number of $X_{p}$. Therefore $\rho(X) \leq \rho_{0}(p)$ for such primes. If we have $\rho_{0}\left(p_{1}\right)=\rho_{0}\left(p_{2}\right)=\rho_{0}$, but the (expected) square classes of the discriminant of the Néron-Severi groups modulo these primes (as predicted by the Artin-Tate formula [Tate 1966b]) are distinct, we may even deduce $\rho(X)<\rho_{0}$. For if $X_{p}$ does not satisfy the Tate conjecture for some $p \in\left\{p_{1}, p_{2}\right\}$, then $\rho(X) \leq \rho\left(X_{p}\right)<\rho_{0}$. On the other hand, if both these reductions satisfy the Tate conjecture, then they also satisfy the Artin-Tate conjecture [Milne 1975a, 1975b], and since the size of the Brauer group of $X_{p}$ is a square [Milne 1975a; Liu et al. 2005], the Néron-Severi groups must have discriminants in the same square class.

\section{Neighbor method}

Finally, we describe how to transform from one elliptic fibration to another, using 2- and 3-neighbor steps. We start with an elliptic K3 surface over a field $k$, which we assume has characteristic different from 2 or 3 . Let $F$ be the class of the fiber, and $O$ be the class of the zero section. The surface $X$ is a minimal proper model of a given Weierstrass equation

$$
y^{2}=x^{3}+a_{2}(t) x^{2}+a_{4}(t) x+a_{6}(t) .
$$

Here the elliptic fibration is $\pi: X \rightarrow \mathbb{P}_{t}^{1}$. Now, let $F^{\prime}$ be an elliptic divisor (i.e., $F^{\prime}$ is effective, $F^{\prime 2}=0$, the components of $F^{\prime}$ are smooth rational curves, and $F^{\prime}$ is primitive). 
We would like to write down the Weierstrass equation for this new elliptic fibration on $X$. The space of global sections $H^{0}\left(X, \mathcal{O}_{X}\left(F^{\prime}\right)\right)$ has dimension 2 over $k$. The ratio of any two linearly independent sections gives us the new elliptic parameter $u$. To compute the space of global sections, we proceed as follows. Any global section gives a section of the generic fiber $E=\pi^{-1}(\eta)$, which is an elliptic curve over $k(t)=k(\eta)$. Therefore, if we have a basis of global sections of $D=F_{\text {hor }, \eta}^{\prime}$ over $k(t)$, say $\left\{s_{1}, \ldots, s_{r}\right\}$ (where $r=h^{0}\left(\mathcal{O}_{E}(D)\right)=\operatorname{deg}(D)=F_{\text {hor }}^{\prime} \cdot F=F^{\prime} \cdot F$ ), we can assume that any global section of $\mathcal{O}_{X}\left(F^{\prime}\right)$ is of the form

$$
b_{1}(t) s_{1}+\cdots+b_{r}(t) s_{r} .
$$

We can now use the information from $F_{\text {ver, }}$, which gives us conditions about the zeroes and poles of the functions $b_{i}$, to find the linear conditions cutting out $H^{0}\left(X, \mathcal{O}_{X}\left(F^{\prime}\right)\right)$, which will be 2-dimensional.

When $F \cdot F^{\prime}=r$, we say that going between these elliptic fibrations is an $r$ neighbor step. We will explain the reason for this terminology shortly. In this paper we use only 2- and 3-neighbor steps. First, we describe how to convert to a genus-1 curve in the case when $E=F_{\text {hor }, \eta}=2 O$ or $3 O$. These are the most familiar cases of the 2- and 3-neighbor steps, and the other cases of the 2-neighbor step that are needed are more exhaustively described in [Kumar 2014].

Suppose $D=2 O$. Then $\{1, x\}$ is a basis of global sections of $\mathcal{O}_{E}(D)$. Therefore, on $X$ we obtain two global sections, 1 and $c(t)+d(t) x$ for some $c(t), d(t) \in k(t)$. The ratio between the two gives the elliptic parameter $u$. We set $x=(u-c(t)) / d(t)$, and substitute into the Weierstrass equation to obtain an equation

$$
y^{2}=g(t, u) .
$$

Because $F^{\prime}$ is an elliptic divisor, the generic fiber of this surface over $\mathbb{P}_{u}^{1}$ is a curve of genus 1 . Thus, once we absorb square factors into $y^{2}$ we obtain an equivalent $g$ that is a polynomial of degree 3 or 4 in $t$. Then $y^{2}=g(t, u)$ is standard form of the equation of a genus- 1 curve as a branched double cover of $\mathbb{P}^{1}$.

Suppose $D=3 O$. Then $\{1, x, y\}$ is a basis of global sections of $\mathcal{O}_{E}(D)$. On $X$ we obtain two global sections, 1 and $c(t)+d(t) x+e(t) y$. We set the ratio equal to $u$, solve for $y$, and substitute into the Weierstrass equation to obtain

$$
(u-c(t)+d(t) x)^{2}=e(t)^{2}\left(x^{3}+a_{2}(t) x^{2}+a_{4}(t) x+a_{6}(t)\right) .
$$

This equation is of degree 3 in $x$, and after some simple algebra (scaling and shifting $x$ ), we may arrange it to have degree 3 in $t$ as well. We end up with a plane cubic curve, which is the standard model of a genus- 1 curve with a degree- 3 line bundle.

Finally, if we also know that the elliptic fibration corresponding to $F^{\prime}$ has a section (which follows in each of our examples by Proposition 4), then it is isomorphic 
to the Jacobian of the genus- 1 curve we have computed. We may use standard formulas to write down the Weierstrass equation of the Jacobian (see [An et al. 2001] and the references cited therein), and this is the desired Weierstrass equation for the elliptic fibration with fiber $F^{\prime}$.

This neighbor step from $F$ to $F^{\prime}$ corresponds to computing an explicit isomorphism between two presentations $\mathbb{Z} O+\mathbb{Z} F+T$ and $\mathbb{Z} O^{\prime}+\mathbb{Z} F^{\prime}+T^{\prime}$ of $\mathrm{NS}(X)$. Note that $T \cong F^{\perp} / \mathbb{Z} F$, where ${ }^{\perp}$ refers to the orthogonal complement in $\operatorname{NS}(X)$. The sublattice $\left(\mathbb{Z} F+\mathbb{Z} F^{\prime}\right)^{\perp}$ projects to an index-r sublattice of both $F^{\perp} / \mathbb{Z} F$ and $F^{\prime \perp} / \mathbb{Z} F^{\prime}$, where $r=F \cdot F^{\prime}$. Therefore these lattices are $r$-neighbors.

\section{Discriminant 5}

6.1. Parametrization. This is the smallest fundamental discriminant for real multiplication, and it is small enough that we do not need any 2- or 3-neighbor steps: we can instead just start with a K3 surface with $E_{8}$ and $E_{7}$ fibers, and ask for the extra condition which allows a section of height $\frac{5}{2}=4-\frac{3}{2}$.

Proposition 14. The moduli space of $\mathrm{K} 3$ surfaces lattice polarized by $L_{5}$, the unique even lattice of signature $(1,17)$ and discriminant 5 containing

$$
U \oplus E_{8}(-1) \oplus E_{7}(-1),
$$

is birational to the projective plane $\mathbb{P}_{g, h}^{2}$. The family of $\mathrm{K} 3$ surfaces is given by the Weierstrass equation

$$
y^{2}=x^{3}+\frac{1}{4} t^{3}\left(-3 g^{2} t+4\right) x-\frac{1}{4} t^{5}\left(4 h^{2} t^{2}+\left(4 h+g^{3}\right) t+(4 g+1)\right) .
$$

Proof. We start with a family of elliptic K3 surfaces with $E_{8}$ and $E_{7}$ fibers:

$$
y^{2}=x^{3}+x t^{3}\left(a_{0}+a_{1} t\right)+t^{5}\left(b_{0}+b_{1} t+b_{2} t^{2}\right) .
$$

To have discriminant -5 for the Picard group, the elliptic K3 surfaces in this family must have a section of height $\frac{5}{2}=4-\frac{3}{2}$. The $x$-coordinate for such a section must have the form $t^{2}\left(x_{0}+x_{1} t+h^{2} t^{2}\right)$. Substituting $x$ into the right-hand side and completing the square, we may solve for $b_{0}, b_{1}, b_{2}$ and $a_{1}$ in terms of $a_{0}, h, x_{0}$ and $x_{1}$. We then set $x_{1}=e h$ and $x_{0}=g+e^{2} / 4$ to simplify the expressions. Finally, we note that scaling $x, y, t$ by $\lambda^{2}, \lambda^{3}, \mu / \lambda$ gives $a_{0}, e, g, h$ weights $(1,3),(0,1)$, $(0,2)$ and $(-1,2)$ respectively with respect to $(\lambda, \mu)$. Therefore, we may scale $a_{0}$ and $e$ to equal 1 independently (at most removing hypersurfaces in the moduli space), and get the parametrization in the statement of the proposition. We note that the section $P$ of height $\frac{5}{2}$ is given by

$$
x(P)=\frac{1}{4} t^{2}\left((1+2 h t)^{2}+4 g\right), \quad y(P)=\frac{1}{8} t^{3}(1+2 h t)\left((1+2 h t)^{2}+6 g\right) .
$$


Corollary 15. The Humbert surface $\mathcal{H}_{5}$ is birational to $\mathbb{P}_{g, h}^{2}$, with the map to $\mathcal{A}_{2}$ given by the Igusa-Clebsch invariants

$$
\left(I_{2}: I_{4}: I_{6}: I_{10}\right)=\left(6(4 g+1), 9 g^{2}, 9\left(4 h+9 g^{3}+2 g^{2}\right), 4 h^{2}\right) .
$$

Proof. This follows immediately from Theorem 9. The Igusa-Clebsch invariants may be read out directly from the Weierstrass equation above.

Theorem 16. A birational model over $\mathbb{Q}$ of the Hilbert modular surface $Y_{-}(5)$ is given by the following double cover of $\mathbb{P}_{g, h}^{2}$ :

$$
z^{2}=2\left(6250 h^{2}-4500 g^{2} h-1350 g h-108 h-972 g^{5}-324 g^{4}-27 g^{3}\right) .
$$

It is a rational surface (i.e., birational to $\mathbb{P}^{2}$ ).

Proof. We follow the method of Section 4. The possible factors of the branch locus are $g, h, 8 h-9 g^{2}$ (the zero locus of this polynomial defines a subvariety of the moduli space for which the corresponding elliptic K3 surfaces acquire an extra $\mathrm{I}_{2}$ fiber), $64 h^{2}+48 g^{2} h+48 g^{5}+9 g^{4}$ (extra II fiber), and $6250 h^{2}-4500 g^{2} h-$ $1350 g h-108 h-972 g^{5}-324 g^{4}-27 g^{3}$ (extra $\mathrm{I}_{2}$ fiber). By Step 4 of the method, we deduce that only the last factor occurs, and the correct quadratic twist is by 2 . It was already well-known that the Hilbert modular surface is a rational surface, but we give an explicit parametrization in the following analysis.

6.2. Analysis. This is a rational surface: to obtain a parametrization, we complete the square in $h$, writing $h=k+9\left(250 g^{2}+75 g+6\right) / 6250$. Following this up with $k=3 m(10 g+3)(15 g+2) / 6250$, and removing square factors by writing $z=3 n(10 g+3)(15 g+2) / 25$, we obtain the equation $5 n^{2}-m^{2}+9+30 g=0$, which we can solve for $g$. This gives an explicit birational map between $Y_{-}(5)$ and $\mathbb{P}_{m, n}^{2}$.

The branch locus is the curve obtained by setting $z=0$, or alternatively $n=0$ in the above parametrization. It is parametrized by one variable $m$; we have

$$
(g, h)=\left(\frac{\left(m^{2}-9\right)}{30}, \frac{(m-2)^{2}(m+3)^{3}}{12500}\right) .
$$

6.3. Examples. We list in Table 1 some points of small height and corresponding genus- 2 curves. The second entry in the table is the modular curve $X_{0}(67) /\langle w\rangle$, where $w$ is the Atkin-Lehner involution. We find several other modular curves with real multiplication by $\mathcal{O}_{5}$ (and also a few for discriminants other than 5) corresponding to points of larger height.

6.4. Brumer's and Wilson's families of genus-2 curves. Genus- 2 curves whose Jacobians have real multiplication by $\mathcal{O}_{5}$ have been studied by Brumer [1995] and Wilson [1998; 2000]. Brumer describes a 3-dimensional family of genus-2 curves 


\begin{tabular}{l|c} 
Rational point $(g, h)$ & Sextic polynomial $f_{6}(x)$ defining the genus-2 curve $y^{2}=f_{6}(x)$ \\
\hline$\left(-\frac{8}{3}, \frac{47}{2}\right)$ & $-x^{5}+x^{4}-x^{3}-x^{2}+2 x-1$ \\
$\left(-\frac{37}{6}, 67\right)$ & $x^{6}+2 x^{5}+x^{4}-2 x^{3}+2 x^{2}-4 x+1$ \\
$\left(0, \frac{27}{50}\right)$ & $3 x^{5}+5 x^{3}+1$ \\
$\left(-\frac{1}{24}, \frac{1}{100}\right)$ & $4 x^{6}+4 x^{5}+5 x^{4}-5 x^{2}-2 x-2$ \\
$\left(-\frac{25}{54}, \frac{59}{81}\right)$ & $-x^{6}-2 x^{4}-6 x^{3}-5 x^{2}-6 x-1$ \\
$\left(-\frac{2}{3},-\frac{14}{25}\right)$ & $-7 x^{6}-7 x^{5}-5 x^{4}+5 x^{2}-x-1$ \\
$\left(\frac{47}{54}, \frac{71}{81}\right)$ & $3 x^{6}-6 x^{5}+7 x^{4}-2 x^{3}-2 x^{2}-1$ \\
$\left(-\frac{1}{6}, \frac{1}{25}\right)$ & $x^{6}-4 x^{5}+10 x^{4}-10 x^{3}+5 x^{2}+2 x-3$ \\
$\left(-\frac{4}{3}, \frac{16}{25}\right)$ & $-x^{6}-x^{5}+5 x^{2}-7 x-12$ \\
$\left(-\frac{4}{3}, \frac{49}{22}\right)$ & $7 x^{5}+5 x^{4}+3 x^{3}-9 x^{2}-14 x-7$ \\
$\left(0,-\frac{54}{11}\right)$ & $2 x^{6}-6 x^{5}-6 x^{4}+3 x^{3}-18 x^{2}-6 x-2$ \\
$\left(\frac{11}{6}, \frac{53}{11}\right)$ & $9 x^{6}-14 x^{5}+13 x^{4}-2 x^{3}-22 x^{2}-8 x-7$ \\
$\left(-\frac{5}{24}, \frac{1}{64}\right)$ & $6 x^{6}-2 x^{5}-15 x^{4}-16 x^{3}-25 x^{2}-8 x-4$ \\
$\left(\frac{11}{6},-\frac{89}{25}\right)$ & $-x^{6}+2 x^{5}-5 x^{4}+30 x^{3}-10 x^{2}+8 x-1$ \\
$\left(-\frac{2}{3}, \frac{68}{11}\right)$ & $-2 x^{6}-2 x^{5}-11 x^{4}-29 x^{3}-31 x^{2}-26 x-6$ \\
$\left(-\frac{2}{3}, \frac{26}{25}\right)$ & $-2 x^{6}+36 x^{5}+5 x^{4}+35 x^{3}-10 x^{2}-21 x-17$ \\
\hline
\end{tabular}

Table 1. Some points of height $\leq 100$ on the surface of Theorem 16 and the corresponding genus- 2 curves.

given by

$$
\begin{aligned}
y^{2}+\left(1+x+x^{3}\right. & \left.+c\left(x+x^{2}\right)\right) y \\
& =-b d x^{4}+(b-d-2 b d) x^{3}+(1-3 b-b d) x^{2}+(1+3 b) x+b .
\end{aligned}
$$

In the online supplement, we give formulas for the corresponding values of our parameters $g$ and $h$.

Wilson describes a family of genus- 2 curves by their Igusa-Clebsch invariants. His moduli space is 2-dimensional, though he uses three coordinates $z_{6}, s_{2}$ and $\sigma_{5}$ of weights 1,2, 5 respectively, in a weighted projective space. These coordinates are related to ours via

$$
(g, h)=\left(-\frac{2 z_{6}^{2}+s_{2}}{12 z_{6}^{2}}, \frac{\sigma_{5}}{64 z_{6}^{5}}\right) .
$$

\section{Discriminant 8}

7.1. Parametrization. We start with an elliptic K3 surface with fibers of type $D_{9}$ and $E_{7}$. A Weierstrass equation for such a family is given by

$$
y^{2}=x^{3}+t((2 r+1) t+r) x^{2}+2 r s t^{4}(t+1) x+r s^{2} t^{7} .
$$


We then identify a fiber of type $E_{8}$, and transform to it by a 2-neighbor step.

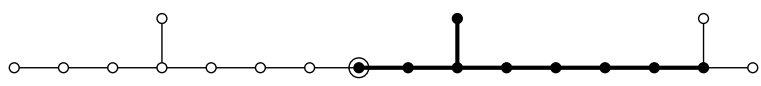

The resulting elliptic fibration has $E_{8}$ and $E_{7}$ fibers, and we may read out the Igusa-Clebsch invariants, and then compute the branch locus of the double cover that defines the Hilbert modular surface. It corresponds to elliptic K3 surfaces with an extra $\mathrm{I}_{2}$ fiber. We obtain the following result for $Y_{-}(8)$.

Theorem 17. The Humbert surface $\mathcal{H}_{8}$ is birational to $\mathbb{P}_{r, s}^{2}$, with the explicit map to $\mathcal{A}_{2}$ given by the Igusa-Clebsch invariants

$$
\begin{aligned}
I_{2} & =-4(3 s+8 r-2), \\
I_{4} & =4\left(9 r s+4 r^{2}+4 r+1\right), \\
I_{6} & =-4\left(36 r s^{2}+94 r^{2} s-35 r s+4 s+48 r^{3}+40 r^{2}+4 r-2\right), \\
I_{10} & =-8 s^{2} r^{3} .
\end{aligned}
$$

A birational model over $\mathbb{Q}$ for the Hilbert modular surface $Y_{-}(8)$ as a double cover

Rational point $(r, s) \quad$ Sextic polynomial $f_{6}(x)$ defining the genus-2 curve $y^{2}=f_{6}(x)$

\begin{tabular}{l|c}
\hline$\left(\frac{2}{25}, \frac{83}{50}\right)$ & $x^{6}-x^{5}+3 x^{4}+x^{3}+x^{2}+2 x+1$ \\
$\left(-\frac{34}{9}, \frac{50}{9}\right)$ & $x^{6}-2 x^{5}-2 x^{4}-4 x^{3}+2 x^{2}+4 x-3$ \\
$\left(-22, \frac{59}{2}\right)$ & $x^{6}+2 x^{5}-3 x^{4}+5 x^{3}+x^{2}-x-1$ \\
$\left(\frac{2}{49}, \frac{58}{49}\right)$ & $-x^{6}-4 x^{5}-6 x^{4}+2 x^{2}+2 x-1$ \\
$\left(-52, \frac{83}{2}\right)$ & $-4 x^{6}+2 x^{5}+5 x^{4}-7 x^{3}-x+1$ \\
$\left(\frac{1}{8}, \frac{59}{32}\right)$ & $-x^{6}-x^{5}-7 x^{2}+5 x-4$ \\
$\left(-\frac{4}{9}, \frac{23}{8}\right)$ & $-2 x^{6}+6 x^{5}-x^{4}-7 x^{3}-x^{2}-3 x$ \\
$\left(80, \frac{83}{2}\right)$ & $x^{6}+5 x^{5}+8 x^{4}+5 x^{3}+5 x^{2}-8 x+4$ \\
$\left(\frac{19}{2}, 22\right)$ & $-x^{6}-4 x^{5}-8 x^{4}-8 x^{3}-8 x^{2}+4 x-4$ \\
$(94,-54)$ & $-x^{6}+6 x^{4}-8 x^{3}+6 x^{2}+6 x-9$ \\
$\left(\frac{13}{8},-\frac{2}{9}\right)$ & $-x^{6}+6 x^{5}-9 x^{4}+3 x^{2}-6 x-2$ \\
$\left(\frac{86}{9},-\frac{13}{2}\right)$ & $x^{6}-7 x^{4}-7 x^{3}-x^{2}+9 x+9$ \\
$\left(-\frac{3}{8}, \frac{9}{4}\right)$ & $-3 x^{6}-9 x^{5}-2 x^{4}+10 x^{3}+x^{2}+3 x$ \\
$\left(\frac{1}{14}, \frac{10}{7}\right)$ & $-x^{6}-2 x^{5}-7 x^{4}+4 x^{3}-3 x^{2}+10 x-5$ \\
$\left(\frac{1}{18}, \frac{31}{18}\right)$ & $-3 x^{6}-10 x^{5}-7 x^{4}-5 x^{2}+2 x-1$ \\
$\left(\frac{1}{32}, \frac{19}{16}\right)$ & $4 x^{6}-7 x^{5}-3 x^{4}-2 x^{3}+10 x^{2}+5 x+1$ \\
\hline
\end{tabular}

Table 2. Some points of small height on the surface of Theorem 17 and the corresponding genus- 2 curves. 
of $\mathbb{P}^{2}$ is given by the equation

$$
z^{2}=2\left(16 r s^{2}+32 r^{2} s-40 r s-s+16 r^{3}+24 r^{2}+12 r+2\right) .
$$

It is also a rational surface.

7.2. Analysis. This Hilbert modular surface is a rational surface. To see this, we may complete the square in $s$, setting $s=s_{1}-r+5 / 4+1 /(32 r)$. Then setting $s_{1}=m(16 r-1) /(32 r)$ and $z=n(16 r-1)$, we remove square factors from the equation, which becomes linear in $r$. We find $r=\left(m^{2}-1\right) /\left(32 n^{2}-16\right)$, thus obtaining an explicit parametrization of $Y_{-}(8)$ by $\mathbb{P}_{m, n}^{2}$.

The branch locus is a genus- 0 curve; we obtain a parametrization by setting $z=0$ :

$$
(r, s)=\left(\frac{1-t^{2}}{16}, \frac{(t+3)^{3}}{16(t+1)}\right)
$$

7.3. Examples. Table 2 lists some points of small height and their genus- 2 curves.

\section{Discriminant 12}

8.1. Parametrization. We start with an elliptic $\mathrm{K} 3$ surface with fibers of types $E_{8}$, $D_{6}$ and $A_{2}$. The Weierstrass equation for such a family is given by

$$
y^{2}=x^{3}+\left(\left(1-f^{2}\right)(1-t)+t\right) t x^{2}+2 e t^{3}(t-1) x+e^{2}(t-1)^{2} t^{5} .
$$

We identify a fiber of type $E_{7}$, and move to the associated elliptic fibration by a 2-neighbor step.

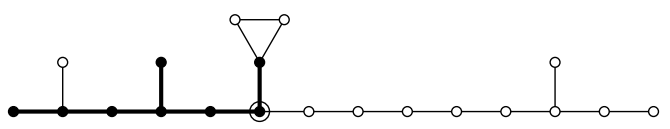

The new elliptic fibration has $E_{8}$ and $E_{7}$ fibers, and so we may read out the Igusa-Clebsch invariants, and determine the branch locus for the Hilbert modular surface.

Theorem 18. A birational model over $\mathbb{Q}$ for the Hilbert modular surface $Y_{-}(12)$ as a double cover of $\mathbb{P}_{e, f}^{2}$ is given by the equation

$$
z^{2}=(f-1)(f+1)\left(f^{6}-f^{4}-18 e f^{2}+27 e^{2}+16 e\right) .
$$

It is a rational surface.

8.2. Analysis. Note the extra involution $(e, f) \mapsto(e,-f)$ arising from the HurwitzMaass extension (as described at the end of Section 3), since 12 is not a prime power. In fact, there are two independent involutions evident in the diagram above, but one of them has been used up to fix the Weierstrass scaling of $x$ (namely, the coefficient of $x^{2}$ evaluates to 1 at $t=1$ ). The other involution is reflected in the 
Weierstrass equation for the universal family of elliptic K3 surfaces as $f \mapsto-f$. It preserves the branch locus of the map $Y_{-}(12) \rightarrow \mathcal{H}_{12}$, and therefore lifts to $Y_{-}$(12).

The branch locus has three components; the two simple components $f= \pm 1$ correspond to the $D_{6}$ fiber getting promoted to an $E_{7}$ fiber, while the remaining component corresponds to having an extra $\mathrm{I}_{2}$ fiber. This last component is a rational curve; completing the square with respect to $e$, we find after some easy algebraic manipulation the parametrization

$$
(e, f)=\left(\frac{16\left(h^{2}-1\right)}{\left(h^{2}+3\right)^{3}}, \frac{-4 h}{h^{2}+3}\right) .
$$

This Hilbert modular surface is rational as well. To obtain an explicit parametrization, note that the right-hand side of the above equation is quadratic in $e$, and $e=0$ makes it a square. Therefore the conic bundle over $\mathbb{P}_{f}^{1}$ has a section. Setting $z=g e+f^{2}\left(f^{2}-1\right)$, we may solve for $e$, obtaining a birational parametrization by $\mathbb{P}_{f, g}^{2}$.

We will not list the Igusa-Clebsch invariants for this (and higher) discriminants, as they are complicated expressions. They are available in the online supplement.

8.3. Examples. Table 3 lists some points of small height and their genus- 2 curves.

Rational point $(e, f) \quad$ Sextic polynomial $f_{6}(x)$ defining the genus-2 curve $y^{2}=f_{6}(x)$

\begin{tabular}{l|c}
\hline$\left(\frac{34}{27}, \frac{5}{3}\right)$ & $-2 x^{6}-2 x^{5}+x^{4}-3 x^{2}+2 x-1$ \\
$\left(\frac{34}{27},-\frac{5}{3}\right)$ & $x^{5}-x^{4}+x^{3}-3 x^{2}-x+5$ \\
$\left(\frac{51}{100}, 2\right)$ & $-3 x^{6}+6 x^{5}+4 x^{4}-2 x^{3}-8 x^{2}-6 x-6$ \\
$\left(-\frac{11}{3},-2\right)$ & $-x^{6}-8 x^{3}+12 x-12$ \\
$\left(-\frac{11}{3}, 2\right)$ & $-x^{6}+12 x^{4}+8 x^{3}-12 x^{2}+12 x-4$ \\
$\left(\frac{4}{3},-2\right)$ & $-8 x^{6}+12 x^{4}+8 x^{3}-6 x^{2}-12 x-3$ \\
$\left(\frac{4}{3}, 2\right)$ & $-5 x^{6}+12 x^{5}-6 x^{4}+8 x^{3}-12 x^{2}-8$ \\
$\left(-\frac{14}{27}, \frac{1}{3}\right)$ & $-x^{5}+13 x^{4}-6 x^{3}-2 x^{2}+3 x-7$ \\
$\left(-\frac{29}{14},-\frac{15}{14}\right)$ & $x^{6}+2 x^{5}+13 x^{4}-16 x^{3}+17 x^{2}+4 x+8$ \\
$\left(\frac{80}{81}, 2\right)$ & $-8 x^{6}-12 x^{5}+15 x^{4}+5 x^{3}-21 x^{2}+15 x-5$ \\
$\left(\frac{51}{100},-2\right)$ & $-x^{6}-6 x^{5}-11 x^{4}-14 x^{3}-23 x^{2}+6 x+3$ \\
$\left(-\frac{5}{2},-\frac{3}{2}\right)$ & $-5 x^{6}-10 x^{5}-x^{4}+24 x^{3}-5 x^{2}-8 x-20$ \\
$\left(-\frac{23}{54},-\frac{1}{2}\right)$ & $x^{6}-6 x^{5}-3 x^{4}+24 x^{3}-3 x^{2}-4$ \\
$\left(\frac{25}{18},-\frac{3}{2}\right)$ & $4 x^{6}-24 x^{5}+27 x^{4}-28 x^{3}+21 x^{2}-5$ \\
$\left(-\frac{5}{54}, \frac{2}{3}\right)$ & $3 x^{6}-26 x^{5}+31 x^{4}+12 x^{3}-3 x^{2}-10 x-15$ \\
$\left(\frac{13}{64},-\frac{5}{4}\right)$ & $-5 x^{6}+3 x^{5}-12 x^{4}+28 x^{3}+12 x^{2}-36 x$ \\
\hline
\end{tabular}

Table 3. Some points of small height on the surface of Theorem 18 and the corresponding genus- 2 curves. 
We mention a few special curves on the Hilbert modular surface, which one may recognize by looking at a plot of the rational points. First, specializing $f$ to some $f_{0} \in \mathbb{Q}$ gives a conic in $z$ and $e$, which always has a rational point (since, as we noted above, $e=0$ makes the right-hand side a square). These may be used to produce many rational points and examples of genus- 2 curves when the Brauer obstruction happens to vanish.

Another noticeable feature of the plot is the parabola $e=4\left(f^{2}-1\right) / 9$. We check that the Brauer obstruction vanishes identically on this locus, giving a family of genus-2 curves whose Jacobians have real multiplication by $\mathcal{O}_{12}$. Note that this is a non-modular curve: generically the endomorphism ring is no larger (i.e., not a quaternion algebra).

\section{Discriminant 13}

9.1. Parametrization. We start with an elliptic K3 surface with reducible fibers of types $E_{8}, E_{6}$ and $A_{1}$, and with a section of height $\frac{13}{6}=4-\frac{4}{3}-\frac{1}{2}$. A Weierstrass equation for such a family is given by

$$
y^{2}=x^{3}+(4 g+1) t^{2} x^{2}-4 g(h-g-1)(t-1) t^{3} x+4 g^{2}(t-1)^{2} t^{4}\left(h^{2} t+1\right) .
$$

We identify an $E_{7}$ fiber below and move to it by a 2-neighbor step.

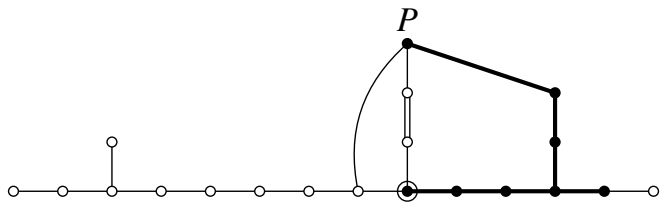

The resulting elliptic fibration has $E_{8}$ and $E_{7}$ fibers, so we may read out the Igusa-Clebsch invariants and work out the branch locus of the double cover defining the Hilbert modular surface. It corresponds to the elliptic K3 surface having an extra $\mathrm{I}_{2}$ fiber.

Theorem 19. A birational model over $\mathbb{Q}$ for the Hilbert modular surface $Y_{-}(13)$ as a double cover of $\mathbb{P}_{g, h}^{2}$ is given by the equation

$$
z^{2}=108 g h^{3}-\left(27 g^{2}+468 g-4\right) h^{2}+8\left(82 g^{2}+71 g-2\right) h-16(2 g-1)^{3} .
$$

It is a rational surface.

9.2. Analysis. The surface $Y_{-}(13)$ is rational. To show this, we proceed as follows. The substitutions $g=g_{1}-\frac{1}{54}$ and $h=h_{1}+\frac{64}{27}$, followed by $h_{1}=m g_{1}$, make the right-hand side quadratic in $g_{1}$, up to a square factor. Removing this factor by setting $z=z_{1} g_{1} / 18$, and then setting $g_{1}=g_{2} / 54$, we get the conic bundle over $\mathbb{Q}(m)$

$z_{1}^{2}=3 m^{2}(4 m-1) g_{2}^{2}-6\left(2 m^{3}-301 m^{2}-528 m+128\right) g_{2}-507\left(m^{2}-96 m-1024\right)$. 
Furthermore, $g_{2}=1$ makes the resulting expression a square, giving us a rational point on the conic over $\mathbb{Q}(m)$. Therefore, we may set $z_{1}=36(m+20)+n\left(g_{2}-1\right)$ and solve for $g_{2}$. This gives us a birational parametrization of $Y_{-}(13)$ by $\mathbb{P}_{m, n}^{2}$.

We can also deduce that the branch locus is a rational curve, as follows. By setting $z_{1}=0$, we obtain a quadratic equation in $g_{2}$ whose discriminant, up to a square factor, is $m^{2}-44 m+16$. Setting it equal to $n^{2}$ and noting that $m=0$ makes the expression a square, we obtain a parametrization of this conic, as

$$
m=-4 \frac{2 r+11}{r^{2}-1}
$$

Working backwards, we obtain a parametrization of the branch locus as

$$
(g, h)=\left(\frac{-2(r-2)^{2}(r+1)}{27(r+7)}, \frac{2(2 r+5)^{3}}{27(r+1)(r+7)}\right) .
$$

9.3. Examples. Table 4 lists some points of small height and their genus- 2 curves.

On a plot of rational points we observe the line $h=(g+4) / 3$, along which the Brauer obstruction vanishes, leading to a family of genus- 2 curves whose Jacobians have "honest" real multiplication by $\mathcal{O}_{13}$.

Rational point $(g, h) \quad$ Sextic polynomial $f_{6}(x)$ defining the genus-2 curve $y^{2}=f_{6}(x)$

\begin{tabular}{lc}
\hline$\left(-17, \frac{1}{3}\right)$ & $-3 x^{6}-6 x^{5}+4 x^{3}+3 x^{2}+6 x-5$ \\
$\left(-\frac{13}{2},-\frac{11}{2}\right)$ & $x^{5}+5 x^{4}+5 x^{3}-5 x^{2}+6 x-1$ \\
$\left(-\frac{17}{2}, \frac{7}{2}\right)$ & $-x^{5}-2 x^{4}-3 x^{3}-6 x^{2}-7$ \\
$\left(\frac{11}{5}, \frac{1}{5}\right)$ & $x^{6}+4 x^{5}+2 x^{4}-8 x^{3}-5 x^{2}-5$ \\
$\left(-\frac{1}{3}, \frac{11}{9}\right)$ & $-x^{6}+3 x^{4}+12 x^{3}+6 x^{2}-11$ \\
$\left(-\frac{14}{11},-\frac{2}{11}\right)$ & $13 x^{6}+12 x^{5}+6 x^{4}+10 x^{3}-7 x^{2}-2 x+1$ \\
$\left(-\frac{2}{17}, \frac{2}{17}\right)$ & $-x^{6}-2 x^{5}-x^{4}+14 x^{3}+2 x^{2}-8 x-9$ \\
$\left(\frac{1}{5}, \frac{17}{15}\right)$ & $3 x^{6}+12 x^{5}+6 x^{4}-4 x^{3}-15 x^{2}+5$ \\
$\left(-\frac{10}{3},-\frac{10}{9}\right)$ & $-x^{6}+18 x^{5}+3 x^{4}-6 x^{3}+6 x^{2}-5$ \\
$(-10,6)$ & $-x^{6}-6 x^{4}-10 x^{3}-9 x^{2}-30 x+11$ \\
$\left(-\frac{18}{5}, \frac{14}{5}\right)$ & $-x^{6}-2 x^{4}-10 x^{3}+7 x^{2}-30 x-9$ \\
$\left(-\frac{7}{13}, \frac{3}{13}\right)$ & $-31 x^{6}+12 x^{5}-30 x^{4}+4 x^{3}-33 x^{2}-12 x-1$ \\
$\left(\frac{10}{3}, \frac{10}{3}\right)$ & $7 x^{6}+18 x^{5}-9 x^{4}-34 x^{3}+18 x^{2}-5$ \\
$\left(-11,-\frac{11}{9}\right)$ & $-5 x^{6}+6 x^{5}+3 x^{4}-4 x^{3}+18 x^{2}-36 x-9$ \\
$\left(-\frac{7}{8}, \frac{10}{3}\right)$ & $-x^{6}+3 x^{4}-20 x^{3}+30 x^{2}-36 x+9$ \\
$\left(-\frac{13}{9}, \frac{13}{9}\right)$ & $-x^{6}-9 x^{4}+8 x^{3}-30 x^{2}+36 x-3$ \\
\hline
\end{tabular}

Table 4. Some points of small height on the surface of Theorem 19 and the corresponding genus- 2 curves. 


\section{Discriminant 17}

10.1. Parametrization. We start with an elliptic K3 surface with a $I_{17}$ fiber. A Weierstrass equation for such a family of surfaces is given by

$$
\begin{aligned}
& y^{2}=x^{3} \\
& \quad+\left(1+2 g t+\left(2 h+(g+1)^{2}\right) t^{2}+2\left(g h+g+2 g^{2}+h\right) t^{3}+\left((g+h)^{2}+2 g^{3}\right) t^{4}\right) x^{2} \\
& \quad-4 h^{2} t^{5}\left(1+g t+(h+2 g+1) t^{2}+\left(h+2 g^{2}+g\right) t^{3}\right) x+4 h^{4} t^{10}\left((2 g+1) t^{2}+1\right) .
\end{aligned}
$$

We first identify an $E_{7}$ fiber and go to the associated elliptic fibration via a 2-neighbor step.

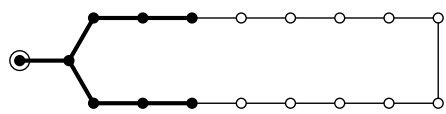

The resulting elliptic fibration has $E_{7}$ and $A_{8}$ fibers, and a section $P$ of height $\frac{17}{18}=4-\frac{3}{2}-\frac{14}{9}$. We next identify a fiber $F^{\prime}$ of type $E_{8}$ and perform a 3-neighbor step to move to the associated elliptic fibration.

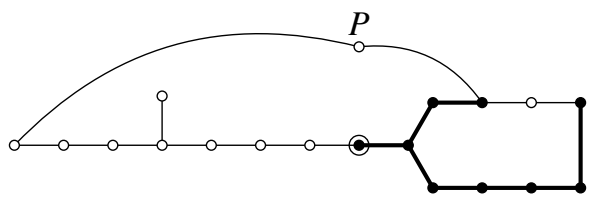

Since $P \cdot F^{\prime}=2$, while the remaining component of the $A_{8}$ fiber intersects $F^{\prime}$ with multiplicity 3 , the new elliptic fibration has a section. We may therefore convert to the Jacobian; this has $E_{8}$ and $E_{7}$ fibers, and we may read out the Igusa-Clebsch invariants.

Theorem 20. A birational model over $\mathbb{Q}$ for the Hilbert modular surface $Y_{-}(17)$ as a double cover of $\mathbb{P}^{2}$ is given by the equation

$$
\begin{aligned}
z^{2}=-256 h^{3}+\left(192 g^{2}+\right. & 464 g+185) h^{2} \\
- & 2(2 g+1)\left(12 g^{3}-65 g^{2}-54 g-9\right) h+(g+1)^{4}(2 g+1)^{2} .
\end{aligned}
$$

It is a rational surface.

10.2. Analysis. This is evidently a rational elliptic surface over $\mathbb{P}_{g}^{1}$. In fact, it is a rational surface over $\mathbb{Q}$. We exhibit a birational parametrization as follows: set $h=(4(2 g+1)+m(2 g+1)(27 g+13)) / 27$ and absorb square factors by setting $z=z_{1}(2 g+1)(27 g+13) / 243$, to get (after scaling $\left.g=g_{1} / 3\right)$ the equation $z_{1}^{2}=-9(8 m-1)^{3} g_{1}^{2}-2(16 m+7)\left(424 m^{2}-385 m+1\right) g_{1}$

$$
-3\left(3328 m^{3}-1923 m^{2}-3138 m-803\right)
$$

which is a conic bundle over $\mathbb{P}_{m}^{1}$. We see that $g_{1}=-\frac{3}{2}$ makes the right hand side a 
square, and so setting $z_{1}=9(2 m+11) / 2+n\left(g_{1}+\frac{3}{2}\right)$ and solving for $g_{1}$, we get a birational map from $\mathbb{P}_{m, n}^{2}$.

The branch locus is a curve of genus 0 . To produce a parametrization, we set $z_{1}=0$ and note that the discriminant of the resulting quadratic equation in $g_{1}$ is a square times $64 m^{2}+218 m-8$. Setting $64 m^{2}+218 m-8=(8 m+r)^{2}$ and solving for $m$, we ultimately obtain:

$$
(g, h)=\left(\frac{-\left(8 r^{3}-111 r^{2}+1212 r-8146\right)}{2(2 r-7)^{3}}, \frac{(r-17)^{2}(r+10)^{4}}{4(2 r-7)^{6}}\right) .
$$

10.3. Examples. Table 5 lists some points of small height and their genus- 2 curves.

A plot of the rational points on $Y_{-}(17)$ reveals two special curves on the surface. First, there is the line $h=-g / 2$. Substituting this into the equation for the Hilbert modular surface, we obtain a conic, which can be parametrized as

$$
(g, h)=\left(-\frac{m^{2}-4}{2(m-6)}, \frac{m^{2}-4}{4(m-6)}\right) .
$$

The Brauer obstruction vanishes along this locus. However, this curve is modular: the endomorphism ring is a split quaternion algebra, strictly containing $\mathcal{O}_{17}$. The

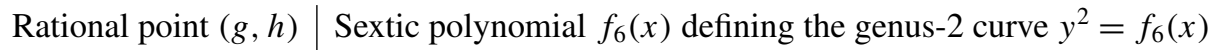

\begin{tabular}{l|c}
\hline$\left(0, \frac{13}{32}\right)$ & $-2 x^{6}-x^{5}-6 x^{4}-5 x^{3}-4 x^{2}-4 x$ \\
$\left(-\frac{5}{11}, \frac{1}{88}\right)$ & $3 x^{6}+4 x^{5}+4 x^{4}-6 x^{3}-5 x^{2}-4 x+4$ \\
$(6,26)$ & $-2 x^{6}+2 x^{5}+x^{4}+8 x^{3}+7 x^{2}+4 x$ \\
$\left(\frac{9}{4}, \frac{77}{64}\right)$ & $-2 x^{6}-x^{5}+8 x^{4}-5 x^{3}-4 x^{2}+4 x-4$ \\
$\left(\frac{3}{5},-\frac{11}{50}\right)$ & $-8 x^{6}+8 x^{5}+7 x^{4}+2 x^{3}-x$ \\
$(5,-11)$ & $4 x^{5}+9 x^{4}+2 x^{3}-8 x^{2}-2 x$ \\
$(5,22)$ & $2 x^{6}-4 x^{5}+9 x^{4}-10 x^{3}+4 x^{2}-4 x$ \\
$\left(\frac{1}{5}, \frac{28}{25}\right)$ & $-10 x^{6}-10 x^{5}-2 x^{4}-7 x^{3}-x$ \\
$\left(-\frac{1}{4},-\frac{11}{64}\right)$ & $4 x^{5}+3 x^{4}+11 x^{3}-7 x^{2}+x$ \\
$\left(\frac{5}{4},-\frac{35}{64}\right)$ & $x^{5}-7 x^{3}+2 x^{2}-8 x+12$ \\
$\left(-\frac{5}{2},-\frac{13}{8}\right)$ & $4 x^{5}-x^{4}-13 x^{3}-3 x^{2}+13 x$ \\
$\left(\frac{1}{5},-\frac{7}{20}\right)$ & $3 x^{6}+7 x^{5}+6 x^{4}+16 x^{3}+14 x^{2}-8 x-8$ \\
$\left(-12,-\frac{23}{2}\right)$ & $-7 x^{6}-19 x^{5}-7 x^{4}+14 x^{3}-x$ \\
$\left(0,-\frac{1}{16}\right)$ & $-4 x^{6}+19 x^{5}-20 x^{4}+11 x^{3}+15 x^{2}-8 x-4$ \\
$\left(2,-\frac{35}{4}\right)$ & $-4 x^{5}+20 x^{4}-12 x^{3}-15 x^{2}-4 x$ \\
$\left(4, \frac{51}{8}\right)$ & $-4 x^{6}-12 x^{5}-27 x^{4}+2 x^{3}+12 x^{2}+18 x$ \\
\hline
\end{tabular}

Table 5. Some points of small height on the surface of Theorem 20 and the corresponding genus- 2 curves. 
second curve is the parabola $h=-\left(6 g^{2}+g-1\right) / 8$. The Brauer obstruction vanishes along this curve too, giving a 1-parameter family of genus-2 curves whose Jacobians have real multiplication by $\mathcal{O}_{17}$.

\section{Discriminant 21}

11.1. Parametrization. We start with an elliptic $\mathrm{K} 3$ surface with fibers of type $E_{8}$, $A_{6}$ and $A_{2}$ at $t=\infty, 0$ and 1 respectively.

A Weierstrass equation for such a family is

$$
y^{2}=x^{3}+\left(a_{0}+a_{1} t+a_{2} t^{2}\right) x^{2}+2 t^{2}(t-1)\left(b_{0}+b_{1} t\right) x+t^{4}(t-1)^{2}\left(c_{0}+c_{1} t\right)
$$

with

$$
\begin{array}{lll}
a_{0}=1, & a_{1}=-r^{2}+2 r s-1, & a_{2}=(r-s)^{2} ; \\
b_{0}=\left(r^{2}-1\right)(s-r)^{2}, & b_{1}=\left(r^{2}-1\right)(s-r)^{2}(r s-1) ; & \\
c_{0}=\left(r^{2}-1\right)^{2}(s-r)^{4}, & c_{1}=\left(r^{2}-1\right)^{3}(s-r)^{4} . &
\end{array}
$$

We identify a fiber of type $E_{7}$, and a 3-neighbor step gives us the desired $E_{8} E_{7}$ fibration.

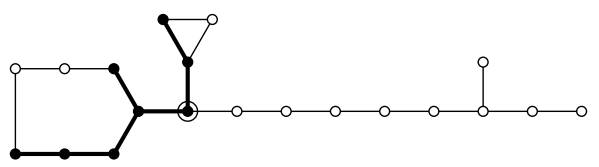

We read out the Igusa-Clebsch invariants, and the branch locus of $Y_{-}(21)$ as a double cover of $\mathbb{P}_{r, s}^{2}$ corresponds to the subvariety of the moduli space where the elliptic K3 surfaces have an extra $I_{2}$ fiber.

Theorem 21. A birational model over $\mathbb{Q}$ for the Hilbert modular surface $Y_{-}(21)$ as a double cover of $\mathbb{P}_{r, s}^{2}$ is given by the equation

$$
\begin{aligned}
z^{2}=16 s^{4}-8 r\left(27 r^{2}-23\right) s^{3}+ & \left(621 r^{4}-954 r^{2}+349\right) s^{2} \\
- & 18\left(r^{3}-r\right)\left(33 r^{2}-29\right) s+\left(r^{2}-1\right)\left(189\left(r^{4}-r^{2}\right)+16\right) .
\end{aligned}
$$

It is a singular $\mathrm{K} 3$ surface (i.e., of Picard number 20).

11.2. Analysis. The extra involution (corresponding to $21=3 \cdot 7$ ) here is given by $(r, s) \mapsto(-r,-s)$.

The branch locus is a rational curve; a parametrization is given by

$$
(r, s)=\left(\frac{h^{4}+72 h^{2}-81}{18 h\left(h^{2}+3\right)}, \frac{\left(h^{2}-9\right)\left(h^{4}-126 h^{2}+189\right)}{432 h\left(h^{2}+3\right)}\right) .
$$

The equation of $Y_{-}(21)$ above expresses it as a surface fibered by genus-1 curves over $\mathbb{P}_{r}^{1}$. In fact, since the coefficient of $s^{4}$ is a square, this fibration has a section, 
and we may convert it to its Jacobian form, which after some simple Weierstrass transformations may be written as

$$
\begin{aligned}
z^{2}=w^{3}+\left(-27 r^{4}+43\right) w^{2}+4\left(r^{2}-1\right)\left(8127 r^{4}-18459 r^{2}+9740\right) w \\
+4\left(r^{2}-1\right)^{2} w\left(-186624 r^{6}+1320813 r^{4}-1817964 r^{2}+705679\right) .
\end{aligned}
$$

This is a K3 surface with an elliptic fibration to $\mathbb{P}_{r}^{1}$. The discriminant of the cubic polynomial is

$$
\left(r^{2}-1\right)^{3}\left(27 r^{2}-25\right)^{2}\left(27 r^{4}+342 r^{2}-289\right)^{3},
$$

from which we deduce that we have three $\mathrm{I}_{2}$ fibers (including $r=\infty$ ) and six $\mathrm{I}_{3}$ fibers, which contribute 15 to the Picard number. We find the following sections.

$$
\begin{aligned}
& P_{0}=\left(6\left(r^{2}-1\right)\left(6 r^{2}-7\right), 4(r-1)(r+1)\left(27 r^{4}+342 r^{2}-289\right)\right), \\
& P_{1}=\left(2\left(324 r^{4}-1503 r^{2}+1019\right) / 21,8\left(27 r^{2}-25\right)\left(27 r^{4}+342 r^{2}-289\right) /(21 \mu)\right), \\
& P_{2}=\left((-102+32 v)\left(r^{2}-1\right), 32 v r\left(r^{2}-1\right)\left(-27 r^{2}+31+22 v\right)\right), \\
& P_{3}=\left((-102-32 v)\left(r^{2}-1\right),-32 v r\left(r^{2}-1\right)\left(-27 r^{2}+31-22 v\right)\right) .
\end{aligned}
$$

Here $\mu=\sqrt{21}$ and $v=\sqrt{-1}$. Note that $P_{0}$ is a 3-torsion section, whereas the height matrix for $P_{1}, P_{2}, P_{3}$ is

$$
\frac{1}{6}\left(\begin{array}{rrr}
2 & 0 & 0 \\
0 & 13 & 1 \\
0 & 1 & 13
\end{array}\right) .
$$

Therefore, the Picard number of the surface is 20, and the discriminant of the lattice spanned by these sections and the trivial lattice is $1008=2^{4} 3^{2} 7$. We showed that this is the entire Néron-Severi group by checking that the above subgroup of the Mordell-Weil group is 2- and 3-saturated.

Using $P_{0}$, we may rewrite the equation in the much simpler form

$$
z^{2}+\left(9 r^{2}-13\right) w z+\left(r^{2}-1\right)\left(27 r^{4}+342 r^{2}-289\right) z=w^{3} .
$$

The quotient of the Hilbert modular surface by the involution

$$
(r, s, z) \mapsto(-r,-s,-z)
$$

is the rational elliptic surface

$$
\begin{aligned}
z^{2}=w^{3}+\left(-27 t^{2}+\right. & 43) w^{2}+4(t-1)\left(8127 t^{2}-18459 t+9740\right) w \\
& +4(t-1)^{2}\left(-186624 t^{3}+1320813 t^{2}-1817964 t+705679\right) .
\end{aligned}
$$


It has three reducible fibers of type $\mathrm{I}_{3}$ and one of type $\mathrm{I}_{2}$, a 3-torsion section, and Mordell-Weil rank 1, generated by the following section of height $\frac{1}{6}$ :

$$
(z, w)=\left(2\left(324 r^{2}-1503 r+1019\right) / 21,8(27 r-25)\left(27 r^{2}+342 r-289\right) /(21 \mu)\right) .
$$

11.3. Examples. Table 6 lists some points of small height and their genus- 2 curves. The torsion section $P_{0}$ on the Jacobian model of $Y_{-}(21)$ pulls back to the curve

$$
s=\frac{45 r^{3}+9 r^{2}-45 r-17}{2\left(27 r^{2}+6 r-17\right)} .
$$

The Brauer obstruction always vanishes for this family, and yields a family of genus-2 curves over $\mathbb{Q}(r)$, whose Jacobians have real multiplication by $\mathcal{O}_{21}$.

Another special curve which we observe from a plot of the rational points is the hyperbola $s^{2}=\left(3 r^{2}+1\right) / 4$. It may be parametrized as

$$
(r, s)=\left(\frac{4 m}{4 m^{2}-3}, \frac{3+4 m^{2}}{2\left(3-4 m^{2}\right)}\right) \text {. }
$$

The Brauer obstruction also vanishes for this family.

Rational point $(r, s) \quad$ Sextic polynomial $f_{6}(x)$ defining the genus-2 curve $y^{2}=f_{6}(x)$

$\left(\frac{3}{2}, \frac{1}{44}\right)$

$\left(\frac{21}{34},-\frac{43}{68}\right)$

$\left(-\frac{36}{13},-\frac{49}{26}\right)$

$\left(0, \frac{1}{2}\right)$

$\left(0,-\frac{1}{2}\right)$

$\left(\frac{45}{46},-\frac{25}{92}\right)$

$\left(-\frac{3}{2},-\frac{1}{44}\right)$

$\left(-\frac{21}{34}, \frac{43}{68}\right)$

$\left(-\frac{45}{46}, \frac{25}{92}\right)$

$\left(3,-\frac{1}{28}\right)$

$\left(\frac{55}{63},-\frac{4}{63}\right)$

$\left(-\frac{1}{3},-\frac{11}{9}\right)$

$\left(-3, \frac{1}{28}\right)$

$\left(\frac{1}{9}, \frac{11}{9}\right)$

$\left(-4,-\frac{7}{2}\right)$

$\left(-\frac{35}{69}, \frac{11}{69}\right)$

$$
\begin{gathered}
-5 x^{6}-8 x^{5}+20 x^{4}+5 x^{3}-13 x \\
13 x^{6}+26 x^{5}+33 x^{4}+9 x^{3}-5 x^{2}-11 x \\
7 x^{6}-42 x^{5}-44 x^{4}-12 x^{3}-11 x^{2}-14 x-7 \\
13 x^{6}+54 x^{5}+32 x^{4}-28 x^{3}-25 x^{2}+14 x-1 \\
-x^{6}+2 x^{5}+4 x^{4}+36 x^{3}+25 x^{2}+42 x-59 \\
9 x^{6}+16 x^{5}+12 x^{4}-73 x^{3}-41 x^{2}+14 x+77 \\
-13 x^{5}+39 x^{4}+31 x^{3}-115 x^{2}-50 x-125 \\
-13 x^{5}+58 x^{4}-83 x^{3}-90 x^{2}+100 x+125 \\
5 x^{5}+30 x^{4}-61 x^{3}-122 x^{2}+112 x+161 \\
28 x^{6}+52 x^{5}-149 x^{4}-174 x^{3}+235 x^{2}-60 x-100 \\
8 x^{6}+192 x^{5}+237 x^{4}+238 x^{3}-15 x^{2}-60 x-76 \\
-56 x^{6}+132 x^{5}+102 x^{4}+195 x^{3}-240 x^{2}-204 x-178 \\
4 x^{6}-52 x^{5}+133 x^{4}+34 x^{3}-171 x^{2}-308 x-292 \\
-2 x^{6}-54 x^{5}+135 x^{4}+120 x^{3}-135 x^{2}-324 x-108 \\
-15 x^{6}+36 x^{5}+5 x^{4}+52 x^{3}+50 x^{2}+156 x+375 \\
4 x^{6}-12 x^{5}-219 x^{4}-460 x^{3}+15 x^{2}-246 x-230
\end{gathered}
$$

Table 6. Some points of small height on the surface of Theorem 21 and the corresponding genus- 2 curves. 


\section{Discriminant 24}

12.1. Parametrization. We start with an elliptic $\mathrm{K} 3$ surface with fibers of type $E_{6}$, $E_{7}$ and $A_{3}$ at $t=\infty, 0$ and 1 respectively.

A Weierstrass equation for such a family is given by $y^{2}=x^{3}+t^{2} x^{2}+a(t-1) t^{3}\left(2+\left(d^{2}-a+1\right)(t-1)\right) x+a^{2}(t-1)^{2} t^{5}\left(1+d^{2}(t-1)\right)$.

We identify a fiber of type $E_{8}$, and this leads us by a 3-neighbor step to an $E_{8} E_{7}$ fibration.

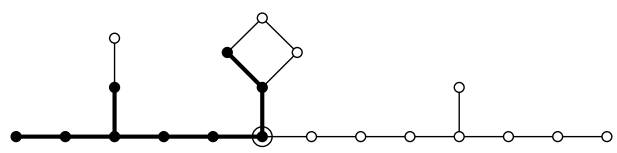

From the new elliptic fibration we read out the Igusa-Clebsch invariants as usual, and then obtain the branch locus of $Y_{-}(24)$ as a double cover of $\mathbb{P}_{a, d}^{2}$, which is a union of two curves: one corresponding to an extra $\mathrm{I}_{2}$ fiber, and one corresponding to the locus where the $E_{7}$ fiber promotes to an $E_{8}$ fiber.

Theorem 22. A birational model over $\mathbb{Q}$ for the Hilbert modular surface $Y_{-}(24)$ as a double cover of $\mathbb{P}_{a, d}^{2}$ is given by the equation

$$
z^{2}=\left(d^{2}-a-1\right)\left(16 a d^{4}-8 a^{2} d^{2}-20 a d^{2}+d^{2}+a^{3}-3 a^{2}+3 a-1\right) .
$$

It is a singular $\mathrm{K} 3$ surface.

12.2. Analysis. Note the extra involution $(a, d) \mapsto(a,-d)$.

The branch locus has two components. The first is the zero locus of $d^{2}-a-1$, and is obviously a rational curve (i.e., of genus 0 ) in the moduli space. It parametrizes the K3 surfaces in the family for which the $E_{7}$ fiber gets promoted to an $E_{8}$ fiber. The other component parametrizes elliptic $\mathrm{K} 3$ surfaces for which there is an extra $\mathrm{I}_{2}$ fiber. It is also a genus- 0 curve, though this fact is less obvious. A parametrization is given by

$$
(a, d)=\left(\frac{1-g^{2}}{2 g^{2}-1}, \frac{g^{3}}{2 g^{2}-1}\right) .
$$

The equation of the Hilbert modular surface describes it as a family of curves of genus 1 fibered over $\mathbb{P}_{d}^{1}$. In fact, we readily check that $(a, z)=\left(0, d^{2}-1\right)$ gives a section. So in fact, we have an elliptic surface, and by using the formula for the Jacobian, we can write it in Weierstrass form as

$$
\begin{aligned}
y^{2}= & x^{3}-x d^{2}\left(144 d^{6}-324 d^{4}+235 d^{2}-54\right) / 48 \\
& -d^{2}\left(3456 d^{10}-22032 d^{8}+50625 d^{6}-54866 d^{4}+28647 d^{2}-5832\right) / 1728 .
\end{aligned}
$$


This is an elliptic K3 surface $E$, and in fact, it is the base change (by $d \mapsto d^{2}$ ) of a rational elliptic surface with reducible fibers of types $\mathrm{I}_{2}, \mathrm{I}_{3}$ and $\mathrm{I}_{4}$ (and therefore with Mordell-Weil rank 2). We can use this to readily compute one section

$$
\left(x_{0}, y_{0}\right)=\left(11 d^{4}-239 d^{2} / 12+9,\left(d^{2}-1\right)\left(9 d^{2}-8\right)\left(32 d^{2}-27\right) / 8\right)
$$

of height $\frac{1}{6}$. Translating $x$ by $x_{0}$ and scaling to get rid of denominators, we get the following nicer form for $E$ :

$$
\begin{aligned}
& y^{2}=x^{3}+\left(132 d^{4}-239 d^{2}+108\right) x^{2} \\
& +2\left(d^{2}-1\right)\left(9 d^{2}-8\right)\left(32 d^{2}-27\right)\left(10 d^{2}-9\right) x+\left(\left(d^{2}-1\right)\left(9 d^{2}-8\right)\left(32 d^{2}-27\right)\right)^{2} .
\end{aligned}
$$

This has reducible fibers of type $\mathrm{I}_{2}$ at $d=\infty$ and $d^{2}=\frac{8}{9}$, type $\mathrm{I}_{3}$ at $d^{2}=\frac{27}{32}$, type IV at $d=0$, and type $\mathrm{I}_{4}$ at $d= \pm 1$. This gives a root system of type $A_{1}^{3} \oplus A_{2}^{3} \oplus A_{3}^{2}$, which has rank 15 and discriminant 1152.

In addition to the section $P_{1}=\left(0,\left(d^{2}-1\right)\left(9 d^{2}-8\right)\left(32 d^{2}-27\right)\right)$, we also find the sections

$$
\begin{aligned}
& P_{2}=\left(-5\left(d^{2}-1\right)\left(9 d^{2}-8\right), \sqrt{-1}\left(d^{2}-1\right)^{2}\left(9 d^{2}-8\right)\right), \\
& P_{3}=\left(-\left(32 d^{2}-27\right)\left(9 d^{2}-8\right) / 6, d\left(32 d^{2}-27\right)\left(9 d^{2}-8\right) / \sqrt{216}\right) .
\end{aligned}
$$

This shows that the elliptic K3 surface $Y_{-}$(24) has geometric Picard number 20, i.e., is a singular K3 surface. The discriminant of the span of the algebraic divisors exhibited is 96 . We showed that this is the entire Néron-Severi group by checking that our subgroup of the Mordell-Weil group is 2-saturated.

As mentioned, the quotient by the involution $d \mapsto-d$ is a rational elliptic surface

$$
\begin{aligned}
y^{2}=x^{3}+ & \left(132 t^{2}-239 t+108\right) x^{2} \\
+ & 2(t-1)(9 t-8)(32 t-27)(10 t-9) x+((t-1)(9 t-8)(32 t-27))^{2} .
\end{aligned}
$$

This surface has an $\mathrm{I}_{2}$ fiber at $t=\frac{8}{9}$, an $\mathrm{I}_{3}$ fiber at $t=\frac{27}{32}$ and an $\mathrm{I}_{4}$ fiber at $t=1$.

The Mordell-Weil group is generated by the sections

$$
\begin{aligned}
& P_{1}=(0,(t-1)(9 t-8)(32 t-27)), \\
& P_{2}=\left(-5(t-1)(9 t-8), \sqrt{-1}(t-1)^{2}(9 t-8)\right),
\end{aligned}
$$

with height matrix

$$
\left(\begin{array}{cc}
\frac{1}{12} & 0 \\
0 & \frac{1}{2}
\end{array}\right)
$$

12.3. Examples. Table 7 lists some points of small height and their genus- 2 curves.

For instance, for the genus- 2 curves corresponding to $\left(1, \frac{3}{2}\right)$ and $\left(1,-\frac{3}{2}\right)$, the point counts modulo $p$ match the twist by $\mathbb{Q}(\sqrt{2})$ of a modular form of level 2592 . 


\begin{tabular}{l|r} 
Rational point $(a, d)$ & Sextic polynomial $f_{6}(x)$ defining the genus-2 curve $y^{2}=f_{6}(x)$ \\
\hline$\left(\frac{77}{36},-\frac{1}{6}\right)$ & $-7 x^{6}-18 x^{4}-10 x^{3}+3 x^{2}+10 x+22$ \\
$\left(\frac{21}{16}, \frac{1}{8}\right)$ & $-12 x^{5}+8 x^{4}+24 x^{3}-16 x^{2}-21 x+14$ \\
$\left(\frac{9}{4},-\frac{1}{2}\right)$ & $3 x^{6}-12 x^{5}+20 x^{4}-79 x^{3}+11 x^{2}-84 x+60$ \\
$\left(-\frac{7}{81},-\frac{17}{18}\right)$ & $27 x^{6}-56 x^{5}-21 x^{4}+52 x^{3}+57 x^{2}-84 x+49$ \\
$\left(\frac{77}{36}, \frac{1}{6}\right)$ & $-32 x^{6}-80 x^{5}+94 x^{4}+115 x^{3}-91 x^{2}-55 x+33$ \\
$\left(\frac{9}{4},-\frac{5}{4}\right)$ & $-24 x^{6}+48 x^{5}+100 x^{4}+120 x^{3}-50 x^{2}-72 x-41$ \\
$\left(-\frac{3}{4},-\frac{1}{4}\right)$ & $25 x^{6}+70 x^{4}+24 x^{3}+124 x^{2}+48 x+72$ \\
$\left(\frac{9}{4}, \frac{1}{2}\right)$ & $-72 x^{6}+84 x^{5}+127 x^{4}-123 x^{3}-83 x^{2}+51 x+25$ \\
$\left(1, \frac{3}{2}\right)$ & $9 x^{6}+9 x^{4}-60 x^{3}-45 x^{2}+132 x-53$ \\
$\left(\frac{33}{50},-\frac{1}{5}\right)$ & $50 x^{5}-50 x^{4}+35 x^{3}-35 x^{2}-31 x+139$ \\
$\left(-\frac{3}{4}, \frac{1}{4}\right)$ & $33 x^{6}-36 x^{5}+110 x^{4}-120 x^{3}+140 x^{2}-96 x+72$ \\
$\left(\frac{21}{16},-\frac{1}{8}\right)$ & $7 x^{5}+3 x^{4}+78 x^{3}-2 x^{2}+63 x+147$ \\
$\left(21,-\frac{5}{2}\right)$ & $-8 x^{6}-24 x^{5}+80 x^{4}-100 x^{3}+170 x^{2}-84 x+63$ \\
$\left(-\frac{4}{9}, \frac{2}{3}\right)$ & $-3 x^{6}+14 x^{5}-63 x^{4}+96 x^{3}-171 x^{2}-48 x-188$ \\
$\left(-\frac{7}{81}, \frac{17}{18}\right)$ & $-7 x^{6}+56 x^{5}-95 x^{4}-20 x^{3}-205 x^{2}-44 x-93$ \\
$\left(21, \frac{5}{2}\right)$ & $-24 x^{6}-24 x^{5}-280 x^{4}-260 x^{3}+170 x^{2}-24 x+1$ \\
\hline
\end{tabular}

Table 7. Some points of small height on the surface of Theorem 22 and the corresponding genus- 2 curves.

We describe a few curves on the Hilbert modular surface, which can be used to produce rational points. Setting $a=-\frac{1}{9}$ gives a rational curve of genus 0 , with infinitely many points. Sections of the fibration will also lead to curves birational to $\mathbb{P}^{1}$ over $\mathbb{Q}$. For instance, $P_{1}$ and $2 P_{1}$ describe the rational curves given by $a=4\left(d^{2}-1\right) / 5$ and $a=\left(4 d^{2}-5\right) / 13$, respectively. The Brauer obstruction does not vanish identically on any of these.

\section{Discriminant 28}

13.1. Parametrization. We start with an elliptic $\mathrm{K} 3$ surface with fibers of type $E_{6}, D_{5}$ and $A_{4}$ at $t=\infty, 0$ and 1 respectively, and a section of height $\frac{28}{60}=\frac{7}{15}=$ $4-\frac{6}{5}-1-\frac{4}{3}$.

A Weierstrass equation for this family is given by

$$
y^{2}=x^{3}+a t x^{2}+b t^{2}(t-1)^{2} x+c t^{3}(t-1)^{4},
$$


where

$$
\begin{aligned}
& a=2\left(f^{2}-g^{2}\right)(t-1)+t, \\
& b=\left(f^{2}-g^{2}\right)^{2}(1-t)-2\left(f^{2}-g^{2}\right)(f+1) t, \\
& c=(f+1)^{2}\left(f^{2}-g^{2}\right) t .
\end{aligned}
$$

We identify the class of a $D_{8}$ fiber and carry out a 2-neighbor step to convert to an elliptic fibration with $D_{8}$ and $E_{7}$ fibers, and a section of height $\frac{7}{2}=4+2 \cdot 1-1-\frac{3}{2}$.

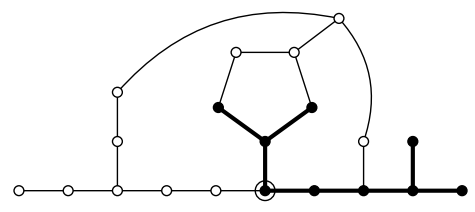

Then we identify the class of an $E_{8}$ fiber, and carry out a 2-neighbor step to get the desired $E_{8} E_{7}$ fibration.

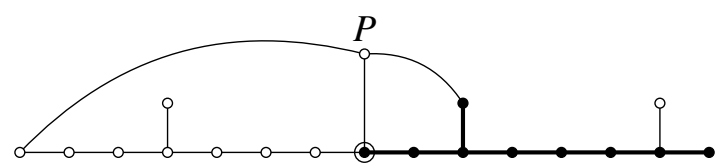

The new elliptic fibration has a section, since $P \cdot F^{\prime}=5$, while the remaining component of the $D_{8}$ fiber has intersection number 2 with $F^{\prime}$. We now read out the Igusa-Clebsch invariants.

Theorem 23. A birational model over $\mathbb{Q}$ for the Hilbert modular surface $Y_{-}(28)$ as a double cover of $\mathbb{P}_{g, h}^{2}$ is given by the equation

$$
\begin{aligned}
z^{2}=- & (g-f-2)(g+f+2) \\
& \times\left(8 g^{4}+92 f^{2} g^{2}+180 f g^{2}+71 g^{2}-100 f^{4}-180 f^{3}-71 f^{2}+4 f+4\right) .
\end{aligned}
$$

It is a singular $\mathrm{K} 3$ surface.

13.2. Analysis. It has a second involution $(f, g) \mapsto(f,-g)$. The branch locus consists of three components. The factors $g \pm(f+2)$ correspond to the locus where the Picard number of the K3 surface jumps to 19, due to the presence of an $\mathrm{I}_{2}$ fiber, but the discriminant decreases to 14 , because the nontrivial section of height $\frac{7}{15}$ becomes divisible by 2 . The more complicated factor corresponds to just the presence of an extra $I_{2}$ factor, which makes the discriminant 56 . This component of the branch locus is also a genus 0 curve; a parametrization is given by

$(f, g)=\left(-\frac{2 m^{4}+17 m^{3}+57 m^{2}+85 m+47}{2(m+1)(m+2)\left(m^{2}+6 m+11\right)},-\frac{\left(m^{2}+6 m+7\right)\left(2 m^{2}+7 m+7\right)}{2(m+1)(m+2)\left(m^{2}+6 m+11\right)}\right)$. 
Rational point $(f, g) \quad$ Sextic polynomial $f_{6}(x)$ defining the genus-2 curve $y^{2}=f_{6}(x)$

\begin{tabular}{l|c}
$\left(-\frac{31}{16},-\frac{9}{16}\right)$ & $9 x^{6}-15 x^{5}+39 x^{4}-25 x^{3}+36 x^{2}+11$ \\
$\left(-\frac{56}{65},-\frac{61}{65}\right)$ & $-37 x^{6}+42 x^{5}+17 x^{4}-33 x^{3}-7 x^{2}-3 x-3$ \\
$\left(-\frac{31}{16}, \frac{9}{16}\right)$ & $15 x^{5}-39 x^{4}-11 x^{3}+45 x^{2}+27 x$ \\
$\left(-\frac{35}{24},-\frac{31}{24}\right)$ & $-27 x^{6}+54 x^{5}-36 x^{4}+30 x^{3}-24 x^{2}-6 x-2$ \\
$\left(-\frac{12}{11},-\frac{1}{11}\right)$ & $x^{6}+14 x^{5}+61 x^{4}+73 x^{3}-49 x^{2}+3 x+1$ \\
$\left(-\frac{14}{9},-\frac{5}{9}\right)$ & $37 x^{6}+69 x^{5}+75 x^{4}+79 x^{3}+69 x^{2}+12 x+20$ \\
$\left(-\frac{2}{5},-\frac{7}{5}\right)$ & $-4 x^{6}+12 x^{5}-x^{4}+87 x^{3}+68 x^{2}+48 x+3$ \\
$\left(-\frac{13}{8},-\frac{7}{8}\right)$ & $3 x^{6}-6 x^{5}+5 x^{4}-10 x^{3}+41 x^{2}+30 x+87$ \\
$\left(-\frac{42}{41}, 1\right)$ & $-92 x^{6}-76 x^{5}+21 x^{4}+9 x^{3}+9 x^{2}-x-1$ \\
$\left(-\frac{13}{8}, \frac{7}{8}\right)$ & $18 x^{6}+78 x^{5}+44 x^{4}-94 x^{3}-76 x^{2}+30 x+25$ \\
$\left(\frac{5}{16},-\frac{19}{16}\right)$ & $-48 x^{6}+45 x^{5}+11 x^{4}+87 x^{3}-97 x^{2}-63 x+56$ \\
$\left(-\frac{30}{41},-\frac{36}{41}\right)$ & $-27 x^{6}+57 x^{5}-100 x^{4}-68 x^{3}-76 x^{2}+36 x$ \\
$\left(-\frac{11}{3}, \frac{7}{3}\right)$ & $16 x^{6}-24 x^{5}-111 x^{4}+9 x^{3}+102 x^{2}-27 x-33$ \\
$\left(\frac{26}{31},-\frac{64}{31}\right)$ & $-9 x^{6}-6 x^{5}+32 x^{4}+32 x^{3}-112 x^{2}-6 x+99$ \\
$\left(\frac{13}{80},-\frac{15}{16}\right)$ & $-9 x^{6}-15 x^{5}+85 x^{3}-135 x+54$ \\
$\left(\frac{26}{31}, \frac{64}{31}\right)$ & $-25 x^{6}+30 x^{5}-64 x^{4}+72 x^{3}-136 x^{2}+102 x-69$ \\
\hline
\end{tabular}

Table 8. Some points of small height on the surface of Theorem 23 and the corresponding genus- 2 curves.

Since the double cover is branched along a sextic, the Hilbert modular surface is itself a K3 surface. Setting $f=h-2$ and then using the invertible substitution $h=t(1+1 / x), g=t(1-1 / x)$ (and absorbing square factors) converts it to an elliptic fibration over $\mathbb{P}_{t}^{1}$, which we can write in Weierstrass form as

$$
\begin{aligned}
y^{2}= & x^{3}-t\left(108 t^{3}-176 t^{2}+63 t+4\right) x^{2} \\
& +32(t-1)^{2} t^{3}\left(135 t^{2}-36 t-106\right) x-64(t-1)^{4} t^{4}\left(6075 t^{2}-6075 t-196\right) .
\end{aligned}
$$

This has fibers of type $\mathrm{I}_{2}$ at $t=\infty, \frac{4}{5}$ and $\frac{28}{27}, \mathrm{I}_{1}^{*}$ at $t=0, \mathrm{I}_{5}$ at $t=1$, and $\mathrm{I}_{3}$ at $t=(19 \pm 7 \sqrt{7}) / 36$, giving a contribution of $D_{5} \oplus A_{4} \oplus A_{1}^{3} \oplus A_{2}^{2}$ to the Néron-Severi lattice. Therefore the Picard number is at least 18 . We identify the sections

$$
\begin{aligned}
& P_{1}=\left(12 t^{2}(t-1)(36 t-37), 4 t^{2}(t-1)(27 t-28)\left(72 t^{2}-76 t+1\right)\right), \\
& P_{2}=\left(t\left(1080 t^{3}-2064 t^{2}+953 t+28\right) / 7,\right. \\
& \left.2 t^{2}(5 t-4)(27 t-28)\left(72 t^{2}-76 t+1\right) / 7^{3 / 2}\right),
\end{aligned}
$$

with height matrix

$$
\left(\begin{array}{cc}
\frac{7}{60} & 0 \\
0 & \frac{2}{3}
\end{array}\right) .
$$


Therefore the Picard number is 20. An easy lattice-theoretic argument (see the online supplement) shows that these sections must generate the Mordell-Weil group, and therefore the Néron-Severi lattice has discriminant 112.

The quotient by the involution $g \mapsto-g$ has equation

$$
\begin{array}{r}
y^{2}=x^{3}-\left(84 f^{2}+148 f+39\right) x^{2} / 4-\left(96 f^{4}+364 f^{3}+615 f^{2}+500 f+140\right) x \\
-(f+2)^{2}(5 f+2)^{2}\left(4 f^{2}+4 f-1\right) .
\end{array}
$$

This is a rational elliptic surface with an $\mathrm{I}_{4}$ fiber at $f=-1$, an $\mathrm{I}_{3}$ fiber at $f=-\frac{17}{18}$, and $\mathrm{I}_{2}$ fibers at $t=-\frac{26}{27}$ and $t=\infty$. The Mordell-Weil group is generated by the 2-torsion section $\left(-2(f+2)^{2}, 0\right)$ and the non-torsion section $\left(-2\left(f^{2}+3 f+3\right),(f+1)(18 f+17)\right)$ of height $\frac{1}{12}$.

13.3. Examples. Table 8 lists some points of small height and their genus- 2 curves.

Next, we describe some special curves on the surface, which may be used to produce rational points. First, $f=-\frac{17}{18}$ gives a rational curve, which can be parametrized as $g=-19\left(h^{2}-2\right) /\left(18\left(h^{2}+2\right)\right)$. The Brauer obstruction vanishes identically for this family, giving a family of genus- 2 curves with real multiplication by $\mathcal{O}_{28}$. Next, the specialization $f=-\frac{26}{27}$ gives another rational curve, which can be parametrized as

$$
g=-2 \frac{13 h^{2}+729 h-75816}{27\left(h^{2}+5832\right)} .
$$

The Brauer obstruction does not vanish identically for this family. Finally, the section $P_{1}$ on the Jacobian of $Y_{-}(28)$ can be described as

$$
(f, g)=\left(\frac{4 t^{2}-8 t+1}{4 t-1}, \frac{4 t^{2}-2 t+1}{4 t-1}\right) .
$$

The Brauer obstruction vanishes identically on this family as well.

From a plot of the rational points, we observe many rational points on the lines $g= \pm(5 f+2) / 3$. However, the Brauer obstruction does not vanish identically along these lines.

\section{Discriminant 29}

14.1. Parametrization. We start with an elliptic $\mathrm{K} 3$ surface with fibers of type $E_{7}$ and $A_{8}$, and a section $P$ of height $\frac{29}{18}=4-\frac{3}{2}-\frac{8}{9}$. A Weierstrass equation for this family is given by

$$
\begin{aligned}
& y^{2}=x^{3}+\left(-(4 f-1) t^{2}+(g-2) t+1\right) x^{2} \\
&-2 g t^{3}\left(2 f^{2} t^{2}+(-g+2 f+1) t-1\right) x+g^{2} t^{6}((g-4 f) t+1) .
\end{aligned}
$$


We identify a fiber $F$ of type $E_{8}$, and go to the associated elliptic fibration by a 3-neighbor step. Note that $P \cdot F=4$, while the remaining component of the $A_{8}$ fiber intersects $F$ with multiplicity 3 . Therefore the new fibration has a section.

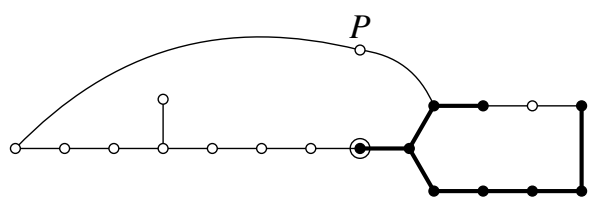

The new elliptic fibration has $E_{8}$ and $E_{7}$ fibers, and we may read out the IgusaClebsch invariants. The branch locus for the double cover of $\mathbb{P}_{f, g}^{2}$ corresponds to elliptic $\mathrm{K} 3$ surfaces having an extra $\mathrm{I}_{2}$ fiber.

Theorem 24. A birational model over $\mathbb{Q}$ for the Hilbert modular surface $Y_{-}(29)$ as a double cover of $\mathbb{P}^{2}$ is given by the equation

$$
\begin{aligned}
z^{2}=-g^{4}+(6 f+11) g^{3}-( & \left.27 f^{4}-18 f^{3}+5 f^{2}+102 f-1\right) g^{2} \\
& +8 f\left(36 f^{3}-25 f^{2}+35 f-1\right) g-16 f^{2}(4 f-1)^{3} .
\end{aligned}
$$

It is a $\mathrm{K} 3$ surface of Picard number 19.

14.2. Analysis. The branch locus is a rational curve, and a parametrization is given by

$$
(f, g)=\left(\frac{(m+2)^{2}\left(m^{2}+2 m-4\right)}{m^{2}\left(m^{2}+6 m+12\right)}, \frac{4(m+2)^{2}(m+4)^{3}}{m\left(m^{2}+6 m+12\right)^{2}}\right) .
$$

The substitution $g=h f$ makes the right-hand side of the equation a quartic in $f$, after absorbing a factor of $f^{2}$ into $z^{2}$ on the left. Also, $f=0$ makes the right-hand side a square (namely $(h-4)^{2}$ ), giving us a point on the genus- 1 curve over $\mathbb{P}_{h}^{1}$. We may then replace the genus- 1 curve by its Jacobian, which has the following equation after the change of parameter $h=4-t$, and some Weierstrass transformations:

$$
\begin{aligned}
y^{2}= & x^{3}-\left(t^{4}-10 t^{3}-t^{2}-t-16\right) x^{2} \\
& -t\left(20 t^{5}-140 t^{4}-3740 t^{3}+14234 t^{2}+3349 t-1120\right) x \\
& -t^{2}\left(3475 t^{6}-46300 t^{5}+180355 t^{4}-10362 t^{3}-849193 t^{2}+276269 t-19600\right) .
\end{aligned}
$$

This is an elliptic K3 surface, with bad fibers of type $\mathrm{I}_{4}$ at $t=\infty, \mathrm{I}_{3}$ at $t=0$ and at the roots of the polynomial $15 t^{3}+12 t^{2}+160 t-64$ (which gives the cubic field of discriminant -87), and $\mathrm{I}_{2}$ at the roots of $2 t^{3}-38 t^{2}+255 t-28$ (which gives the cubic field of discriminant -116). The trivial lattice therefore has rank 16, leaving room for Mordell-Weil rank at most 4. We find the linearly independent sections $P_{1}=\left(t\left(125 t^{3}-700 t^{2}+800 t-76\right) / 4\right.$, 


$$
\begin{aligned}
& t\left.\left(1375 t^{5}-11450 t^{4}+29240 t^{3}-23616 t^{2}+7296 t-512\right) / 8\right), \\
& P_{2}=\left(\left(30 t^{4}-306 t^{3}+636 t^{2}+1833 t-448\right) / 29,\right.\left.\left(2 t^{3}-38 t^{2}+255 t-28\right)\left(15 t^{3}+12 t^{2}+160 t-64\right) /(29 b)\right), \\
& P_{3}=\left(2(3 a-5) t^{2}-3(8 a-23) t,\right. \\
&\left.a t\left(-99 t^{3}+18(a+37) t^{2}-144(3 a+7) t+416 a-288\right) / 3\right) .
\end{aligned}
$$

Here $a=\sqrt{-3}$ and $b=\sqrt{29}$. Therefore, the Picard number of the Hilbert modular surface is at least 19 . We showed by counting points modulo 11 and 13 that the Picard number must be exactly 19. This agrees with the calculations in [Oda 1982, p. 109]. The height matrix of the sections above is

$$
\frac{1}{6}\left(\begin{array}{rrr}
20 & 0 & -10 \\
0 & 3 & 0 \\
-10 & 0 & 14
\end{array}\right) \text {. }
$$

Therefore, the sublattice of the Néron-Severi lattice spanned by the sections above together with the trivial lattice has discriminant $2^{4} \cdot 3^{4} \cdot 5=6480$. By checking that it is 2- and 3-saturated, we showed that it is the entire Néron-Severi lattice, and therefore the sections $P_{1}, P_{2}, P_{3}$ generate the Mordell-Weil group.

14.3. Examples. Table 9 lists some points of small height and their genus- 2 curves.

The section $P_{1}$ corresponds to the rational curve given by $g=\frac{4}{5} u f$, with

$$
f=\frac{22 u^{5}-321 u^{4}+1651 u^{3}-3377 u^{2}+1980 u+50}{25 u^{6}-400 u^{5}+2375 u^{4}-6050 u^{3}+4813 u^{2}+2325 u+225} .
$$

The Brauer obstruction vanishes identically, yielding a 1-parameter family of genus2 curves with real multiplication by $\mathcal{O}_{29}$.

\section{Discriminant 33}

15.1. Parametrization. We start with an elliptic $\mathrm{K} 3$ surface with fibers of type $A_{10}$ and $E_{6}$ at $t=\infty$ and $t=0$ respectively. A Weierstrass equation for such a family is given by

$$
y^{2}=x^{3}+(c+2 d+1) t^{2} x^{2}+2(c+d) t^{4} x+c t^{4},
$$

with

$$
c=\left(s^{2}-r^{2}\right)^{2} t^{2}-\left(s^{2}-r^{2}\right)\left(s^{2}-r^{2}+2 r\right) t+s^{2}, \quad d=\left(s^{2}-r^{2}\right)(1-t)+r .
$$

We identify the class of an $E_{7}$ fiber below, and perform a 2-neighbor step.

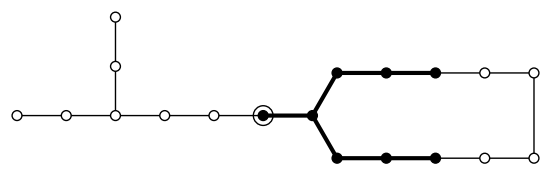


The new elliptic fibration has fibers of type $E_{6}, E_{7}$ and $A_{2}$, as well as a section $P$ of height $\frac{33}{18}=\frac{11}{6}=4-0-\frac{3}{2}-\frac{2}{3}$. We then identify the class of an $E_{8}$ fiber and carry out a 3-neighbor step to an $E_{8} E_{7}$ elliptic fibration.

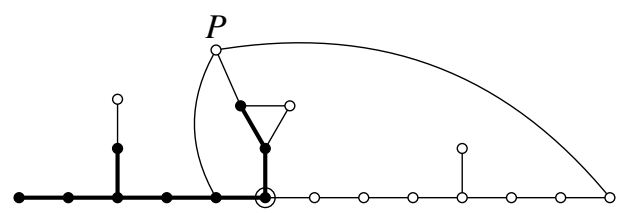

The new elliptic fibration has a section, since $P \cdot F^{\prime}=5$, while the intersection number of the remaining component of the $E_{6}$ fiber with $F^{\prime}$ is 3 , and these are coprime.

From the Weierstrass equation of the $E_{8} E_{7}$ fibration, we determine the IgusaClebsch invariants, and then the equation of the branch locus.

Theorem 25. A birational model over $\mathbb{Q}$ for the Hilbert modular surface $Y_{-}(33)$ as a double cover of $\mathbb{P}^{2}$ is given by the equation

$$
\begin{aligned}
z^{2}=9 s^{6}-\left(26 r^{2}-80 r+104\right) s^{4}+\left(25 r^{4}-152 r^{3}+400 r^{2}-408 r+432\right) s^{2} \\
-\left(8 r^{6}-72 r^{5}+280 r^{4}-472 r^{3}+336 r^{2}-64 r-16\right) .
\end{aligned}
$$

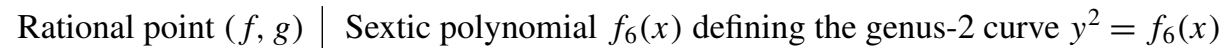

\begin{tabular}{l|c}
$\left(-37,-\frac{37}{2}\right)$ & $-98 x^{5}-56 x^{4}-131 x^{3}-114 x^{2}+10 x-68$ \\
$\left(\frac{20}{67}, \frac{16}{67}\right)$ & $264 x^{6}+760 x^{5}+183 x^{4}-630 x^{3}-53 x^{2}-20 x-4$ \\
$\left(\frac{6}{7}, \frac{48}{7}\right)$ & $12 x^{6}-12 x^{5}-409 x^{4}+1062 x^{3}+287 x^{2}-588 x-252$ \\
$(-40,40)$ & $-53 x^{6}+227 x^{5}+374 x^{4}+1191 x^{3}+669 x^{2}+680 x+900$ \\
$\left(-1,-\frac{29}{10}\right)$ & $1210 x^{5}-110 x^{4}+511 x^{3}-17 x^{2}+53 x+1$ \\
$\left(-4,-\frac{16}{5}\right)$ & $-200 x^{6}+1360 x^{5}-995 x^{4}+242 x^{3}-191 x^{2}-4 x-12$ \\
$\left(-\frac{1}{7},-\frac{37}{98}\right)$ & $-1588 x^{6}+986 x^{5}-122 x^{4}+221 x^{3}-68 x^{2}-2 x-8$ \\
$(-6,8)$ & $540 x^{6}+2052 x^{5}-1149 x^{4}+1724 x^{3}-39 x^{2}-894 x-506$ \\
$\left(\frac{5}{43}, \frac{19}{43}\right)$ & $-2 x^{6}+80 x^{5}-786 x^{4}+2265 x^{3}+74 x^{2}+80 x-2$ \\
$\left(\frac{8}{9}, \frac{16}{9}\right)$ & $-4 x^{6}+204 x^{5}-837 x^{4}-160 x^{3}+2451 x^{2}+1620 x-228$ \\
$\left(-\frac{6}{13},-\frac{16}{13}\right)$ & $236 x^{6}-796 x^{5}+2293 x^{4}-2178 x^{3}+1525 x^{2}+2492 x-764$ \\
$\left(-\frac{4}{9}, \frac{16}{9}\right)$ & $552 x^{6}-2232 x^{5}+3183 x^{4}+562 x^{3}-4713 x^{2}-1248 x-88$ \\
$\left(-3, \frac{36}{5}\right)$ & $1024 x^{6}-1920 x^{5}-2252 x^{4}+1065 x^{3}-2288 x^{2}-6195 x+66$ \\
$\left(\frac{18}{19}, \frac{48}{19}\right)$ & $-768 x^{6}-2560 x^{5}+1571 x^{4}+7838 x^{3}-2133 x^{2}-6912 x+2376$ \\
$\left(-\frac{4}{5},-\frac{32}{25}\right)$ & $-1412 x^{6}-1372 x^{5}+2149 x^{4}+8226 x^{3}+4889 x^{2}-896 x-4096$ \\
$\left(\frac{2}{9}, \frac{7}{9}\right)$ & $3189 x^{6}+4599 x^{5}-6897 x^{4}-9331 x^{3}+5424 x^{2}+5040 x-1968$ \\
\hline
\end{tabular}

Table 9. Some points of small height on the surface of Theorem 24 and the corresponding genus- 2 curves. 
It is a singular $\mathrm{K} 3$ surface.

15.2. Analysis. This is a double cover of the $(r, s)$-plane branched along a sextic, and is therefore a $\mathrm{K} 3$ surface. The extra involution is given by $(r, s) \mapsto(r,-s)$. The equation of the branch locus may be transformed as follows: setting $t=s^{2}$, we have

$$
\begin{array}{r}
-8 r^{6}+72 r^{5}+(25 t-280) r^{4}+(-152 t+472) r^{3}+\left(-26 t^{2}+400 t-336\right) r^{2} \\
+\left(80 t^{2}-408 t+64\right) r+\left(9 t^{3}-104 t^{2}+432 t+16\right)=0
\end{array}
$$

which, after resolution of singularities, becomes a genus- 0 curve, parametrized by

$$
r=\frac{m^{3}+4 m^{2}+4 m+4}{m^{2}(m+1)}, \quad t=\frac{8\left(m^{3}+4 m^{2}+4 m+2\right)}{m^{4}(m+1)^{2}} .
$$

Then the branch locus can be written as a double cover

$$
s^{2}=8\left(m^{3}+4 m^{2}+4 m+2\right) /\left(m^{4}(m+1)^{2}\right) .
$$

After removing square factors and performing a Weierstrass transformation, it is converted to the elliptic curve $y^{2}+y=x^{3}-x^{2}$. It is isomorphic to $X_{1}(11) \cong$ $X_{0}(33) /\left\langle w_{33}\right\rangle$, where $w_{33}$ is the Atkin-Lehner involution.

For the equation of the Hilbert modular surface, the transformation $s=r+t$ makes the right-hand side of the equation a quartic in $r$, with the coefficient of $r^{4}$ being a square. Converting to the Jacobian form, and applying a Weierstrass transformation as well as scaling $t$, we get an elliptic fibration

$$
\begin{aligned}
& y^{2}=x^{3}+\left(t^{4}+24 t^{3}+58 t^{2}+84 t+1\right) x^{2} \\
&+\left(280 t^{5}+5488 t^{4}+1376 t^{3}+2192 t^{2}+72 t\right) x \\
&+ 4608 t^{7}+95632 t^{6}+32576 t^{5}+26848 t^{4}+14656 t^{3}+1296 t^{2} .
\end{aligned}
$$

This has bad fibers of type $\mathrm{I}_{5}$ at $t=\infty$, type II at $t=1$, type $\mathrm{I}_{3}$ at $t=0, \frac{1}{2}$ and $(-17 \pm 3 \sqrt{33}) / 2$, and type $\mathrm{I}_{2}$ at $t=-\frac{21}{2} \pm \frac{11 \sqrt{33}}{6}$. These contribute $A_{1}^{3} \oplus A_{2}^{4} \oplus A_{4}$ to the Néron-Severi lattice.

By finding sections modulo a small prime and attempting to lift them to $\mathbb{Q}$ or $\mathbb{Q}(\sqrt{33})$, we find the following sections of small height:

$$
\begin{aligned}
& P_{1}=\left(4 t\left(3 t^{2}+60 t-13\right), 4 t\left(t^{2}+17 t-2\right)\left(3 t^{2}+63 t-2\right)\right), \\
& P_{2}=\left(-4 t(3 t+11), 4 t(t-1)\left(3 t^{2}+63 t-2\right)\right), \\
& P_{3}=\left((4 \sqrt{33}+12) t^{2}-(2 \sqrt{33}+34) t,\right. \\
& \quad(\sqrt{33}+3) t(2 t-1)(2 t+3 \sqrt{33}+17)(6 t+63-11 \sqrt{33}) / 6) .
\end{aligned}
$$




\begin{tabular}{l|c} 
Rational point $(r, s)$ & Sextic polynomial $f_{6}(x)$ defining the genus-2 curve $y^{2}=f_{6}(x)$ \\
\hline$\left(\frac{11}{3}, \frac{8}{3}\right)$ & $-9 x^{6}-6 x^{5}-7 x^{4}+7 x^{3}+2 x^{2}+3 x-2$ \\
$(1,3)$ & $-4 x^{6}+15 x^{4}-x^{3}-9 x^{2}+12 x+5$ \\
$\left(-\frac{13}{5}, \frac{27}{5}\right)$ & $-14 x^{5}+20 x^{4}+2 x^{3}-15 x^{2}-4 x$ \\
$\left(\frac{28}{3}, \frac{23}{3}\right)$ & $-5 x^{6}+6 x^{5}-5 x^{4}+27 x^{3}-11 x^{2}+12 x-24$ \\
$\left(\frac{73}{19},-\frac{41}{19}\right)$ & $-7 x^{6}+15 x^{5}+x^{4}-31 x^{3}-2 x^{2}+12 x+12$ \\
$(1,-3)$ & $-x^{6}-3 x^{5}+9 x^{4}+34 x^{3}-30 x^{2}-9 x+8$ \\
$\left(\frac{41}{51}, \frac{7}{51}\right)$ & $-9 x^{6}-9 x^{5}-35 x^{4}+11 x^{3}-8 x^{2}+12 x$ \\
$\left(\frac{16}{15}, \frac{1}{15}\right)$ & $2 x^{6}-3 x^{5}+7 x^{4}+13 x^{3}-20 x^{2}+36 x-15$ \\
$\left(-\frac{13}{5},-\frac{27}{5}\right)$ & $-5 x^{5}-3 x^{4}+13 x^{3}-17 x^{2}-40 x$ \\
$\left(\frac{46}{3}, \frac{41}{3}\right)$ & $-8 x^{6}+36 x^{5}-23 x^{4}-21 x^{3}-47 x^{2}-18 x-9$ \\
$\left(-\frac{17}{10}, \frac{27}{10}\right)$ & $-20 x^{5}+28 x^{4}-37 x^{3}+60 x^{2}+44 x$ \\
$\left(\frac{13}{3},-\frac{11}{3}\right)$ & $7 x^{6}-48 x^{5}+68 x^{4}-2 x^{3}-25 x^{2}+24 x-36$ \\
$\left(-\frac{38}{11}, \frac{61}{11}\right)$ & $-9 x^{6}-6 x^{5}+69 x^{4}-19 x^{3}-39 x^{2}+12 x-8$ \\
$\left(-\frac{29}{3},-11\right)$ & $-31 x^{5}+71 x^{4}-32 x^{3}+23 x^{2}-40 x-8$ \\
$\left(\frac{1}{33}, \frac{56}{33}\right)$ & $3 x^{6}+24 x^{5}+8 x^{4}+41 x^{3}+32 x^{2}-72 x-36$ \\
$\left(\frac{13}{3}, \frac{11}{3}\right)$ & $-12 x^{6}+24 x^{5}-31 x^{4}+81 x^{3}-67 x^{2}+39 x-70$ \\
\hline &
\end{tabular}

Table 10. Some points of small height on the surface of Theorem 25 and the corresponding genus- 2 curves.

These are linearly independent in the Mordell-Weil group, and the matrix of NéronTate heights is

$$
\frac{1}{30}\left(\begin{array}{rrr}
6 & -2 & 3 \\
-2 & 19 & -1 \\
3 & -1 & 9
\end{array}\right)
$$

It has determinant $\frac{11}{360}$, and so the sublattice of the Néron-Severi group generated by these sections and the trivial lattice has rank 20 and discriminant $-\frac{11}{360} \cdot 2^{3} \cdot 3^{4} \cdot 5=$ -99. We show that this is the full Picard group by checking that our subgroup of the Mordell-Weil group is 3-saturated. We deduce that $Y_{-}(33)$ is a singular K3 surface with Picard lattice of discriminant $\mathbf{- 9 9}$.

The quotient of the $Y_{-}$(33) by the involution $s \mapsto-s$ has Weierstrass equation

$$
\begin{aligned}
y^{2}=x^{3}-2\left(13 r^{2}-40 r+52\right) x^{2}+9\left(25 r^{4}-\right. & \left.152 r^{3}+400 r^{2}-408 r+432\right) x \\
& -648(r-1)^{3}\left(r^{3}-6 r^{2}+14 r+2\right) .
\end{aligned}
$$

It is a rational elliptic surface, with reducible fibers of types $\mathrm{I}_{5}, \mathrm{I}_{3}$ and $\mathrm{I}_{2}$ at $r=\infty, 19$ and 23 respectively. The Mordell-Weil group is generated by the section $\left(3\left(3 t^{2}-16 t+112\right), 12(r-23)(r-19)\right)$ of height $\frac{1}{30}$. 
15.3. Examples. Table 10 on the previous page lists some points of small height and their genus- 2 curves.

We may attempt to match up these examples with eigenforms in the tables of modular forms. For instance, for the points $(1,3)$ and $(1,-3)$, the corresponding genus-2 curves (with isogenous Jacobians)

$$
\begin{aligned}
& y^{2}=-\left(x^{3}-3 x-1\right)\left(4 x^{3}-3 x+5\right), \\
& y^{2}=-\left(x^{3}-3 x^{2}+1\right)\left(x^{3}+6 x^{2}+9 x-8\right)
\end{aligned}
$$

have the property that their traces match those of a newform of weight $1296=2^{4} \cdot 3^{4}$ in the modular forms database.

We also see some simple rational curves on the Hilbert modular surface: the specialization $r=19$ gives a rational curve parametrized by $s=-\left(16 m^{2}+41\right) /(3 m)$, while $r=23$ is also a rational curve, parametrized by $s=-\left(16 m^{2}+7\right) / m$. The Brauer obstructions do not identically vanish for points on these curves.

We have slightly better luck with sections of the elliptic fibration: for instance, the sections $P_{1}, 2 P_{1}, 3 P_{1}$ give rise to rational curves with respective parametrizations

$$
\begin{gathered}
\left(-\frac{3 t^{2}-112}{2(3 t+8)}, \frac{3 t^{2}+16 t+112}{2(3 t+8)}\right), \quad\left(-\frac{t^{2}-12}{2(t+2)}, \frac{t^{2}+4 t+12}{2(t+2)}\right), \\
\left(-\frac{3 t^{2}-20}{6(t+2)}, \frac{3 t^{2}+12 t+20}{6(t+2)}\right) .
\end{gathered}
$$

The Brauer obstruction vanishes identically on each of these.

\section{Discriminant 37}

16.1. Parametrization. We start with an elliptic $\mathrm{K} 3$ surface with fibers of type $E_{6}$, $D_{5}$ and $A_{4}$ at $t=\infty, 0$ and 1 respectively, and a section of height $\frac{37}{60}=4-\frac{4}{3}-\frac{5}{4}-\frac{4}{5}$.

A Weierstrass equation for this family is

$$
y^{2}=x^{3}+a t x^{2}+b t^{2}(t-1)^{2} x+c t^{3}(t-1)^{4},
$$

with

$$
\begin{aligned}
& a=(2 g-f+1)(t-1)+g^{2} t / 4, \\
& b=(f-g-1)((f+g-1)(t-1)+(f-2) g t / 2), \\
& c=(g-f+1)^{2}\left(f^{2}(t-1)+(f-2)^{2}\right) / 4 .
\end{aligned}
$$

We identify the class of an $D_{8}$ fiber and carry out a 2-neighbor step to convert to an elliptic fibration with $D_{8}$ and $E_{6}$ fibers. 


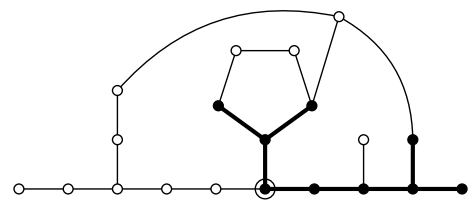

This new elliptic fibration has Mordell-Weil rank 2. In fact, it is quite easy to exhibit one non-torsion section $P$ (we do so in the online supplement) of height $\frac{2}{3}=4-\frac{4}{3}-2$. Then we find an $E_{8}$ fiber as shown below, and proceed to it by a 2-neighbor step. The section $P$ combined with most of the components of the $E_{6}$ fiber gives a disjoint $E_{7}$ configuration, and therefore the new elliptic fibration has reducible fibers of type $E_{8}$ and $E_{7}$. The fact that $(-P)$ intersects the multiplicity-1 component of the $E_{8}$ fiber shown below implies that the new fibration has a section.

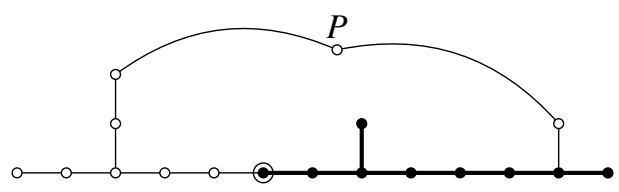

We then read out the Igusa-Clebsch invariants and write down the equation of the Hilbert modular surface.

Theorem 26. A birational model over $\mathbb{Q}$ for the Hilbert modular surface $Y_{-}(37)$ as a double cover of $\mathbb{P}^{2}$ is given by the equation

$$
\begin{array}{rl}
z^{2}=f^{2} g^{4}+2 & f(14 f-1) g^{3}-\left(126 f^{3}-142 f^{2}+44 f-1\right) g^{2} \\
+ & (f-1)\left(54 f^{3}-34 f^{2}+17 f-10\right) g-(f-1)^{2}\left(27 f^{2}-8 f+8\right) .
\end{array}
$$

It is a $\mathrm{K} 3$ surface of Picard number 19.

16.2. Analysis. The branch locus corresponds to the locus where the elliptic K3 surface acquires an extra $\mathrm{I}_{2}$ fiber. The transformation

$$
(f, g)=\left(\frac{2 x^{2} y+4 x y+y+x^{4}+x^{3}-3 x^{2}-x}{x^{3}(x+2)}, \frac{2 y+x^{2}-2 x-1}{x^{2}}\right)
$$

converts it to the elliptic curve

$$
y^{2}+y=x^{3}-x,
$$

which is 37a in Cremona's tables. This is an elliptic curve of rank 1, isomorphic to $X_{0}(37) /\langle w\rangle$, where $w$ is the Atkin-Lehner involution.

To analyze this Hilbert modular surface, note that we have a genus-1 fibration over $\mathbb{P}_{f}^{1}$, which has a section because the coefficient of $g^{4}$ is a square. Hence $Y_{-}$(37) is an elliptic K3 surface. Taking the Jacobian of this genus-1 curve over $\mathbb{Q}(f)$, and 


\begin{tabular}{l|c} 
Rational point $(f, g)$ & Sextic polynomial $f_{6}(x)$ defining the genus-2 curve $y^{2}=f_{6}(x)$ \\
\hline$\left(\frac{3}{2}, \frac{11}{7}\right)$ & $17 x^{6}-24 x^{5}-66 x^{4}+68 x^{3}+81 x^{2}-54 x-27$ \\
$\left(\frac{3}{4}, \frac{11}{9}\right)$ & $15 x^{6}-6 x^{5}-71 x^{4}+35 x^{3}+94 x^{2}-48 x-21$ \\
$\left(\frac{3}{2}, \frac{1}{3}\right)$ & $11 x^{6}-54 x^{5}-125 x^{4}-52 x^{3}-32 x^{2}-42 x-75$ \\
$\left(\frac{3}{2}, \frac{59}{65}\right)$ & $-135 x^{6}+108 x^{5}+45 x^{4}-44 x^{3}+130 x^{2}-12 x+95$ \\
$\left(\frac{1}{4}, \frac{17}{5}\right)$ & $81 x^{5}-135 x^{4}+13 x^{3}-9 x^{2}+70 x-15$ \\
$\left(-\frac{13}{3}, \frac{16}{13}\right)$ & $-13 x^{6}+156 x^{5}-24 x^{4}-132 x^{3}-45 x^{2}+108 x-27$ \\
$\left(-\frac{4}{9},-\frac{13}{6}\right)$ & $36 x^{6}-108 x^{5}+165 x^{4}-124 x^{3}+21 x^{2}+36 x-28$ \\
$\left(\frac{5}{3}, \frac{13}{10}\right)$ & $-54 x^{6}+54 x^{5}-9 x^{4}-84 x^{3}+141 x^{2}-180 x+100$ \\
$\left(\frac{3}{16}, \frac{13}{8}\right)$ & $52 x^{6}+156 x^{5}-39 x^{4}-180 x^{3}+9 x^{2}+72 x-16$ \\
$\left(-\frac{1}{2}, \frac{15}{13}\right)$ & $-31 x^{6}+156 x^{5}-195 x^{4}-260 x^{3}+210 x^{2}+156 x+23$ \\
$\left(3, \frac{81}{11}\right)$ & $-18 x^{6}+122 x^{5}-135 x^{4}-268 x^{3}-25 x^{2}+144 x+176$ \\
$\left(\frac{1}{4}, 3\right)$ & $x^{6}+72 x^{5}-18 x^{4}-189 x^{3}-117 x^{2}+270 x+45$ \\
$\left(\frac{31}{15}, \frac{16}{31}\right)$ & $-53 x^{6}+6 x^{5}-21 x^{4}+208 x^{3}-258 x^{2}-276 x+259$ \\
$\left(\frac{34}{27}, \frac{14}{51}\right)$ & $-2 x^{6}+36 x^{5}-138 x^{4}+105 x^{3}-33 x^{2}-153 x-289$ \\
$\left(3, \frac{7}{2}\right)$ & $-108 x^{6}-324 x^{5}-207 x^{4}-116 x^{3}+105 x^{2}-12 x-12$ \\
$\left(\frac{22}{3}, \frac{38}{11}\right)$ & $-22 x^{6}+72 x^{5}+84 x^{4}-341 x^{3}-441 x^{2}+417 x+473$ \\
&
\end{tabular}

Table 11. Some points of small height on the surface of Theorem 26 and the corresponding genus- 2 curves.

reparametrizing $f=t /(t+1)$, we get, after some Weierstrass transformations, the equation

$$
\begin{aligned}
y^{2}=x^{3}-(t+1)\left(27 t^{3}\right. & \left.+21 t^{2}-19 t-1\right) x^{2}-8 t^{2}(t+1)^{2}\left(30 t^{2}-235 t-1\right) x \\
& -16 t^{3}(t+1)^{2}\left(3136 t^{4}+5484 t^{3}+1024 t^{2}-2161 t-108\right) .
\end{aligned}
$$

This surface has reducible fibers of type IV at $t=-1$, type $\mathrm{I}_{2}$ at $t=-\frac{1}{28}$, and type $\mathrm{I}_{3}$ at $t=0, \infty$ and the four roots of $\left(27 t^{4}+45 t^{3}+10 t^{2}+22 t+3\right)$. This quartic polynomial describes a quadratic extension of $\mathbb{Q}(\sqrt{37})$. The bad fibers contribute $A_{2}^{7} \oplus A_{1}$ to the trivial lattice. We also have a 3-torsion section

$$
P_{0}=\left(4 t(t+1)\left(9 t^{2}+7 t-3\right), 4 t(t+1)\left(27 t^{4}+45 t^{3}+10 t^{2}+22 t+3\right)\right)
$$

and two non-torsion sections

$$
\begin{aligned}
P_{1}=\left(4 t(t+1)\left(49 t^{2}+28 t-1\right), 4 t(t+1)\left(637 t^{4}+854 t^{3}+276 t^{2}+33 t+1\right)\right), & \\
P_{2}=\left(4 \left(252 t^{4}+457 t^{3}+\right.\right. & \left.118 t^{2}-177 t-9\right) / 37, \\
& \left.4(t+3)(28 t+1)\left(27 t^{4}+45 t^{3}+10 t^{2}+22 t+3\right) / 37^{3 / 2}\right) .
\end{aligned}
$$

These two sections have height $\frac{8}{3}$ and $\frac{5}{6}$ respectively and are orthogonal with respect 
to the Néron-Tate height pairing. Therefore, the Néron-Severi lattice contains a sublattice of rank 19 and discriminant 1080. Counting points modulo 11 and 13 shows that the Picard number must be exactly 19. This is again confirmed by Oda's tables [1982, p. 109]. We checked that the Mordell-Weil subgroup generated by $P_{0}, P_{1}$ and $P_{2}$ is saturated at 2 and 3, and thus that we have the full Néron-Severi lattice.

16.3. Examples. Table 11 on the previous page lists some points of small height and their genus- 2 curves.

Next, we describe some curves of small genus on the surface, which may be used to produce rational points. The specialization $f=-\frac{1}{27}$ gives a rational curve, with parametrization $g=7\left(h^{2}-8 h+19\right) /\left(3\left(h^{2}-1\right)\right)$. The Brauer obstruction does not vanish identically for this rational curve.

The sections $P_{1}$ and $-P_{1}$ give rational curves, parametrized by

$$
g=\frac{13 f^{2}-7 f+3}{f(3 f+2)} \text { and } g=\frac{9 f^{2}-2 f+2}{7 f+1}
$$

respectively. The Brauer obstruction vanishes on both these loci, yielding families of genus- 2 curves whose Jacobians have real multiplication by $\mathcal{O}_{37}$.

\section{Discriminant 40}

17.1. Parametrization. We start with an elliptic $\mathrm{K} 3$ surface with fibers of type $E_{7}$, $D_{5}$ and $A_{4}$ at $t=\infty, 0$ and 1 respectively.

A Weierstrass equation for this family is given by

$$
\begin{aligned}
y^{2}=x^{3}+t\left(e^{2} t+(4 d+1)\right. & (1-t)) x^{2} \\
& +2 t^{2}(t-1)^{2}(2 d e t+2 d(d+1)(1-t)) x+4 d^{2} t^{3}(t-1)^{4}
\end{aligned}
$$

with

$$
d=(f-e+1)(f+e-1) / 2 .
$$

We first identify the class of a $D_{8}$ fiber, and perform a 2-neighbor step to an elliptic fibration with $D_{8}$ and $E_{7}$ fibers.

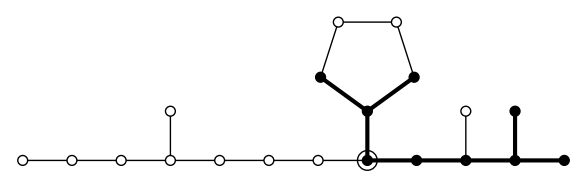

This fibration has a section $P$ of height $5=4+2-1$. Next, we take a 2-neighbor step to go to a fibration with $E_{8}$ and $E_{7}$ fibers. We have $P \cdot F^{\prime}=9$, while the intersection number of the $F^{\prime}$ with the remaining component of the $D_{8}$ fiber is 2 . 
Since these are coprime, the new elliptic fibration has a section.

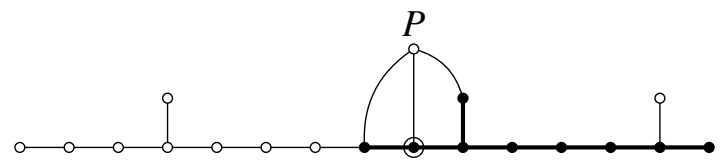

Finally, we read out the Igusa-Clebsch invariants, and compute the branch locus of the double cover, which is a union of two curves, one corresponding to an extra $\mathrm{I}_{2}$ fiber, the other to a promotion of the fiber at $t=\infty$ from $E_{7}$ to $E_{8}$.

Theorem 27. A birational model over $\mathbb{Q}$ for the Hilbert modular surface $Y_{-}(40)$ as a double cover of $\mathbb{P}_{e, f}^{2}$ is given by the equation

$z^{2}=-\left(f^{2}-e^{2}+1\right)\left(8 f^{4}+\left(-17 e^{2}+12 e-8\right) f^{2}+9 e^{4}-12 e^{3}+7 e^{2}+10 e+2\right)$.

It is a $\mathrm{K} 3$ surface of Picard number 19.

17.2. Analysis. The extra involution is $(e, f) \mapsto(e,-f)$. The branch locus is a union of two curves. The first, $f^{2}-e^{2}+1=0$, is a rational curve, parametrized by say $(e, f)=\left(\left(t^{2}+1\right) /(2 t),\left(t^{2}-1\right) /(2 t)\right)$. It corresponds to the subfamily of elliptic K3 surfaces for which the $E_{7}$ fiber is promoted to an $E_{8}$ fiber. The other component is also a rational curve, parametrized by

$$
(e, f)=\left(-\frac{\left(m^{2}-2\right)^{2}}{m(m-2)\left(m^{2}-4 m+2\right)}, \frac{2\left(m^{2}-2 m+2\right)\left(m^{2}-m-1\right)}{m(m-2)\left(m^{2}-4 m+2\right)}\right) .
$$

It corresponds to elliptic $\mathrm{K} 3$ surfaces with an extra $\mathrm{I}_{2}$ fiber.

The Hilbert modular surface is a double cover of a plane branched along a sextic, and is therefore a $\mathrm{K} 3$ surface. The transformation $f=e+t$ makes it a quartic in $e$, whose leading coefficient is a square. We thus get an elliptic K3 surface whose Weierstrass equation may be obtained by taking the Jacobian of this genus- 1 curve. After some elementary algebra, we get the elliptic K3 surface

$$
\begin{aligned}
y^{3}=x^{3}+\left(t^{4}+\right. & \left.24 t^{3}+98 t^{2}+16 t+1\right) x^{2} \\
+ & \left(128 t^{5}+2352 t^{4}+1088 t^{3}+64 t^{2}\right) x-\left(512 t^{6}+9216 t^{5}+704 t^{4}\right) .
\end{aligned}
$$

It has reducible fibers of types $\mathrm{I}_{6}$ at $t=\infty, \mathrm{I}_{4}$ at $t=0, \mathrm{I}_{3}$ at $t=-8 \pm \frac{5 \sqrt{10}}{2}$, and $\mathrm{I}_{2}$ at $t=\frac{1}{3}$ and $t=-9 \pm 4 \sqrt{5}$, giving a contribution of $A_{5} \oplus A_{3} \oplus A_{1}^{3} \oplus A_{2}^{2}$ to the Néron-Severi lattice. The trivial lattice thus has rank 17 and discriminant 1728.

We also have the two sections

$$
\begin{aligned}
& P_{1}=\left(4 t\left(2 t^{2}+36 t+3\right),-4 t\left(t^{2}+18 t+1\right)\left(2 t^{2}+32 t+3\right)\right), \\
& P_{2}=\left(-\left(6 t^{4}+124 t^{3}+303 t^{2}+138 t+9\right) / 10,\right. \\
& \left.\sqrt{10}(2 t+3)(3 t-1)\left(t^{2}+18 t+1\right)\left(2 t^{2}+32 t+3\right) / 100\right) .
\end{aligned}
$$


These have heights $\frac{1}{12}$ and $\frac{7}{6}$ respectively, and are orthogonal with respect to the height pairing. Therefore, the Picard number is at least 19. Counting points modulo 7 and 11 proves that the Picard number is 19 (we thank Ronald van Luijk for carrying out such a calculation), in agreement with Oda's calculations [1982, p. 109]. The part of the Néron-Severi lattice spanned by the above sections with the trivial lattice has rank 19 and discriminant 168 . We showed that this lattice is 2-saturated, so it is the full Néron-Severi lattice.

The quotient of the Hilbert modular surface by the involution $f \mapsto-f$ is the rational elliptic surface

$$
y^{2}=\left(x+8 e^{2}-8\right)\left(x^{2}+\left(17 e^{2}-12 e+8\right) x+8(3 e+1)^{2}\left(e^{2}-2 e+2\right)\right) .
$$

It has reducible fibers of types $\mathrm{I}_{6}, \mathrm{I}_{3}, \mathrm{I}_{2}$ at $e=\infty, 8,9$, respectively, and the MordellWeil group is generated by the 6-torsion section

$$
\left(-4\left(2 e^{2}+e-11\right), 4(e-9)(e-8)\right)
$$

indeed this is the universal elliptic curve over $X_{1}(6)$.

17.3. Examples. Table 12 lists some points of small height and their genus-2 curves.

Rational point $(e, f) \quad$ Sextic polynomial $f_{6}(x)$ defining the genus-2 curve $y^{2}=f_{6}(x)$

\begin{tabular}{l|c}
\hline$\left(\frac{49}{8},-\frac{47}{8}\right)$ & $-12 x^{6}-12 x^{5}-21 x^{4}+14 x^{3}+39 x^{2}+48 x-64$ \\
$\left(\frac{31}{6}, 5\right)$ & $108 x^{6}+108 x^{5}-81 x^{4}+x^{3}+63 x^{2}-33 x+9$ \\
$\left(-\frac{1}{12},-\frac{5}{6}\right)$ & $36 x^{5}+78 x^{4}-41 x^{3}-129 x^{2}+45 x+27$ \\
$\left(-\frac{13}{10},-\frac{4}{5}\right)$ & $-72 x^{6}+108 x^{5}+135 x^{4}-135 x^{3}-219 x^{2}+135 x+53$ \\
$\left(\frac{49}{8}, \frac{47}{8}\right)$ & $-72 x^{6}+216 x^{5}-315 x^{4}+162 x^{3}+21 x^{2}-72 x-16$ \\
$\left(-\frac{23}{14},-\frac{13}{7}\right)$ & $-8 x^{6}+60 x^{5}-87 x^{4}-163 x^{3}+288 x^{2}+324 x+27$ \\
$\left(-\frac{29}{12},-\frac{25}{12}\right)$ & $12 x^{6}+132 x^{5}+355 x^{4}-90 x^{3}-245 x^{2}+36 x-44$ \\
$\left(\frac{87}{55}, \frac{12}{55}\right)$ & $44 x^{5}+200 x^{4}-422 x^{3}+180 x^{2}-81 x$ \\
$\left(-\frac{75}{28}, \frac{18}{7}\right)$ & $-77 x^{6}+147 x^{5}-45 x^{4}-335 x^{3}+186 x^{2}-180 x-432$ \\
$\left(\frac{49}{23}, \frac{43}{23}\right)$ & $46 x^{6}-24 x^{5}+252 x^{4}-29 x^{3}+468 x^{2}+24 x+366$ \\
$\left(-\frac{37}{36}, \frac{35}{36}\right)$ & $-18 x^{6}-258 x^{5}-475 x^{4}+220 x^{3}-325 x^{2}+72 x-48$ \\
$\left(\frac{19}{8}, \frac{13}{8}\right)$ & $432 x^{6}+216 x^{5}-27 x^{4}-502 x^{3}-87 x^{2}+36 x+116$ \\
$\left(\frac{55}{28},-\frac{43}{28}\right)$ & $-496 x^{6}+48 x^{5}-545 x^{4}+90 x^{3}-257 x^{2}+120 x-80$ \\
$\left(-\frac{1}{28}, \frac{15}{28}\right)$ & $314 x^{6}+426 x^{5}+555 x^{4}+140 x^{3}-195 x^{2}-264 x-176$ \\
$\left(-\frac{23}{15}, \frac{22}{15}\right)$ & $-586 x^{6}+330 x^{5}-512 x^{4}+150 x^{3}-110 x^{2}-24 x-1$ \\
$\left(-\frac{5}{4},-\frac{1}{4}\right)$ & $8 x^{6}-168 x^{5}-269 x^{4}+466 x^{3}+451 x^{2}-624 x-376$ \\
\hline
\end{tabular}

Table 12. Some points of small height on the surface of Theorem 27 and the corresponding genus- 2 curves. 
The section $P_{1}$ gives a rational curve

$$
(e, f)=\left(-\frac{2 g^{2}+11}{4 g-1}, \frac{2 g^{2}-g-11}{4 g-1}\right) .
$$

However, the Brauer obstruction does not vanish identically on this locus. The section $2 P_{1}$ gives a rational curve

$$
(e, f)=\left(-\frac{2 g^{2}+3}{4 g}, \frac{2 g^{2}-3}{4 g}\right)
$$

on the surface. Here, the Brauer obstruction does vanish identically, yielding a family of genus- 2 curves with real multiplication by $\mathcal{O}_{40}$.

\section{Discriminant 41}

18.1. Parametrization. We start with an elliptic $\mathrm{K} 3$ surface with fibers of type $A_{5}$ at $t=0$ and $A_{10}$ at $t=\infty$, with a section of height $\frac{41}{66}=4-\frac{4 \cdot 7}{11}-\frac{5}{6}$.

A Weierstrass equation for this family is given by

$$
y^{2}=x^{3}+\left(t^{2}+2 d f t+c f^{2}\right) x^{2}+2 t^{2}(d t+c f) x+c t^{4},
$$

with

$$
\begin{aligned}
& c=r^{2} s^{2}\left(16 t^{2}-8(4 r s-16 s-r) t+(4 r s-16 s+r)^{2}\right), \\
& d=r s(4 t-12 r s+16 s+r), \\
& f=(t+4 s) /(4 r s) .
\end{aligned}
$$

We identify the class of an $E_{8}$ fiber, and perform a 3-neighbor step to an elliptic fibration with $E_{8}$ and $A_{7}$ fibers.

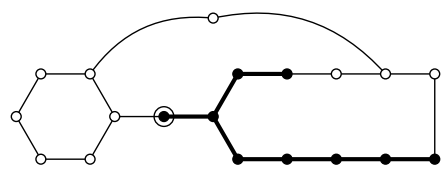

This fibration has a section $P$ of height $\frac{41}{8}=4+2-\frac{7}{8}$. Next, we take a 2-neighbor step to go to a fibration with $E_{8}$ and $E_{7}$ fibers.

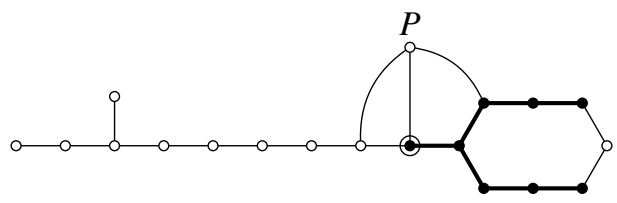

The intersection number of the new fiber $F^{\prime}$ with the remaining component of the $A_{7}$ fiber is 2 and with the section $P$ is 5 . Since these number are coprime, the new genus-1 fibration defined by $F^{\prime}$ has a section. 
Reading out the map to $\mathcal{M}_{2}$ from the $E_{8} E_{7}$ fibration, we obtain the following result.

Theorem 28. A birational model over $\mathbb{Q}$ for the Hilbert modular surface $Y_{-}(41)$ as a double cover of $\mathbb{P}_{r, s}^{2}$ is given by the equation

$$
\begin{aligned}
z^{2}=(4 s+1)^{4} r^{4}- & 16\left(8 s^{2}+42 s-1\right)(4 s+1)^{2} r^{3} \\
+ & 32\left(128 s^{4}+4672 s^{3}+1976 s^{2}+248 s+3\right) r^{2} \\
- & 256\left(2688 s^{3}+872 s^{2}+82 s-1\right) r+256(16 s+1)^{3} .
\end{aligned}
$$

It is a $\mathrm{K} 3$ surface of Picard number 19.

18.2. Analysis. The branch locus is a rational curve, with a parametrization given by

$$
(r, s)=\left(-\frac{4(u-1)(u+1)^{3}}{\left(u^{2}-u-1\right)^{2}},-\frac{1}{4 u^{2}(u-1)^{2}}\right) .
$$

The Hilbert modular surface has a genus-1 fibration to $\mathbb{P}_{r}^{1}$, which is in fact an elliptic fibration, since the coefficient of $r^{4}$ is a perfect square. Converting to the Jacobian form, we get the Weierstrass equation

$$
\begin{aligned}
y^{2}=x^{3}+(t-1)\left(t^{3}+23 t^{2}+96 t\right. & -32) x^{2} \\
& +16(t-1)\left(4 t^{4}+53 t^{3}-217 t^{2}+112 t-16\right) x .
\end{aligned}
$$

This is an elliptic K3 surface, with bad fibers of type $\mathrm{I}_{6}$ at $t=\infty, \mathrm{I}_{4}$ at $t=0$, III at $t=1, \mathrm{I}_{3}$ at $t=-15$, and $\mathrm{I}_{2}$ at the four roots of $q(t)=4 t^{4}+53 t^{3}-217 t^{2}+112 t-16$. This quartic $q(t)$ describes a dihedral Galois extension $K$ of $\mathbb{Q}$, quadratic over $\mathbb{Q}(\sqrt{41})$. Thus we get a trivial lattice of rank 17 and discriminant 2304 . We also have a 2 -torsion point $(0,0)$ and the two non-torsion sections

$$
\begin{aligned}
P_{1}= & (-164(t-1), 4 \mu(t-1)(t+15)(5 t-6)), \\
P_{2}= & \left((5+\mu)(t-1)\left(8 t^{2}+53 t+9 t \mu-185-29 \mu\right) / 16,\right. \\
& \left.\quad(5+\mu)(t-1)(t+15)(4 t-11+\mu)\left(8 t^{2}+53 t+9 t \mu-185-29 \mu\right) / 64\right),
\end{aligned}
$$

where $\mu=\sqrt{41}$. The height matrix for $P_{1}$ and $P_{2}$ is

$$
\frac{1}{3}\left(\begin{array}{rr}
4 & -2 \\
-2 & 1
\end{array}\right) \text {. }
$$

Therefore the Picard number is at least 19. Counting points modulo 7 and 11 shows that the Picard number must be 19, in agreement with [Oda 1982]. The sections above and the trivial lattice therefore generate a lattice of rank 19 and discriminant 512. We showed that it is 2-saturated, and is thus the full Néron-Severi lattice. 


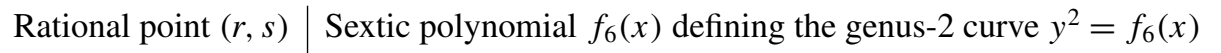

\begin{tabular}{lc}
$\left(\frac{52}{3},-\frac{1}{5}\right)$ & $-6 x^{6}+3 x^{5}-4 x^{4}+3 x^{3}+20 x^{2}-36 x+20$ \\
$\left(\frac{7}{2}, \frac{13}{12}\right)$ & $-x^{6}-15 x^{5}-65 x^{4}-57 x^{3}+40 x^{2}+6 x+20$ \\
$\left(\frac{56}{9},-\frac{43}{40}\right)$ & $-56 x^{6}-42 x^{5}+64 x^{4}-78 x^{3}-29 x^{2}+36 x-28$ \\
$\left(\frac{52}{7},-\frac{2}{3}\right)$ & $-24 x^{6}+36 x^{5}-34 x^{4}-51 x^{3}+86 x^{2}-87 x+18$ \\
$\left(13,-\frac{7}{12}\right)$ & $-15 x^{6}+33 x^{5}+23 x^{4}+73 x^{3}-72 x^{2}-54 x-108$ \\
$\left(\frac{32}{5},-\frac{47}{48}\right)$ & $-18 x^{6}-3 x^{5}+46 x^{4}-111 x^{3}+22 x^{2}+48 x-84$ \\
$\left(\frac{92}{9},-\frac{11}{14}\right)$ & $-4 x^{6}+24 x^{5}-17 x^{4}+3 x^{3}-125 x^{2}-72 x-95$ \\
$\left(\frac{60}{13}, 3\right)$ & $-10 x^{6}+33 x^{5}+40 x^{4}-111 x^{3}-152 x^{2}+60 x+140$ \\
$\left(\frac{76}{13},-\frac{4}{3}\right)$ & $-24 x^{6}-12 x^{5}-50 x^{4}+111 x^{3}+10 x^{2}+129 x-153$ \\
$\left(28,-\frac{23}{24}\right)$ & $-46 x^{6}+69 x^{5}-14 x^{4}+169 x^{3}-134 x^{2}-84 x-72$ \\
$\left(\frac{68}{15},-\frac{11}{4}\right)$ & $48 x^{6}-48 x^{5}-119 x^{4}-84 x^{3}+145 x^{2}+180 x+100$ \\
$\left(\frac{23}{6}, \frac{21}{4}\right)$ & $168 x^{5}+85 x^{4}+70 x^{3}+229 x^{2}+24 x-72$ \\
$\left(\frac{40}{3},-\frac{55}{56}\right)$ & $-39 x^{6}+36 x^{5}-116 x^{4}+186 x^{3}-107 x^{2}+240 x-200$ \\
$\left(\frac{52}{25},-\frac{1}{24}\right)$ & $-112 x^{6}-264 x^{5}+25 x^{4}+240 x^{3}-125 x^{2}-114 x+62$ \\
$\left(\frac{4}{13},-\frac{11}{96}\right)$ & $16 x^{6}+24 x^{5}-223 x^{4}+274 x^{3}-7 x^{2}+216 x-120$ \\
$\left(\frac{27}{2}, \frac{11}{76}\right)$ & $12 x^{6}-132 x^{5}-219 x^{4}+286 x^{3}+201 x^{2}-264 x-56$ \\
\hline
\end{tabular}

Table 13. Some points of small height on the surface of Theorem 28 and the corresponding genus- 2 curves.

18.3. Examples. Table 13 lists some points of small height and their genus-2 curves.

Next, we describe some curves which are a source of many rational points. The specialization $s=-4$ gives a curve of genus 0 , with a parametrization $r=$ $-2\left(m^{2}+343\right) /(9(m-13))$. The specialization $r=\frac{108}{25}$ also gives a rational curve, parametrized by $s=-(4 m-169)(4 m+169) /(16(108 m-10813))$. The Brauer obstruction vanishes on both these loci, giving families of curves whose Jacobians have real multiplication by $\mathcal{O}_{41}$.

Finally, sections of the elliptic fibration also give rational curves on the surface. For instance, the section $P_{0}$ is parametrized by

$$
r=\frac{4\left(256 s^{3}-48 s^{2}-16 s-1\right)}{(4 s+1)^{2}(16 s-7)}
$$

The Brauer obstruction vanishes here as well.

\section{Discriminant 44}

19.1. Parametrization. We start with an elliptic $\mathrm{K} 3$ surface with fibers of type $A_{10}$ at $t=0$ and $D_{6}$ at $t=\infty$. This has Néron-Severi lattice of discriminant $11 \cdot 4=44$ 
and rank $2+10+6=18$. The Weierstrass equation for this universal family can be written as

$$
y^{2}=x^{3}+a x^{2}+2 b t^{4} x+c t^{8}
$$

with

$$
\begin{aligned}
& a=s\left(r^{2} s^{2}-s^{2}+2 s+2\right) t^{3}-s\left(2 r^{2} s-3 s+2\right) t^{2}+s\left(r^{2}-3\right) t+1, \\
& b=s^{2}\left(\left(2 r^{2} s^{2}-2 s^{2}+4 s+1\right) / 2 t^{2}-\left(r^{2} s-2 s+1\right) t-1\right) \\
& c=s^{4}\left(\left(r^{2} s-s+2\right) t+1\right)
\end{aligned}
$$

We identify the class of an $E_{8}$ fiber and move to this fibration via a 3-neighbor step.

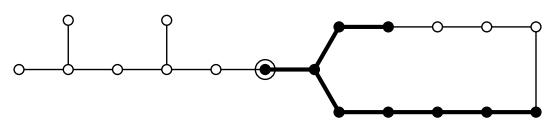

The new elliptic fibration has $E_{8}, D_{6}$ and $A_{1}$ fibers, and a section $P$ of height $\frac{11}{2}=4+2 \cdot 1-\frac{1}{2}$. We identify an $E_{7}$ fiber $F^{\prime}$ below. Note that $P \cdot F^{\prime}=5$, while the excluded component of the $D_{6}$ fiber intersects $F^{\prime}$ in 2. Since 2 and 5 are coprime, we see that the fibration defined by $F^{\prime}$ has a section. We move to it by a 2-neighbor step.

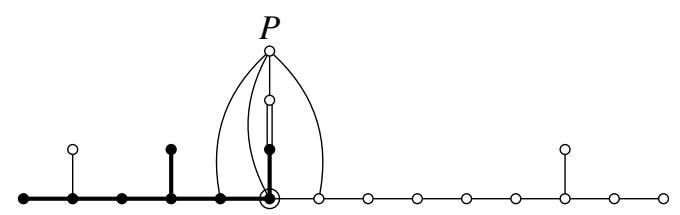

Calculating the Igusa-Clebsch invariants and following the rest of the algorithm in Section 4, we obtain the following result.

Theorem 29. A birational model over $\mathbb{Q}$ for the Hilbert modular surface $Y_{-}(44)$ as a double cover of $\mathbb{P}_{r, s}^{2}$ is given by the equation

$$
z^{2}=(r s+s-1)(r s-s+1)\left(r^{6} s^{2}-r^{4} s^{2}+18 r^{2} s-16 s+27\right) .
$$

It is an honestly elliptic surface, with arithmetic genus 2 and Picard number 29.

19.2. Analysis. The extra involution is $\iota:(r, s) \mapsto(-r, s)$. The branch locus has three components. The two simpler components $r s \pm(s-1)=0$ are obviously rational curves, and a simple calculation shows that they correspond to elliptic K3 surfaces for which the $D_{6}$ fiber gets promoted to an $E_{7}$ fiber. The more complicated component of the branch locus, corresponding to elliptic fibrations with an extra $\mathrm{I}_{2}$ fiber, is also a rational curve; a parametrization is given by

$$
(r, s)=\left(\frac{4 m}{m^{2}+3},-\frac{\left(m^{2}+3\right)^{3}}{16\left(m^{2}-1\right)}\right) .
$$


The right-hand side of the equation defining the Hilbert modular surface is quartic in $s$, whence the surface $Y_{-}(44)$ is elliptically fibered over $\mathbb{P}_{r}^{1}$. The two linear factors (or the fact that the coefficient of $s^{4}$ is a square) imply that there are sections, so we may convert to the Jacobian form:

$$
y^{2}=x^{3}+2\left(r^{6}-r^{4}-9 r^{2}+11\right) x^{2}+\left(r^{2}-1\right)^{3}\left(r^{6}+r^{4}+91 r^{2}-121\right) x .
$$

This is an honestly elliptic surface, with $\chi=3$. It has reducible fibers of type $\mathrm{I}_{2}$ at $r=0, \mathrm{I}_{6}$ at $r= \pm 1, \mathrm{I}_{4}$ at $r=\infty, \mathrm{I}_{3}$ at $r= \pm \frac{2}{\sqrt{3}}$, and type $\mathrm{I}_{2}$ at the six roots of $\left(r^{3}-3 r^{2}+5 r+11\right)\left(r^{3}+3 r^{2}+5 r-11\right)$ (both factors generate the cubic field $k_{-44}$ of discriminant -44). The trivial lattice has rank 26, leaving room for Mordell-Weil rank at most 4 . We find the following sections, of which $P_{0}$ is 2-torsion, while $P_{1}$, $P_{2}$ and $P_{3}$ are linearly independent non-torsion sections, orthogonal with respect to the height pairing and of heights $\frac{7}{6}, \frac{3}{2}$ and $\frac{11}{6}$ respectively:

$$
\begin{aligned}
& P_{0}=(0,0), \\
& P_{1}=\left(11\left(r^{2}-1\right), 4 \sqrt{11} r\left(r^{2}-1\right)^{3}\left(3 r^{2}-4\right)\right), \\
& P_{2}=\left(-(r+1)^{3}\left(r^{3}-3 r^{2}+5 r+11\right), 6 \sqrt{-3} r(r+1)^{3}\left(r^{3}-3 r^{2}+5 r+11\right)\right), \\
& P_{3}=\left((r-1)(r+1)^{2}\left(r^{3}-3 r^{2}+5 r+11\right), 2 r^{3}(r-1)(r+1)^{2}\left(r^{3}-3 r^{2}+5 r+11\right)\right) .
\end{aligned}
$$

Therefore, the Picard number is at least 29. Analysis of the associated quotient elliptic K3 surface and its twist (see below) shows that the Mordell-Weil rank is exactly 3 and therefore the Picard number is exactly 29 . The sections above together with the trivial lattice generate a lattice of discriminant $133056=2^{6} \cdot 3^{3} \cdot 7 \cdot 11$. We checked that it is 2- and 3-saturated, and so it is the entire Néron-Severi lattice. Therefore these sections generate the Mordell-Weil group.

We next analyze the quotient of $Y_{-}(44)$ by the involution $\iota$. Taking $t=r^{2}$, we find the equation

$$
\begin{aligned}
z^{2}=s^{4} t^{4}-s^{2}\left(2 s^{2}-2 s+1\right) t^{3}+s^{2}\left(s^{2}+16 s+1\right) t^{2} \\
-s(2 s-3)(17 s-6) t+(s-1)^{2}(16 s-27) .
\end{aligned}
$$

This has an elliptic fibration over $\mathbb{P}_{s}^{1}$, and since the coefficient of $t^{4}$ is a square, we may convert to the Jacobian, which is

$$
y^{2}=x^{3}+s^{2}(s+2)(s+8) x^{2}+2 s^{3}\left(6 s^{2}+47 s+9\right) x+s^{4}\left(36 s^{2}+268 s-27\right) .
$$

This is an elliptic K3 surface, with reducible fibers of type $E_{6}$ at $s=0, \mathrm{I}_{7}$ at $s=\infty$, $\mathrm{I}_{3}$ at $s=-\frac{27}{4}$, and $\mathrm{I}_{2}$ at the roots of $2 s^{3}+14 s^{2}-6 s+1$, which generates the cubic field $k_{-44}$. The trivial lattice has rank 19 . We find a non-torsion section

$$
P=\left(1-4 s, 2 s^{3}+14 s^{2}-6 s+1\right)
$$


of height $4-\frac{12}{7}-\frac{3}{2}=\frac{11}{14}$. It is easy to show that $P$ generates the Mordell-Weil group: the configuration of reducible fibers does not allow for either nontrivial torsion or a section of height $11 / 14 n^{2}$ for any integer $n>1$. Therefore the K3 surface is singular, with Néron-Severi lattice of discriminant $-396=-4 \cdot 9 \cdot 11$.

We may also analyze the quotient by considering it as an elliptic surface over $\mathbb{P}_{t}^{1}$, and since the coefficient of $s^{4}$ is $t^{2}(t-1)^{2}$, which is a square, there is a section. Converting to the Jacobian, we get the elliptic K3 surface

$$
y^{2}=x^{3}+2\left(t^{3}-t^{2}-9 t+11\right) x^{2}+(t-1)^{3}\left(t^{3}+t^{2}+91 t-121\right) x
$$

which is also obtained by replacing $r^{2}$ by $t$ in the Weierstrass equation of $Y_{-}(44)$. This elliptic fibration has bad fibers of type $\mathrm{I}_{6}^{*}, \mathrm{I}_{6}$ and $\mathrm{I}_{3}$ at $t=\infty, 1$ and $\frac{4}{3}$ respectively, and of type $\mathrm{I}_{2}$ at the roots of $t^{3}+t^{2}+91 t-121$, which generates $k_{-44}$. Therefore, the root lattice has rank 18 . We find the sections

$$
\begin{aligned}
& P_{0}=(0,0), \\
& P_{1}=\left((1-t)(t-3 \sqrt{-3})^{2}, 2(3-\sqrt{-3})(t-1)(t-3 \sqrt{-3})(9 t-6-2 \sqrt{-3}) / 3\right) \\
& P_{2}=\left((1-t)(t+3 \sqrt{-3})^{2}, 2(3+\sqrt{-3})(t-1)(t+3 \sqrt{-3})(9 t-6+2 \sqrt{-3}) / 3\right),
\end{aligned}
$$

with $P_{0}$ being 2-torsion, and $P_{1}$ and $P_{2}$ having height pairing matrix

$$
\left(\begin{array}{ll}
\frac{5}{3} & \frac{1}{6} \\
\frac{1}{6} & \frac{5}{3}
\end{array}\right) .
$$

These sections, along with the trivial lattice, generate a lattice of rank 20 and discriminant -396 , which must therefore be the entire Néron-Severi lattice. The Mordell-Weil rank of this elliptic surface is 2.

Finally, we consider the quadratic twist of the above elliptic $\mathrm{K} 3$ surface, which is the quotient of $Y_{-}(44)$ by the involution $\iota^{\prime}:(r, s, z) \mapsto(-r, s,-z)$. It is given by the equation

$$
y^{2}=x^{3}+2 t\left(t^{3}-t^{2}-9 t+11\right) x^{2}+t^{2}(t-1)^{3}\left(t^{3}+t^{2}+91 t-121\right) x .
$$

This is an elliptic K3 surface with reducible fibers of type $\mathrm{I}_{1}^{*}, \mathrm{I}_{6}, \mathrm{I}_{3}$ and $\mathrm{I}_{2}$ at $t=0$, $1, \frac{4}{3}$ and $\infty$ respectively, and $\mathrm{I}_{2}$ at the roots of $t^{3}+t^{2}+91 t-121$. The trivial lattice has rank 18 . We find the sections

$$
\begin{aligned}
& P_{0}=(0,0), \\
& P_{1}=\left(11 t(t-1)^{3}, 4 \sqrt{11} t^{2}(t-1)^{3}(3 t-4)\right),
\end{aligned}
$$

the first being 2-torsion, and the second of height $\frac{7}{12}$. The Picard number is therefore at least 19. Counting points modulo 5 and 7 shows that it is exactly 19; therefore the Mordell-Weil rank is exactly 1 . These sections and the trivial lattice span 
a sublattice of discriminant 168 of the Néron-Severi lattice. This sublattice is 2 -saturated, since the configuration of fibers does not allow for a section of height $\frac{7}{48}$, and we can easily check that the elliptic surface does not have 4-torsion or other 2-torsion sections. Therefore, we have the entire Néron-Severi lattice, and $P_{0}$ and $P_{1}$ generate the Mordell-Weil group.

The calculation of the Mordell-Weil ranks of the quotient elliptic surface and its quadratic twist allows us to conclude that the Mordell-Weil rank of the original (honestly) elliptic surface is $1+2=3$.

19.3. Examples. Table 14 lists some points of small height and their genus-2 curves.

The specializations $r= \pm 1$ give rational curves on the surface, but points on these correspond to decomposable abelian surfaces, which therefore have an endomorphism ring strictly larger than $\mathcal{O}_{44}$. The section $P_{0}$ is a rational curve, given by $s=\left(r^{4}+27\right) /\left(2\left(r^{2}-1\right)\left(r^{2}-8\right)\right)$. However, the Brauer obstruction does not vanish identically on it.

\begin{tabular}{l|c}
$(r, s)$ & Sextic polynomial $f_{6}(x)$ defining the genus-2 curve $y^{2}=f_{6}(x)$ \\
\hline$\left(-2,-\frac{7}{6}\right)$ & $101 x^{6}-60 x^{5}+2 x^{4}+92 x^{2}+48 x+24$ \\
$\left(2,-\frac{7}{6}\right)$ & $15 x^{6}-168 x^{5}+170 x^{4}-112 x^{3}+20 x^{2}-8$ \\
$\left(4,-\frac{7}{15}\right)$ & $-24 x^{6}+48 x^{5}+52 x^{4}+144 x^{3}+238 x^{2}+588 x-161$ \\
$\left(-4,-\frac{7}{15}\right)$ & $56 x^{6}+196 x^{4}-320 x^{3}+250 x^{2}-480 x+723$ \\
$\left(-5,-\frac{13}{12}\right)$ & $-144 x^{6}+120 x^{5}+265 x^{4}+700 x^{3}-425 x^{2}-750 x-1750$ \\
$\left(2, \frac{7}{12}\right)$ & $696 x^{6}-2112 x^{5}+7492 x^{4}-7032 x^{3}+10234 x^{2}-756 x+5103$ \\
$\left(5,-\frac{13}{12}\right)$ & $12 x^{6}-60 x^{5}-145 x^{4}+400 x^{3}-1225 x^{2}-1500 x+11500$ \\
$\left(2, \frac{13}{36}\right)$ & $-5193 x^{6}-5124 x^{5}-16906 x^{4}-11576 x^{3}-17212 x^{2}-6240 x-5304$ \\
$\left(-\frac{3}{4}, \frac{36}{7}\right)$ & $-4744 x^{6}-15552 x^{5}+7596 x^{4}+42048 x^{3}-20310 x^{2}-32112 x+33553$ \\
$\left(-2, \frac{13}{36}\right)$ & $-18261 x^{6}+13668 x^{5}+65210 x^{4}-41512 x^{3}-74284 x^{2}+18816 x+24696$ \\
$\left(\frac{3}{4}, \frac{36}{7}\right)$ & $-6504 x^{6}+22608 x^{5}+28428 x^{4}-94288 x^{3}-66510 x^{2}+103092 x+73009$ \\
$\left(-2, \frac{7}{12}\right)$ & $25389 x^{6}-97062 x^{5}-511 x^{4}+240860 x^{3}-5989 x^{2}-127758 x-83849$ \\
$\left(-5,-\frac{9}{32}\right)$ & $-691156 x^{6}+20220 x^{5}-232521 x^{4}+19406 x^{3}-22521 x^{2}+2484 x-564$ \\
$\left(2,-\frac{61}{42}\right)$ & $-629624 x^{6}+272400 x^{5}-383596 x^{4}-704000 x^{3}-60778 x^{2}$ \\
$\left(-2,-\frac{61}{42}\right)$ & $-550872 x^{6}+1549296 x^{5}-1810124 x^{4}-2005984 x^{3}$ \\
$\left(5,-\frac{9}{32}\right)$ & $1781676 x^{6}-5240052 x^{5}+5462991 x^{4}-5705734 x^{3}$ \\
& $+1769571 x^{2}+1002576 x-1011776$ \\
\hline
\end{tabular}

Table 14. Some rational points $(r, s)$ of small height on the surface of Theorem 29 and the corresponding genus- 2 curves. 


\section{Discriminant 53}

20.1. Parametrization. We start with an elliptic $\mathrm{K} 3$ surface with $A_{8}, A_{6}$ and $A_{1}$ fibers, and a section of height $\frac{53}{126}=4-\frac{1}{2}-\frac{6}{7}-\frac{20}{9}$. A Weierstrass equation for this family is given by

$$
y^{2}=x^{3}+a x^{2}+2 b t(t-h) x+c t^{2}(t-h)^{2},
$$

with

$$
\begin{aligned}
& \begin{aligned}
& a= g^{2} t^{4}+\left(4(h+1)^{2}-2 g\right) t^{3}+\left(4 h^{2}+4 g h+6 g-3\right) t^{2} \\
&+2\left(8 h^{2}-4 g h+8 h+1\right) t+(2 h+1)^{2}, \\
& b=-4(h-g+1)\left(g(2 h+1) t^{2}+\left(6 h^{2}-2 g h+6 h+1\right) t+(2 h+1)^{2}\right), \\
& c=16(h-g+1)^{2}(2 h+1)^{2} .
\end{aligned}
\end{aligned}
$$

To transform to a fibration with $E_{8}$ and $E_{7}$ fibers, we first identify an $E_{7}$ fiber, and move to the associated elliptic fibration via a 2-neighbor step.

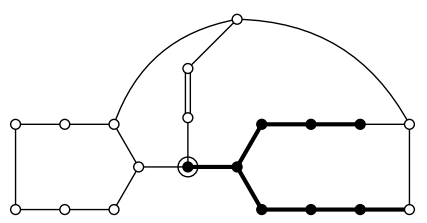

The resulting elliptic fibration has $E_{7}$ and $A_{8}$ fibers, and a section $P$ of height $\frac{53}{18}=4+2 \cdot 1-\frac{3}{2}-4 \cdot \frac{5}{9}$. We identify a fiber $F$ of type $E_{8}$ and perform a 3-neighbor step to move to the associated elliptic fibration. Note that $P \cdot F=4$, while the remaining component of the $A_{8}$ fiber intersects $F$ with multiplicity 3 . Therefore the new elliptic fibration has a section.

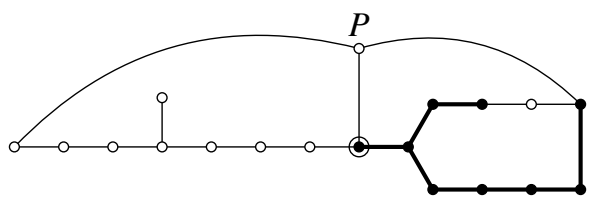

The new elliptic fibration has the requisite $E_{8}$ and $E_{7}$ fibers, and therefore we may read out the Igusa-Clebsch invariants, and describe the branch locus, which corresponds to elliptic $\mathrm{K} 3$ surfaces having an extra $\mathrm{I}_{2}$ fiber.

Theorem 30. A birational model over $\mathbb{Q}$ for the Hilbert modular surface $Y_{-}(53)$ as a double cover of $\mathbb{P}_{g, h}^{2}$ is given by the equation

$$
\begin{aligned}
z^{2}=- & 27 h^{4} g^{4}-2 h^{3}\left(13 h^{2}+9 h+9\right) g^{3} \\
& -\left(11 h^{6}+138 h^{5}+383 h^{4}+506 h^{3}+353 h^{2}+120 h+16\right) g^{2} \\
& -2(h+1)^{2}\left(52 h^{4}+99 h^{3}+65 h^{2}+19 h+2\right) g-(h+1)^{4}\left(44 h^{3}+76 h^{2}+40 h+7\right) .
\end{aligned}
$$


It is an honestly elliptic surface, with arithmetic genus 2 and Picard number 28.

20.2. Analysis. The branch locus is a curve of genus 1 , and the change of coordinates

$$
\begin{aligned}
& g=-\frac{\left(x^{2}-x+1\right)\left(x^{2}+3 x+3\right)}{(x+1)(2 x+1) y+x^{4}+3 x^{3}+5 x^{2}+3}, \\
& h=\frac{\left(x^{5}-x^{4}+4 x^{3}-4 x^{2}+x+1\right) y+x^{2}\left(x^{6}-x^{5}+2 x^{4}+2 x^{3}-x+1\right)}{\left(x^{2}-x+1\right)^{2}\left((x+1)(2 x+1) y+x^{4}+3 x^{3}+5 x^{2}+3\right)}
\end{aligned}
$$

transforms it to the elliptic curve $y^{2}+x y+y=x^{3}-x^{2}$ of conductor 53, which is isomorphic to $X_{0}(53) /\langle w\rangle$, where $w$ is the Atkin-Lehner involution.

This surface has a genus- 1 fibration over $\mathbb{P}_{h}^{1}$, making it honestly elliptic with $\chi=3$. We could find no sections over $\mathbb{Q}$, although there certainly exist sections over $\overline{\mathbb{Q}}$, since the coefficient of $g^{4}$ is a square in $\mathbb{Q}(\sqrt{-3})$. The Jacobian of this fibration has the following equation (after making the linear fractional transformation $h=-t /(t+1)$ on the base, and some Weierstrass transformations):

$$
\begin{aligned}
& y^{2}=x^{3}+\left(t^{6}-18 t^{5}+55 t^{4}+106 t^{3}-179 t^{2}+24 t-16\right) x^{2} \\
&-8(t-1) t^{2}\left(37 t^{5}-471 t^{4}-140 t^{3}+1121 t^{2}-309 t+248\right) x \\
& \quad-16(t-1)^{2} t^{4}\left(196 t^{5}-2797 t^{4}+2712 t^{3}+8606 t^{2}-3084 t+3115\right) .
\end{aligned}
$$

This surface has reducible fibers of type $\mathrm{I}_{7}, \mathrm{I}_{4}$ and $\mathrm{I}_{3}$ at $t=\infty, 0,1$ respectively, type $\mathrm{I}_{2}$ at the roots of $7 t^{3}-99 t^{2}+104 t-32$ (which generates the cubic field of discriminant $-2^{2} \cdot 53$ ), and $\mathrm{I}_{3}$ at the roots of $t^{5}-11 t^{4}-11 t^{3}+6 t^{2}-3 t-9$ (which generates the quintic field of discriminant $-3^{2} \cdot 53^{2}$, and whose roots generate a dihedral $D_{10}$ extension unramified over its quadratic subfield $\mathbb{Q}(\sqrt{-3 \cdot 53})$ ). The trivial lattice has rank 26, leaving room for Mordell-Weil rank at most 4 . We find the sections

$$
\begin{aligned}
& P_{1}=\left(-4\left(7 t^{6}-106 t^{5}+189 t^{4}+202 t^{3}-778 t^{2}+342 t-216\right) / 53,\right. \\
&\left.4(5 t-3)\left(7 t^{3}-99 t^{2}+104 t-32\right)\left(t^{5}-11 t^{4}-11 t^{3}+6 t^{2}-3 t-9\right) /(53 \sqrt{53})\right) \\
& P_{2}=\left(-4\left(49 t^{6}-63 t^{5}+99 t^{4}+162 t^{3}-351 t^{2}+162 t-108\right) / 27,\right. \\
& \quad\left(637 t^{8}-378 t^{7}-1485 t^{6}+3186 t^{5}-1755 t^{4}-1782 t^{3}+3942 t^{2}-243 t-972\right) \\
&\times 4 t /(81 \sqrt{-3}))
\end{aligned}
$$

of heights $\frac{7}{6}$ and $\frac{21}{4}$ respectively, orthogonal with respect to the height pairing. Therefore, the Mordell-Weil rank is least 2. By Oda's calculations [1982], we deduce that the Mordell-Weil rank is exactly 2. The sections $P_{1}$ and $P_{2}$ and the trivial lattice span a lattice of discriminant $1000188=2^{2} \cdot 3^{6} \cdot 7^{3}$. Checking that it is saturated at 2, 3 and 7, we deduce that it must be the full Néron-Severi lattice. 


\begin{tabular}{|c|c|}
\hline$(g, h)$ & Sextic polynomial $f_{6}(x)$ defining the genus- 2 curve $y^{2}=f_{6}(x)$ \\
\hline$\left(\frac{11}{152},-\frac{16}{19}\right)$ & $-2332 x^{6}-902 x^{5}-5060 x^{4}-17111 x^{3}-5995 x^{2}-17545 x-27951$ \\
\hline & $-8788 x^{6}+34200 x^{5}-22425 x^{4}-11907 x^{3}-16230 x^{2}-35604 x+34024$ \\
\hline$\left(\frac{72}{49},-\frac{13}{7}\right)$ & $816 x^{6}-1944 x^{5}-13459 x^{4}+24712 x^{3}+37733 x^{2}+7596 x-2300$ \\
\hline$\left(\frac{75}{26},-\frac{19}{4}\right)$ & $\begin{array}{r}-106605 x^{6}-62661 x^{5}+467345 x^{4}+193313 x^{3}-691816 x^{2} \\
-149592 x+346546\end{array}$ \\
\hline$\left(\frac{12}{5},-10\right)$ & $\begin{array}{r}139968 x^{6}+471744 x^{5}-1301409 x^{4}-77363 x^{3}+671633 x^{2} \\
+236496 x+18756\end{array}$ \\
\hline$\left(-\frac{72}{31},-4\right)$ & $\begin{aligned} &-138482 x^{6}+1643417 x^{5}+2645029 x^{4}-1507309 x^{3}-2429567 x^{2} \\
&+ 1188320 x+36288\end{aligned}$ \\
\hline$\left(\frac{2}{135},-\frac{10}{11}\right)$ & $\begin{aligned} 2175200 x^{6}-2750760 x^{5}+2725545 x^{4}+7678368 x^{3}- & 5205621 x^{2} \\
& +3781674 x+9014158\end{aligned}$ \\
\hline-5$)$ & $\begin{array}{r}3829988 x^{6}+11621820 x^{5}-19617225 x^{4}-25097450 x^{3}+29201451 x^{2} \\
+14626080 x-14560512\end{array}$ \\
\hline$\left(\frac{18}{103},-\frac{8}{11}\right)$ & $\begin{array}{r}-4788022 x^{6}-21151494 x^{5}-18288935 x^{4}-20340320 x^{3}-61042325 x^{2} \\
+10128456 x-40124160\end{array}$ \\
\hline$\left(-\frac{39}{76},-\frac{29}{16}\right)$ & $\begin{aligned} 394632000 x^{6}-1964113200 x^{5}- & 1523778060 x^{4}+4757784967 x^{3} \\
+ & 148400811 x^{2}-3811137819 x+540964447\end{aligned}$ \\
\hline$\left(\frac{55}{117},-\frac{79}{144}\right)$ & $\begin{array}{r}-5511986931 x^{6}+20881501795 x^{5}+17115817125 x^{4}+19864594645 x^{3} \\
+1353729618 x^{2}-16117938900 x+5833685448\end{array}$ \\
\hline$\left(\frac{49}{135},-\frac{61}{75}\right)$ & $\begin{aligned}-26148549648 x^{6}- & 278797809744 x^{5}-748507062651 x^{4}+329438683288 x^{3} \\
+ & 1420002530997 x^{2}-1751808944796 x+1934174962804\end{aligned}$ \\
\hline$\left(-\frac{50}{117},-\frac{13}{9}\right)$ & $\begin{array}{l}-159682912000 x^{6}-1077016472800 x^{5}-2039981245815 x^{4} \\
-762577047304 x^{3}+6811301171385 x^{2}-4055008902300 x+449504680500\end{array}$ \\
\hline$\left(\frac{81}{91},-\frac{13}{7}\right)$ & $\begin{array}{r}134236157214 x^{6}+962817170858 x^{5}-12198892111873 x^{4}+23659009829816 x^{3} \\
+9649525790385 x^{2}-12776814846900 x-8264106337500\end{array}$ \\
\hline$\left(\frac{59}{153},-\frac{17}{27}\right)$ & $\begin{array}{r}-687158622928816 x^{6}+23483931596064 x^{5}-14038441316573 x^{4} \\
-893569395800 x^{3}-20141231607 x^{2}-200112822 x-748062\end{array}$ \\
\hline
\end{tabular}

Table 15. Some rational points $(g, h)$ of small height on the surface of Theorem 30 and the corresponding genus- 2 curves.

20.3. Examples. Table 15 lists some points of small height and their genus- 2 curves.

We could not find any curves of genus 0 which were not contained in the "bad locus" where the abelian surfaces have a strictly larger ring of endomorphisms.

\section{Discriminant 56}

21.1. Parametrization. We start with a $\mathrm{K} 3$ elliptic surface with $D_{6}, A_{8}$ and $A_{1}$ fibers, and a section $P$ of height $\frac{56}{72}=\frac{7}{9}=4-1-\frac{20}{9}$. The Weierstrass equation of this family is

$$
y^{2}=x^{3}+a x^{2}+2 b t^{2}(\lambda t-\mu) x+c t^{4}(\lambda t-\mu)^{2},
$$


with

$$
\begin{aligned}
& \lambda=\left(2 h-g^{2}+1\right), \quad \mu=-2 h, \\
& c=-\left(g^{2}-1\right)^{2}\left(4\left(g^{2}-1\right) t-(h+2)^{2}\right), \\
& b=\left(g^{2}-1\right)\left(2\left(g^{2}-1\right) t^{2}-\left(h^{2}+4 h-4 g^{2}+8\right) t-(h+2)^{2}\right), \\
& a=\left(g^{2}-1\right)^{2} t^{3}+(h+2)\left(h-2 g^{2}+4\right) t^{2}+2\left(h^{2}+4 h-2 g^{2}+6\right) t+(h+2)^{2} .
\end{aligned}
$$

To find an $E_{8} E_{7}$ fibration on such a $\mathrm{K} 3$ surface, we first identify the class of an $E_{7}$ fiber below.

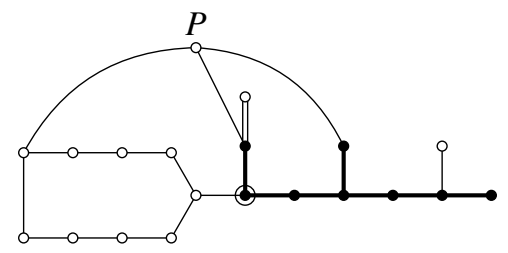

This converts it to an $A_{8} E_{7}$ fibration. Note that this fibration has a section, because the section $P$ intersects $F^{\prime}$ in 3, whereas (for instance) the non-identity component of the $A_{1}$ fiber intersects $F^{\prime}$ in 2, and these two numbers are coprime.

The new fibration has a section of height $\frac{56}{18}=\frac{28}{9}=4-\frac{8}{9}$, which intersects the identity component of the $E_{7}$ fiber and component 1 of the $A_{8}$ fiber. We then do a 3-neighbor step to get an $E_{8} E_{7}$ fibration.

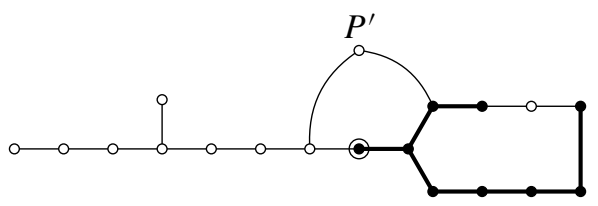

The new fiber $F^{\prime \prime}$ satisfies $P^{\prime} \cdot F^{\prime \prime}=2$, while the excluded component of the $A_{8}$ fiber intersects $F^{\prime \prime}$ in 3 . Since these are coprime, the fibration defined by $F^{\prime \prime}$ has a section.

Now we can read out the Igusa-Clebsch invariants, and describe the branch locus of $Y_{-}(56)$ as a double over of $\mathbb{P}_{g, h}^{2}$.

Theorem 31. A birational model over $\mathbb{Q}$ for the Hilbert modular surface $Y_{-}(56)$ as a double cover of $\mathbb{P}_{g, h}^{2}$ is given by the equation

$$
\begin{aligned}
z^{2}=\left(2 h-g^{2}+1\right)\left(2 h^{5}+27 g^{2} h^{4}-11 h^{4}+72 g^{2} h^{3}-24 h^{3}-40 g^{2} h^{2}\right. \\
\left.+104 h^{2}-160 g^{2} h+192 h+64 g^{4}-144 g^{2}+80\right) .
\end{aligned}
$$

It is a surface of general type. 
21.2. Analysis. The extra involution is $g \mapsto-g$. The branch locus has two components. Points on the simpler component $2 h-g^{2}+1=0$ (which is clearly a rational curve) correspond to elliptic K3 surfaces for which the $A_{1}$ and $D_{6}$ fibers merge and get promoted to a $D_{8}$ fiber. The other component corresponds to elliptic K3 surfaces with an extra $\mathrm{I}_{2}$ fiber. It is also of genus 0 , and a parametrization is given by

$$
(g, h)=\left(\frac{s\left(s^{4}+22 s^{2}-7\right)}{\left(3 s^{2}+1\right)^{2}},-\frac{\left(s^{2}-1\right)\left(s^{2}-5\right)}{2\left(3 s^{2}+1\right)}\right) .
$$

The Hilbert modular surface $Y_{-}(56)$ is of general type.

We now analyze the quotient of the Hilbert modular surface by the involution $(g, h, z) \mapsto(-g, h, z)$. Setting $f=g^{2}$, the right-hand side becomes a cubic in $f$. After some elementary Weierstrass transformations, we get the equation

$$
y^{2}=x^{3}-\left(27 h^{4}+72 h^{3}-40 h^{2}+96 h-16\right) x^{2}+512 h^{3}\left(7 h^{2}+20 h-4\right) x .
$$

This is an elliptic K3 surface, with reducible fibers of type $\mathrm{I}_{6}, \mathrm{I}_{6}, \mathrm{I}_{4}, \mathrm{I}_{3}$ at $h=$ $0, \infty,-2, \frac{2}{9}$ respectively, and $\mathrm{I}_{2}$ fibers at $h=(-10 \pm 8 \sqrt{2}) / 7$. The trivial lattice has rank 19 and discriminant 1728 . There is an obvious 2-torsion section $P_{0}=(0,0)$, and we find a non-torsion section

$$
P_{1}=\left(128 h, 128 h(h+2)^{2}\right)
$$

of height $\frac{2}{3}$. We checked that the group generated by $P_{0}$ and $P_{1}$ is saturated at 2 and 3. Therefore, this is a singular K3 surface, with Néron-Severi lattice of rank 20 and discriminant -288 .

Next, we analyze the twist of the elliptic K3 surface above, obtained by substituting $z=w g$ in the equation of the Hilbert modular surface, and then setting $f=g^{2}$ (it is the quotient of $Y_{-}(56)$ by the involution $(g, h, z) \mapsto(-g, h,-z)$ ). This twist is an honestly elliptic surface, with $\chi=3$. After some simple algebra, the Weierstrass equation can be written as

$$
\begin{aligned}
y^{2}=x^{3}+\left(58 h^{5}+149 h^{4}-56 h^{3}-\right. & \left.152 h^{2}-64 h+16\right) x^{2} \\
& +8 h^{3}(2 h+5)\left(h^{2}-4 h-4\right)^{2}\left(7 h^{2}+20 h-4\right) x .
\end{aligned}
$$

It has reducible fibers of type $\mathrm{I}_{0}^{*}, \mathrm{I}_{6}, \mathrm{I}_{4}, \mathrm{I}_{3}, \mathrm{I}_{2}, \mathrm{I}_{2}$ at $h=\infty, 0,-2, \frac{2}{9},-\frac{1}{2},-\frac{5}{2}$ respectively, $\mathrm{I}_{4}$ fibers at $h=(-10 \pm 8 \sqrt{2}) / 7$ and $\mathrm{I}_{2}$ fibers at $h=2 \pm 2 \sqrt{2}$. The trivial lattice has rank 26. In addition to the 2-torsion section $P_{0}=(0,0)$, we find the sections

$$
\begin{aligned}
& P_{1}=\left(-56 h^{3}\left(h^{2}-4 h-4\right), 16 \sqrt{14} h^{3}(2 h+1)(9 h-2)\left(h^{2}-4 h-4\right)\right), \\
& P_{2}=\left(-8 h^{3}\left(7 h^{2}+20 h-4\right), 64 \sqrt{-1} h^{3}(2 h+1)\left(7 h^{2}+20 h-4\right)\right), \\
& P_{3}=\left(4 h^{2}\left(7 h^{2}+20 h-4\right), 4 h^{2}(h+2)^{2}(2 h+1)\left(7 h^{2}+20 h-4\right)\right)
\end{aligned}
$$


of heights $\frac{5}{6}, 2$ and $\frac{7}{6}$ respectively, orthogonal with respect to the height pairing. On the other hand, counting points on the reductions modulo 11 and 29 shows that the Picard number is at most 29. Therefore, it is exactly 29. The lattice spanned by these sections and the trivial lattice has discriminant $35840=2^{10} \cdot 5 \cdot 7$. We checked that it is 2 -saturated, and therefore it must equal the entire Néron-Severi lattice.

21.3. Examples. Table 16 lists some points of small height and their genus- 2 curves.

Next, we analyze curves of low genus on the Hilbert modular surface. The specialization $h=\frac{2}{9}$ gives a rational curve, parametrized by $g=\left(m^{2}-4 m-9\right) /\left(m^{2}+9\right)$. The Brauer obstruction vanishes identically for rational points on this curve, giving a 1parameter family of genus- 2 curves whose Jacobians have real multiplication by $\mathcal{O}_{56}$.

The specializations $h=-\frac{1}{2}$ and $h=-\frac{5}{2}$ give genus- 1 curves with rational points, both of whose Jacobians have rank 1. The Brauer obstruction does not vanish identically on either of these loci.

We also obtain some genus- 1 curves by pulling back some sections from the quotient K3 surface. For instance, the section $P_{0}+P_{1}$ gives the genus- 1 curve $g^{2}=-\left(7 h^{4}+20 h^{3}-4 h^{2}-32 h-16\right) / 16$ which has rational points (such as

\begin{tabular}{l|c}
$(g, h)$ & Sextic polynomial $f_{6}(x)$ defining the genus-2 curve $y^{2}=f_{6}(x)$ \\
\hline$\left(-\frac{79}{61}, \frac{28}{61}\right)$ & $-2000 x^{6}+2040 x^{5}-565 x^{4}+628 x^{3}-349 x^{2}-36 x-68$ \\
$\left(\frac{23}{19}, \frac{24}{95}\right)$ & $480 x^{6}+1200 x^{5}+2657 x^{4}+1264 x^{3}+497 x^{2}-2220 x+660$ \\
$\left(\frac{2}{13},-\frac{6}{13}\right)$ & $-600 x^{6}-360 x^{5}+2660 x^{4}+256 x^{3}-2698 x^{2}-222 x+639$ \\
$\left(-\frac{2}{13},-\frac{6}{13}\right)$ & $1096 x^{6}-24 x^{5}-3388 x^{4}+608 x^{3}+2750 x^{2}-930 x-225$ \\
$\left(\frac{79}{61}, \frac{28}{61}\right)$ & $1350 x^{6}+270 x^{5}+3375 x^{4}-3944 x^{3}+1669 x^{2}-5328 x+3392$ \\
$\left(-\frac{3}{7},-\frac{8}{7}\right)$ & $-1340 x^{6}+5900 x^{5}-2227 x^{4}+5096 x^{3}+2707 x^{2}+10 x+1950$ \\
$\left(\frac{3}{7},-\frac{8}{7}\right)$ & $1440 x^{6}-4720 x^{5}-13227 x^{4}+20 x^{3}+7389 x^{2}-1080 x-432$ \\
$\left(-\frac{1}{7}, \frac{24}{7}\right)$ & $-12600 x^{6}-3192 x^{5}-16975 x^{4}-4442 x^{3}+5717 x^{2}-516 x+4$ \\
$\left(\frac{1}{91},-\frac{40}{91}\right)$ & $3740 x^{6}-6420 x^{5}-11789 x^{4}+18160 x^{3}+7315 x^{2}-13356 x+2268$ \\
$\left(-\frac{11}{7}, \frac{9}{7}\right)$ & $35220 x^{6}+10548 x^{5}+43345 x^{4}-10038 x^{3}+3313 x^{2}-228 x+52$ \\
$\left(-\frac{23}{19}, \frac{24}{95}\right)$ & $47824 x^{6}+45048 x^{5}+13973 x^{4}-11016 x^{3}+9341 x^{2}-2040 x+400$ \\
$\left(\frac{2}{3}, \frac{2}{9}\right)$ & $-6883 x^{6}+10038 x^{5}+62514 x^{4}+31744 x^{3}-21780 x^{2}+3720 x-200$ \\
$\left(\frac{37}{31}, \frac{9}{31}\right)$ & $-1548 x^{6}-7732 x^{5}-33547 x^{4}-51202 x^{3}-71163 x^{2}+65988 x-11772$ \\
$\left(-\frac{4}{17},-\frac{3}{8}\right)$ & $-316 x^{6}+4764 x^{5}+21121 x^{4}-11666 x^{3}-75071 x^{2}+20364 x+49716$ \\
$\left(\frac{11}{7}, \frac{9}{7}\right)$ & $25092 x^{6}+70500 x^{5}+71881 x^{4}-29834 x^{3}-80543 x^{2}-25908 x+38700$ \\
$\left(\frac{55}{13}, \frac{28}{3}\right)$ & $-94 x^{6}-114 x^{5}-2497 x^{4}-660 x^{3}-29263 x^{2}-10920 x-170352$ \\
\hline
\end{tabular}

Table 16. Some rational points $(g, h)$ of small height on the surface of Theorem 31 and the corresponding genus- 2 curves. 
$(h, g)=(1, \pm 1))$, with a Jacobian of conductor $2^{4} 211$ and Mordell-Weil group $\cong \mathbb{Z}^{2}$. The section $2 P_{1}$ gives the genus- 1 curve $g^{2}=-\left(49 h^{4}-112 h^{3}+64 h^{2}-32 h-16\right) / 16$ which also has rational points $(h, g)=(1, \pm 1)$, with Jacobian of conductor $2^{6} 7 \cdot 23$ and Mordell-Weil group $\cong(\mathbb{Z} / 2 \mathbb{Z}) \oplus \mathbb{Z}$. The Brauer obstruction does not vanish identically on either of these loci.

\section{Discriminant 57}

22.1. Parametrization. We start with an elliptic $\mathrm{K} 3$ surface with fibers of type $E_{6}$, $A_{7}$ and $A_{2}$ at $t=\infty, 0,1$ respectively, and a section of height $\frac{57}{72}=\frac{19}{24}=4-\frac{4}{3}-\frac{3 \cdot 5}{8}$.

The Weierstrass equation for this family is

$$
y^{2}=x^{3}+a x^{2}+2 b t^{2}(t-1) x+c t^{4}(t-1)^{2},
$$

with

$$
\begin{aligned}
a= & t(t-1)\left(\left(g^{2}-\frac{1}{4}\right)\left(h^{2}-3\right)+2 g h\right)+\left(\left(g^{2}+\frac{1}{4}\right) h-g\right)^{2} t^{2}-t+1, \\
b= & -\left(g^{2}-\frac{1}{4}\right)^{2}\left(h^{2}-1\right) \\
& \quad\left((1-t)-\left(\left(g^{2}-\frac{1}{4}\right)\left(h^{2}-2\right)+2 g h\right) t(1-t)-\left(g-\frac{1}{2} h\right)\left(\left(g^{2}+\frac{1}{4}\right) h-g\right) t^{2}\right), \\
c= & \left(g^{2}-\frac{1}{4}\right)^{4}\left(h^{2}-1\right)^{2}\left((1-t)+\left(g-\frac{1}{2} h\right)^{2} t^{2}+t(1-t)\left(\left(g-\frac{1}{2} h\right)^{2}-\left(g h+\frac{1}{2}\right)^{2}\right)\right) .
\end{aligned}
$$

We identify an $E_{8}$ fiber below, and the resulting 3-neighbor step takes us to an elliptic fibration with $E_{8}$ and $A_{7}$ fibers.

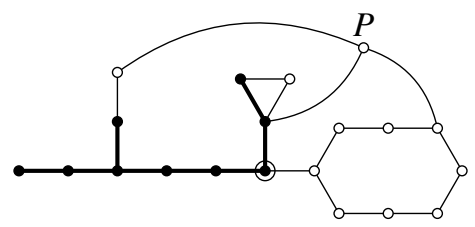

Since $P \cdot F^{\prime}=2$ for the new fiber $F^{\prime}$, while the intersection number of the remaining component of the $E_{6}$ fiber with $F^{\prime}$ is 3, we deduce that the new fibration has a section.

The new fibration has a section of height $\frac{57}{8}=4+2 \cdot 2-\frac{1 \cdot 7}{8}$. Now we can identify an $E_{7}$ fiber $F^{\prime \prime}$ and move to the $E_{8} E_{7}$ fibration by a 2-neighbor step. Note that since $Q \cdot F^{\prime \prime}=7$, while the remaining component of the $A_{7}$ fiber intersects $F^{\prime \prime}$ in 2 , the new fibration will have a section.

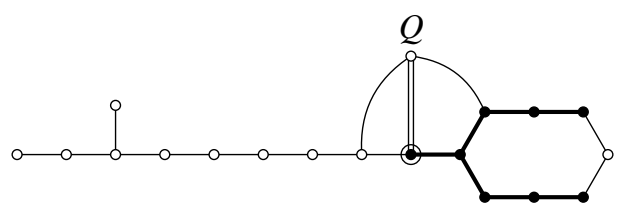


Now we can read out the Igusa-Clebsch invariants and compute the branch locus, which is the subfamily with an extra $I_{2}$ fiber.

Theorem 32. A birational model over $\mathbb{Q}$ for the Hilbert modular surface $Y_{-}(57)$ as a double cover of $\mathbb{P}_{g, h}^{2}$ is given by the equation

$$
\begin{aligned}
& z^{2}=\left(256 g^{6}-176 g^{4}+40 g^{2}-3\right) h^{4}+\left(2176 g^{5}-960 g^{3}+104 g\right) h^{3} \\
& +\left(4320 g^{4}-688 g^{2}-34\right) h^{2}+\left(-3456 g^{5}+576 g^{3}+328 g\right) h-2160 g^{4}-648 g^{2}+361 .
\end{aligned}
$$

It is an honestly elliptic surface, with arithmetic genus 2 and Picard number 29.

22.2. Analysis. The extra involution is $(g, h) \mapsto(-g,-h)$. The branch locus has genus 2 ; the transformation

$$
(g, h)=\left(\frac{y+x^{2}+1}{4 x^{3}}, \frac{x\left(4 y-x^{4}-14 x^{2}-1\right)}{\left(x^{2}-1\right)^{2}}\right)
$$

converts it to Weierstrass form

$$
y^{2}=3 x^{6}+11 x^{4}+x^{2}+1 .
$$

It is isomorphic to the quotient of $X_{0}(57)$ by the Atkin-Lehner involution $w_{57}$.

The Hilbert modular surface $Y_{-}(57)$ is an honestly elliptic surface, with a genus-1 fibration over $\mathbb{P}_{g}^{1}$, and in fact, setting $h=1$ gives a section. Therefore, we may use the Jacobian form, which has the Weierstrass equation

$$
\begin{aligned}
& y^{2}=x^{3}+4\left(12 g^{2}-1\right)\left(28 g^{2}-5\right) x^{2} \\
& -4(2 g-1)^{3}(2 g+1)^{3}\left(12 g^{2}-5\right)\left(108 g^{2}-19\right) x+(2 g-1)^{6}(2 g+1)^{6}\left(108 g^{2}-19\right)^{2} .
\end{aligned}
$$

It has reducible fibers of type $\mathrm{I}_{7}$ at $g= \pm \frac{1}{2}$, IV at $g=\infty, \mathrm{I}_{2}$ at $g= \pm \frac{\sqrt{57}}{18}$, and $\mathrm{I}_{3}$ at the four roots of $432 g^{4}+216 g^{2}-49$ (which generate a dihedral extension containing $\mathbb{Q}(\sqrt{57})$ ). The trivial lattice contributes 26 to the rank of the Néron-Severi lattice, leaving room for Mordell-Weil rank at most 4 . We find the sections

$$
\begin{aligned}
& P_{1}=\left(0,(2 g-1)^{3}(2 g+1)^{3}\left(108 g^{2}-19\right)\right), \\
& P_{2}=\left((2 g-1)^{2}(2 g+1)^{2}(6 g-1)(6 g+1),\right. \\
& \left.\quad 2 g(2 g-1)^{2}(2 g+1)^{2}\left(432 g^{4}+216 g^{2}-49\right)\right), \\
& P_{3}=\left((\mu+3)(18 g-\mu)(2 g-1)(2 g+1)^{3} / 3,\right. \\
& \left.\quad\left(36 g^{2}+9-2 \mu\right)(2 g+6+\mu)(18 g-\mu)(2 g-1)(2 g+1)^{3} / 3\right)
\end{aligned}
$$

(where $\mu=\sqrt{57}$ ), with nondegenerate height pairing matrix

$$
\frac{1}{42}\left(\begin{array}{rrr}
38 & 0 & -19 \\
0 & 20 & -10 \\
-19 & -10 & 39
\end{array}\right) \text {. }
$$


Therefore, the Picard number is at least 29. In fact, counting points modulo 7 and 11 shows that the Picard number must be exactly 29 . Alternatively, analysis of the quotients below gives another proof. The sections above together with the trivial lattice span a lattice of discriminant $11970=2 \cdot 3^{2} \cdot 5 \cdot 7 \cdot 19$. We checked that it 3 -saturated, and thus it is all of the Néron-Severi lattice. Therefore, these sections generate the Mordell-Weil group.

Next, we analyze the quotient by the involution $(g, h, z) \mapsto(-g,-h, z)$. This turns out to have a genus-1 fibration with section over the $t$-line, where $t=g^{2}$. The Weierstrass equation may be written (after a linear change of the base parameter and a Weierstrass transformation)

$$
\begin{aligned}
y^{2}=x^{3}+4(t+1)(3 t+2) & (7 t+2) x^{2} \\
& -4 t^{3}(t+1)^{2}(3 t-2)(27 t+8) x+t^{6}(t+1)^{3}(27 t+8)^{2} .
\end{aligned}
$$

This is an elliptic K3 surface with fibers of type $\mathrm{I}_{7}$ at $t=0, \mathrm{I}_{0}^{*}$ at $t=-1, \mathrm{I}_{2}$ at $t=-\frac{8}{27}$, II at $t=\infty$, and $\mathrm{I}_{3}$ at $t=-2 \pm \frac{2 \sqrt{57}}{9}$. Thus the trivial lattice has rank 17, leaving room for at most three independent sections.

We find the independent sections

$$
\begin{aligned}
& P_{1}=\left(t^{2}(t+1)(9 t+8), t^{2}(t+1)^{2}\left(27 t^{2}+108 t+32\right)\right), \\
& P_{2}=\left((t+1)(27 t+8)\left(3 t^{2}-64 t-64\right) / 57,\right. \\
& \left.\qquad(t+1)^{2}(t+40)(27 t+8)\left(27 t^{2}+108 t+32\right) / 57^{3 / 2}\right)
\end{aligned}
$$

of heights $\frac{5}{21}$ and $\frac{7}{6}$ respectively, and orthogonal with respect to the height pairing. Therefore the Picard number is at least 19. Counting points modulo 11 and 13 shows that the Picard number cannot be 20 . These sections together with the trivial lattice generate a lattice of discriminant 140 . We check that it is 2-saturated and must therefore be the full Néron-Severi lattice.

Replacing $g^{2}$ by $t$ in the Weierstrass equation for $Y_{-}(57)$ gives a quadratic twist of the above quotient $\mathrm{K} 3$ surface, given by the Weierstrass equation

$$
\begin{aligned}
y^{2}=x^{3}+4(12 t-1) & (28 t-5) x^{2} \\
& -4(4 t-1)^{3}(12 t-5)(108 t-19) x+(4 t-1)^{6}(108 t-19)^{2} .
\end{aligned}
$$

This is an elliptic K3 surface with fibers of type $\mathrm{I}_{7}$ at $t=\frac{1}{4}, \mathrm{I}_{2}$ at $t=\frac{19}{108}, \mathrm{I}_{3}$ at $t=-\frac{1}{4} \pm \frac{\sqrt{57}}{18}$ and $\mathrm{IV}^{*}$ at $t=\infty$. The section $P=\left(0,(4 t-1)^{3}(108 t-19)\right)$ has height $\frac{19}{42}$. Therefore, this K3 surface is singular. Together with the trivial lattice, the section $P$ spans a lattice of discriminant $171=3^{2} \cdot 19$. Since there is no 3 -torsion section, and we cannot have a section of height $19 /\left(3^{2} \cdot 42\right)$ due to the configuration of fibers, this must be the full Néron-Severi lattice.

Therefore, the Mordell-Weil rank of the original surface must be $2+1=3$. 


\begin{tabular}{l|c}
$(g, h)$ & Sextic polynomial $f_{6}(x)$ defining the genus-2 curve $y^{2}=f_{6}(x)$ \\
\hline$\left(-\frac{1}{10},-\frac{5}{3}\right)$ & $-2 x^{6}+15 x^{5}+131 x^{4}+240 x^{3}-61 x^{2}-8$ \\
$\left(\frac{7}{10},-\frac{29}{3}\right)$ & $81 x^{6}+54 x^{5}-286 x^{4}-186 x^{3}+323 x^{2}+120 x-130$ \\
$\left(\frac{7}{18}, 9\right)$ & $-108 x^{6}+324 x^{5}-243 x^{4}+186 x^{3}-279 x^{2}-80$ \\
$\left(-\frac{7}{6}, \frac{21}{5}\right)$ & $-100 x^{6}-390 x^{5}-204 x^{4}+74 x^{3}-69 x^{2}-12 x+8$ \\
$\left(-\frac{1}{18},-3\right)$ & $-220 x^{6}+420 x^{5}-111 x^{4}+238 x^{3}+381 x^{2}+168 x+24$ \\
$\left(-\frac{1}{4},-\frac{16}{3}\right)$ & $95 x^{6}-114 x^{5}+325 x^{4}+35 x^{3}+10 x^{2}-429 x-234$ \\
$\left(\frac{5}{14}, \frac{35}{3}\right)$ & $120 x^{6}-192 x^{5}+122 x^{4}+286 x^{3}-448 x^{2}+357 x-63$ \\
$\left(\frac{19}{18},-\frac{3}{5}\right)$ & $58 x^{6}+39 x^{5}-129 x^{4}+132 x^{3}+519 x^{2}+240 x-40$ \\
$\left(-\frac{7}{10}, \frac{29}{3}\right)$ & $-390 x^{6}+451 x^{5}-230 x^{4}-593 x^{3}+682 x^{2}+220 x-200$ \\
$\left(\frac{4}{9}, 18\right)$ & $-540 x^{6}+729 x^{5}-135 x^{4}+255 x^{3}-225 x^{2}-36 x-52$ \\
$\left(-\frac{5}{2},-\frac{10}{3}\right)$ & $-60 x^{6}-156 x^{5}+137 x^{4}+310 x^{3}-351 x^{2}+108 x+756$ \\
$\left(-\frac{17}{10},-\frac{47}{33}\right)$ & $60 x^{5}+839 x^{4}+278 x^{3}-652 x^{2}-36 x-489$ \\
$\left(\frac{1}{18}, 3\right)$ & $-60 x^{6}+60 x^{5}-3 x^{4}+184 x^{3}+669 x^{2}+132 x+868$ \\
$\left(\frac{5}{2}, \frac{10}{3}\right)$ & $-819 x^{5}-1042 x^{4}+61 x^{3}+248 x^{2}-48 x$ \\
$\left(-\frac{1}{18}, \frac{9}{5}\right)$ & $-40 x^{6}-72 x^{5}-45 x^{4}-534 x^{3}-297 x^{2}-324 x-1188$ \\
$\left(-\frac{5}{2}, \frac{5}{3}\right)$ & $-36 x^{6}+84 x^{5}+491 x^{4}-750 x^{3}-337 x^{2}-912 x-1200$ \\
\hline
\end{tabular}

Table 17. Some rational points $(g, h)$ of small height on the surface of Theorem 32 and the corresponding genus- 2 curves.

22.3. Examples. Table 17 lists some points of small height and their genus-2 curves.

The specialization $h=0$ gives a genus- 1 curve with rational points, whose Jacobian has rank 1 . Of course, there is a large supply of genus-1 curves, simply by specializing $g$, since we have an elliptic surface.

The sections $P_{1}$ and $P_{2}$ give rational curves $h=-16 g /\left(4 g^{2}-1\right)$ and $h=$ $\left(72 g^{3}-36 g^{2}+78 g-7\right) /\left(\left(4 g^{2}-1\right)(6 g-7)\right)$ respectively. The Brauer obstruction vanishes on these curves.

\section{Discriminant 60}

23.1. Parametrization. We start with a $\mathrm{K} 3$ elliptic surface with $E_{6}, D_{6}, A_{4}$ fibers at $\infty, 0,1$ respectively. The Weierstrass equation for this family is

$$
y^{2}=x^{3}+a t x^{2}+2 b t^{3}(t-1) x+c t^{5}(t-1)^{2},
$$


with

$$
\begin{aligned}
& a=\left(h^{2}-g^{2}-1\right)^{2}-4\left(2 h^{2}-3 g^{2}+6\right)(t-1), \\
& b=4\left(-4\left(h^{2}-g^{2}-1\right)^{2}+16\left(h^{2}-2 g^{2}+1\right)(t-1)\right) \\
& c=256\left(\left(h^{2}-g^{2}-1\right)^{2}+4 g^{2}(t-1)\right) .
\end{aligned}
$$

We identify an $E_{8}$ fiber below, and move to this elliptic fibration by a 3-neighbor step.

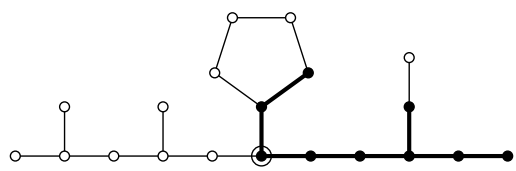

The new elliptic fibration has fibers of type $E_{8}, D_{6}$ and $A_{1}$, and a section $P$ of height $\frac{60}{8}=\frac{15}{2}=4+2 \cdot 2-\frac{1}{2}$. We now identify an $E_{7}$ fiber $F^{\prime}$ and perform a 2-neighbor step to go to the new fibration. Note that it has a section, since $P \cdot F^{\prime}=7$, while the remaining component of the $D_{6}$ fiber has intersection number 2 with $F^{\prime}$.

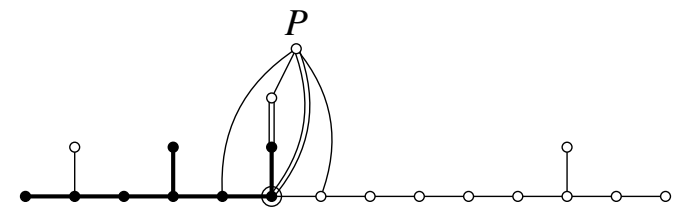

From the resulting $E_{8} E_{7}$ fibration, we can read out the map to $\mathcal{A}_{2}$.

Theorem 33. A birational model over $\mathbb{Q}$ for the Hilbert modular surface $Y_{-}(60)$ as a double cover of $\mathbb{P}_{g, h}^{2}$ is given by the equation

$$
\begin{aligned}
z^{2} & =-\left(h^{2}-2 h-g^{2}+5\right)\left(h^{2}+2 h-g^{2}+5\right) \\
& \times\left(8 h^{6}-25 g^{2} h^{4}+24 h^{4}+26 g^{4} h^{2}-86 g^{2} h^{2}+24 h^{2}-9 g^{6}+66 g^{4}+47 g^{2}+8\right) .
\end{aligned}
$$

It is a surface of general type.

23.2. Analysis. The surface $Y_{-}(60)$ has two extra commuting involutions, $\iota_{1}$ : $(g, h) \mapsto(-g, h)$ and $\iota_{2}:(g, h) \mapsto(g,-h)$. The two simpler components

$$
h^{2} \pm 2 h-g^{2}+5=0
$$

of the branch locus correspond to the subfamily of elliptic K3 surfaces where the $D_{6}$ fiber gets promoted to an $E_{7}$ fiber, while the more complicated component corresponds to an extra $\mathrm{I}_{2}$ fiber. The simpler components are easily seen to be rational curves, as they define conics in the $(g, h)$-plane, with rational points. The last component is also a rational curve; a parametrization is given by

$$
(g, h)=\left(\frac{\left(t^{2}+1\right)^{3}}{t\left(t^{2}-1\right)\left(t^{2}-2 t-1\right)}, \frac{\left(t^{2}+2 t-1\right)\left(t^{4}-t^{3}+2 t^{2}+t+1\right)}{t\left(t^{2}-1\right)\left(t^{2}-2 t-1\right)}\right) .
$$


This Hilbert modular surface is of general type.

We now analyze some of the quotients of this surface by the involutions. The quotient under both involutions is given by

$$
\begin{aligned}
z^{2}=-\left(h^{2}-2 g h+6 h+g^{2}-10 g+25\right) & \\
& \left(8 h^{3}-25 g h^{2}+24 h^{2}+26 g^{2} h-86 g h+24 h-9 g^{3}+66 g^{2}+47 g+8\right),
\end{aligned}
$$

and this is actually a rational surface; the transformation

$$
(g, h)=\left(h^{\prime}+g^{\prime 2}+2 g^{\prime}+5, h^{\prime}+g^{\prime 2}\right)
$$

converts the above equation into a conic bundle over $\mathbb{P}_{h^{\prime}}^{1}$ with a section.

The quotient by the involution $\iota_{1}$ turns out to be an elliptic K3 surface, with Weierstrass equation given by

$$
\begin{aligned}
y^{2}=x^{3}+2 t^{2}\left(215 t^{2}+356 t+140\right) x^{2}-t^{3}(t & +2)^{3}\left(5 t^{2}+874 t+864\right) x / 3 \\
& -8 t^{4}(t+2)^{6}\left(163 t^{2}-54 t-216\right) / 27 .
\end{aligned}
$$

It has reducible fibers of type $E_{6}$ at $t=0, \mathrm{I}_{6}$ at $t=-2, \mathrm{I}_{2}$ at $t=-1$ and $t=-\frac{2}{9}$, and $\mathrm{I}_{3}$ at $t=(-7 \pm 5 \sqrt{5}) / 19$. The trivial lattice therefore has rank 19. We find a 3 -torsion section with $x$-coordinate $11 t^{2}(t+2)^{2} / 3$ and a non-torsion section with $x=-t^{2}(t+2)^{2} / 3$. Therefore the K3 surface is singular. These sections, together with the trivial lattice, generate a lattice of rank 20 and discriminant 60 . It must be the full Néron-Severi lattice, since otherwise there would have to be a 6-torsion section or section of height $\frac{5}{24}$, neither of which is possible with our configuration of reducible fibers.

The quotient by the involution $\iota_{2}$ is also an elliptic K3 surface, with Weierstrass equation

$$
y^{2}=x^{3}-\left(11 t^{4}-20 t^{2}+8\right) x^{2}+16(t-1)^{3}(t+1)^{3}\left(4 t^{2}-5\right) x .
$$

This has bad fibers of type $\mathrm{I}_{6}$ at $t= \pm 1, \mathrm{I}_{2}$ at $t= \pm \frac{\sqrt{5}}{2}$, and $\mathrm{I}_{3}$ at $t= \pm \frac{2}{\sqrt{3}}$. Therefore the trivial lattice has rank 18 , leaving room for at most two independent sections. We find the following sections, of which the first is 6-torsion.

$$
\begin{aligned}
& P_{0}=\left(2\left(t^{2}-1\right)\left(4 t^{2}-5\right), 4\left(t^{2}-1\right)\left(3 t^{2}-4\right)\left(4 t^{2}-5\right)\right), \\
& P_{1}=\left((11-3 \mu) t^{2}\left(t^{2}-1\right) / 2,(3+5 \mu) t\left(t^{2}-1\right) / 12\left(18 t^{2}-15+\mu\right)\right), \\
& P_{2}=\left((11+3 \mu) t^{2}\left(t^{2}-1\right) / 2,(3-5 \mu) t\left(t^{2}-1\right) / 12\left(18 t^{2}-15-\mu\right)\right),
\end{aligned}
$$

where $\mu=\sqrt{-15}$. The height pairing matrix of $P_{1}$ and $P_{2}$ is

$$
\frac{1}{3}\left(\begin{array}{rr}
7 & -2 \\
-2 & 7
\end{array}\right)
$$




\begin{tabular}{l|c}
$(g, h)$ & Sextic polynomial $f_{6}(x)$ defining the genus-2 curve $y^{2}=f_{6}(x)$ \\
\hline$\left(\frac{17}{6},-\frac{13}{6}\right)$ & $468 x^{6}+1332 x^{5}+1345 x^{4}-20 x^{3}+1051 x^{2}-150 x+186$ \\
$\left(-\frac{17}{6}, \frac{13}{6}\right)$ & $-12 x^{6}+132 x^{5}+371 x^{4}+1506 x^{3}+1391 x^{2}-528 x-1872$ \\
$\left(\frac{17}{6}, \frac{13}{6}\right)$ & $-942 x^{6}+3150 x^{5}-869 x^{4}-4220 x^{3}+745 x^{2}+2244 x+468$ \\
$\left(-\frac{17}{6},-\frac{7}{6}\right)$ & $48 x^{6}-360 x^{5}+1907 x^{4}-4000 x^{3}+5195 x^{2}+828 x+2556$ \\
$\left(-\frac{17}{6},-\frac{13}{6}\right)$ & $-4500 x^{6}-9300 x^{5}-5365 x^{4}+4106 x^{3}+3335 x^{2}-2112 x-2648$ \\
$\left(-\frac{17}{6}, \frac{7}{6}\right)$ & $-4116 x^{6}-6468 x^{5}+8617 x^{4}+11086 x^{3}-12239 x^{2}-3708 x+4212$ \\
$\left(\frac{17}{6}, \frac{7}{6}\right)$ & $72 x^{6}-2136 x^{5}+15869 x^{4}-258 x^{3}-1759 x^{2}+108 x-4$ \\
$\left(\frac{17}{6},-\frac{7}{6}\right)$ & $12 x^{6}-36 x^{5}+929 x^{4}-1458 x^{3}+16361 x^{2}-4452 x+1476$ \\
$\left(\frac{51}{5},-\frac{54}{5}\right)$ & $9248 x^{6}-2312 x^{5}+12427 x^{4}-29852 x^{3}-21811 x^{2}+26690 x+21270$ \\
$\left(\frac{57}{10}, \frac{43}{10}\right)$ & $2272 x^{6}+35064 x^{5}+12877 x^{4}-24234 x^{3}-37079 x^{2}+29700 x-3500$ \\
$\left(-\frac{51}{5},-\frac{54}{5}\right)$ & $-6368 x^{6}-20760 x^{5}+11991 x^{4}+29560 x^{3}-61443 x^{2}+39870 x-12150$ \\
$\left(-\frac{46}{15},-\frac{49}{15}\right)$ & $-575 x^{6}-3075 x^{5}-12269 x^{4}-16401 x^{3}-56024 x^{2}-21792 x-73242$ \\
$\left(-\frac{61}{10}, \frac{49}{10}\right)$ & $-36450 x^{6}-10530 x^{5}+6327 x^{4}+78760 x^{3}-29879 x^{2}-17700 x+2612$ \\
$\left(\frac{61}{10}, \frac{49}{10}\right)$ & $8612 x^{6}-4020 x^{5}-52381 x^{4}-4290 x^{3}+91787 x^{2}+47220 x-11540$ \\
$\left(\frac{61}{10},-\frac{49}{10}\right)$ & $-17092 x^{6}+13812 x^{5}-101885 x^{4}+63210 x^{3}-89229 x^{2}+69580 x-15092$ \\
$\left(-\frac{51}{5}, \frac{54}{5}\right)$ & $-100572 x^{6}-102884 x^{5}-147679 x^{4}-25432 x^{3}+27727 x^{2}+35870 x-11890$ \\
\hline
\end{tabular}

Table 18. Some rational points $(g, h)$ of small height on the surface of Theorem 33 and the corresponding genus- 2 curves.

Therefore the Picard number of this K3 surface is 20 . The discriminant of the sublattice of $\mathrm{NS}(X)$ generated by the trivial lattice and these sections is 180 . We checked that this lattice is 2- and 3-saturated, which proves that it is the entire Picard group.

23.3. Examples. Table 18 lists some points of small height and their genus-2 curves.

We now describe some curves of genus 1 , possessing infinitely many rational points, on the Hilbert modular surface. These were obtained by pulling back rational curves on the quotients by $\iota_{1}$ and $\iota_{2}$ obtained as sections of the elliptic fibrations. In each case we give the curve as a double cover of $\mathbb{P}^{1}$, exhibit a coordinate of a point on $\mathbb{P}^{1}$ that lifts to a rational point, and give the conductor and Mordell-Weil group.

- $v=\infty$, conductor $2^{4} 3^{2} 11 \cdot 97$, Mordell-Weil group $(\mathbb{Z} / 2 \mathbb{Z}) \oplus \mathbb{Z}$, equation

$$
g^{2}=\frac{v^{4}+132 v^{3}+11784 v^{2}+566280 v+20175732}{36(v+9)^{2}}, \quad h=\frac{v^{2}+72 v+4560}{6(v+9)} .
$$


- $v=1$, conductor $2 \cdot 3 \cdot 7 \cdot 17 \cdot 19$, Mordell-Weil group $(\mathbb{Z} / 2 \mathbb{Z})^{2} \oplus \mathbb{Z}$, equation

$$
g^{2}=\frac{4913 v^{4}+1990 v^{2}+153}{36\left(v^{2}-1\right)^{2}}, \quad h=-\frac{25 v^{2}+3}{2\left(v^{2}-1\right)} .
$$

- $t=-1$, conductor $5^{2} 11 \cdot 17 \cdot 47$, Mordell-Weil group $\mathbb{Z}^{2}$, equation

$$
g^{2}=\frac{14049 t^{4}-57248 t^{3}+87462 t^{2}-59840 t+15657}{(t+1)^{2}(9 t-11)^{2}}, \quad h=-\frac{2(t-1)(54 t-67)}{(t+1)(9 t-11)} .
$$

- $v=0$, conductor $238=2 \cdot 7 \cdot 17$, Mordell-Weil group $(\mathbb{Z} / 2 \mathbb{Z}) \oplus \mathbb{Z}$, equation

$$
h^{2}=\frac{833 v^{4}+190 v^{2}+1}{4\left(v^{2}-1\right)^{2}}, \quad g=\frac{27 v^{2}+5}{2\left(v^{2}-1\right)} .
$$

We can obtain a few more such curves from these, by applying the involution $\iota_{2}$ to the first three curves, and the involution $\iota_{1}$ to the last. If two genus- 2 curves are parametrized by points related by such an involution, then the curves' Jacobians are isogenous.

\section{Discriminant 61}

24.1. Parametrization. Start with an elliptic surface with $D_{7}, A_{6}$ and $A_{2}$ fibers and a section of height $\frac{61}{84}=4-\frac{2}{3}-\frac{6}{7}-\frac{7}{4}$.

The Weierstrass equation for this family can be written as

$$
y^{2}=x^{3}+a x^{2}+2 b t(1-1) x+c t^{2}(t-1)^{2},
$$

where

$$
\begin{gathered}
\begin{array}{c}
a=4 h^{3}(h-g)^{3} t^{3}+(h-g)^{2}\left(g^{2} h^{2}-4 g h^{2}-8 h^{2}-2 g^{2} h+g^{2}+12 g+12\right) t^{2} \\
-
\end{array} \\
\begin{array}{r}
b=-4(g+1)(h-g)\left(g^{2} h+4 h-g^{2}-6 g\right) t+g^{2}(g+1)^{2}, \\
+(h-g)^{2}( \\
(h-g)^{2}\left(2 g h^{2}+4 h^{2}+g^{2} h-g^{2}-6 g-6\right) t^{2} \\
\left.+(g+1)(h-g)\left(g^{2} h+2 h-2 g^{2}-6 g\right) t-g^{2}(g+1)^{2}\right),
\end{array} \\
c=16(g+1)^{2}(h-g)^{4}((g+2)(h-g) t+g(g+1))^{2} .
\end{gathered}
$$

We first perform a 2-neighbor step to move to an elliptic fibration with $E_{7}$ and $A_{7}$ fibers, by locating an $E_{7}$ fiber, as follows.

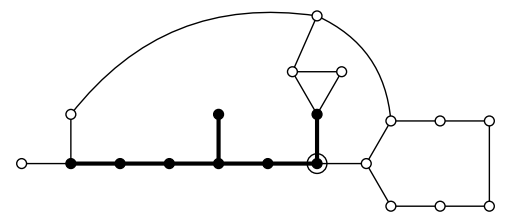


This elliptic fibration has Mordell-Weil rank 2, and we can in fact write down two generators of the Mordell-Weil group, which intersect the components of reducible fibers as shown below. Next, we identify the class of an $E_{8}$ fiber, and use it to perform 2-neighbor step to an elliptic fibration with $E_{8}, A_{5}$ and $A_{1}$ fibers, and Mordell-Weil rank 2.

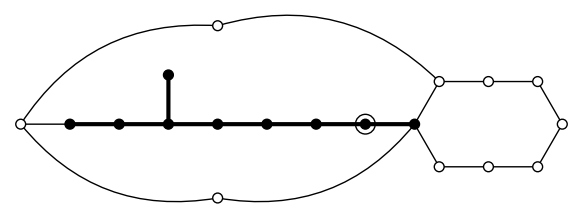

We show one of the generators $P$ of the Mordell-Weil lattice, which has height $4-2 \cdot \frac{4}{6}-\frac{1}{2}$. Next, we go to a fibration with $E_{8}$ and $E_{6}$ fibers using the fiber class $F^{\prime}$ of $E_{6}$ below. Since $P \cdot F^{\prime}=1$, the new elliptic fibration has a section.

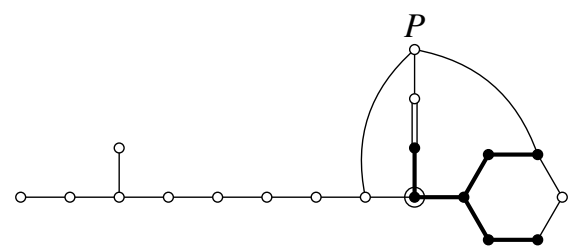

We can find a section $P^{\prime}$ of this elliptic fibration with $E_{8}$ and $E_{6}$ fibers, of height $\frac{8}{3}=4-\frac{4}{3}$. We use it to go to a fibration with $E_{8}$ and $E_{7}$ fibers as shown.

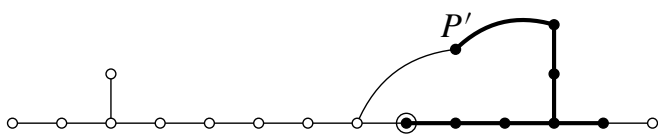

From the resulting $E_{8} E_{7}$ fibration we read out the Igusa-Clebsch invariants and calculate the equation of $Y_{-}(61)$ as a double cover of $\mathbb{P}_{g, h}^{2}$.

Theorem 34. A birational model over $\mathbb{Q}$ for the Hilbert modular surface $Y_{-}(61)$ as a double cover of $\mathbb{P}_{g, h}^{2}$ is given by the equation

$$
\begin{aligned}
z^{2}=(h-1)^{4} g^{4}-2 & (h-1) h\left(h^{3}-14 h^{2}-20 h-21\right) g^{3} \\
& +h\left(h^{5}-46 h^{4}-19 h^{3}+42 h^{2}+39 h-44\right) g^{2} \\
& +2 h^{2}\left(10 h^{4}+5 h^{3}-13 h^{2}-h+12\right) g-h^{2}\left(8 h^{4}-13 h^{2}+16\right) .
\end{aligned}
$$

It is an honestly elliptic surface, with arithmetic genus 2 and Picard number 28. 
24.2. Analysis. The branch locus has genus 1 . The transformation

$$
\begin{aligned}
& g=-\frac{8 x^{3} y-34 x^{2} y+37 x y-14 y+x^{6}-10 x^{5}+44 x^{4}-89 x^{3}+98 x^{2}-58 x+14}{x^{2}(x-1)^{3}(x-7)}, \\
& h=\frac{12 x^{2} y-35 x y+26 y+3 x^{4}+11 x^{3}-61 x^{2}+74 x-26}{x^{2}\left(9 x^{2}-24 x+13\right)}
\end{aligned}
$$

converts it to the Weierstrass form

$$
y^{2}+x y=x^{3}-2 x+1,
$$

an elliptic curve of conductor 61. It is isomorphic to $X_{0}(61) /\langle w\rangle$, where $w$ is the Atkin-Lehner involution.

The Hilbert modular surface $Y_{-}(61)$ is an honestly elliptic surface, since we have an evident genus- 1 fibration over $\mathbb{P}_{h}^{1}$. Since the coefficient of $g^{4}$ is a square, we convert to the Jacobian. In Weierstrass form, we obtain

$$
\begin{aligned}
y^{2}= & x^{3}+h\left(h^{5}+14 h^{4}+23 h^{3}-102 h^{2}+88\right) x^{2} \\
& -h^{2}\left(110 h^{6}+908 h^{5}-2854 h^{4}-1028 h^{3}+4795 h^{2}+120 h-2000\right) x \\
& +h^{4}\left(1728 h^{7}+16849 h^{6}-24666 h^{5}-50145 h^{4}\right. \\
& \left.+52138 h^{3}+50406 h^{2}-29200 h-20000\right) .
\end{aligned}
$$

This elliptic surface $S$ has $\chi\left(\mathcal{O}_{S}\right)=3$, with bad fibers of type $\mathrm{I}_{0}^{*}$ at $h=0, \mathrm{I}_{7}$ at $h=\infty$, $\mathrm{I}_{2}$ at $h=1$ and at the roots of $h^{3}+13 h^{2}+24 h+16$ (which generates the cubic field of discriminant -244), and $\mathrm{I}_{3}$ at $h=-1$ and at the roots of $3 h^{4}+23 h^{3}-64 h^{2}+22 h+25$ (which generates the quartic field of discriminant $-3 \cdot 61^{2}$, a quadratic extension of $\mathbb{Q}(\sqrt{61})$ ). The trivial lattice therefore has rank 26 , leaving room for at most 4 independent sections. We find the two sections

$$
\begin{aligned}
P_{1}= & \left(h^{2}(-36 h+55), 12 h^{2}\left(3 h^{4}+23 h^{3}-64 h^{2}+22 h+25\right)\right), \\
P_{2}= & \left(-\left(36 h^{6}+444 h^{5}+472 h^{4}-1492 h^{3}-799 h^{2}+1048 h-400\right) / 61,\right. \\
& \left.4\left(15 h^{2}-2 h-5\right)\left(h^{3}+13 h^{2}+24 h+16\right)\left(3 h^{4}+23 h^{3}-64 h^{2}+22 h+25\right) / 61^{3 / 2}\right)
\end{aligned}
$$

of heights $\frac{13}{21}$ and $\frac{11}{6}$ respectively, and orthogonal to each other under the height pairing. By Oda's calculations, the Picard number is 28, and therefore the MordellWeil rank is exactly 2 . The sublattice of the Picard group generated by the above sections and the trivial lattice has discriminant $41184=2^{5} \cdot 3^{2} \cdot 11 \cdot 13$. We checked that it is 2- and 3-saturated, and therefore it must be the entire Néron-Severi lattice. Therefore the Mordell-Weil group is generated by $P_{1}$ and $P_{2}$.

24.3. Examples. Table 19 lists some points of small height and their genus-2 curves. 


\begin{tabular}{l|c}
$(g, h)$ & Sextic polynomial $f_{6}(x)$ defining the genus-2 curve $y^{2}=f_{6}(x)$ \\
\hline$\left(-\frac{3}{2}, \frac{1}{2}\right)$ & $-32 x^{6}-144 x^{5}-229 x^{4}-24 x^{3}-157 x^{2}-84 x+92$ \\
$\left(\frac{47}{72},-\frac{1}{8}\right)$ & $-10 x^{6}-42 x^{5}+39 x^{4}+728 x^{3}+489 x^{2}+1260 x-1068$ \\
$\left(-\frac{47}{12},-\frac{11}{4}\right)$ & $756 x^{6}-756 x^{5}+1953 x^{4}-282 x^{3}-327 x^{2}+1404 x-1444$ \\
$\left(-\frac{86}{9},-8\right)$ & $-348 x^{6}-972 x^{5}-2661 x^{4}-2326 x^{3}-2205 x^{2}+1260 x-140$ \\
$\left(-\frac{17}{18},-\frac{1}{2}\right)$ & $467 x^{6}+1551 x^{5}+3906 x^{4}+3027 x^{3}-495 x^{2}-1800 x+400$ \\
$\left(\frac{15}{4}, 5\right)$ & $-325 x^{6}+1410 x^{5}+1045 x^{4}-3993 x^{3}-2636 x^{2}+3456 x+2143$ \\
$\left(-\frac{69}{58},-\frac{20}{29}\right)$ & $-128 x^{6}+96 x^{5}-2519 x^{4}+4362 x^{3}+1321 x^{2}+456 x+32$ \\
$\left(\frac{31}{45}, \frac{14}{5}\right)$ & $-204 x^{6}-108 x^{5}+2553 x^{4}-946 x^{3}-4683 x^{2}-360 x+1200$ \\
$\left(1, \frac{4}{3}\right)$ & $-188 x^{6}-1812 x^{5}-4707 x^{4}-130 x^{3}+6189 x^{2}-1620 x+108$ \\
$\left(\frac{13}{18},-\frac{1}{2}\right)$ & $-612 x^{6}+3708 x^{5}-6501 x^{4}+5656 x^{3}+693 x^{2}-318 x-1126$ \\
$\left(-\frac{29}{6},-\frac{29}{8}\right)$ & $-148 x^{6}+2472 x^{5}-1481 x^{4}+5001 x^{3}-6980 x^{2}+1425 x-6625$ \\
$\left(-\frac{37}{18},-\frac{1}{2}\right)$ & $-16 x^{6}-264 x^{5}+477 x^{4}-2268 x^{3}+4029 x^{2}-6156 x+10372$ \\
$\left(\frac{23}{84},-\frac{1}{3}\right)$ & $-4671 x^{6}-6660 x^{5}-10362 x^{4}+11195 x^{3}+1287 x^{2}+1590 x+7621$ \\
$\left(\frac{5}{6}, \frac{5}{3}\right)$ & $821 x^{6}-1896 x^{5}-4922 x^{4}+8588 x^{3}+11341 x^{2}-7674 x-8247$ \\
$\left(-\frac{23}{2},-10\right)$ & $2716 x^{6}+84 x^{5}+7107 x^{4}+10642 x^{3}+4803 x^{2}+13764 x+10204$ \\
$\left(\frac{65}{18}, \frac{5}{2}\right)$ & $-3756 x^{6}-12012 x^{5}+9297 x^{4}+18116 x^{3}-10335 x^{2}-7560 x+3600$ \\
\hline
\end{tabular}

Table 19. Some rational points $(g, h)$ of small height on the surface of Theorem 34 and the corresponding genus- 2 curves.

Next, we list some rational curves on the surface. The specialization $h=-1$ gives a rational curve, but the curves of genus 2 corresponding to the points on this curve have Jacobians with endomorphism ring larger than just $\mathcal{O}_{61}$ (they are isogenous to the symmetric squares of elliptic curves). The section $P_{1}$ gives the rational curve $g=\left(3 h^{2}-7 h+5\right) /(3(h-1))$, for which the Brauer obstruction vanishes identically, yielding a 1-parameter family of genus- 2 curves whose Jacobian have real multiplication by $\mathcal{O}_{61}$.

\section{Discriminant 65}

25.1. Parametrization. We start with an elliptic surface with $E_{7}, A_{4}$ and $A_{4}$ fibers at $t=\infty, 0,1$ respectively, and a section of height $\frac{65}{50}=\frac{13}{10}=4-\frac{3}{2}-\frac{2 \cdot 3}{5}$. The Weierstrass equation of such a family is

$$
\begin{aligned}
y^{2}=x^{3} & +\left(a_{0}(1-t)+a_{1} t(1-t)+t^{2}\right) x^{2} \\
& +2 t^{2}(t-1) e\left(b_{0}(1-t)+b_{1} t(1-t)+t^{2}\right) x+e^{2} t^{4}(t-1)^{2}\left(c_{0}(1-t)+t\right),
\end{aligned}
$$

with

$$
a_{0}=\left(s^{2}-5\right)^{2}\left(2 r s^{2}+s+2 r\right)^{2} / 4,
$$




$$
\begin{aligned}
a_{1} & =4\left(5 s^{6}-8 s^{4}-7 s^{2}-6\right) r^{2}+4 s\left(5 s^{4}-13 s^{2}-4\right) r+5 s^{4}-18 s^{2}+5, \\
b_{0} & =\left(s^{2}-5\right)\left(2 r s^{2}+s+2 r\right)\left(2 r s^{4}+s^{3}+4 r s^{2}+s-6 r\right) / 4, \\
b_{1} & =8\left(s^{2}-1\right)\left(s^{2}+1\right)^{2} r^{2}+8 s\left(s^{4}-1\right) r+2 s^{4}-2 s^{2}+1, \\
c_{0} & =\left(2 r s^{4}+s^{3}+4 r s^{2}+s-6 r\right)^{2} / 4, \\
e & =-(s-1)(s+1)\left(2 r s^{2}-4 r s+s+2 r-2\right)\left(2 r s^{2}+4 r s+s+2 r+2\right) .
\end{aligned}
$$

To describe an elliptic fibration with $E_{8}$ and $E_{7}$ fibers, we identify the class of an $E_{8}$ fiber and move by a 2-neighbor step to an $E_{8} A_{4} A_{2}$ fibration.

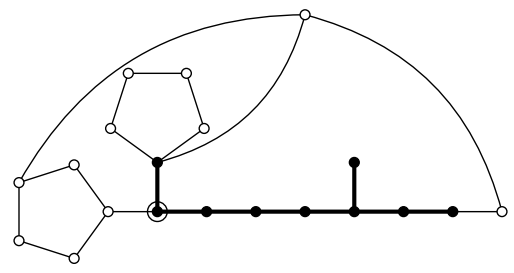

The new elliptic fibration has Mordell-Weil rank 2, and we compute two generators $P$ and $Q$, each of height $\frac{32}{15}=4-\frac{2}{3}-\frac{6}{5}$, with intersection pairing $\frac{7}{15}$. We draw $P$ in the figure below, as well as the class of an $A_{7}$ fiber $F^{\prime}$. Because $Q \cdot F^{\prime}=1$, the new fibration has a section.

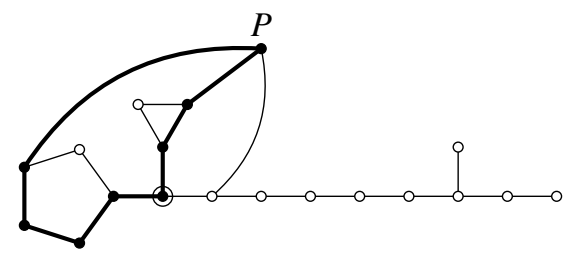

The new elliptic fibration has $A_{7}$ and $E_{8}$ fibers, and a section $P^{\prime}$ of height $\frac{65}{8}=4+2 \cdot 3-3 \cdot \frac{5}{8}$. We now go to $E_{8} E_{7}$ via a 2-neighbor step. Note that the section $P^{\prime}$ intersects the new $E_{7}$ fiber $F^{\prime \prime}$ in 7 , while the remaining component of the $A_{7}$ fiber intersects $F^{\prime \prime}$ in 2. Since these are coprime, the genus-1 fibration defined by $F^{\prime \prime}$ has a section.

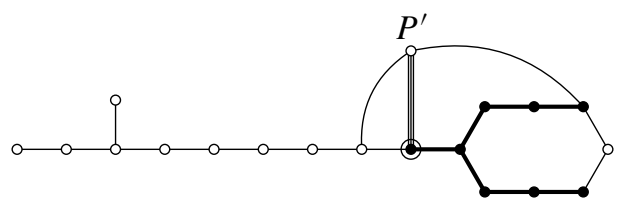

We now read out the Igusa-Clebsch invariants, and compute the equation of $Y_{-}(65)$ as a double cover of $\mathbb{P}_{r, s}^{2}$. It is branched over the locus where the K3 surfaces acquire an extra $\mathrm{I}_{2}$ fiber. 
Theorem 35. A birational model over $\mathbb{Q}$ for the Hilbert modular surface $Y_{-}(65)$ as a double cover of $\mathbb{P}_{r, s}^{2}$ is given by the equation

$$
\begin{aligned}
& z^{2}=-16\left(s^{4}+2 s^{2}+13\right)^{2}\left(4 s^{6}+3 s^{4}-10 s^{2}-13\right) r^{4} \\
&- 32 s\left(4 s^{12}+15 s^{10}+127 s^{8}-10 s^{6}-494 s^{4}-1253 s^{2}-949\right) r^{3} \\
&-8\left(12 s^{12}+33 s^{10}+408 s^{8}-898 s^{6}-2672 s^{4}-2023 s^{2}+404\right) r^{2} \\
&-8 s\left(4 s^{10}+7 s^{8}+149 s^{6}-627 s^{4}-641 s^{2}+148\right) r \\
&-\left(4 s^{10}+3 s^{8}+166 s^{6}-997 s^{4}+328 s^{2}-80\right) .
\end{aligned}
$$

It is an honestly elliptic surface, with arithmetic genus 2 and Picard number 28.

25.2. Analysis. This is an honestly elliptic surface, with the extra involution $\iota$ : $(r, s, z) \mapsto(-r,-s, z)$ corresponding to the factorization $65=5 \cdot 13$.

The branch locus is a curve of genus 1 , isomorphic to the elliptic curve

$$
y^{2}+x y=x^{3}-x
$$

of conductor 65. For lack of space we do not write down the explicit isomorphism here, relegating the relevant formulae to the online supplement. This elliptic curve is isomorphic to the quotient of $X_{0}(65)$ by the Atkin-Lehner involution $w_{65}$.

We were unable to find a section of this genus- 1 fibration. However, for purposes of analyzing the Picard number, we study the Jacobian of this elliptic curve over $\mathbb{Q}(s)$, given by

$$
\begin{aligned}
y^{2}=x^{3}+\left(8 s^{6}+\right. & \left.13 s^{4}-106 s^{2}+101\right) x^{2} \\
+ & \left(16 s^{12}+52 s^{10}-564 s^{8}+1416 s^{6}-1624 s^{4}+900 s^{2}-196\right) x .
\end{aligned}
$$

This has reducible fibers of type $\mathrm{I}_{8}$ at $s= \pm 1, \mathrm{I}_{4}$ at $s=\infty, \mathrm{I}_{3}$ at $s= \pm \frac{\sqrt{13}}{3}$, and $\mathrm{I}_{2}$ at the four roots of $4 s^{4}+29 s^{2}-49$ (a dihedral extension containing $\sqrt{65}$ ). The trivial lattice therefore has rank 27, leaving room for Mordell-Weil rank at most 3. There is the obvious 2-torsion section $(0,0)$, and we find a non-torsion section of height $\frac{2}{3}$ :

$$
\begin{aligned}
& P=\left((73+9 \mu) / 2\left(s^{2}-1\right)^{2}\left(s^{2}+(29 / 8-5 / 8 \mu)\right),\right. \\
& \left.(657+81 \mu) / 2 s\left(s^{2}-1\right)^{2}\left(s^{2}-13 / 9\right)\left(s^{2}+(29 / 8-5 / 8 \mu)\right)\right)
\end{aligned}
$$

with $\mu=\sqrt{65}$. Analysis of the quotient by $\iota$, and its twist, shows that the MordellWeil rank is exactly 1 . Therefore the Picard number of $Y_{-}(65)$ is 28 . The discriminant of the sublattice of the Néron-Severi group generated by the trivial lattice and these two sections is $6144=2^{11} \cdot 3$. We checked that it is 2 -saturated, and so it equals the entire Néron-Severi lattice. Therefore the sections above generate the Mordell-Weil group. 
The quotient by the involution $\iota$ is given by the equation

$$
\begin{aligned}
w^{2}=-16 t^{2}\left(t^{2}+2 t\right. & +13)^{2}\left(4 t^{3}+3 t^{2}-10 t-13\right) r^{4} \\
& -32 t^{2}\left(4 t^{6}+15 t^{5}+127 t^{4}-10 t^{3}-494 t^{2}-1253 t-949\right) r^{3} \\
& -8 t\left(12 t^{6}+33 t^{5}+408 t^{4}-898 t^{3}-2672 t^{2}-2023 t+404\right) r^{2} \\
& -8 t\left(4 t^{5}+7 t^{4}+149 t^{3}-627 t^{2}-641 t+148\right) r \\
- & \left(4 t^{5}+3 t^{4}+166 t^{3}-997 t^{2}+328 t-80\right),
\end{aligned}
$$

where $t=s^{2}$. Once again we study the Jacobian elliptic fibration: it has the equation

$$
\begin{aligned}
y^{2}=x^{3}+\left(8 t^{4}+\right. & \left.13 t^{3}-106 t^{2}+101 t\right) x^{2} \\
& +\left(16 t^{8}+52 t^{7}-564 t^{6}+1416 t^{5}-1624 t^{4}+900 t^{3}-196 t^{2}\right) x .
\end{aligned}
$$

This is an elliptic K3 surface with bad fibers of type $\mathrm{I}_{0}^{*}$ at $t=0, \mathrm{I}_{8}$ at $t=1, \mathrm{I}_{3}$ at $t=\frac{13}{9}$, and $\mathrm{I}_{2}$ at $t=\infty$ and $t=(-29 \pm 5 \sqrt{65}) / 8$. Therefore the trivial lattice has rank 18, and the Mordell-Weil rank can be at most 2. As before we have a 2-torsion

\begin{tabular}{l|c}
$(r, s)$ & Sextic polynomial $f_{6}(x)$ defining the genus-2 curve $y^{2}=f_{6}(x)$ \\
\hline$\left(\frac{40}{41},-\frac{1}{5}\right)$ & $-396 x^{6}+216 x^{5}+281 x^{4}-889 x^{3}+50 x^{2}+939 x+315$ \\
$\left(-\frac{40}{41}, \frac{1}{5}\right)$ & $-648 x^{5}+3015 x^{4}-422 x^{3}-4369 x^{2}+2216 x-752$ \\
$\left(\frac{35}{136}, \frac{1}{5}\right)$ & $-72 x^{6}+969 x^{5}-3509 x^{4}+847 x^{3}+9373 x^{2}+816 x-3724$ \\
$\left(-\frac{40}{143},-\frac{2}{5}\right)$ & $-240 x^{6}-384 x^{5}+695 x^{4}+2724 x^{3}+5543 x^{2}-10992 x-2736$ \\
$\left(\frac{2}{15},-2\right)$ & $-16200 x^{5}+1125 x^{4}-8972 x^{3}-30493 x^{2}+14186 x-18974$ \\
$\left(\frac{40}{143}, \frac{2}{5}\right)$ & $-4368 x^{6}+420 x^{5}+28144 x^{4}-13235 x^{3}-35846 x^{2}+10080 x+14112$ \\
$\left(-\frac{5}{64}, \frac{7}{5}\right)$ & $800 x^{6}+6480 x^{5}+19405 x^{4}+35306 x^{3}-39491 x^{2}-2688 x-48$ \\
$\left(-\frac{49}{197}, \frac{1}{2}\right)$ & $5088 x^{6}-48648 x^{5}+85307 x^{4}+9352 x^{3}-59071 x^{2}-15690 x+730$ \\
$\left(\frac{49}{197},-\frac{1}{2}\right)$ & $546 x^{6}+9798 x^{5}+24115 x^{4}-25228 x^{3}-98531 x^{2}+58920 x+38880$ \\
$\left(-\frac{35}{136},-\frac{1}{5}\right)$ & $2744 x^{6}-22344 x^{5}-45297 x^{4}-16942 x^{3}+100440 x^{2}+89910 x-72900$ \\
$\left(\frac{5}{64},-\frac{7}{5}\right)$ & $-332100 x^{6}+344220 x^{5}-54545 x^{4}+106126 x^{3}-68117 x^{2}+3528 x-16464$ \\
$\left(-\frac{2}{15}, 2\right)$ & $-216000 x^{6}+506400 x^{5}-283195 x^{4}-70483 x^{3}+13883 x^{2}+3456 x+300$ \\
$\left(-\frac{1}{65}, \frac{1}{2}\right)$ & $-366600 x^{6}-2197788 x^{5}-64538 x^{4}+11447529 x^{3}+133360 x^{2}$ \\
$\left(\frac{1}{65},-\frac{1}{2}\right)$ & $-412287975 x^{6}-3236837061 x^{5}+5479876697 x^{4}+3156545763 x^{3}$ \\
& $+1177706300 x^{2}-7413585000 x-1103500000$ \\
\hline
\end{tabular}

Table 20. Some rational points $(r, s)$ of small height on the surface of Theorem 35 and the corresponding genus-2 curves. 
point $P_{1}=(0,0)$. We also find a non-torsion point

$$
\begin{aligned}
P_{2}=\left((73-9 \mu) t(t-1)^{2}(8 t+\right. & 29+5 \mu) / 16, \\
& \left.(-73+9 \mu) t^{2}(t-1)^{2}(9 t-13)(8 t+29+5 \mu) / 16\right)
\end{aligned}
$$

of height $\frac{1}{3}$, with $\mu=\sqrt{65}$ as before. Therefore, the Picard number is at least 19 , and point counting modulo 11 and 23 shows that the Picard number must be 19 . We verified by checking 2-saturation that the sections $P_{1}$ and $P_{2}$ and the trivial lattice span the Néron-Severi group, which has discriminant 64.

We next analyze the quotient by $\iota^{\prime}:(r, s, z) \mapsto(-r,-s,-z)$, which is the quadratic twist of the elliptic K3 surface above by $\sqrt{t}$ :

$$
\begin{aligned}
y^{2}=x^{3}+\left(8 t^{3}+13 t^{2}\right. & -106 t+101) x^{2} \\
+ & \left(16 t^{6}+52 t^{5}-564 t^{4}+1416 t^{3}-1624 t^{2}+900 t-196\right) x .
\end{aligned}
$$

This is also an elliptic K3 surface, with reducible fibers of type $\mathrm{I}_{2}^{*}$ at $t=\infty$, $\mathrm{I}_{8}$ at $t=1, \mathrm{I}_{3}$ at $t=\frac{13}{9}$, and $\mathrm{I}_{2}$ at $t=(-29 \pm 5 \sqrt{65}) / 8$. The trivial lattice has rank 19 . Again, point counting modulo 11 and 23 shows that the Picard number is 19, and the Mordell-Weil group therefore has rank 0 , with only the 2-torsion section $(0,0)$.

25.3. Examples. Table 20 on the previous page lists some points of small height and their genus- 2 curves.

\section{Discriminant 69}

26.1. Parametrization. Start with an elliptic $\mathrm{K} 3$ surface with fibers of type $E_{6}, A_{8}$ and $A_{1}$ and a section of height $\frac{69}{54}=\frac{23}{18}=4-\frac{1}{2}-\frac{20}{9}$. We can write down the Weierstrass equation of this family as

$$
y^{2}=x^{3}+\left(a_{0}+a_{1} t+a_{2} t^{2}\right) x^{2}+t^{3}\left(b_{0}+b_{1} t+b_{2} t^{2}\right) x+t^{6}\left(c_{0}+c_{1} t+c_{2} t^{2}\right),
$$

with

$$
\begin{array}{rlrl}
a_{0}=\frac{1}{4}\left(\sigma_{2}-\sigma_{1}+2\right)^{2}, & a_{1}=\frac{1}{2}\left(\sigma_{2}^{2}-\sigma_{2}\left(\sigma_{1}-4\right)-2\left(\sigma_{1}-1\right)\right), \\
a_{2}=\frac{1}{4}\left(\sigma_{2}^{2}+4 \sigma_{2}-8\right), & b_{0}=\frac{1}{2} \sigma_{2}\left(\sigma_{2}-\sigma_{1}+2\right)^{2}, \\
b_{1}=\frac{1}{2}\left(\sigma_{2}^{3}-\sigma_{2}^{2}\left(\sigma_{1}-4\right)-\sigma_{1}^{2}-\sigma_{2} \sigma_{1}+2 \sigma_{1}\right), & b_{2}=\frac{1}{2}\left(\sigma_{2}\left(\sigma_{1}-6\right)+2\left(\sigma_{1}-1\right)\right), \\
c_{0}=\frac{1}{4} \sigma_{2}^{2}\left(\sigma_{2}-\sigma_{1}+2\right)^{2}, & c_{1}=\frac{1}{2} \sigma_{2}\left(\sigma_{1}-2\right)\left(\sigma_{2}-\sigma_{1}\right), & c_{2}=\frac{1}{4}\left(\sigma_{1}^{2}-4 \sigma_{2}\right),
\end{array}
$$

where

$$
\sigma_{1}=r+s, \quad \sigma_{2}=r s
$$


First we find the class of another $E_{6}$ fiber below and go to that elliptic fibration via a 2-neighbor step.

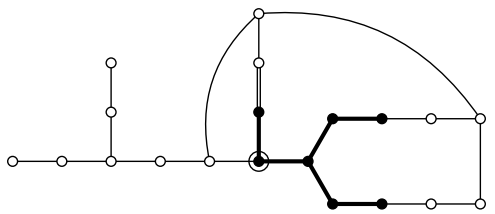

The resulting elliptic fibration has $E_{6}, E_{6}$ and $A_{3}$ fibers, and a section of height $\frac{23}{12}=4-\frac{4}{3}-\frac{3}{4}$. Now we find the class of an $A_{7}$ fiber in the diagram below.

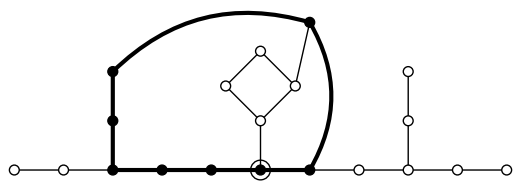

The resulting fibration has $A_{7}, A_{5}, A_{2}$ and $A_{1}$ fibers, a 2-torsion section, and a non-torsion section $P$ of height $\frac{69}{72}=\frac{23}{24}=4-\frac{2}{3}-\frac{3 \cdot 3}{6}-\frac{1 \cdot 7}{8}$. We next identify the class $F^{\prime}$ of an $E_{7}$ fiber, and go to it via a 2-neighbor step. Note that $P \cdot F^{\prime}=1$, so the new fibration has a section.

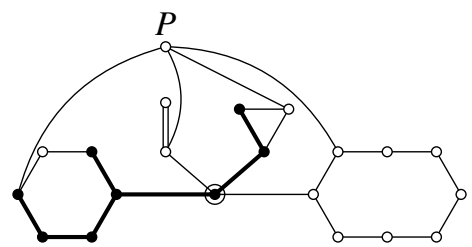

The new elliptic fibration has $E_{7}, A_{7}$ and $A_{1}$ fibers, a 2-torsion section $Q^{\prime}$, and a non-torsion section $P^{\prime}$ of height $\frac{69}{8}=4+2 \cdot 3-\frac{1}{2}-\frac{7}{8}$. We identify a fiber $F^{\prime \prime}$ of type $E_{7}$ below.

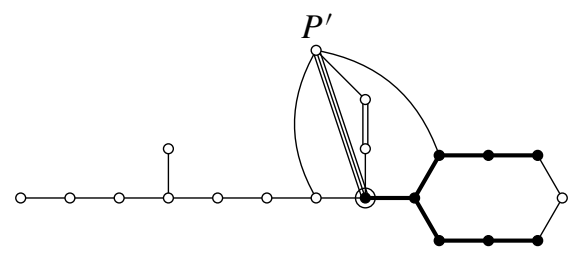

Note that $P^{\prime} \cdot F^{\prime \prime}=2 \cdot 3+3=9$, while the remaining component of the $A_{7}$ fiber intersects $F^{\prime \prime}$ with multiplicity 2 . Therefore the new elliptic fibration has a section. Converting to the Jacobian, we read out the Weierstrass coefficients of the $E_{8} E_{7}$ form, which give us the Igusa-Clebsch invariants. 
Theorem 36. A birational model over $\mathbb{Q}$ for the Hilbert modular surface $Y_{-}(69)$ as a double cover of $\mathbb{P}_{r, s}^{2}$ is given by the equation

$$
\begin{aligned}
z^{2}=(r & -1)^{2} r^{4} s^{6}-2(r-1) r^{2}\left(r^{3}+13 r^{2}-37 r+22\right) s^{5} \\
& +\left(r^{6}+100 r^{5}-439 r^{4}+640 r^{3}-357 r^{2}+72 r-16\right) s^{4} \\
& -2\left(59 r^{5}-320 r^{4}+590 r^{3}-436 r^{2}+133 r-32\right) s^{3} \\
& +\left(44 r^{5}-357 r^{4}+872 r^{3}-830 r^{2}+314 r-83\right) s^{2} \\
& +2\left(36 r^{4}-133 r^{3}+157 r^{2}-65 r+19\right) s-16 r^{4}+64 r^{3}-83 r^{2}+38 r-11 .
\end{aligned}
$$

It is a surface of general type.

26.2. Analysis. This is a surface of general type, with an extra involution $(r, s, z) \mapsto$ $(s, r, z)$, corresponding to $69=3 \cdot 23$.

The branch locus is a curve of genus 2 ; the transformation

$$
\begin{aligned}
& r=-\frac{x^{3} y+x^{2} y-y-3 x^{6}-4 x^{5}+x^{4}+6 x^{3}-2 x^{2}-2 x+1}{2 x^{2}\left(x^{2}+x-1\right)^{2}}, \\
& s=\frac{x^{3} y+x^{2} y-y+3 x^{6}+4 x^{5}-x^{4}-6 x^{3}+2 x^{2}+2 x-1}{2 x^{2}\left(x^{2}+x-1\right)^{2}}
\end{aligned}
$$

converts it into Weierstrass form

$$
y^{2}=\left(x^{3}+x^{2}-1\right)\left(5 x^{3}-7 x^{2}+4 x-1\right) .
$$

It is isomorphic to $X_{0}(69) /\langle w\rangle$, where $w$ is the Atkin-Lehner involution.

The quotient surface is (with $m=r+s, n=r s$ )

$$
\begin{aligned}
z^{2}=-16 m^{4}+4\left(11 n^{2}+18 n\right. & +16) m^{3}+\left(n^{4}-118 n^{3}-357 n^{2}-202 n-83\right) m^{2} \\
& -2\left(n^{5}-50 n^{4}-254 n^{3}-328 n^{2}-61 n-19\right) m \\
& +n^{6}-26 n^{5}-203 n^{4}-466 n^{3}-330 n^{2}+36 n-11 .
\end{aligned}
$$

The substitution $m=n+k$ makes the right-hand side quartic in $n$, with highest coefficient a square. Converting to the Jacobian, we get (after some Weierstrass transformations and change of the parameter on the base) the elliptic K3 surface

$$
\begin{array}{r}
y^{2}=x^{3}-\left(88 t^{3}+15 t^{2}+6 t-1\right) x^{2}+8 t^{3}\left(250 t^{3}+57 t^{2}+45 t-8\right) x \\
+16 t^{5}\left(1125 t^{3}+552 t^{2}+208 t-36\right) .
\end{array}
$$

This has fibers of type $\mathrm{I}_{5}$ at $t=0, \mathrm{I}_{0}^{*}$ at $t=\infty, \mathrm{I}_{2}$ at $t=(-3 \pm 2 \sqrt{3}) / 4$, and $\mathrm{I}_{3}$ at the roots of $25 t^{3}+17 t^{2}+2 t-1$ (which generates the cubic field of discriminant -23). The trivial lattice has rank 18 , leaving room for at most two independent sections. We find the non-torsion section

$$
P_{1}=\left(4 t(1-t), 4 t\left(25 t^{3}+17 t^{2}+2 t-1\right)\right)
$$


of height $\frac{1}{5}$. Counting points modulo 7 and 13 then shows that the Picard number must be exactly 19 . Therefore, the discriminant of the sublattice spanned by $P_{1}$ and the trivial lattice is $432=2^{4} \cdot 3^{3}$. Looking at the contributions to the Néron-Tate height from the fiber configuration, one easily sees that there cannot be any 2- or 3-torsion. Similarly, it is impossible to have a section of height $\frac{1}{20}$ or $\frac{1}{45}$. Therefore, this sublattice must be the entire Néron-Severi lattice, and $P_{1}$ is a generator of the Mordell-Weil group.

26.3. Examples. Table 21 lists some points of small height and their genus- 2 curves.

\begin{tabular}{|c|c|}
\hline & \\
\hline & $-144 x^{6}-336 x^{5}+491 x^{4}-274 x^{3}+4919 x^{2}-23076 x-6476$ \\
\hline & $-108456 x^{6}+89940 x^{5}+3518 x^{4}+11915 x^{3}+29021 x^{2}-40515 x+2841$ \\
\hline & $-7146 x^{6}+26076 x^{5}+26698 x^{4}-128487 x^{3}-87881 x^{2}+140967 x+106899$ \\
\hline & $-205648 x^{6}-71112 x^{5}+4931 x^{4}-3219 x^{3}-1369 x^{2}+336 x+64$ \\
\hline & $-47792 x^{6}+212184 x^{5}-134731 x^{4}-131082 x^{3}+58025 x^{2}+39900 x+3500$ \\
\hline & $111132 x^{6}+308700 x^{5}+150199 x^{4}-166350 x^{3}-85877 x^{2}+37080 x+3208$ \\
\hline & $\begin{array}{r}-203124 x^{6}+537156 x^{5}-1147529 x^{4}-958036 x^{3} \\
-185681 x^{2}+583356 x-97236\end{array}$ \\
\hline ( & $\begin{array}{r}-4138876 x^{6}-12791196 x^{5}-14043627 x^{4}-25 \\
-2332\end{array}$ \\
\hline 3$)$ & $\begin{array}{r}-4774900 x^{6}+11612125 x^{5}-1487685 x^{4}-1 \\
+29039\end{array}$ \\
\hline$\left.\frac{13}{9}\right)$ & $\begin{array}{r}-1749188 x^{6}+9004404 x^{5}-5544841 x^{4}- \\
-3645\end{array}$ \\
\hline & $\begin{array}{r}1490720 x^{6}+34810248 x^{5}+203477725 \\
-3925\end{array}$ \\
\hline & $\begin{array}{r}354444 x^{6}+2968308 x^{5}-37732823 x^{4}+471 \\
+22332350\end{array}$ \\
\hline$\left.-\frac{25}{3}, \frac{5}{4}\right)$ & $\begin{array}{r}-28660432 x^{6}-9277032 x^{5}-367100597 x^{4}+ \\
-1142233133 x\end{array}$ \\
\hline & $953161100 x^{6}-1709768900 x^{5}+5715$ \\
\hline & $\begin{aligned} 6865596 x^{6}-82041816 x^{5}+ & 58608103 x^{4}+ \\
& +92125689\end{aligned}$ \\
\hline & $\begin{array}{r}-125838448 x^{6}+33513120 x^{5}-1068122125 x^{4}+1 \\
-2407159591 x^{2}+462\end{array}$ \\
\hline
\end{tabular}

Table 21. Some rational points $(r, s)$ of small height on the surface of Theorem 36 and the corresponding genus- 2 curves. 
By pulling back some of the low-height sections of the quotient, we produce curves of low genus on $Y_{-}$(69). Since $r+s=m$ and $r s=n$, the appropriate condition is that $m^{2}-4 n=(r-s)^{2}$ be a square (then we can take a square root and solve for $r, s)$. In other words, $(n+k)^{2}-4 n$ must be a square. The section $P_{1}$ is given by $n=-\left(5 k^{2}-17 k+15\right) /(k-2)$; it gives rise to a genus- 1 curve

$$
y^{2}=16 k^{4}-100 k^{3}+237 k^{2}-254 k+105,
$$

which has rational points (for instance, at infinity). It is therefore an elliptic curve, and we calculate that it has conductor 1711 (prime) and Mordell-Weil group $\mathbb{Z}^{2}$.

Similarly, the section $-P_{1}$ is defined by $n=-\left(25 k^{2}-92 k+89\right) /(10 k-21)$. It also gives rise to a genus- 1 curve

$$
y^{2}=225 k^{4}-1130 k^{3}+1931 k^{2}-1350 k+445
$$

with rational points (as at $k=\infty$ ). It is an elliptic curve of conductor $50435=$ $5 \cdot 7 \cdot 11 \cdot 131$, with trivial torsion and rank 1 .

\section{Discriminant 73}

27.1. Parametrization. Start with a K 3 elliptic surface with fibers of type $A_{6}, A_{9}$ and a section of height $\frac{73}{70}=4-\frac{21}{10}-\frac{6}{7}$. The Weierstrass equation is

$$
\begin{aligned}
y^{2}=x^{3}+\left(a_{0}+a_{1} t+a_{2} t^{2}\right. & \left.+a_{3} t^{3}+a_{4} t^{4}\right) x^{2} \\
+ & 2 \mu t\left(b_{0}+b_{1} t+b_{2} t^{2}+b_{3} t^{3}\right) x+\mu^{2} t^{2}\left(c_{0}+c_{1} t+c_{2} t^{2}\right),
\end{aligned}
$$

with

$$
\begin{aligned}
& \mu=-2 s^{4}(s+2 r)^{2}, \quad a_{0}=s^{2}(r s-2 r-2)^{2}, \quad a_{4}=16 r^{2}(r+1)^{2}, \\
& b_{0}=s(r s-2 r-2)^{2}, \quad b_{3}=4 r(r+1)^{2}(s+2 r+2), \quad c_{0}=(r s-2 r-2)^{2} \text {, } \\
& c_{2}=(r+1)^{2}(s+2 r+2)^{2} \text {, } \\
& a_{3}=8 r(r+1)\left((r+1) s^{2}+2(4 r+1) s+4 r(r-1)\right) \text {, } \\
& c_{1}=-2(s+2 r+2)\left(s^{2}+(r+2)(r-1) s-2\left(r^{2}-1\right)\right) \text {, } \\
& b_{1}=-3 s^{4}-2 r(r+5) s^{3}-2\left(r^{3}+4 r^{2}-2 r-2\right) s^{2}-8(r+1) s+8 r(r+1)^{2} \text {, } \\
& a_{1}=-2 s\left(3 s^{4}+r(r+11) s^{3}+2\left(5 r^{2}-r-1\right) s^{2}+4(r+1)\left(r^{2}+1\right) s-8 r(r+1)^{2}\right) \text {, } \\
& b_{2}=(r+1)^{2} s^{3}+2\left(r^{3}+7 r^{2}+6 r+2\right) s^{2} \\
& +4\left(4 r^{3}+8 r^{2}+7 r+1\right) s+8(r-1) r(r+1)(r+2), \\
& a_{2}=(r+1)^{2} s^{4}+4\left(4 r^{2}+3 r+1\right) s^{3}+4\left(4 r^{3}+10 r^{2}+10 r+1\right) s^{2} \\
& +16 r(r+1)(r-2) s+16 r^{2}(r+1)^{2} \text {. }
\end{aligned}
$$


We first identify the class of an $E_{8}$ fiber, and perform a 3-neighbor step to move to an elliptic fibration with $E_{8}$ and $A_{7}$ fibers.

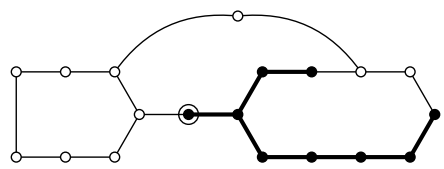

This fibration has a section of height $\frac{73}{8}=4+2 \cdot 3-\frac{7}{8}$. Next, we locate an $E_{7}$ fiber and compute a 2-neighbor step to go to a fibration with $E_{8}$ and $E_{7}$ fibers.

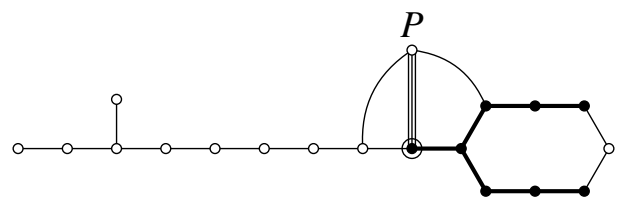

The intersection number of the new fiber $F^{\prime}$ with the remaining component of the $A_{7}$ fiber is 2 and with the section $P$ is 9 . Therefore, the new genus- 1 fibration defined by $F^{\prime}$ has a section since 9 and 2 are coprime.

Theorem 37. A birational model over $\mathbb{Q}$ for the Hilbert modular surface $Y_{-}(73)$ as a double cover of $\mathbb{P}_{r, s}^{2}$ is given by the equation

$$
\begin{aligned}
& z^{2}=16(s-2)^{2} r^{4}+8(s-2)\left(17 s^{3}-52 s^{2}+36 s-8\right) r^{3} \\
& +\left(s^{6}+56 s^{5}-384 s^{4}+448 s^{3}+432 s^{2}-512 s+64\right) r^{2} \\
& \quad+2 s(s+2)\left(s^{4}-34 s^{3}+108 s^{2}-64 s+16\right) r+s^{2}(s+2)^{4} .
\end{aligned}
$$

It is an honestly elliptic surface, with arithmetic genus 2 and Picard number 28.

27.2. Analysis. The branch locus is a curve of genus 2 ; the transformation of coordinates

$$
\begin{aligned}
& r=\frac{3 x^{3} y-3 x y+y-x^{6}-2 x^{5}-4 x^{4}-3 x^{2}+4 x-1}{2 x^{2}\left(x^{2}+2 x-1\right)^{2}}, \\
& s=-2 \frac{\left(2 x y-y-x^{3}-7 x^{2}+3 x\right)}{(x+1)^{2}\left(x^{2}+2 x-1\right)}
\end{aligned}
$$

converts it to Weierstrass form

$$
y^{2}=x^{6}+4 x^{5}+2 x^{4}-6 x^{3}+x^{2}-2 x+1 .
$$

This is a genus- 2 curve, isomorphic to the quotient of $X_{0}(73)$ by the Atkin-Lehner involution.

The Hilbert modular surface $Y_{-}(73)$ is an elliptic surface. Since the coefficient of $r^{4}$ is a square, this genus- 1 curve over $\mathbb{P}_{s}^{1}$ has a section. Computing the Jacobian, 
we get the following Weierstrass equation after a change of parameter on the base and some simple Weierstrass transformations:

$$
\begin{aligned}
y^{2}=x^{3}-( & \left.83 t^{6}-316 t^{5}+390 t^{4}-158 t^{3}-21 t^{2}+22 t-1\right) x^{2} \\
& +8(t-1)^{4} t^{3}\left(287 t^{5}-1040 t^{4}+960 t^{3}-73 t^{2}-217 t+67\right) x \\
& -16(t-1)^{7} t^{6}\left(1323 t^{5}-5887 t^{4}+7110 t^{3}-1426 t^{2}-2201 t+1033\right) .
\end{aligned}
$$

This is an honestly elliptic surface with $\chi=3$. It has reducible fibers of type $\mathrm{I}_{7}$ at $t=1, \mathrm{I}_{6}$ at $t=0, \mathrm{I}_{5}$ at $t=\infty, \mathrm{I}_{3}$ at $t=(-5 \pm \sqrt{73}) / 4$, and $\mathrm{I}_{2}$ at $t=-1$ and $(13 \pm \sqrt{73}) / 24$. The trivial lattice therefore has rank 24 , leaving room for Mordell-Weil rank at most 6.

We find the following four independent sections.

$$
\begin{aligned}
P_{1}= & \left(4 t^{3}(t-1)^{2}(9 t-7), 4 t^{3}(t-1)^{2}(t+1)(3 t-2)\left(2 t^{2}+5 t-6\right)\right), \\
P_{2}= & \left(4 t^{3}(t-1)\left(7 t^{2}-17 t+8\right), 4 t^{3}(t-1)(t+1)\left(9 t^{2}-13 t+5\right)\right), \\
P_{3}= & \left(4 t^{2}(t-1)^{3}(19 t-16), 4 t^{2}(t-1)^{3}\left(7 t^{2}-12 t+8\right)\left(12 t^{2}-13 t+2\right)\right), \\
P_{4}= & \left(4 t^{3}\left(7 t^{3}-24 t^{2}-2 \mu t+42 t-2 \mu+9\right),\right. \\
& \left.(-13+\mu) t^{3}(t+1)(-24 t+13+\mu)(-17-16 t+3 \mu)(-4 t-5+\mu) / 192\right)
\end{aligned}
$$

(where $\mu=\sqrt{73}$ ), with nondegenerate height pairing matrix

$$
\left(\begin{array}{cccc}
\frac{26}{21} & \frac{5}{7} & -\frac{1}{7} & -\frac{2}{3} \\
\frac{5}{7} & \frac{68}{35} & \frac{11}{7} & -\frac{1}{5} \\
-\frac{1}{7} & \frac{11}{7} & \frac{41}{21} & \frac{1}{2} \\
-\frac{2}{3} & -\frac{1}{5} & \frac{1}{2} & \frac{49}{30}
\end{array}\right) .
$$

Therefore, the Mordell-Weil rank is at least 4. From Oda's calculations [1982, p. 109], the Picard number is 28 , so the Mordell-Weil rank is $28-24=4$ and our sections generate a subgroup of finite index in the full Mordell-Weil group. The sublattice of the Néron-Severi lattice generated by the trivial lattice and these sections has discriminant $3916=2^{2} \cdot 11 \cdot 89$. We checked that this sublattice is 2 -saturated, and therefore it is the entire Néron-Severi lattice.

27.3. Examples. Table 22 lists some points of small height and their genus- 2 curves.

We get many curves of genus 0 on the surface by taking sections of the elliptic fibration. For instance, the Brauer obstruction vanishes for the two curves defined by $r=-(s-4)(s+2) /(4(s-2))$ and $r=s(s+2) /((s-2)(3 s-2))$, yielding families of genus- 2 curves parametrized by $s$, whose Jacobians have real multiplication by $\mathcal{O}_{73}$. 


\begin{tabular}{l|c}
$(r, s)$ & Sextic polynomial $f_{6}(x)$ defining the genus-2 curve $y^{2}=f_{6}(x)$ \\
\hline$\left(-\frac{10}{3}, \frac{4}{3}\right)$ & $-4 x^{6}-12 x^{5}-23 x^{4}+4 x^{3}+31 x^{2}+57 x-18$ \\
$(-3,1)$ & $4 x^{6}-3 x^{4}-35 x^{3}+12 x+76$ \\
$(5,3)$ & $-4 x^{6}-24 x^{5}+7 x^{4}+83 x^{3}+25 x^{2}-75 x-40$ \\
$\left(\frac{9}{4}, 3\right)$ & $-15 x^{5}+73 x^{4}-41 x^{3}-158 x^{2}-12 x+36$ \\
$\left(-5, \frac{3}{2}\right)$ & $-50 x^{6}+45 x^{5}-2 x^{4}-159 x^{3}+70 x^{2}+12 x-120$ \\
$\left(\frac{5}{6},-2\right)$ & $-48 x^{6}+168 x^{5}-149 x^{4}+56 x^{3}-53 x^{2}+12 x-4$ \\
$\left(-\frac{5}{12},-1\right)$ & $195 x^{6}+82 x^{5}-75 x^{4}-186 x^{3}-233 x^{2}-96 x-87$ \\
$\left(\frac{5}{3}, \frac{1}{2}\right)$ & $-60 x^{6}-105 x^{5}+55 x^{4}+49 x^{3}+13 x^{2}+252 x+116$ \\
$\left(-\frac{2}{3},-\frac{2}{5}\right)$ & $-36 x^{6}+39 x^{5}-217 x^{4}+129 x^{3}-271 x^{2}-108 x+84$ \\
$\left(\frac{5}{4},-6\right)$ & $20 x^{6}+84 x^{5}-15 x^{4}-162 x^{3}+225 x^{2}+324 x-180$ \\
$\left(\frac{9}{5},-8\right)$ & $-204 x^{6}+348 x^{5}+27 x^{4}+34 x^{3}+3 x^{2}+108 x-36$ \\
$\left(-\frac{1}{3}, \frac{4}{5}\right)$ & $-25 x^{6}+135 x^{5}+139 x^{4}-383 x^{3}+82 x^{2}+252 x-162$ \\
$\left(\frac{9}{14},-2\right)$ & $-48 x^{6}-72 x^{5}-219 x^{4}-319 x^{3}-159 x^{2}-432 x+192$ \\
$\left(-\frac{14}{5}, 12\right)$ & $-440 x^{6}+90 x^{5}+324 x^{4}+38 x^{3}-9 x^{2}-36 x-8$ \\
$\left(-\frac{9}{4}, 1\right)$ & $-455 x^{6}-420 x^{5}+66 x^{4}-167 x^{3}-15 x^{2}-3 x-6$ \\
$\left(\frac{6}{5}, 4\right)$ & $-160 x^{6}+450 x^{5}-114 x^{4}-474 x^{3}+171 x^{2}+162 x-27$ \\
\hline
\end{tabular}

Table 22. Some rational points $(r, s)$ of small height on the surface of Theorem 37 and the corresponding genus- 2 curves.

\section{Discriminant 76}

28.1. Parametrization. Start with a $\mathrm{K} 3$ elliptic surface with fibers of type $A_{6}, A_{2}$ and $D_{7}$, and a section of height $\frac{76}{84}=\frac{19}{21}=4-1-\frac{2}{3}-\frac{10}{7}$. The Weierstrass equation of this family is

$y^{2}=x^{3}+\left(a_{0}+a_{1} t+a_{2} t^{2}+a_{3} t^{3}\right) x^{2}+t^{2}\left(b_{0}+b_{1} t+b_{2} t^{2}\right) x+t^{4}\left(c_{0}+c_{1} t+c_{2} t^{2}\right)$,

with

$$
\begin{aligned}
& c_{0}=\left(r^{2}-1\right)^{2} s^{4}(2 s-r+1)^{2}(2 s+r+1)^{2} / 16, \\
& b_{0}=\left(r^{2}-1\right) s^{2}(2 s-r+1)(2 s+r+1) / 2, \\
& a_{3}=\left(r^{2}-1\right)(s+1)^{6}, \\
& a_{1}=r^{2} s^{2}-5 s^{2}+r^{2} s-7 s+r^{2}-3, \\
& c_{2}=\left(r^{2}-1\right)^{2} r^{2} s^{6}(s+1)^{6}, \\
& a_{2}=(s+1)^{2}\left(r^{2} s^{2}+3 s^{2}-3 r^{2} s+7 s-2 r^{2}+3\right), \\
& a_{0}=1,
\end{aligned}
$$




$$
\begin{aligned}
& b_{2}=\left(r^{2}-1\right) s^{2}(s+1)^{4}\left(2 r^{2} s^{2}+6 s^{2}-2 r^{2} s+6 s+r^{4}-2 r^{2}+1\right) / 2, \\
& c_{1}=\left(r^{2}-1\right)^{2} s^{4}(s+1)^{2}(2 s-r+1)(2 s+r+1) \\
& \times\left(4 r^{2} s^{2}-12 s^{2}-8 s-r^{4}+2 r^{2}-1\right) / 16, \\
& b_{1}=-\left(r^{2}-1\right) s^{2}\left(\left(2 s^{2}+3 s+2\right) r^{4}-2\left(4 s^{4}+8 s^{3}+10 s^{2}+8 s+3\right) r^{2}\right. \\
& \left.+(2 s+1)\left(16 s^{3}+32 s^{2}+21 s+4\right)\right) / 4 .
\end{aligned}
$$

We identify the class of an $E_{7}$ fiber:

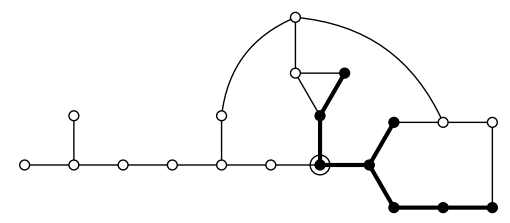

The resulting 3-neighbor step gives us an elliptic fibration with $D_{8}$ and $E_{7}$ fibers, and also a section $P$ of height $\frac{19}{2}=4+2 \cdot 4-\frac{3}{2}-1$. Next we take a 2 -neighbor step to go from $D_{8}$ to $E_{8}$, keeping the $E_{7}$ fiber intact.

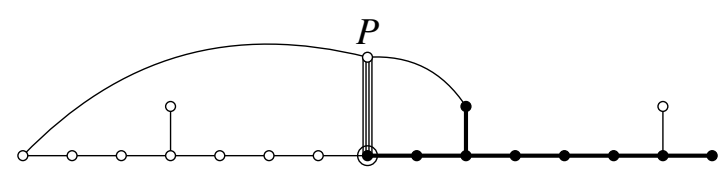

The intersection number of $F^{\prime}$ with the remaining component of the $D_{8}$ fiber is 2 , whereas $P \cdot F^{\prime}=11$. Therefore the new fibration has a section.

Now we may read out the Igusa-Clebsch invariants from the Weierstrass equation of this $E_{8} E_{7}$ fibration, and thence compute the equation of $Y_{-}(76)$ as a double cover of the $r, s$-plane, following our general method of Section 4.

Theorem 38. A birational model over $\mathbb{Q}$ for the Hilbert modular surface $Y_{-}(76)$ as a double cover of $\mathbb{P}^{2}$ is given by the equation

$z^{2}=-(r s-3 s-2)(r s+3 s+2)\left(32 s^{4}+80 s^{3}-13 r^{2} s^{2}+85 s^{2}-4 r^{2} s+32 s+4 r^{4}\right)$.

It is a surface of general type.

28.2. Analysis. The branch locus has three components; the more complicated one is where the elliptic K3 surface has an extra $\mathrm{I}_{2}$ fiber, while the two simpler components correspond to an extra $\mathrm{I}_{2}$ fiber as well as the section becoming divisible by 2 , giving a section of height $\frac{19}{84}=4-\frac{1}{2}-\frac{2}{3}-\frac{6}{7}-\frac{7}{4}$. The simpler components are easily seen to be curves of genus 0 . The last component is a curve of genus 1 ; the transformation

$$
(r, s)=\left(\frac{2 y+x+1}{x^{2}+x+2}, \frac{-2}{x^{2}+x+2}\right)
$$


converts it to Weierstrass form

$$
y^{2}+x y+y=x^{3}+x^{2}+1,
$$

which is an elliptic curve of conductor 38. It is isomorphic to $X_{0}(76) /\left\langle w_{4}, w_{19}\right\rangle$.

The Hilbert modular surface $Y_{-}(76)$ is a surface of general type. The extra involution is $\iota:(r, s) \mapsto(-r, s)$. Next, we analyze the quotient of the surface by $\iota$. This turns out to be an elliptic K3 surface, and after some Weierstrass transformations and linear shift of parameter on the base, its Weierstrass equation may be written as

$$
y^{2}=x^{3}+\left(13 t^{4}-48 t^{3}-6 t^{2}+8 t+1\right) x^{2}+64 t^{4}(2 t-1)\left(t^{3}-5 t^{2}+7 t+1\right) x .
$$

It has fibers of type $\mathrm{I}_{8}$ at $t=0, \mathrm{I}_{5}$ at $t=1, \mathrm{I}_{3}$ at $t=-\frac{1}{7}$, and $\mathrm{I}_{2}$ at $t=\frac{1}{2}$ and at the roots of $t^{3}-5 t^{2}+7 t+1$ (which generates the cubic field of discriminant $-76)$. The trivial lattice has rank 19. In addition to the obvious 2-torsion section $P_{0}=(0,0)$, we find a section $P_{1}=\left(16 t^{3}(2 t-1), 16 t^{3}(t-1)(2 t-1)(7 t+1)\right)$ of height $\frac{19}{120}$. Therefore the K3 surface is singular. These sections and the trivial lattice generate a sublattice of the Néron-Severi lattice of discriminant -76. It must be the entire Néron-Severi lattice, since otherwise, we would have either another 2-torsion section, a 4-torsion section, or a section of height $\frac{19}{480}$, none of which is possible with this configuration of reducible fibers.

The quotient of $Y_{-}$(76) by the involution $(r, s, z) \mapsto(-r, s,-z)$ is an honestly elliptic surface with $\chi=3$. Its Weierstrass equation may be written as follows:

$$
\begin{aligned}
y^{2}=x^{3}+(t-1) & \left(64 t^{5}-160 t^{4}+53 t^{3}+33 t^{2}-5 t-1\right) x^{2} \\
+ & 16(t-1) t^{4}(2 t-1)\left(t^{3}-5 t^{2}+7 t+1\right)\left(32 t^{3}-16 t^{2}+21 t-5\right) x .
\end{aligned}
$$

It has bad fibers of type $\mathrm{I}_{8}$ at $t=0, \mathrm{I}_{4}$ at $t=\infty$ and $t=\frac{1}{3}$, III at $t=1, \mathrm{I}_{3}$ at $t=-\frac{1}{7}$, and $\mathrm{I}_{2}$ at $t=\frac{1}{2}$, at the roots of $t^{3}-5 t^{2}+7 t+1$ seen above, and at the roots of $32 t^{3}-16 t^{2}+21 t-5$ (which generates the cubic field of discriminant -152 ). Hence the trivial lattice has rank 25, leaving room for Mordell-Weil rank at most 5. Counting points on the reduction modulo 11 and 23 shows that the Picard number is at most 29. On the other hand, we find three independent sections in addition to the 2-torsion section $P_{0}=(0,0)$ :

$$
\begin{aligned}
& P_{1}=\left(152(t-1) t^{4}(2 t-1), 8 \mu(t-1) t^{4}(2 t-1)(3 t-1)(4 t-3)(7 t+1)\right), \\
& P_{2}=\left(4\left(t^{3}-5 t^{2}+7 t+1\right) t^{3}, 4 t^{3}(2 t-1)(3 t-1)^{2}\left(t^{3}-5 t^{2}+7 t+1\right)\right), \\
& P_{3}=\left(-(t-1)^{3}\left(32 t^{3}-16 t^{2}+21 t-5\right), 2 v(t-1)^{2}(3 t-1)^{2}\left(32 t^{3}-16 t^{2}+21 t-5\right)\right) .
\end{aligned}
$$

Here $\mu=\sqrt{19}$ and $v=\sqrt{-1}$. These sections have heights $\frac{23}{12}, \frac{9}{8}$ and $\frac{13}{8}$ respectively, and are orthogonal with respect to the height pairing. Therefore, the Mordell-Weil rank is either 3 or 4 ; we have not been able to determine it exactly. 


\begin{tabular}{l|c}
$(r, s)$ & Sextic polynomial $f_{6}(x)$ defining the genus-2 curve $y^{2}=f_{6}(x)$ \\
\hline$\left(-\frac{4}{11},-\frac{8}{11}\right)$ & $-8 x^{6}+48 x^{5}-196 x^{4}+324 x^{3}-340 x^{2}-330 x-65$ \\
$(2,-4)$ & $375 x^{6}+300 x^{5}+230 x^{4}-224 x^{3}-76 x^{2}-48 x+72$ \\
$\left(\frac{7}{3},-\frac{1}{3}\right)$ & $-80 x^{6}-120 x^{5}-109 x^{4}-348 x^{3}-469 x^{2}-120 x+80$ \\
$(-2,-4)$ & $-225 x^{6}+600 x^{5}+650 x^{4}+400 x^{3}+20 x^{2}-8$ \\
$\left(\frac{13}{23},-\frac{19}{23}\right)$ & $-228 x^{6}+684 x^{5}-1029 x^{4}-432 x^{3}+525 x^{2}+150 x+10$ \\
$\left(-\frac{7}{3},-\frac{1}{3}\right)$ & $100 x^{6}-220 x^{5}+621 x^{4}-528 x^{3}+1699 x^{2}-234 x+1478$ \\
$\left(\frac{19}{33},-\frac{9}{11}\right)$ & $256 x^{6}-1056 x^{5}-2335 x^{4}+1480 x^{3}+1715 x^{2}-1386 x+126$ \\
$\left(\frac{19}{11}, \frac{1}{11}\right)$ & $-2232 x^{6}-2016 x^{5}+2581 x^{4}+2802 x^{3}-983 x^{2}-660 x-180$ \\
$\left(\frac{47}{37},-\frac{43}{37}\right)$ & $3100 x^{6}-540 x^{5}-271 x^{4}-1742 x^{3}+161 x^{2}+84 x+252$ \\
$\left(\frac{43}{27}, \frac{29}{27}\right)$ & $-592 x^{6}+372 x^{5}+1003 x^{4}+1328 x^{3}-1406 x^{2}-132 x-3709$ \\
$\left(-\frac{19}{33},-\frac{9}{11}\right)$ & $-4404 x^{6}-540 x^{5}-1697 x^{4}-980 x^{3}-257 x^{2}-240 x-64$ \\
$\left(\frac{22}{17},-\frac{20}{17}\right)$ & $600 x^{6}-3360 x^{5}+3604 x^{4}+2256 x^{3}+4546 x^{2}+1440 x+775$ \\
$\left(-\frac{22}{13},-\frac{20}{13}\right)$ & $-367 x^{6}-618 x^{5}-1539 x^{4}+316 x^{3}+1839 x^{2}+4662 x+3507$ \\
$\left(-\frac{1}{23},-\frac{31}{46}\right)$ & $-2245 x^{6}-137 x^{5}-5393 x^{4}-1675 x^{3}-3618 x^{2}-1728 x-675$ \\
$\left(\frac{22}{13},-\frac{20}{13}\right)$ & $1425 x^{6}-2610 x^{5}+6333 x^{4}-4948 x^{3}+8271 x^{2}-4242 x+5971$ \\
$\left(-\frac{1}{23},-\frac{17}{23}\right)$ & $24 x^{6}+552 x^{5}+2075 x^{4}-1970 x^{3}-9925 x^{2}+9072 x+1216$ \\
\hline
\end{tabular}

Table 23. Some rational points $(r, s)$ of small height on the surface of Theorem 38 and the corresponding genus- 2 curves.

28.3. Examples. Table 23 lists some points of small height and their genus- 2 curves.

We now describe some curves on $Y_{-}(76)$, which are useful in producing rational points.

The specialization $s=-\frac{2}{3}$ gives a genus- 1 curve $y^{2}=-81 r^{4}+63 r^{2}+19$. It has rational points, such as $(r, y)=(1,1)$. It is thus an elliptic curve; we find that it has conductor 760 and Mordell-Weil group $(\mathbb{Z} / 2 \mathbb{Z}) \oplus \mathbb{Z}$. The specialization $s=-\frac{8}{7}$ gives a rational curve, which we can parametrize as $r=-5\left(m^{2}-1\right) /\left(4\left(m^{2}+1\right)\right)$.

The sections $P_{1}, P_{1}+P_{0}, 2 P_{1}+P_{0}$ and $3 P_{1}+P_{0}$ of the $\mathrm{K} 3$ quotient give the following genus-1 curves, which all have rational points.

Equation

$$
\begin{aligned}
& r^{2}=-\left(8 s^{3}+28 s^{2}+27 s+8\right) / s \\
& r^{2}=-\left(s^{3}-s^{2}-11 s-8\right) / s \\
& r^{2}=-\left(8 s^{4}+4 s^{3}-33 s^{2}-44 s-16\right) /(s+2)^{2} \\
& r^{2}=-\left(s^{4}+s^{3}-3 s^{2}-s+1\right)
\end{aligned}
$$

conductor Mordell-Weil group

$\begin{array}{cc}2 \cdot 29 & \mathbb{Z} \\ 2^{4} 11 \cdot 191 & \mathbb{Z}^{2} \\ 3^{5} 19 & \mathbb{Z}^{2} \\ 2^{2} 5 \cdot 29 & (\mathbb{Z} / 2 \mathbb{Z}) \oplus \mathbb{Z}\end{array}$

The section $2 P_{1}$ gives a genus- 0 curve $r^{2}+s^{2}+6 s+4=0$, which we can parametrize as 


$$
(r, s)=\left(-\frac{m^{2}+4 m-1}{m^{2}+1},-\frac{m^{2}+2 m+5}{m^{2}+1}\right) .
$$

The Brauer obstruction always vanishes on this locus, giving us a 1-parameter family of genus- 2 curves whose Jacobians have real multiplication by $\mathcal{O}_{76}$.

\section{Discriminant 77}

29.1. Parametrization. We start with a family of $K 3$ surfaces with fibers of type $A_{1}, A_{3}, A_{5}$ and $D_{5}$, a 2-torsion section $T$, and two orthogonal sections $P, Q$ of height $\frac{11}{12}=4-0-\frac{2 \cdot 2}{4}-\frac{1 \cdot 5}{6}-\left(1+\frac{1}{4}\right)$ and $\frac{7}{4}=4-0-\frac{1 \cdot 3}{4}-\frac{3 \cdot 3}{6}-0$. The orthogonality comes from $0=2-0-\frac{1 \cdot 2}{4}-\frac{1 \cdot 3}{6}-0-1$, where the last term comes from the intersection number $(P) \cdot(Q)$ on the surface. We can write the Weierstrass equation of this family as

$$
\begin{aligned}
y^{2}=x^{3}+\left((r s-1)^{2}-\right. & \left(s^{2}-1\right)\left(r s-4 r^{2}-1\right) t \\
& \left.+r\left(s^{2}-1\right)\left(r s^{2}+8 s-57 r\right) t^{2} / 4+8 r^{2}\left(s^{2}-1\right) t^{3}\right) x^{2} \\
& +r^{2}\left(s^{2}-1\right)^{2} t^{3}(t-1)^{2}\left(16 r^{2} t+(r s-1)^{2}-(s-5 r)^{2}\right) x .
\end{aligned}
$$

We go to $E_{8} E_{7}$ form in three steps, via $D_{8} E_{6}$ and $E_{8} E_{6}$.

First, we identify a $D_{8}$ fiber $F^{\prime}$ in the figure below (we omit drawing the node representing $Q$ and the edges connecting it to the rest of the diagram, as it would clutter up the picture).

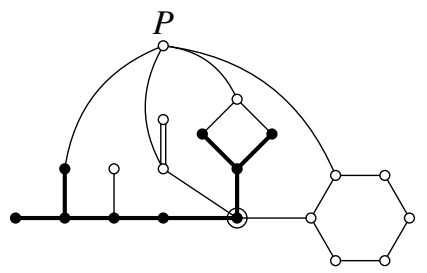

The section $P$ intersects $F^{\prime}$ once, and so the new fibration has a section. It has $D_{8}$ and $E_{6}$ fibers and rank 2 .

Next, we identify the class of an $E_{8}$ fiber below, and go to an elliptic fibration with $E_{8}$ and $E_{6}$ fibers, by a 2-neighbor step.

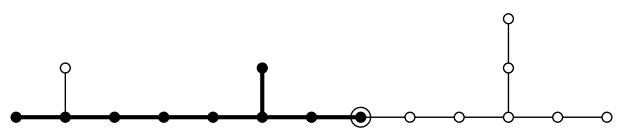

The resulting elliptic fibration has Mordell-Weil lattice of rank 2 and discriminant $\frac{77}{3}$. We can relatively easily describe a section $P^{\prime \prime}$ of height $\frac{8}{3}$, which intersects a non-identity component of the $E_{6}$ fiber. 
Now the $E_{7}$ fiber $F^{\prime \prime \prime}$ drawn below defines an elliptic fibration with a section and with $E_{8}$ and $E_{7}$ fibers.

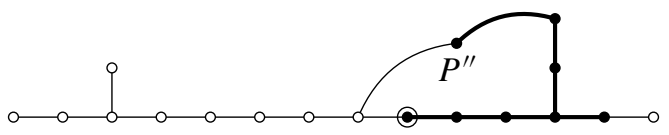

We now read out the Igusa-Clebsch invariants and proceed as in Section 4 to compute the equation of $Y_{-}(77)$ as a double cover of the Humbert surface $\mathcal{H}_{77}$.

Theorem 39. A birational model over $\mathbb{Q}$ for the Hilbert modular surface $Y_{-}(77)$ as a double cover of $\mathbb{P}^{2}$ is given by the equation

$$
\begin{aligned}
z^{2}=r^{2}(r-1)^{2}(r+1)^{2} s^{6}+2 r(r-1)(r+1)\left(21 r^{2}-13\right) s^{5} \\
-\left(14 r^{6}-433 r^{4}+328 r^{2}+27\right) s^{4}-4 r\left(281 r^{4}-522 r^{2}-79\right) s^{3} \\
-\left(343 r^{6}+6268 r^{4}+1763 r^{2}-54\right) s^{2}+2 r\left(3997 r^{4}+2446 r^{2}-171\right) s \\
-\left(1372 r^{6}+4531 r^{4}-362 r^{2}+27\right) .
\end{aligned}
$$

It is a surface of general type.

\begin{tabular}{|c|c|}
\hline$(r, s)$ & Sextic polynomial $f_{6}(x)$ defining the genus- 2 curve $y^{2}=f_{6}(x)$ \\
\hline & $1581 x^{6}-25965 x^{5}+128199 x^{4}-124655 x^{3}-282240 x^{2}-92400 x-9478$ \\
\hline & $\begin{array}{rl}926711 x^{6}-351913 x^{5}-2531791 x^{4}+699677 x^{3}+2 & 216646 x^{2} \\
& -359536 x-579882\end{array}$ \\
\hline$\left(\frac{1}{2}, \frac{23}{5}\right)$ & $\begin{array}{r}2038350 x^{6}+1601640 x^{5}-6288456 x^{4}+116705 x^{3}-5729115 x^{2} \\
-8845053 x-2931103\end{array}$ \\
\hline$\left(-\frac{13}{2}, 6\right)$ & $\begin{array}{r}10433826 x^{6}-16243110 x^{5}+25749477 x^{4}-25899800 x^{3}+5297523 x^{2} \\
-6454140 x-9577876\end{array}$ \\
\hline$\left(\frac{13}{2},-6\right)$ & $\begin{array}{r}-749865564 x^{6}+4317895148 x^{5}+1178682897 x^{4}-6739621816 x^{3} \\
-800208729 x^{2}+2973824982 x+128797182\end{array}$ \\
\hline$\left(-\frac{33}{65},-\frac{239}{63}\right)$ & $\begin{array}{r}-5738303278944 x^{6}+6551435295576 x^{5} \\
+28045528925148 x^{4}-5100723398753 x^{3}-23656013198837 x^{2} \\
+ \\
+3333165270637 x+3904336668117\end{array}$ \\
\hline
\end{tabular}

29.2. Analysis. It is a surface of general type. It has an extra involution $(r, s) \mapsto$ $(-r,-s)$. The branch locus is a curve of genus 2 . The change of coordinates

$$
(r, s)=\left(\frac{4 x y-5 x^{4}-22 x^{2}-21}{x\left(x^{2}+7\right)^{2}}, \frac{-\left(2 x^{2} y+2 y-x^{5}-6 x^{3}-9 x\right)}{\left(x^{2}-1\right)^{2}}\right)
$$

Table 24. Some rational points $(r, s)$ of small height on the surface of Theorem 39 and the corresponding genus- 2 curves. 
converts it to Weierstrass form

$$
y^{2}=x^{6}+5 x^{4}+3 x^{2}+7
$$

It is isomorphic to the quotient of $X_{0}(77)$ by the Atkin-Lehner involution $w_{77}$.

29.3. Examples. Table 24 on the previous page lists some points of small height and their genus- 2 curves.

\section{Discriminant 85}

30.1. Parametrization. We start with a $\mathrm{K} 3$ elliptic surface with fibers of type $E_{6}, D_{5}$ and $A_{4}$, with a section of height $\frac{85}{60}=\frac{17}{12}=4-\frac{4}{3}-\frac{5}{4}$.

The Weierstrass equation is

$$
y^{2}=x^{3}+t\left(a_{0}+a_{1} t\right) x^{2}+2 t^{2}(t-1)\left(b_{0}+b_{1} t\right) x+t^{3}(t-1)^{4}\left(c_{0}+c_{1} t\right),
$$

with

$$
\begin{aligned}
& a_{0}=-4\left(e^{2}-1\right)\left(3 e f^{2}+2 e^{2} f+2 f-2 e^{2}+e+2\right), \\
& c_{0}=-64 e^{2}\left(e^{2}-1\right)^{3}\left(f^{2}-1\right)^{2}(f+2 e+1)(e f-e+2), \\
& a_{1}=4\left(e^{2}-1\right)^{2} f^{2} \\
& b_{0}=8 e\left(e^{2}-1\right)^{2}\left(f^{2}-1\right)\left(3 e f^{2}+4 e^{2} f+4 f-4 e^{2}+5 e+4\right), \\
& b_{1}=-8 f\left(e^{2}-1\right)^{2}\left(f^{2}-1\right)\left(e^{4} f+e^{3} f-e f+f-e^{4}+e^{3}+e+1\right), \\
& c_{1}=16\left(e^{2}-1\right)^{2}\left(f^{2}-1\right)^{2}\left(e^{4} f+e^{3} f-e f+f-e^{4}+e^{3}+e+1\right)^{2} .
\end{aligned}
$$

We identify the class of a $D_{8}$ fiber below, and move to the new elliptic fibration via a 2-neighbor step.

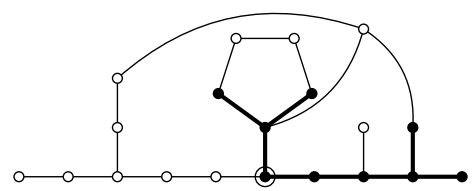

The new elliptic fibration has reducible fibers of type $D_{8}$ and $E_{6}$, and MordellWeil rank 2. Next, we move to an elliptic fibration with $E_{8}$ and $E_{6}$ fibers by another 2-neighbor step, using the $E_{8}$ fiber shown below.

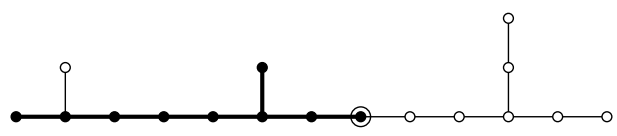

The new elliptic fibration has $E_{8}$ and $E_{6}$ fibers. We can find an explicit section $P$ of the $E_{8} E_{6}$ fibration which intersects a non-identity component of the $E_{6}$ fiber and does not intersect the zero section. 
Let $F$ be an $E_{7}$ fiber enclosed by the box in the picture below. We move to it by a 2-neighbor step, to recover an elliptic fibration with $E_{8}$ and $E_{7}$ fibers.

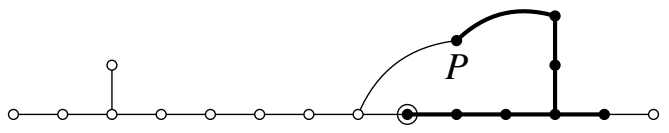

We may now read out the Igusa-Clebsch invariants, and work out the equation of $Y_{-}(85)$ as a double cover of $\mathbb{P}_{e, f}^{2}$.

Theorem 40. A birational model over $\mathbb{Q}$ for the Hilbert modular surface $Y_{-}(85)$ as a double cover of $\mathbb{P}_{e, f}^{2}$ is given by the equation

$$
\begin{aligned}
z^{2}=-\left(e^{2}-\right. & e-1)^{2}\left(8 e^{4}+11 e^{2}+8\right) f^{4} \\
& +4\left(e^{4}-1\right)\left(18 e^{4}-11 e^{3}+27 e^{2}+11 e+18\right) f^{3} \\
& -2\left(82 e^{8}-118 e^{7}-9 e^{6}-60 e^{5}-173 e^{4}+60 e^{3}-9 e^{2}+118 e+82\right) f^{2} \\
& +4\left(e^{4}-1\right)\left(4 e^{2}-5 e-4\right)\left(9 e^{2}-13 e-9\right) f \\
& -\left(2 e^{2}-e-2\right)^{2}\left(11 e^{4}-34 e^{3}+5 e^{2}+34 e+11\right) .
\end{aligned}
$$

It is an honestly elliptic surface, of arithmetic genus 3 and Picard number 37 or 38.

30.2. Analysis. It is an honestly elliptic surface, with extra involution $\iota:(e, f, z) \mapsto$ $\left(-1 / e,-f, z / e^{4}\right)$.

The branch locus is a hyperelliptic curve of genus 3 . The change of coordinates

$(e, f)=\left(\frac{y+x^{4}+x^{3}-x+1}{2 x^{2}+2 x-1}, \frac{\left(3 x^{4}+x^{3}-3 x^{2}-x+3\right) y-\left(x^{2}-1\right)\left(x^{2}+1\right)^{3}}{\left(x^{2}+x-1\right)^{2}\left(2 x^{4}-x^{2}+2\right)}\right)$

converts it to Weierstrass form:

$$
y^{2}=x^{8}+2 x^{7}-x^{6}-8 x^{5}+x^{4}+8 x^{3}-x^{2}-2 x+1 .
$$

It is isomorphic to the quotient of $X_{0}(85)$ by the Atkin-Lehner involution $w_{85}$.

The equation of $Y_{-}(85)$ describes it as an elliptic surface over $\mathbb{P}_{e}^{1}$. So far, we are unable to find a section.

The Jacobian of this genus-1 curve over $\mathbb{Q}(e)$ can be written (after some Weierstrass transformations) as

$$
\begin{aligned}
y^{2}=x^{3}+\left(e^{8}-\right. & \left.10 e^{7}+3 e^{6}+84 e^{5}+85 e^{4}-84 e^{3}+3 e^{2}+10 e+1\right) x^{2} \\
- & 8 e^{5}\left(23 e^{6}-133 e^{5}-420 e^{4}-64 e^{3}+420 e^{2}-133 e-23\right) x \\
- & 16 e^{9}\left(108 e^{6}-637 e^{5}-1944 e^{4}-26 e^{3}+1944 e^{2}-637 e-108\right) .
\end{aligned}
$$

It has $\chi=4$. There are bad fibers of type $\mathrm{I}_{9}$ at $e=0$ and $e=\infty, \mathrm{I}_{3}$ at $e= \pm 1, \mathrm{I}_{2}$ at the roots of $e=4 \pm \sqrt{17}$, and $\mathrm{I}_{3}$ at the roots of $3 e^{6}-16 e^{5}-54 e^{4}+5 e^{3}+54 e^{2}-16 e-3$ 
(which generates the compositum of $\mathbb{Q}(\sqrt{85})$ and the cubic field of discriminant -3 . $5 \cdot 17)$. The trivial lattice has rank 36 , leaving room for at most four independent sections. So far, we can only say from the ensuing analysis that the rank is either 1 or 2 .

Next, we analyze the quotient of this surface by the involution $\iota$. In terms of $g=e-1 / e$ and $h=f /(e+1 / e)$ (which are invariant under $\iota$ ), its equation is

$$
\begin{aligned}
z^{2}=-(g-1)^{2}( & \left.g^{2}+4\right)^{2}\left(8 g^{2}+27\right) h^{4}+4 g\left(g^{2}+4\right)^{2}\left(18 g^{2}-11 g+63\right) h^{3} \\
& -2\left(g^{2}+4\right)\left(82 g^{4}-118 g^{3}+319 g^{2}-414 g-27\right) h^{2} \\
+ & 4 g(4 g-5)(9 g-13)\left(g^{2}+4\right) h-(2 g-1)^{2}\left(11 g^{2}-34 g+27\right) .
\end{aligned}
$$

This is also an honestly elliptic surface, this time with $\chi=3$. Its Jacobian is

$$
\begin{aligned}
& y^{2}=x^{3}+\left(g^{2}+4\right)\left(g^{4}-10 g^{3}+7 g^{2}+66 g-27\right) x^{2} \\
& +8 g(g-8)\left(g^{2}+4\right)^{2}\left(g^{3}-12 g^{2}-12 g+27\right) x+16 g^{2}(g-8)^{2}\left(g^{2}+4\right)^{3}\left(g^{2}-14 g-27\right) .
\end{aligned}
$$

It has bad fibers of type $\mathrm{I}_{9}$ at $g=\infty, \mathrm{I}_{3}$ at $g=0, \mathrm{I}_{2}$ at $g=8, \mathrm{I}_{0}^{*}$ at $g= \pm 2 \sqrt{-1}$, and $\mathrm{I}_{3}$ at the roots of $3 g^{3}-16 g^{2}-45 g-27$ (which generates the cubic field of discriminant -255). The trivial lattice has rank 27, leaving room for Mordell-Weil rank at most 3. Counting points modulo 11 and 19 shows that the Picard number is at most 29. On the other hand, we are able to find the non-torsion section

$$
\begin{aligned}
P_{1}=\left(-4(g-8)\left(g^{2}+4\right)\left(9 g^{3}+3 g^{2}+g+72\right) / 85,\right. \\
\\
\left.\quad(g-8)(21 g-4)\left(g^{2}+4\right)^{2}\left(3 g^{3}-16 g^{2}-45 g-27\right) / 85^{3 / 2}\right)
\end{aligned}
$$

of height $\frac{3}{2}$. Therefore, the Mordell-Weil rank is either 1 or 2 .

Next, we consider the quadratic twist of the quotient elliptic surface, which is obtained by simply removing the factors of $\left(g^{2}+4\right)$ in the Weierstrass equation above (recalling that $g^{2}+4=(e+1 / e)^{2}$ ). We get a K3 surface with a genus-1 fibration, whose Jacobian has Weierstrass equation

$$
\begin{aligned}
y^{2}=x^{3}+ & \left(g^{4}-10 g^{3}+7 g^{2}+66 g-27\right) x^{2} \\
& +8 g(g-8)\left(g^{3}-12 g^{2}-12 g+27\right) x+16 g^{2}(g-8)^{2}\left(g^{2}-14 g-27\right) .
\end{aligned}
$$

It has reducible fibers of type $\mathrm{I}_{9}$ at $g=\infty, \mathrm{I}_{2}$ at $g=8$, and $\mathrm{I}_{3}$ at $g=0$ and at the roots of $3 g^{3}-16 g^{2}-45 g-27$. Therefore the trivial lattice has rank 19, and the Mordell-Weil rank can be 0 or 1 . We find a 3-torsion section

$$
P_{0}=\left(8 g+36,4\left(3 g^{3}-16 g^{2}-45 g-27\right)\right) .
$$

Counting points modulo 7 and 19 shows that the Picard number is exactly 19 . The 3 -torsion section and trivial lattice span a sublattice of discriminant $162=2 \cdot 3^{4}$ of the Néron-Severi lattice of discriminant. It is easy to check that this sublattice is 3-saturated, and therefore must form the entire Néron-Severi lattice. 


\begin{tabular}{l|c}
$(e, f)$ & Sextic polynomial $f_{6}(x)$ defining the genus-2 curve $y^{2}=f_{6}(x)$ \\
\hline$\left(\frac{1}{2},-\frac{29}{15}\right)$ & $576 x^{6}+432 x^{5}+927 x^{4}+81 x^{3}+171 x^{2}-72 x-208$ \\
$\left(\frac{4}{3}, \frac{1}{7}\right)$ & $-1344 x^{6}-672 x^{5}-2233 x^{4}-3026 x^{3}-997 x^{2}-2196 x-548$ \\
$\left(\frac{7}{2}, \frac{8}{17}\right)$ & $-1566 x^{6}-7704 x^{5}-4056 x^{4}-8581 x^{3}-5841 x^{2}-2055 x-2395$ \\
$\left(-2, \frac{29}{15}\right)$ & $-3500 x^{6}-2100 x^{5}+11205 x^{4}+2422 x^{3}-11295 x^{2}+1080 x+2160$ \\
$\left(-2, \frac{23}{21}\right)$ & $-316 x^{6}+3048 x^{5}+14649 x^{4}+10547 x^{3}-13509 x^{2}-1296 x+1728$ \\
$\left(-\frac{6}{7},-13\right)$ & $-5028 x^{6}-10620 x^{5}-2605 x^{4}-16750 x^{3}+5255 x^{2}-6600 x+2832$ \\
$\left(-\frac{1}{2},-7\right)$ & $8964 x^{6}-3132 x^{5}+18927 x^{4}+6286 x^{3}+6655 x^{2}+11300 x-500$ \\
$\left(\frac{2}{5},-\frac{7}{3}\right)$ & $21006 x^{6}-45414 x^{5}+16263 x^{4}-20048 x^{3}-7227 x^{2}+960 x-3200$ \\
$\left(-\frac{3}{4},-\frac{3}{5}\right)$ & $5500 x^{6}+30300 x^{5}+19835 x^{4}+20174 x^{3}-46885 x^{2}+2340 x-380$ \\
$\left(\frac{5}{2}, \frac{7}{3}\right)$ & $-12852 x^{6}-15876 x^{5}+40383 x^{4}+49976 x^{3}-30231 x^{2}-43650 x+2250$ \\
$\left(\frac{11}{20},-\frac{71}{49}\right)$ & $-66020 x^{6}+43980 x^{5}+10001 x^{4}+1154 x^{3}-5899 x^{2}-1464 x+1096$ \\
$\left(-\frac{2}{5},-\frac{7}{3}\right)$ & $-72620 x^{6}+37884 x^{5}-12135 x^{4}+29302 x^{3}-4107 x^{2}+1848 x-2672$ \\
$\left(-\frac{5}{2}, \frac{7}{3}\right)$ & $20 x^{6}+180 x^{5}-3879 x^{4}-34668 x^{3}+44937 x^{2}+62856 x-73296$ \\
$\left(\frac{7}{6}, 13\right)$ & $-5442 x^{6}+3630 x^{5}-7079 x^{4}-93460 x^{3}+35059 x^{2}-420 x+9212$ \\
$\left(-4, \frac{11}{9}\right)$ & $-16964 x^{6}-33804 x^{5}+53325 x^{4}+100170 x^{3}-35163 x^{2}-81540 x-1116$ \\
$\left(\frac{11}{2}, \frac{9}{13}\right)$ & $-102046 x^{6}+130482 x^{5}+61857 x^{4}+9504 x^{3}-74697 x^{2}-38412 x-14036$ \\
\hline
\end{tabular}

Table 25. Some rational points $(e, f)$ of small height on the surface of Theorem 40 and the corresponding genus- 2 curves.

30.3. Examples. Table 25 lists some points of small height and their genus-2 curves.

\section{Discriminant 88}

31.1. Parametrization. We start with an elliptic $\mathrm{K} 3$ surface with fibers of type $A_{9}$, $D_{4}$ and $A_{2}$, and a section of height $\frac{11}{15}=4-\frac{2}{3}-1-\frac{16}{10}$. The Weierstrass equation for this family is

$$
\begin{aligned}
y^{2}=x^{3}+\left(a_{0}+\right. & \left.a_{1} t+a_{2} t^{2}+a_{3} t^{3}\right) x^{2} \\
& +2 t^{2}(\lambda t-\mu)\left(b_{0}+b_{1} t+b_{2} t^{2}+b_{3} t^{3}\right) x+t^{4}(\lambda t-\mu)^{2}\left(c_{0}+c_{1} t\right)^{2},
\end{aligned}
$$

with

$$
\begin{aligned}
& a_{0}=1, \quad \mu=r s+2 s+1, \quad \lambda=s(r+2)^{2}(2 s+1)^{2}, \\
& c_{0}=-2, \quad b_{0}=2, \quad a_{1}=-8 s(r s+2 r+1), \\
& c_{1}=8(r+2) s^{2}+8(r+1) s+r^{2}, \\
& b_{3}=2 r(r+2) s(2 s+1)\left(8 s+r^{2}\right)\left(8 s^{2}+r\right), \\
& b_{2}=32 r(r+2) s^{4}+32\left(3 r^{2}+4 r+2\right) s^{3}-4\left(r^{3}-20 r^{2}-24 r-8\right) s^{2}+4(r-1) r^{2} s,
\end{aligned}
$$




$$
\begin{aligned}
& b_{1}=-16(r+1) s^{2}-8(3 r+2) s-r^{2}, \\
& \begin{aligned}
& a_{3}=4 r s\left(64 r s^{4}+16\left(r^{3}+3 r^{2}+\right.\right.12 r+4) s^{3} \\
&\left.+2 r\left(r^{2}+4\right) s^{2}+r\left(r^{3}+12 r^{2}+12 r+16\right) s+r^{3}\right), \\
& a_{2}=4 s\left(4 r^{2} s^{3}+8 r(2 r-1) s^{2}-4\left(r^{3}-4 r^{2}-4 r-1\right) s-r^{2}(r+4)\right) .
\end{aligned}
\end{aligned}
$$

First we identify an $E_{7}$ fiber, and make a 3-neighbor move to it.

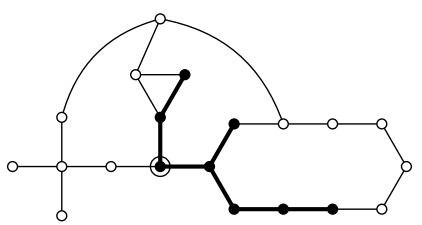

This gives us an elliptic fibration with $E_{7}, D_{5}$ and $A_{3}$ fibers, and a section of height $\frac{88}{32}=\frac{11}{4}=4-\frac{5}{4}$. Then we can identify a $D_{8}$ fiber $F^{\prime}$ below, and move to the associated genus 1 fibration by a 2-neighbor step.

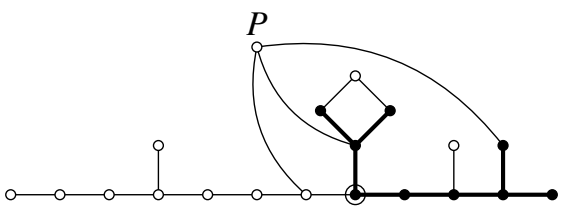

To see that the genus- 1 fibration defined by this fiber $F^{\prime}$ has a section, note that $P \cdot F^{\prime}=3$, while $F^{\prime}$ intersects the near leaf of the $D_{5}$ fiber with multiplicity 2 . Therefore we may replace the genus-1 fibration by its Jacobian.

Finally, we go by another 2-neighbor move to a fibration with $E_{8}$ and $E_{7}$ fibers. We identify the class of an $E_{8}$ fiber $F^{\prime \prime}$ below. The Mordell-Weil group is generated by a section $P^{\prime}$ of height $88 /(2 \cdot 4)=11=4+2 \cdot 4-1$, so the section must intersect the zero section with multiplicity 4 , and it must intersect the near leaf of the $D_{8}$ fiber. Therefore $P^{\prime} \cdot F^{\prime \prime}=2 \cdot 4+3=11$, whereas the omitted far leaf of the $D_{8}$ fiber intersects $F^{\prime \prime}$ with multiplicity 2 . So the new fibration has a section.

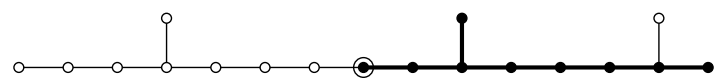

We may now read out the Igusa-Clebsch invariants and compute the equation of the branch locus for $Y_{-}(88) \mapsto \mathbb{P}_{r, s}^{2}$.

Theorem 41. A birational model over $\mathbb{Q}$ for the Hilbert modular surface $Y_{-}(88)$ as a double cover of $\mathbb{P}_{r, s}^{2}$ is given by the equation

$$
\begin{aligned}
& z^{2}=\left(8 r s^{2}+16 s^{2}+8 s+r^{2}\right)\left(8 r^{3} s^{4}+16 r^{2} s^{4}+96 r^{3} s^{3}+472 r^{2} s^{3}+544 r s^{3}\right. \\
& \left.-27 r^{4} s^{2}-120 r^{3} s^{2}+64 r^{2} s^{2}+472 r s^{2}+16 s^{2}-46 r^{3} s-120 r^{2} s+96 r s+8 s-27 r^{2}\right) .
\end{aligned}
$$


It is a surface of general type.

31.2. Analysis. The branch locus has two components. Both correspond to elliptic $\mathrm{K} 3$ surfaces with an extra $\mathrm{I}_{2}$ fiber, and the simpler component to having a 2-torsion section in addition. The simpler component of the branch locus has genus 1 ; the change of coordinates $r=2 y / x^{2}, s=-1 /(2 x)$ converts it to Weierstrass form

$$
y^{2}+y=x^{3}-x^{2}
$$

which is an elliptic curve of conductor 11 (isomorphic to $X_{1}(11)$ ).

The other component has genus 2 . The transformation

$$
\begin{aligned}
(r, s)=\left(\frac{-(x-1) y+(x+1)\left(x^{3}-3 x^{2}-3 x-1\right)}{3 x^{2}+2 x+1},\right. & \left.\frac{(3 x+1) y-(x+1)\left(3 x^{3}+3 x^{2}+3 x+1\right)}{4 x\left(x^{2}-2 x-1\right)}\right)
\end{aligned}
$$

converts it to Weierstrass form

$$
y^{2}=x^{6}-2 x^{5}+11 x^{4}+20 x^{3}+15 x^{2}+6 x+1 .
$$

The Hilbert modular surface $Y_{-}(88)$ is a surface of general type. We now analyze its quotient by the involution $\iota:(r, s, z) \mapsto\left(1 / s, 1 / r, z /(r s)^{3}\right)$. Writing $h=-(s+1 / r), m=(s-1 / r)^{2}$, we find the equation

$$
\begin{aligned}
z^{2}=\left(h^{4}-2 h^{3}-2 m h^{2}+2 m h+m^{2}+1\right)\left(9 h^{6}-30 h^{5}-26 m h^{4}+16 h^{4}+58 m h^{3}\right. \\
\left.+30 h^{3}+25 m^{2} h^{2}-8 m h^{2}-25 h^{2}-28 m^{2} h-30 m h-8 m^{3}-8 m^{2}-2 m\right) .
\end{aligned}
$$

The invertible transformation $m=2+1 / t+8 /(n t)+4 /(n t)^{2}, h=-1-2 /(n t)$ makes this a quartic in $n$,

$z^{2}=(4 t-n-4)\left(t(2 t+1)\left(6 t^{2}-13 t+8\right) n^{3}+4 t(2 t-3)^{2} n^{2}-4(2 t-1)^{2} n+16(t-1)\right)$,

with an obvious section $n=4 t-4$. Converting to the Jacobian, we get an elliptic K3 surface with the following equation (after some Weierstrass transformations and a change of parameter $t \mapsto 1-t$ on the base):

$$
\begin{aligned}
y^{2}=x^{3}+ & \left(28 t^{4}-24 t^{3}-8 t^{2}+4 t+1\right) x^{2} \\
- & 16 t^{3}(t-1)^{2}\left(t^{3}-10 t^{2}+4 t+1\right) x+64 t^{6}(t-1)^{4}\left(29 t^{2}-10 t-3\right) .
\end{aligned}
$$

This has bad fibers of type $\mathrm{I}_{6}$ at $t=0, \mathrm{I}_{5}$ at $t=1, \mathrm{I}_{2}$ at $t=\frac{1}{2}$ and $t=-\frac{1}{6}$, and $\mathrm{I}_{3}$ at $t=\frac{1}{4} \pm \frac{\sqrt{33}}{12}$. The trivial lattice has rank 17. We find the independent sections

$$
\begin{aligned}
& P_{1}=\left(-4 t(t-1)\left(7 t^{2}-2 t-1\right), 4 t(t-1)(t+1)(2 t-1)\left(6 t^{2}-3 t-1\right)\right), \\
& P_{2}=\left(4 t(t-1)^{2}(5 t+1), 4 t(t-1)^{2}(6 t+1)\left(6 t^{2}-3 t-1\right)\right),
\end{aligned}
$$




$$
P_{3}=\left(4 t^{3}(6-13 t), 12 \sqrt{-3} t^{4}(2 t-1)(6 t+1)\right),
$$

with height matrix

$$
\left(\begin{array}{ccc}
\frac{8}{15} & \frac{1}{10} & 0 \\
\frac{1}{10} & \frac{2}{15} & 0 \\
0 & 0 & \frac{3}{2}
\end{array}\right) .
$$

Therefore the K3 surface is singular, and an easy argument shows that these sections and the trivial lattice must span the Néron-Severi lattice, which therefore has rank 20 and discriminant -99 .

31.3. Examples. Table 26 lists some points of small height and their genus- 2 curves.

We describe some curves on the surface which are a source of rational points (some more may be produced by applying the involution $\iota$ ). The specialization

\begin{tabular}{l|c}
$(r, s)$ & Sextic polynomial $f_{6}(x)$ defining the genus-2 curve $y^{2}=f_{6}(x)$ \\
\hline$\left(-\frac{2}{3},-\frac{7}{10}\right)$ & $25 x^{6}+120 x^{5}-291 x^{4}-1292 x^{3}+987 x^{2}+588 x-497$ \\
$\left(-4, \frac{3}{2}\right)$ & $486 x^{6}-810 x^{5}+1323 x^{4}-800 x^{3}+585 x^{2}-48 x+64$ \\
$\left(-\frac{10}{7},-\frac{3}{2}\right)$ & $515 x^{6}+1314 x^{5}-3120 x^{4}-1332 x^{3}+2292 x^{2}+720 x-200$ \\
$\left(-\frac{4}{7},-\frac{3}{10}\right)$ & $20 x^{6}+180 x^{5}-159 x^{4}-3276 x^{3}+249 x^{2}+1980 x-1100$ \\
$\left(\frac{2}{3},-\frac{1}{4}\right)$ & $4608 x^{6}+6048 x^{5}+3771 x^{4}-1026 x^{3}+351 x^{2}-36 x+4$ \\
$\left(-\frac{20}{7},-\frac{5}{6}\right)$ & $356 x^{6}-4980 x^{5}+6373 x^{4}+2580 x^{3}-4409 x^{2}-4170 x-790$ \\
$\left(\frac{8}{21},-\frac{2}{5}\right)$ & $1664 x^{6}+624 x^{5}+3747 x^{4}-5222 x^{3}+5511 x^{2}-1140 x+15020$ \\
$\left(-\frac{6}{5},-\frac{7}{20}\right)$ & $6260 x^{6}-21060 x^{5}+7009 x^{4}-1254 x^{3}-239 x^{2}-540 x-100$ \\
$\left(-\frac{10}{3},-\frac{7}{4}\right)$ & $2 x^{6}+54 x^{5}+45 x^{4}+1080 x^{3}-2961 x^{2}-44352$ \\
$\left(-\frac{4}{3},-\frac{13}{6}\right)$ & $-14388 x^{6}-86076 x^{5}-115441 x^{4}+70272 x^{3}+86417 x^{2}-10794 x+314$ \\
$\left(-\frac{22}{21},-\frac{13}{70}\right)$ & $7865 x^{6}-9750 x^{5}+62049 x^{4}-2788 x^{3}+162759 x^{2}-4350 x+119375$ \\
$\left(-\frac{5}{2}, \frac{21}{8}\right)$ & $363300 x^{6}-50652 x^{5}+128541 x^{4}+2266 x^{3}+19257 x^{2}+1008 x+896$ \\
$\left(\frac{38}{65},-\frac{13}{40}\right)$ & $-1106244 x^{6}+336780 x^{5}+23283 x^{4}+248770 x^{3}-101625 x^{2}$ \\
$\left(-\frac{8}{39},-\frac{1}{42}\right)$ & $-48600 x^{6}+483840 x^{5}-1386285 x^{4}-264482 x^{3}-282489 x^{2}$ \\
& \\
$\left(-\frac{70}{13},-\frac{21}{22}\right)$ & $599697 x^{6}-445662 x^{5}+824913 x^{4}-838612 x^{3}+2057823 x^{2}$ \\
$\left(-\frac{10}{7},-\frac{13}{36}\right)$ & $-1112220 x^{6}+2309556 x^{5}-397465 x^{4}-269262 x^{3}-847153 x^{2}$ \\
& $-265908 x+612$ \\
\hline
\end{tabular}

Table 26. Some rational points $(r, s)$ of small height on the surface of Theorem 41 and the corresponding genus- 2 curves. 
$s=-\frac{5}{6}$ gives a genus- 1 curve

$$
y^{2}=-\left(9 r^{2}+50 r+40\right)\left(243 r^{2}+670 r-40\right) .
$$

It has rational points, such as $(r, y)=(0,40)$. It is thus an elliptic curve; we find that it has conductor $2 \cdot 3 \cdot 5^{2} \cdot 29 \cdot 53$ and Mordell-Weil group $(\mathbb{Z} / 2 \mathbb{Z}) \oplus \mathbb{Z}^{2}$.

Pulling back sections of the elliptic fibration on the quotient surface gives us some more curves of genus 1 , each with a rational point and rank 1:

$$
\begin{array}{ccccc}
\multicolumn{2}{c}{\text { Equation }} & \text { point } & \text { conductor } & \text { group } \\
-\frac{4 t^{2}-5 t+2}{2 t(t-1)} & \frac{(2 t-1)\left(14 t^{3}-25 t^{2}+16 t-4\right)}{4 t^{2}(t-1)^{2}} & t=\frac{1}{2} & 53 & \mathbb{Z} \\
-\frac{12 t^{2}-5 t+2}{2 t(3 t-2)} & \frac{252 t^{4}-192 t^{3}+73 t^{2}-20 t+4}{4 t^{2}(3 t-2)^{2}} & t=0 & 2 \cdot 3 \cdot 4391 & \mathbb{Z} \\
-\frac{8 t^{2}-15 t+8}{2 t(t-1)} & \frac{92 t^{4}-320 t^{3}+433 t^{2}-268 t+64}{4 t^{2}(t-1)^{2}} & t=0 & 7 \cdot 977 & (\mathbb{Z} / 2 \mathbb{Z}) \oplus \mathbb{Z} \\
-\frac{3 t^{2}-3 t+2}{3 t(t-1)} & \frac{18 t^{4}-27 t^{3}+24 t^{2}-15 t+4}{9 t^{2}(t-1)^{2}} & t=0 & 2^{5} 3^{2} 7 & (\mathbb{Z} / 2 \mathbb{Z}) \oplus \mathbb{Z}
\end{array}
$$

\section{Discriminant 89}

32.1. Parametrization. We start with an elliptic K3 surface with fibers of type $A_{8} A_{7}$, and a section of height $\frac{89}{72}=4-\frac{1 \cdot 8}{9}-\frac{3 \cdot 5}{8}$.

The Weierstrass equation for this family is

$$
\begin{aligned}
& y^{2}=x^{3}+\left(a_{0}+a_{1} t+a_{2} t^{2}+a_{3} t^{3}+a_{4} t^{4}\right) x^{2} \\
&+ 2 \mu t^{2}\left(b_{0}+b_{1} t+b_{2} t^{2}+b_{3} t^{3}\right) x+\mu^{2} t^{4}\left(c_{0}+c_{1} t+c_{2} t^{2}\right),
\end{aligned}
$$

with

$$
\begin{aligned}
& a_{0}=(r s+1)^{2}, \quad b_{0}=-(r s+1)^{2}, \quad c_{0}=(r s+1)^{2}, \\
& \mu=4 r s(r+1)^{2}, \quad b_{3}=s\left(s^{2}-r s-2 s+1\right)^{2}, \quad a_{4}=s^{2}\left(s^{2}-r s-2 s+1\right)^{2}, \\
& c_{2}=\left(s^{2}-r s-2 s+1\right)^{2} \text {, } \\
& c_{1}=2 r s^{3}-2\left(r^{2}+4 r+1\right) s^{2}+4(r+1)^{2} s-2 \text {, } \\
& b_{2}=(2 r-1) s^{4}-\left(3 r^{2}+7 r-2\right) s^{3}+\left(r^{3}+6 r^{2}+7 r-1\right) s^{2}-2 r(r+1) s+r \text {, } \\
& a_{1}=-2\left(r^{2}-r\right) s^{3}+2\left(r^{3}-6 r-1\right) s^{2}+2\left(4 r^{2}+6 r+1\right) s+2 r \text {, } \\
& b_{1}=r(r-2) s^{3}-\left(r^{3}-r^{2}-10 r-2\right) s^{2}-\left(6 r^{2}+10 r+3\right) s-r+1 \text {, } \\
& a_{3}=2 s\left((r-1) s^{4}-\left(2 r^{2}+3 r-3\right) s^{3}+\left(r^{3}+4 r^{2}+3 r-3\right) s^{2}-\left(2 r^{2}+2 r-1\right) s+r\right) \text {, }
\end{aligned}
$$




$$
\begin{aligned}
a_{2}=\left(r^{2}-4 r+1\right) s^{4}-2\left(r^{3}-2\right. & \left.r^{2}-9 r\right) s^{3} \\
& +\left(r^{4}-16 r^{2}-22 r-3\right) s^{2}-2\left(r^{3}-r-1\right) s+r^{2} .
\end{aligned}
$$

To obtain an $E_{8} E_{7}$ elliptic fibration on these $\mathrm{K} 3$ surfaces, we first move by a 2-neighbor step to one with $E_{7}$ and $A_{8}$ fibers.

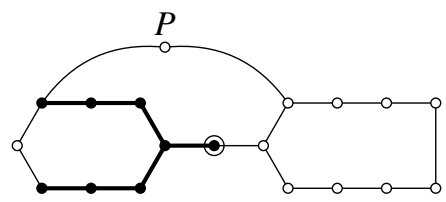

The elliptic fibration defined by this new fiber $F^{\prime}$ has a section, since $P \cdot F^{\prime}=1$. Also, the new elliptic fibration must have a section $P^{\prime}$ of height $\frac{89}{18}=4+2 \cdot 2-\frac{3}{2}-\frac{2 \cdot 7}{9}$.

Finally, we go to $E_{8} E_{7}$ by a 3-neighbor step.

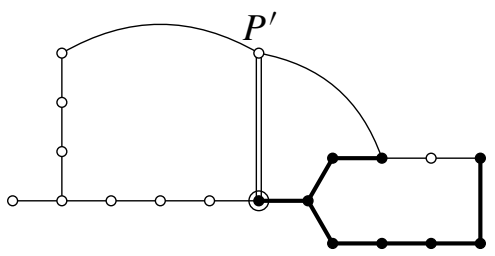

The new fiber $F^{\prime \prime}$ satisfies $P^{\prime} \cdot F^{\prime \prime}=2+2 \cdot 3=8$, and the identity component of the $E_{7}$ fiber intersects $F^{\prime}$ in 3 . Since these have greatest common divisor 1, the genus-1 fibration defined by $F^{\prime}$ has a section.

Theorem 42. A birational model over $\mathbb{Q}$ for the Hilbert modular surface $Y_{-}(89)$ as a double cover of $\mathbb{P}_{r, s}^{2}$ is given by the equation

$$
\begin{aligned}
z^{2}=s^{4} & r^{6}-2 s^{3}\left(2 s^{2}+3 s+2\right) r^{5}+s^{2}\left(6 s^{4}+16 s^{3}-49 s^{2}-26 s+6\right) r^{4} \\
& -2 s\left(2 s^{6}+6 s^{5}-50 s^{4}+26 s^{3}+73 s^{2}-35 s+2\right) r^{3} \\
& +\left(s^{8}-36 s^{6}+26 s^{5}+273 s^{4}-514 s^{3}+271 s^{2}-38 s+1\right) r^{2} \\
& +2(s-1)^{2} s\left(s^{5}-4 s^{4}+25 s^{3}-107 s^{2}+147 s-44\right) r+(s-4)^{3}(s-1)^{4} s .
\end{aligned}
$$

It is a surface of general type.

32.2. Analysis. The branch locus has genus 1; one can give an explicit isomorphism (see the online supplement) to the elliptic curve of conductor 89 given by the Weierstrass equation

$$
y^{2}+x y+y=x^{3}+x^{2}-x .
$$

It is isomorphic to $X_{0}(89) /\langle w\rangle$, where $w$ is the Atkin-Lehner involution.

The Hilbert modular surface $Y_{-}(89)$ is a surface of general type. Note that the change of coordinates $r=s+g$ simplifies the equation a bit further, making the 


\begin{tabular}{|c|c|}
\hline$(r, s)$ & Sextic polynomial $f_{6}(x)$ defining the genus- 2 curve \\
\hline$\left(-\frac{31}{3},-\frac{1}{6}\right)$ & $-334084 x^{6}+65892 x^{5}+847841 x^{4}-156012 x^{3}-1036555 x^{2}-453867 x-525$ \\
\hline$\left(-\frac{49}{9},-\frac{2}{3}\right)$ & $632 x^{6}-480 x^{5}+43475 x^{4}-97578 x^{3}-1030393 x^{2}+855708 x-1045044$ \\
\hline$(-19,-1)$ & $\begin{aligned} & 126905 x^{6}+2388081 x^{5}-2600778 x^{4}-3075787 x^{3}-5448045 x^{2} \\
&-3683352 x-709200\end{aligned}$ \\
\hline$\left(-\frac{31}{10}, \frac{5}{2}\right)$ & $\begin{array}{r}-83300 x^{6}+168420 x^{5}+5079215 x^{4}-6586832 x^{3}+584735 x^{2} \\
+70020 x-8100\end{array}$ \\
\hline$\left(-\frac{5}{6}, \frac{11}{6}\right)$ & $\begin{aligned} 2185004 x^{6}-12346980 x^{5}+10798163 x^{4}+732660 x^{3} & +47975267 x^{2} \\
& +21406020 x+27911916\end{aligned}$ \\
\hline$\left(\frac{13}{9},-\frac{5}{9}\right)$ & $\begin{array}{r}-14966100 x^{6}-43598124 x^{5}+25890735 x^{4}+105396908 x^{3}-44422995 x^{2} \\
-65750574 x+34674550\end{array}$ \\
\hline$\left(-\frac{40}{7},-\frac{1}{2}\right)$ & $\begin{array}{r}2754000 x^{6}+86434200 x^{5}+150411025 x^{4}-14830346 x^{3}-49970411 x^{2} \\
+242599308 x+131021492\end{array}$ \\
\hline$\left(\frac{16}{33}, \frac{11}{3}\right)$ & $\begin{array}{r}25329267 x^{6}-96789717 x^{5}+223774305 x^{4}-449560367 x^{3}-46904988 x^{2} \\
-772810308 x+413626230\end{array}$ \\
\hline
\end{tabular}

Table 27. Some rational points $(r, s)$ of small height on the surface of Theorem 42 and the corresponding genus- 2 curves.

degree of the right-hand side equal to 6 in each variable. However, it complicates the original defining Weierstrass equation of the family of K3 surfaces, so we have chosen the $(r, s)$ coordinate system.

32.3. Examples. Table 27 lists some points of small height and their genus-2 curves.

We find two elliptic curves of positive rank on the surface. The specialization $s=\frac{25}{22}$ gives a curve of genus 1

$$
y^{2}=1210000 r^{4}-19157600 r^{3}-17065736 r^{2}+678600 r-8575
$$

with rational points (as at infinity), conductor $3 \cdot 5 \cdot 11 \cdot 163 \cdot 191 \cdot 881$, and rank at least 2. The locus $s=r+\frac{101}{50}$ gives another curve of genus 1 ,

$$
y^{2}=-3739190000 r^{4}-21451957600 r^{3}-43018833576 r^{2}
$$

$$
-36551728152 r-11227811551 \text {, }
$$

with rational points (as at $(r, y)=\left(-1, \pm 65^{2}\right)$ ), conductor $2^{4} \cdot 5 \cdot 17 \cdot 19 \cdot 463 \cdot 58787$, and rank at least 3 . We were not able to determine the exact rank of either curve, but the global root numbers indicate that the rank should be even for the former curve and odd for the latter, so one might guess that the lower bounds 2 and 3 on their ranks are sharp. 


\section{Discriminant 92}

33.1. Parametrization. We start with an elliptic K3 surface with fibers of type $A_{8}, A_{1}$ and $D_{6}$, and a section of height $\frac{92}{72}=\frac{23}{18}=4-\frac{1 \cdot 1}{2}-\frac{4 \cdot 5}{9}$.

The Weierstrass equation may be written as

$$
\begin{aligned}
& y^{2}=x^{3}+\left(a_{0}+a_{1} t+a_{2} t^{2}+a_{3} t^{3}\right) x^{2} \\
&+ 2 t^{2}(\lambda t-\mu)\left(b_{0}+b_{1} t+b_{2} t^{2}\right) x+t^{4}(\lambda t-\mu)^{2}\left(c_{0}+c_{1} t\right),
\end{aligned}
$$

with

$$
\begin{array}{rlrl}
\lambda & =(r+s)^{2}, & \mu & =r+2 s, \\
a_{3} & =-4 r s\left(s+r^{2}+r\right)\left(s^{2}+r s-r\right), & a_{0} & =(r s-r-1)^{2}, \\
b_{0} & =-4 r s^{2}(r s-r-1)^{2}, & c_{0} & =16 r^{2} s^{4}(r s-r-1)^{2}, \\
a_{1} & =-2 r\left((r+1)^{2}(s-1)^{2}+s^{2}\right), & b_{1} & =4 r^{2} s^{2}(r s+2 s-r-1)^{2}, \\
c_{1} & =-64 r^{3}(r+1)(s-1) s^{5}, & \\
b_{2} & =-8 r^{2} s^{3}\left((r+2) s^{2}+\left(2 r^{2}+2 r-1\right) s-2 r(r+1)\right), \\
a_{2} & =r(r s+4 s-r-1)\left(4 s^{2}+r^{2} s+4 r s-r^{2}-r\right) .
\end{array}
$$

As in the case of discriminant 56, we first go to an $E_{7} A_{8}$ fibration using the $E_{7}$ fiber $F^{\prime}$ identified below. Note that $F^{\prime} \cdot P=3$, while the component of the $D_{6}$ fiber which is not included in $F^{\prime}$ intersects $F^{\prime}$ with multiplicity 2 . Since $\operatorname{gcd}(2,3)=1$, the fibration defined by $F^{\prime}$ has a section.

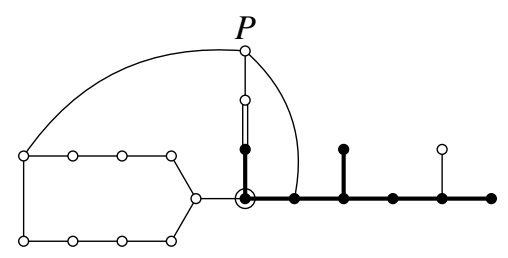

The new elliptic fibration has a section $P^{\prime}$ of height $\frac{92}{2 \cdot 9}=\frac{46}{9}=4+2 \cdot 1-\frac{8}{9}$, which must therefore intersect the zero section, the identity component of the $E_{7}$ fiber and component 1 of the $A_{8}$ fiber.

We identify an $E_{8}$ fiber and compute its Weierstrass equation by a 3-neighbor move. Note that it intersects $P^{\prime}$ in 7 and the excluded component of the $A_{8}$ fiber in 3. Therefore the fibration it defines has a section, and we may convert to the Jacobian.

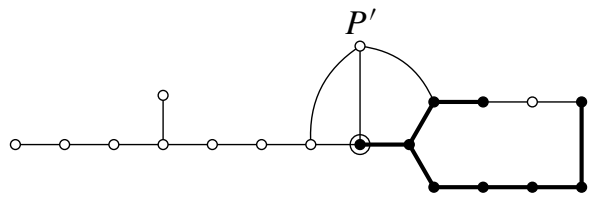


Now we read out the Igusa-Clebsch invariants and compute the branch locus.

Theorem 43. A birational model over $\mathbb{Q}$ for the Hilbert modular surface $Y_{-}(92)$ as a double cover of $\mathbb{P}_{r, s}^{2}$ is given by the equation

$$
\begin{aligned}
z^{2}= & \left(s+r^{2}+r\right)\left(s^{2}+r s-r\right) \\
& \times\left((s-1)^{3} r^{5}+(s-1)^{2}\left(s^{2}-15 s-3\right) r^{4}-(s-1)\left(42 s^{3}-27 s^{2}-31 s-3\right) r^{3}\right. \\
& \left.\quad-\left(27 s^{5}-30 s^{4}-77 s^{3}+69 s^{2}+17 s+1\right) r^{2}+s\left(46 s^{3}-30 s^{2}-42 s-1\right) r-27 s^{3}\right) .
\end{aligned}
$$

It is a surface of general type.

33.2. Analysis. The branch locus has three components. Points of the two simpler components correspond to elliptic K3 surfaces where the $D_{6}$ fiber is promoted to an $E_{7}$ fiber, while the more complicated component corresponds to an extra $\mathrm{I}_{2}$ fiber. All the three components are rational (genus 0 ). This is obvious from inspection for the simpler components, and for the last we have the parametrization

$$
(r, s)=\left(\frac{t\left(t^{2}+2\right)(t+1)^{2}}{(t+2)\left(t^{3}+2 t^{2}+2 t+2\right)}, \frac{-t(t+1)\left(t^{3}+2 t^{2}+4 t+4\right)}{(t+2)^{2}\left(t^{2}+2\right)}\right) .
$$

The surface $Y_{-}(92)$ is a surface of general type. The extra involution is $(r, s, z) \mapsto$ $\left(-1 / s,-1 / r, z /(r s)^{4}\right)$. We now analyze the quotient of the Hilbert modular surface by this involution. Because the involution fixes $r / s$, we obtain the quotient by setting $r=s t$ and writing everything in terms of $m=s-1 /(t s)$. We find the equation

$$
\begin{array}{r}
y^{2}=\left(t^{2}(t+1) m-t^{3}+t^{2}+2 t+1\right)\left(t^{3}(t+1) m^{3}-t\left(3 t^{3}+17 t^{2}+42 t+27\right) m^{2}\right. \\
\left.+t\left(3 t^{3}+31 t^{2}+72 t+30\right) m-\left(t^{4}+15 t^{3}+30 t^{2}+7 t+8\right)\right),
\end{array}
$$

which expresses the quotient as a genus- 1 curve over $\mathbb{Q}(t)$. Since there is an obvious section (where the first factor vanishes), we may convert to the Jacobian, which has the Weierstrass equation (after shifting $t$ by 1 and performing some Weierstrass transformations)

$$
\begin{aligned}
y^{2}=x^{3}-(2 t+1)\left(8 t^{3}+8 t^{2}-6 t-1\right) x^{2} & \\
& -8(t-1) t^{4}(t+1)\left(10 t^{2}-32 t-5\right) x-16(t-1)^{2} t^{8}(t+1)(47 t+7) .
\end{aligned}
$$

This is an elliptic K3 surface. It has reducible fibers of type $\mathrm{I}_{8}$ at $t=0, \mathrm{I}_{3}$ at 1 and $(-7 \pm 3 \sqrt{3}) / 11$, and $\mathrm{I}_{2}$ at the roots of $11 t^{3}-10 t^{2}+5 t+1$ (which generates the cubic field of discriminant -23 ). Therefore the trivial lattice has rank 18 . We easily identify a non-torsion section $P$ of height $\frac{5}{8}$ with $x$-coordinate $4 t^{3}(6 t+1)$. On the other hand, counting points modulo 13 and 17 shows that the Picard number cannot be 20. Therefore the Picard number of this quotient surface is 19. The sublattice of the Néron-Severi group spanned by $P$ and the trivial lattice has discriminant $360=2^{3} \cdot 3^{2} \cdot 5$. It is easy to see from height calculations that there cannot be any 2 - 


\begin{tabular}{c|c}
$(r, s)$ & Sextic polynomial $f_{6}(x)$ defining the genus-2 curve $y^{2}=f_{6}(x)$ \\
\hline$\left(\frac{3}{10},-\frac{39}{70}\right)$ & $5981584 x^{6}-4016376 x^{5}+1699985 x^{4}+\begin{array}{r}313485 x^{3}-168322 x^{2} \\
+49665 x+21175\end{array}$ \\
$\left(\frac{70}{39},-\frac{10}{3}\right)$ & $\begin{array}{r}2916 x^{6}+591516 x^{5}+6670933 x^{4}+12740602 x^{3}-44084051 x^{2} \\
+8704740 x+16105100\end{array}$ \\
\hline
\end{tabular}

Table 28. Some rational points $(r, s)$ of small height on the surface of Theorem 43 and the corresponding genus-2 curves.

or 3-torsion sections, and that $P$ cannot be divisible by 2 or 3 in the Mordell-Weil group. Hence, this sublattice is the entire Néron-Severi lattice.

33.3. Examples. Table 28 lists some points of small height and their genus- 2 curves.

Specializations of $r$ or $s$, and pullbacks of sections of the quotient, do not seem to yield any genus 0 or 1 curves on the surface (at any rate, none corresponding to abelian surfaces with "honest" real multiplication by $\mathcal{O}_{92}$ and not a larger endomorphism ring).

\section{Discriminant 93}

34.1. Parametrization. Start with an elliptic K3 surface with fibers of type $A_{10}$, $A_{3}$ and $A_{2}$, with a section of height $\frac{31}{44}=4-\frac{3}{4}-\frac{28}{11}$. The extra involution comes from flipping the $A_{2}$ fiber.

This family has the Weierstrass equation

$$
\begin{aligned}
y^{2}=x^{3}+ & \left(a_{0}+a_{1} t+a_{2} t^{2}+a_{3} t^{3}+a_{4} t^{4}\right) x^{2} \\
& +2 t(\lambda t-\mu)\left(b_{0}+b_{1} t+b_{2} t^{2}+b_{3} t^{3}\right) x+t^{2}(\lambda t-\mu)^{2}\left(c_{0}+c_{1} t+c_{2} t^{2}\right),
\end{aligned}
$$

with

$$
\begin{array}{rlrl}
\lambda & =-\left(n^{2}-1\right), & \mu & =\left(n^{2}-m n-n-m\right)\left(n^{2}+m n+n-m\right), \\
a_{0} & =(m+1)^{2} n^{8}, & b_{0} & =m^{4}(m+1)^{2} n^{8}, \\
c_{0} & =m^{8}(m+1)^{2} n^{8}, & a_{4} & =1, \\
c_{2} & =m^{8} n^{4}, & b_{3} & =m^{4} n^{2}, \\
a_{3} & =\left(m^{2}+2 m+4\right) n^{2}-3 m^{2}, & c_{1}=m^{8} n^{4}\left(\left(m^{2}+2 m+2\right) n^{2}-m^{2}\right), \\
b_{2} & =m^{4} n^{2}\left(\left(m^{2}+2 m+3\right) n^{2}-2 m^{2}\right), \\
a_{2} & =3\left(m^{2}+2 m+2\right) n^{4}-2 m^{2}\left(m^{2}+3 m+3\right) n^{2}+3 m^{4}, \\
b_{1} & =m^{4} n^{2}\left(\left(2 m^{2}+4 m+3\right) n^{4}-m^{2}(m+1)(m+2) n^{2}+m^{4}\right), \\
a_{1} & =\left(3 m^{2}+6 m+4\right) n^{6}-3 m^{2}(m+1)^{2} n^{4}+m^{4}(m+1)(m+3) n^{2}-m^{6} .
\end{array}
$$


We first identify the class of an $E_{8}$ fiber below, and move to it by a 3-neighbor step.

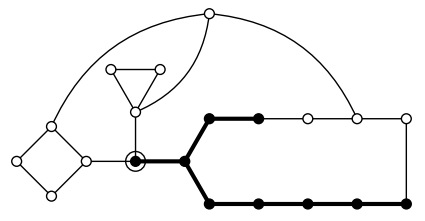

This gives us an elliptic fibration with $E_{8}, A_{5}$ and $A_{2}$ fibers, and a section $P$ of height $\frac{93}{18}=\frac{31}{6}=4+2 \cdot 1-\frac{5}{6}$. We then identify an $E_{7}$ fiber and move to it by a 2-neighbor step. Since the new fiber intersects the section $P$ in 7 and the excluded component of the $A_{5}$ fiber in 3 , we see that the new fibration has a section.

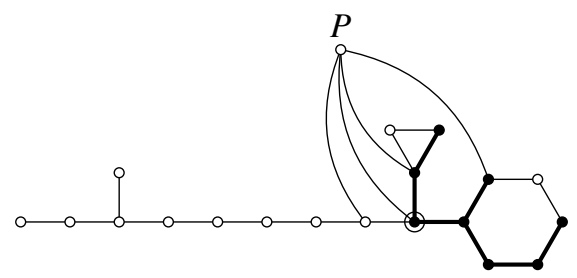

Theorem 44. A birational model over $\mathbb{Q}$ for the Hilbert modular surface $Y_{-}(93)$ as a double cover of $\mathbb{P}_{m, n}^{2}$ is given by the equation

$$
\begin{aligned}
z^{2}=16 & \left(n^{2}-1\right)^{2} n^{2} m^{6}+8\left(n^{2}-1\right)\left(21 n^{4}+22 n^{2}-27\right) m^{5} \\
& -\left(27 n^{8}-684 n^{6}-1246 n^{4}+1620 n^{2}+27\right) m^{4} \\
& -8 n^{2}\left(27 n^{6}-109 n^{4}-471 n^{2}+41\right) m^{3}-8 n^{2}\left(81 n^{6}+135 n^{4}-273 n^{2}-7\right) m^{2} \\
& -96 n^{4}\left(9 n^{2}-1\right)\left(n^{2}+3\right) m-16 n^{4}\left(n^{2}+3\right)\left(27 n^{2}+1\right) .
\end{aligned}
$$

It is a surface of general type.

34.2. Analysis. The extra involution is $\iota:(m, n) \mapsto(m,-n)$.

The branch locus is a curve of genus 4 , isomorphic to $X_{0}(93) /\left\langle w_{93}\right\rangle$, where $w_{93}$ is the Atkin-Lehner involution. We do not give the explicit isomorphism here, but the formulas are available in the online supplement. Setting $k=n^{2}$, we can write it as a double cover of a genus- 2 curve, which can be transformed to the Weierstrass form

$$
y^{2}-\left(9 x^{3}+11 x-3\right) y+x^{2}\left(69 x^{3}-56 x^{2}+81 x-22\right)=0 .
$$

This Hilbert modular surface is a surface of general type. The quotient by this involution $\iota$ has the equation (with $k=n^{2}$ )

$$
\begin{aligned}
z^{2}=-27(m+2)^{4} & k^{4}+4\left(4 m^{6}+42 m^{5}+171 m^{4}+218 m^{3}-270 m^{2}-624 m-328\right) k^{3} \\
& -2\left(16 m^{6}-4 m^{5}-623 m^{4}-1884 m^{3}-1092 m^{2}-144 m+24\right) k^{2} \\
+ & 4 m^{2}\left(4 m^{4}-98 m^{3}-405 m^{2}-82 m+14\right) k+27 m^{4}(8 m-1) .
\end{aligned}
$$


This has a genus-1 fibration over $\mathbb{Q}(m)$. The fibration has a section at infinity defined over $\mathbb{Q}(\sqrt{-3})$; we do not know whether there is a section defined over $\mathbb{Q}$. The Jacobian has the Weierstrass equation

$$
\begin{aligned}
y^{2}=x^{3}+\left(m^{6}+20 m^{5}+118 m^{4}+\right. & \left.186 m^{3}+33 m^{2}+18 m-3\right) x^{2} \\
+ & 8 m^{2}(m+10)\left(9 m^{4}+39 m^{3}+57 m^{2}-1\right) x \\
+ & 16 m^{4}(m+10)^{2}\left(4 m^{3}+13 m^{2}+18 m-3\right) .
\end{aligned}
$$

This is an honestly elliptic surface with $\chi=3$. It has reducible fibers of type $\mathrm{I}_{9}$ at $m=\infty, \mathrm{I}_{4}$ at $m=0, \mathrm{I}_{2}$ at $m=-10$, and $\mathrm{I}_{3}$ at the roots of

$$
m^{6}+11 m^{5}+16 m^{4}+32 m^{3}+17 m^{2}-9 m+1
$$

(whose splitting field is a dihedral extension of degree 12 containing $\sqrt{93}$ ). The trivial lattice has rank 26, leaving room for Mordell-Weil rank up to 4. Counting points modulo 13 and 17 shows that the Picard number is at most 29. On the other hand, we are (so far) able to produce the sections

$$
\begin{aligned}
& P_{0}=\left(-16\left(m^{3}+m^{2}+6 m-1\right), 32\left(m^{6}+11 m^{5}+16 m^{4}+32 m^{3}+17 m^{2}-9 m+1\right)\right), \\
& P_{1}=\left(-16(m+10)\left(m^{5}+m^{4}+6 m^{3}+3 m^{2}+18 m-3\right) / 31,\right. \\
& \left.288(m+10)\left(3 m^{2}+2 m+9\right)\left(m^{6}+11 m^{5}+16 m^{4}+32 m^{3}+17 m^{2}-9 m+1\right) / 93^{3 / 2}\right),
\end{aligned}
$$

of which $P_{0}$ is 3-torsion, while $P_{1}$ has height $\frac{3}{2}$. Therefore, the Mordell-Weil rank is between 1 and 3 .

34.3. Examples. Table 29 lists some points of small height and their genus-2 curves.

\begin{tabular}{l|c}
$(m, n)$ & Sextic polynomial $f_{6}(x)$ defining the genus-2 curve $y^{2}=f_{6}(x)$ \\
\hline$\left(2, \frac{1}{3}\right)$ & $-2112 x^{6}-5184 x^{5}+5451 x^{4}+2593 x^{3}-4596 x^{2}-2223 x-101$ \\
$\left(-10, \frac{5}{3}\right)$ & $-7452 x^{6}-4860 x^{5}-24039 x^{4}-4540 x^{3}-17205 x^{2}+4686 x-302$ \\
$\left(\frac{1}{5}, \frac{1}{7}\right)$ & $-24786 x^{6}+25272 x^{5}+90900 x^{4}-73885 x^{3}-107482 x^{2}+54020 x+40286$ \\
$(-10,5)$ & $-31752 x^{5}-48825 x^{4}+52868 x^{3}-175537 x^{2}+91124 x-80644$ \\
$\left(2,-\frac{1}{3}\right)$ & $-594 x^{6}+10962 x^{5}-154233 x^{4}+391936 x^{3}+265521 x^{2}+330228 x-71068$ \\
$(-10,-5)$ & $43756 x^{6}+110088 x^{5}+463887 x^{4}-609201 x^{3}+208770 x^{2}-6300 x-211000$ \\
$\left(-10,-\frac{5}{3}\right)$ & $-3008 x^{6}+270048 x^{5}-773739 x^{4}-611989 x^{3}-2150523 x^{2}$ \\
& $+631152 x-342144$ \\
$\left(\frac{1}{5},-\frac{1}{7}\right)$ & $-253800 x^{6}-1186380 x^{5}-1627302 x^{4}+4611739 x^{3}+1795017 x^{2}$
\end{tabular}

Table 29. Some rational points $(m, n)$ of small height on the surface of Theorem 44 and the corresponding genus- 2 curves. 


\section{Discriminant 97}

35.1. Parametrization. Start with an elliptic K 3 surface with fibers of type $D_{5}, A_{4}$ and $A_{6}$, with a section of height $\frac{97}{140}=4-\left(1+\frac{1}{4}\right)-\frac{6}{5}-\frac{6}{7}$.

We can write the Weierstrass equation as

$$
\begin{aligned}
y^{2}=x^{3}+\left(a_{0}+a_{1} t+a_{2} t^{2}+a_{3} t^{3}\right) x^{2}+2 t^{2}(t-1)^{2}\left(b_{0}+b_{1} t\right. & \left.+b_{2} t^{2}\right) x \\
& +t^{4}(t-1)^{4}\left(c_{0}+c_{1} t\right),
\end{aligned}
$$

with

$$
\begin{aligned}
& a_{0}=(r+1)^{2}\left(r s^{2}+s^{2}+r^{2} s+r\right)^{2}, \\
& a_{1}=2(r+1)\left((r+1)^{2} s^{5}+2(r+1)\left(r^{2}-r-1\right) s^{4}+r\left(r^{3}-4 r^{2}-4 r+2\right) s^{3}\right. \\
& \left.-r\left(2 r^{3}+r^{2}+2 r+5\right) s^{2}-r^{2}\left(r^{2}+3 r+3\right) s-r^{2}(r+1)\right), \\
& a_{2}=(r+1)^{2} s^{6}+2(r+1)\left(r^{2}-4 r-2\right) s^{5}+\left(r^{4}-16 r^{3}-6 r^{2}+18 r+4\right) s^{4} \\
& -2 r\left(4 r^{3}-6 r^{2}-4 r+11\right) s^{3}+r\left(6 r^{3}-6 r^{2}-3 r+20\right) s^{2} \\
& +2 r^{2}(r+3)(2 r+3) s+r^{2}(r+1)^{2}, \\
& a_{3}=-4 r(s-1) s(s+r)\left(s^{3}+(r-2) s^{2}-(r-2) s-2 r-3\right) \text {, } \\
& b_{0}=4 r(r+1)^{2}(s-1)^{2} s(s+r)\left(r s^{2}+s^{2}+r^{2} s+r\right)^{2} \text {, } \\
& b_{1}=4 r(r+1)(s-1)^{2} s(s+r) \\
& \times\left((r+1)^{2} s^{5}+2(r+1)\left(r^{2}-2 r-1\right) s^{4}+r\left(r^{3}-8 r^{2}-6 r+4\right) s^{3}\right. \\
& \left.-r\left(4 r^{3}+r^{2}+2 r+7\right) s^{2}-r^{2}\left(r^{2}+5 r+5\right) s-r^{2}(r+1)\right), \\
& b_{2}=-8 r^{2}(r+1)(s-1)^{2} s^{2}(s+r)^{2}\left(2 s^{3}+2(r-2) s^{2}-2(r-2) s-r-3\right) \text {, } \\
& c_{0}=16 r^{2}(r+1)^{2}(s-1)^{4} s^{2}(s+r)^{2}\left(r s^{2}+s^{2}+r^{2} s+r\right)^{2} \text {, } \\
& c_{1}=-64 r^{3}(r+1)^{2}(s-1)^{4} s^{3}(s+r)^{3}\left(s^{2}+r s-s+1\right) \text {. }
\end{aligned}
$$

First we identify a $D_{8}$, and move to the associated elliptic fibration (which also has an $A_{6}$ fiber) by a 2-neighbor step.

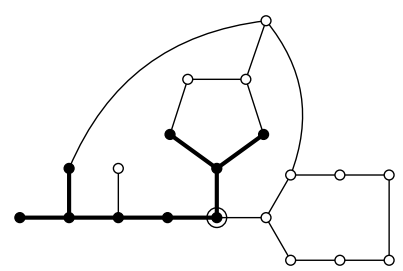

This elliptic fibration has $D_{8}$ and $A_{6}$ fibers, and two independent sections $P, Q$ with height matrix

$$
\left(\begin{array}{rr}
\frac{8}{7} & -\frac{5}{14} \\
-\frac{5}{14} & \frac{22}{7}
\end{array}\right) \text {. }
$$


We identify a fiber $F^{\prime}$ of type $E_{8}$ and move to the associated fibration by a 2-neighbor step. Note that $Q \cdot F^{\prime}=3$, while the remaining component of the $D_{8}$ fiber has intersection 2 with $F^{\prime}$. Therefore the new genus-1 fibration has a section.

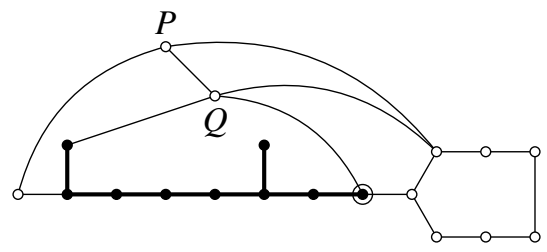

The new elliptic fibration has bad fibers of types $E_{8}$ and $A_{7}$, and a section $P^{\prime}$ of height $\frac{97}{8}=2+2 \cdot 6-3 \cdot \frac{5}{8}$. We identify a fiber $F^{\prime \prime}$ of type $E_{7}$, and move to the associated elliptic fibration by a 2-neighbor step. Note that $P^{\prime} \cdot F^{\prime \prime}=13$, while the remaining component of the $A_{7}$ fiber has intersection 2 with $F^{\prime \prime}$. Therefore the elliptic fibration associated to $F^{\prime \prime}$ has a section, and is the of the desired type $E_{8} E_{7}$.

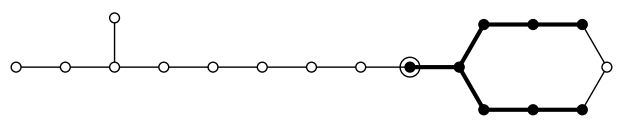

We now read out the Igusa-Clebsch invariants and work out the equation of the branch locus of $Y_{-}(97)$ as a double cover of $\mathcal{H}_{97}$.

Theorem 45. A birational model over $\mathbb{Q}$ for the Hilbert modular surface $Y_{-}(97)$ as a double cover of $\mathbb{P}_{r, s}^{2}$ is given by the equation

$$
\begin{aligned}
z^{2}=s^{2}\left(s^{2}+14\right. & +1) r^{6}+2 s\left(2 s^{4}+27 s^{3}-13 s^{2}+15 s+1\right) r^{5} \\
& +\left(6 s^{6}+80 s^{5}-75 s^{4}+128 s^{3}-54 s^{2}+18 s+1\right) r^{4} \\
& +2\left(2 s^{7}+28 s^{6}-32 s^{5}+84 s^{4}-74 s^{3}+48 s^{2}-13 s+1\right) r^{3} \\
& +\left(s^{8}+18 s^{7}-11 s^{6}+68 s^{5}-101 s^{4}+112 s^{3}-69 s^{2}+22 s+1\right) r^{2} \\
& +2 s^{2}\left(s^{6}+3 s^{5}-5 s^{4}+7 s^{3}+3 s^{2}-16 s+12\right) r+(s-2)^{4} s^{4} .
\end{aligned}
$$

It is a surface of general type.

35.2. Analysis. The branch locus is a genus- 3 curve, isomorphic to the quotient of $X_{0}(97)$ by the Atkin-Lehner involution. We omit the formulas for the isomorphism, but they are available in the online supplement.

The Hilbert modular surface itself is of general type. The substitution $r=u-s$ simplifies the equation of the double cover somewhat, making it degree- 6 in each variable. However, it complicates the original Weierstrass equation of the K3 family, so we have chosen the $(r, s)$-coordinates on the moduli space. 


\begin{tabular}{l|c}
$(r, s)$ & Sextic polynomial $f_{6}(x)$ defining the genus-2 curve $y^{2}=f_{6}(x)$ \\
\hline$\left(\frac{5}{2},-2\right)$ & $-100 x^{6}+180 x^{5}+3 x^{4}+12 x^{3}-207 x^{2}+54 x+54$ \\
$\left(-\frac{5}{3}, \frac{2}{3}\right)$ & $115 x^{6}-120 x^{5}-692 x^{4}-42 x^{3}+643 x^{2}+36 x-156$ \\
$\left(\frac{8}{15},-\frac{6}{5}\right)$ & $-418 x^{6}-99 x^{5}+700 x^{4}+130 x^{3}-401 x^{2}-45 x+81$ \\
$\left(-\frac{14}{3}, 6\right)$ & $528 x^{6}-792 x^{5}+311 x^{4}-26 x^{3}-205 x^{2}+60 x-20$ \\
$\left(-\frac{20}{7}, \frac{5}{14}\right)$ & $-72 x^{6}-72 x^{5}+669 x^{4}+706 x^{3}-1623 x^{2}-60 x+500$ \\
$\left(\frac{13}{6},-\frac{3}{2}\right)$ & $1236 x^{6}-852 x^{5}-1919 x^{4}+1702 x^{3}+1473 x^{2}-940 x-700$ \\
$\left(\frac{17}{6},-\frac{1}{3}\right)$ & $200 x^{6}-420 x^{5}+1918 x^{4}-1455 x^{3}+2968 x^{2}+1740 x-1175$ \\
$\left(-\frac{13}{7}, \frac{11}{21}\right)$ & $-1872 x^{5}-3540 x^{4}+1021 x^{3}+2331 x^{2}-1185 x+145$ \\
$\left(\frac{23}{35},-\frac{5}{14}\right)$ & $370 x^{6}+1084 x^{5}-2510 x^{4}-683 x^{3}-32 x^{2}-752 x-3822$ \\
$\left(-\frac{11}{30}, \frac{5}{6}\right)$ & $-1225 x^{6}+3570 x^{5}-3266 x^{4}-176 x^{3}+3463 x^{2}+1446 x+5868$ \\
$\left(\frac{1}{2}, \frac{5}{2}\right)$ & $-1938 x^{6}+3132 x^{5}+1730 x^{4}-855 x^{3}+609 x^{2}-9065 x+5145$ \\
$\left(-\frac{19}{30}, \frac{3}{10}\right)$ & $4900 x^{6}+5320 x^{5}-11751 x^{4}-4255 x^{3}+2867 x^{2}+4515 x-1596$ \\
$\left(-\frac{29}{18}, \frac{10}{9}\right)$ & $16048 x^{6}-7524 x^{5}-11096 x^{4}+16107 x^{3}-4244 x^{2}-5652 x-864$ \\
$\left(\frac{13}{4},-\frac{1}{4}\right)$ & $-1140 x^{6}+4820 x^{5}-3105 x^{4}+3366 x^{3}-16681 x^{2}-6468 x-12348$ \\
$\left(-\frac{7}{2}, 6\right)$ & $14076 x^{6}-20748 x^{5}+11899 x^{4}+1252 x^{3}-125 x^{2}+2676 x+380$ \\
$\left(\frac{9}{10},-\frac{2}{5}\right)$ & $-6688 x^{6}+9840 x^{5}-8271 x^{4}+24640 x^{3}-5373 x^{2}+12150 x-7290$ \\
\hline
\end{tabular}

Table 30. Some rational points $(r, s)$ of small height on the surface of Theorem 45 and the corresponding genus- 2 curves.

35.3. Examples. Table 30 lists some points of small height and their genus- 2 curves.

We find a few curves with infinitely many rational points. For instance, $r=1-s$ gives a rational curve, with parametrization

$$
(r, s)=\left(\frac{(m+1)(m+3)}{m^{2}+7}, \frac{-4(m-1)}{m^{2}+7}\right) .
$$

The Brauer obstruction vanishes identically along this curve. However, it turns out to be a modular curve: the corresponding abelian surfaces have endomorphism ring a (split) quaternion algebra.

Another curve of genus 0 is given by $r=-\left(3 s^{2}+8 s+4\right) /(3 s)$. Again, the Brauer obstruction vanishes, and this time we get a family of abelian surfaces with "honest" real multiplication.

The locus $r=\frac{1}{2}-s$ gives a genus- 1 curve

$$
y^{2}=(2 s+1)\left(2 s^{3}-39 s^{2}+28 s+36\right)
$$

with conductor $5862=2 \cdot 3 \cdot 977$ and Mordell-Weil group $\mathbb{Z}^{2}$. 


\section{Acknowledgements}

We thank Jennifer Balakrishnan, Henri Darmon, Lassina Dembélé, Eyal Goren, Kiran Kedlaya, Ronen Mukamel, George Pappas, Bjorn Poonen, Frithjof Schulze, Matthias Schütt and Andrew Sutherland for helpful comments. We also thank the anonymous referee for a careful reading of the paper and several useful remarks. The computer algebra systems PARI/gp, Maple, Maxima, and Magma were used in the calculations for this paper. We also made heavy use of the programs mwrank, ratpoints, the Maple package algcurves, and the Magma program ConicsFF.m for finding points on conics over function fields. We thank the authors of these programs as well.

\section{References}

[An et al. 2001] S. Y. An, S. Y. Kim, D. C. Marshall, S. H. Marshall, W. G. McCallum, and A. R. Perlis, "Jacobians of genus one curves", J. Number Theory 90:2 (2001), 304-315. MR 2002g:14040 Zbl 1066.14035

[Birkenhake and Lange 2004] C. Birkenhake and H. Lange, Complex abelian varieties, 2nd ed., Grundlehren der Mathematischen Wissenschaften 302, Springer, Berlin, 2004. MR 2005c:14001 Zbl 1056.14063

[Brumer 1995] A. Brumer, "The rank of $J_{0}(N)$ ", pp. 3, 41-68 in Columbia University Number Theory Seminar (New York, 1992), Astérisque 228, Soc. Math. France, Paris, 1995. MR 96f:11083 Zbl 0851.11035

[Clingher and Doran 2007] A. Clingher and C. F. Doran, "Modular invariants for lattice polarized K3 surfaces”, Michigan Math. J. 55:2 (2007), 355-393. MR 2009a:14049 Zbl 1132.14035

[Deligne and Pappas 1994] P. Deligne and G. Pappas, "Singularités des espaces de modules de Hilbert, en les caractéristiques divisant le discriminant", Compositio Math. 90:1 (1994), 59-79. MR 95a:11041 Zbl 0826.14027

[Dembélé and Kumar 2013] L. Dembélé and A. Kumar, "Examples of abelian surfaces with everywhere good reduction", preprint, 2013. arXiv 1309.3821

[Dolgachev 1996] I. V. Dolgachev, "Mirror symmetry for lattice polarized K3 surfaces", J. Math. Sci. 81:3 (1996), 2599-2630. MR 97i:14024 Zbl 0890.14024

[Elkies 1998] N. D. Elkies, "Elliptic and modular curves over finite fields and related computational issues", pp. 21-76 in Computational perspectives on number theory (Chicago, IL), edited by D. A. Buell and J. T. Teitelbaum, AMS/IP Stud. Adv. Math. 7, Amer. Math. Soc., Providence, RI, 1998. MR 99a:11078 Zbl 0915.11036

[Elkies 2008] N. D. Elkies, "Shimura curve computations via $K 3$ surfaces of Néron-Severi rank at least 19", pp. 196-211 in Algorithmic number theory, edited by A. J. van der Poorten and A. Stein, Lecture Notes in Comput. Sci. 5011, Springer, Berlin, 2008. MR 2010e:11056 Zbl 1205.11069

[Elkies $\geq 2015]$ N. D. Elkies, "Elliptic curves of high rank over $\mathbb{Q}$ and $\mathbb{Q}(t)$ ”, In preparation.

[Friedman 1984] R. Friedman, "A new proof of the global Torelli theorem for K3 surfaces", Ann. of Math. (2) 120:2 (1984), 237-269. MR 86k:14028 Zbl 0559.14004

[van der Geer 1988] G. van der Geer, Hilbert modular surfaces, Ergebnisse der Mathematik und ihrer Grenzgebiete (3) [Results in Mathematics and Related Areas (3)] 16, Springer, Berlin, 1988. MR 89c:11073 Zbl 0634.14022 
[Gritsenko and Nikulin 1997] V. A. Gritsenko and V. V. Nikulin, "Siegel automorphic form corrections of some Lorentzian Kac-Moody Lie algebras", Amer. J. Math. 119:1 (1997), 181-224. MR 98g:11056 Zbl 0914.11020

[Gruenewald 2008] D. Gruenewald, Explicit Algorithms for Humbert Surfaces, Ph.D. thesis, University of Sydney, 2008, Available at http://echidna.maths.usyd.edu.au/ davidg/thesis.pdf.

[Hausmann 1982] W. Hausmann, "The fixed points of the symmetric Hilbert modular group of a real quadratic field with arbitrary discriminant”, Math. Ann. 260:1 (1982), 31-50. MR 84j:10036 Zbl 0467.10020

[Hirzebruch 1973] F. E. P. Hirzebruch, "Hilbert modular surfaces", Enseignement Math. (2) 19 (1973), 183-281. MR 52\#13856 Zbl 0285.14007

[Hirzebruch and van de Ven 1974] F. Hirzebruch and A. van de Ven, "Hilbert modular surfaces and the classification of algebraic surfaces", Invent. Math. 23 (1974), 1-29. MR 51 \#517 Zbl 0296.14020

[Hirzebruch and van der Geer 1981] F. Hirzebruch and G. van der Geer, Lectures on Hilbert modular surfaces, Séminaire de Mathématiques Supérieures 77, Presses de l'Université de Montréal, 1981. MR 83i:10037 Zbl 0483.14009

[Hirzebruch and Zagier 1977] F. Hirzebruch and D. Zagier, "Classification of Hilbert modular surfaces", pp. 43-77 in Complex analysis and algebraic geometry, edited by J. W. L. Baily and T. Shioda, Iwanami Shoten, Tokyo, 1977. MR 58 \#524 Zbl 0354.14011

[Hudson 1990] R. W. H. T. Hudson, Kummer's quartic surface, Cambridge University Press, 1990. MR 92e:14033 Zbl 0716.14025

[Igusa 1960] J.-i. Igusa, "Arithmetic variety of moduli for genus two", Ann. of Math. (2) 72 (1960), 612-649. MR 22 \#5637 Zbl 0122.39002

[Inose 1978] H. Inose, "Defining equations of singular $K 3$ surfaces and a notion of isogeny", pp. 495-502 in Proceedings of the International Symposium on Algebraic Geometry (Kyoto University, 1977), edited by M. Nagata, Kinokuniya Book Store, Tokyo, 1978. MR 81h:14021

[Khare and Wintenberger 2009a] C. Khare and J.-P. Wintenberger, "Serre's modularity conjecture, I", Invent. Math. 178:3 (2009), 485-504. MR 2010k:11087 Zbl 05636295

[Khare and Wintenberger 2009b] C. Khare and J.-P. Wintenberger, "Serre's modularity conjecture, II”, Invent. Math. 178:3 (2009), 505-586. MR 2010k:11088 Zbl 05636296

[Kulikov 1977] V. S. Kulikov, "Degenerations of $K 3$ surfaces and Enriques surfaces”, Izv. Akad. Nauk SSSR Ser. Mat. 41:5 (1977), 1008-1042, 1199. In Russian; translated in Math. USSR-Izv. 11:5 (1977), 957-989. MR 58 \#22087b Zbl 0367.14014

[Kumar 2008] A. Kumar, "K3 surfaces associated with curves of genus two", Int. Math. Res. Not. 2008:6 (2008), Art. ID rnm165, 26. MR 2009d:14044 Zbl 1145.14029

[Kumar 2014] A. Kumar, "Elliptic fibrations on a generic Jacobian Kummer surface”, J. Algebraic Geom. 23 (2014), 599-667.

[Kuwata and Shioda 2008] M. Kuwata and T. Shioda, "Elliptic parameters and defining equations for elliptic fibrations on a Kummer surface", pp. 177-215 in Algebraic geometry in East Asia (Hanoi, 2005), edited by K. Konno and V. Nguyen-Khac, Adv. Stud. Pure Math. 50, Math. Soc. Japan, Tokyo, 2008. MR 2009g:14039 Zbl 1139.14032

[Liu et al. 2005] Q. Liu, D. Lorenzini, and M. Raynaud, "On the Brauer group of a surface", Invent. Math. 159:3 (2005), 673-676. MR 2005k:14036 Zbl 1077.14023

[van Luijk 2007] R. van Luijk, "K3 surfaces with Picard number one and infinitely many rational points”, Algebra Number Theory 1:1 (2007), 1-15. MR 2008d:14058 Zbl 1123.14022 
[Mestre 1991] J.-F. Mestre, "Construction de courbes de genre 2 à partir de leurs modules", pp. 313-334 in Effective methods in algebraic geometry (Castiglioncello, 1990), edited by T. Mora and C. Traverso, Progr. Math. 94, Birkhäuser, Boston, 1991. MR 92g:14022 Zbl 0752.14027

[Milne 1975a] J. S. Milne, "On a conjecture of Artin and Tate", Ann. of Math. (2) 102:3 (1975), 517-533. MR 54 \#2659 Zbl 0343.14005

[Milne 1975b] J. S. Milne, “On the conjecture of Artin and Tate”, 1975, Available at http://jmilne.org/ math/articles/add/1975a.pdf. Notes on Ann. of Math. (2) 102:3 (1975), 517-533. Zbl 0343.14005

[Morrison 1984] D. R. Morrison, “On K3 surfaces with large Picard number”, Invent. Math. 75:1 (1984), 105-121. MR 85j:14071 Zbl 0509.14034

[Nikulin 1979a] V. V. Nikulin, "Finite groups of automorphisms of Kählerian K3 surfaces", Trudy Moskov. Mat. Obshch. 38 (1979), 75-137. In Russian. MR 81e:32033 Zbl 0433.14024

[Nikulin 1979b] V. V. Nikulin, "Integer symmetric bilinear forms and some of their geometric applications", Izv. Akad. Nauk SSSR Ser. Mat. 43:1 (1979), 111-177, 238. In Russian; translated in Math. USSR-Izv. 14:1 (1980), 103-167. MR 80j:10031 Zbl 0408.10011

[Oda 1982] T. Oda, Periods of Hilbert modular surfaces, Progress in Mathematics 19, Birkhäuser, Boston, 1982. MR 83k:10057 Zbl 0489.14014

[Persson and Pinkham 1981] U. Persson and H. Pinkham, "Degeneration of surfaces with trivial canonical bundle", Ann. of Math. (2) 113:1 (1981), 45-66. MR 82f:14030 Zbl 0426.14015

[Piatetski-Shapiro and Shafarevich 1971] I. Piatetski-Shapiro and I. R. Shafarevich, "Torelli's theorem for algebraic surfaces of type K3", Izv. Akad. Nauk SSSR Ser. Mat. 35 (1971), 530-572. In Russian; translated in Math. USSR-Izv. 5:3 (1971), 547-588. MR 44 \#1666 Zbl 0219.14021

[Rapoport 1978] M. Rapoport, "Compactifications de l'espace de modules de Hilbert-Blumenthal", Compositio Math. 36:3 (1978), 255-335. MR 80j:14009 Zbl 0386.14006

[Ribet 2004] K. A. Ribet, "Abelian varieties over Q and modular forms", pp. 241-261 in Modular curves and abelian varieties, edited by J. Cremona et al., Progr. Math. 224, Birkhäuser, Basel, 2004. MR 2005k:11120 Zbl 1092.11029

[Runge 1999] B. Runge, "Endomorphism rings of abelian surfaces and projective models of their moduli spaces", Tohoku Math. J. (2) 51:3 (1999), 283-303. MR 2000g:14056 Zbl 0972.14017

[Shioda 1972] T. Shioda, "On elliptic modular surfaces", J. Math. Soc. Japan 24 (1972), 20-59. MR 55 \#2927 Zbl 0226.14013

[Shioda 1990] T. Shioda, "On the Mordell-Weil lattices", Comment. Math. Univ. St. Paul. 39:2 (1990), 211-240. MR 91m:14056 Zbl 0725.14017

[Shioda 2006] T. Shioda, "Kummer sandwich theorem of certain elliptic $K 3$ surfaces", Proc. Japan Acad. Ser. A Math. Sci. 82:8 (2006), 137-140. MR 2008b:14064 Zbl 1112.14044

[Tate 1966a] J. Tate, "Endomorphisms of abelian varieties over finite fields", Invent. Math. 2 (1966), 134-144. MR 34 \#5829 Zbl 0147.20303

[Tate 1966b] J. Tate, "On the conjectures of Birch and Swinnerton-Dyer and a geometric analog", in Séminaire Bourbaki 1965/1966 (Exposé 306), W. A. Benjamin, Amsterdam, 1966. Reprinted as pp. 415-440 in Séminaire Bourbaki 9, Soc. Math. France, Paris, 1995. MR 1610977 Zbl 0199.55604

[Tate 1975] J. Tate, "Algorithm for determining the type of a singular fiber in an elliptic pencil", pp. 33-52 in Modular functions of one variable, IV, edited by B. J. Birch and W. Kuyk, Lecture Notes in Math. 476, Springer, Berlin, 1975. MR 52 \#13850 Zbl 1214.14020

[Wilson 1998] J. Wilson, Curves of genus 2 with real multiplication by a square root of 5, Ph.D. thesis, Oxford University, 1998, Available at http://eprints.maths.ox.ac.uk/32/1/wilson.pdf. 
[Wilson 2000] J. Wilson, "Explicit moduli for curves of genus 2 with real multiplication by $\mathbf{Q}(\sqrt{5})$ ", Acta Arith. 93:2 (2000), 121-138. MR 2001f:11099 Zbl 0966.11027

Communicated by Ravi Vakil

Received 2013-01-22 Revised 2013-08-26 Accepted 2013-10-28

elkies@math.harvard.edu Department of Mathematics, Harvard University, Cambridge, MA 02138, United States

abhinav@math.mit.edu

Department of Mathematics, Massachusetts Institute of Technology, Cambridge, MA 02139, United States 


\section{Algebra \& Number Theory}

msp.org/ant

\section{EDITORS}

MANAGING EDITOR

Bjorn Poonen

Massachusetts Institute of Technology

Cambridge, USA

\author{
EDITORIAL BOARD CHAIR \\ David Eisenbud \\ University of California \\ Berkeley, USA
}

BOARD OF EDITORS

Georgia Benkart

Dave Benson

Richard E. Borcherds

John H. Coates

J-L. Colliot-Thélène

Brian D. Conrad

Hélène Esnault

Hubert Flenner

Edward Frenkel

Andrew Granville

Joseph Gubeladze

Roger Heath-Brown

Craig Huneke

János Kollár

Yuri Manin

Barry Mazur

Philippe Michel

Susan Montgomery
University of Wisconsin, Madison, USA

University of Aberdeen, Scotland

University of California, Berkeley, USA

University of Cambridge, UK

CNRS, Université Paris-Sud, France

University of Michigan, USA

Freie Universität Berlin, Germany

Ruhr-Universität, Germany

University of California, Berkeley, USA

Université de Montréal, Canada

San Francisco State University, USA

Oxford University, UK

University of Virginia, USA

Princeton University, USA

Northwestern University, USA

Harvard University, USA

École Polytechnique Fédérale de Lausanne

University of Southern California, USA
Shigefumi Mori

Raman Parimala

Jonathan Pila

Anand Pillay

Victor Reiner

Peter Sarnak

Joseph H. Silverman

Michael Singer

Vasudevan Srinivas

J. Toby Stafford

Bernd Sturmfels

Richard Taylor

Ravi Vakil

Michel van den Bergh

Marie-France Vignéras

Kei-Ichi Watanabe

Efim Zelmanov

Shou-Wu Zhang
RIMS, Kyoto University, Japan

Emory University, USA

University of Oxford, UK

University of Notre Dame, USA

University of Minnesota, USA

Princeton University, USA

Brown University, USA

North Carolina State University, USA

Tata Inst. of Fund. Research, India

University of Michigan, USA

University of California, Berkeley, USA

Harvard University, USA

Stanford University, USA

Hasselt University, Belgium

Université Paris VII, France

Nihon University, Japan

University of California, San Diego, USA

Princeton University, USA

\section{PRODUCTION}

production@msp.org

Silvio Levy, Scientific Editor

See inside back cover or msp.org/ant for submission instructions.

The subscription price for 2014 is US \$225/year for the electronic version, and $\$ 400 /$ year ( $\$ 55$, if shipping outside the US) for print and electronic. Subscriptions, requests for back issues and changes of subscribers address should be sent to MSP.

Algebra \& Number Theory (ISSN 1944-7833 electronic, 1937-0652 printed) at Mathematical Sciences Publishers, 798 Evans Hall \#3840, c/o University of California, Berkeley, CA 94720-3840 is published continuously online. Periodical rate postage paid at Berkeley, CA 94704, and additional mailing offices.

ANT peer review and production are managed by EditFLOW ${ }^{\circledR}$ from MSP.

\section{PUBLISHED BY}

mathematical sciences publishers

nonprofit scientific publishing

http://msp.org/

(C) 2014 Mathematical Sciences Publishers 


\section{Algebra \& Number Theory}

Volume $8 \quad$ No. $10 \quad 2014$

K3 surfaces and equations for Hilbert modular surfaces

NOAM ELKIES and ABHINAV KUMAR

Intermediate co- $t$-structures, two-term silting objects, $\tau$-tilting modules, and torsion classes

OSAMU IyAma, Peter JøRgensen and Dong YANG

A $p$-adic Eisenstein measure for vector-weight automorphic forms

ELLEN EISCHEN

Explicit points on the Legendre curve III

DOUGLAS ULMER

Explicit Gross-Zagier and Waldspurger formulae

Li CAI, JiE SHU and Ye Tian 